Verena Kuglstatter

Der Gebrauch der

Jugendgewaltprävention

Subjektivierungsformen eines

Problemdiskurses

[transcript] Gesellschaft der Unterschiede 
Verena Kuglstatter

Der Gebrauch der Jugendgewaltprävention

Gesellschaft der Unterschiede | Band 43 
Verena Kuglstatter, geb. 1983 , arbeitet als wissenschaftliche Mitarbeiterin an der Hamburger Fern-Hochschule im Fachbereich Gesundheit und Pflege und forscht zu Formen sozialen Engagements Jugendlicher, peerspezifischen Sozialisationsprozessen und Jugendgewaltprävention. 
Verena Kuglstatter

\section{Der Gebrauch der Jugendgewaltprävention}

Subjektivierungsformen eines Problemdiskurses

[transcript] 
Die vorliegende Arbeit wurde von der Philosophischen Fakultät der Universität Zürich im Frühjahrssemester 2016 auf Antrag der Promotionskommission Prof. Dr. Jan Skrobanek (hauptverantwortliche Betreuungsperson) und Prof. Dr. Peter Rieker als Dissertation angenommen.

Publiziert mit Unterstützung des Schweizerischen Nationalfonds zur Förderung der wissenschaftlichen Forschung.

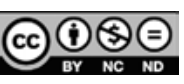

Dieses Werk ist lizenziert unter der

Creative Commons Attribution 3.0 (BY-NC-ND).

Dieses Werk ist lizenziert unter der Creative Commons Attribution-Non Commercial-NoDerivs 3.0 DE Lizenz (BY-NC-ND). Diese Lizenz erlaubt die private Nutzung, gestattet aber keine Bearbeitung und keine kommerzielle Nutzung. Weitere Informationen finden Sie unter

https://creativecommons.org/licenses/by-nc-nd/3.o/de/.

\section{Bibliografische Information der Deutschen Nationalbibliothek}

Die Deutsche Nationalbibliothek verzeichnet diese Publikation in der Deutschen Nationalbibliografie; detaillierte bibliografische Daten sind im Internet über http://dnb.d-nb.de abrufbar.

\section{() 2017 transcript Verlag, Bielefeld}

Die Verwertung der Texte und Bilder ist ohne Zustimmung des Verlages urheberrechtswidrig und strafbar. Das gilt auch für Vervielfältigungen, Übersetzungen, Mikroverfilmungen und für die Verarbeitung mit elektronischen Systemen.

Umschlagkonzept: Kordula Röckenhaus, Bielefeld

Korrektorat: Katrin Herbon, Bonn

Satz: Mark-Sebastian Schneider, Bielefeld

Druck: Majuskel Medienproduktion GmbH, Wetzlar

Print-ISBN 978-3-8376-3898-I

PDF-ISBN 978-3-8394-3898-5

Gedruckt auf alterungsbeständigem Papier mit chlorfrei gebleichtem Zellstoff. Besuchen Sie uns im Internet: http://www.transcript-verlag.de

Bitte fordern Sie unser Gesamtverzeichnis und andere Broschüren an unter: info@transcript-verlag.de 


\section{Inhalt}

\section{Einleitung $\mid 7$}

2. Theoretische Reflexion des Forschungsgegenstands $\mid 13$

2.1 Diskurstheoretische Perspektive auf Jugendgewaltprävention $\mid 13$

2.2 Über das Verhältnis von Diskurs und diskursiven Praktiken | 25

2.3 Über das Verhältnis von Subjekt, Macht und Wissen 29

2.4 Sprechen über Gewalt: Über das Verhältnis von Sprechen und Gewalt | 34

\section{Methodische Herangehensweise $\mid 37$}

3.1 Zur Verknüpfung von Diskursanalyse und Dokumentarischer Methode $\mid 37$

3.2 Feldzugang und Datenmaterial $\mid 47$

4. Datenauswertung $\mid 57$

4.1 (Jugendgewalt-)Prävention aus der Perspektive der Fachpersonen $\mid 57$

4.1.1 Interpretation der Einstiegspassagen: Doing Expertise $\mid 59$

4.1.2 Prävention zwischen Gesellschaftskritik

und kriminalpolitischer Programmatik | 102

4.1.3 Zur Normalisierung von Jugendgewalt $\mid 138$

4.1.4 Zusammenfassung $\mid 152$

4.2 Jugendgewaltprävention auf Ebene des Programms $\mid 157$

4.2.1 Zur Konstruktion von Kollektivität | 159

4.2.2 Zur Konstruktion von »Jugend «| 166

4.2.3 Legitimationsweisen $\mid 172$

4.2.4 Zusammenfassung 177

4.3 Zusammenfassung und Integration der Ergebnisse 179

4.4 Methodische Reflexion 187

\section{Theoretische Diskussion der Ergebnisse 191}

5.1 Responsibilisierung und die Individualisierung von Risiko | 191

5.2 Verantwortung als Subjektivierungsform $\mid 200$

5.3 Thesen $\mid 207$ 
6. Theoretische Anschlussdiskussion | 211

6.1 Jugendsoziologische Anschlüsse | 211

6.2 Gewalttheoretische Anschlüsse | 214

7. Zum Gebrauch von Prävention | 221

7.1 Paradoxien im Kontext von Jugendgewaltprävention | 221

7.2 Kritik einer Gesellschaft der Bestandssicherung | 222

Literatur $\mid 225$ 


\section{Einleitung}

Die hier vorliegende Arbeit beschäftigt sich mit Jugendgewaltprävention. Nein! Es geht weder um die Frage »Wie gelingt Jugendgewaltprävention?« noch um die Frage »Welche Projekte sind wirksam?«. Die Konfrontation der Forscherin mit diesen Fragen im Laufe des Forschungsprozesses, sofern sie auch nur Andeutungen über das von ihr beforschte Thema gemacht hat, weist darauf hin, dass dieses von kriminologischen Problemstellungen geradezu okkupiert ist. Die aus kriminologischer Perspektive für relevant gesetzte Fragen bezüglich der Güte und Wirksamkeit von Jugendgewaltprävention und, damit verbunden, der Ursachen von Jugendgewalt weichen hier grundlegenden Perspektiven von Fachpersonen im Kontext von Jugendgewaltprävention: ${ }^{1}$ Welche Relevanz sprechen die Fachpersonen Jugendgewaltprävention hinsichtlich ihrer jeweiligen Handlungskontexte $\mathrm{zu}$, wie begründen sie diese und welche Problematisierungsweisen stehen damit in Zusammenhang? Dabei geht diese

1 | Die soziologische Erforschung von Gewalt steht im Ruf, erheblich von kriminologisch für relevant gesetzten Fragen, insbesondere nach den Ursachen von Gewalt, geprägt zu sein, und als "Soziologie der Ursachen von Gewalt" (Stehr 2009: 118) in der Kritik (vgl. Cremer-Schäfer 2016: 17f. zur Kritik der "Ätiologie" als "ein in allen bisherigen kapitalistischen Produktionsweisen und Politikformen herrschendes Wissenschaftsparadigma«; Groenemeyer 2012: 36 zur Problematisierung sozialer Probleme als nicht hinterfragbare Voraussetzung soziologischer Forschung; Koloma Beck/Schlichte 2014: 106f. über die einseitige Problematisierung von Gewalt in soziologischer Theoriebildung und Forschung, mit der ein Mangel an einer sozialtheoretischen Auseinandersetzung mit Gewalt einhergeht; Scherr 2010: 47f. zur Überlagerung sozialwissenschaftlicher Jugendforschung durch politische, kriminologische, mediale und pädagogische Diskurse; Trotha 1997: 20f.). Genuin soziologische Fragestellungen, die sich mit dem Gewaltbegriff und mit der Entstehung von Gewalt (v.a. auf phänomenologischer Ebene) auseinandersetzen oder, wie hier, gesellschaftliche Problematisierungsweisen von Gewalt in einem bestimmten Kontext kritisch beleuchten und dabei das Verhältnis von Individuum und Gesellschaft fokussieren, stehen neben kriminologischen Fragestellungen, die primär Ursachen von Gewalt betreffen. 
Arbeit davon aus, dass sowohl diesbezügliche Konstruktionen von Devianz als auch damit verbundene Professionsverständnisse, Präventionskonzepte und deren Legitimierung aus der Perspektive von »Experten« unterschiedlicher Fachbereiche im Kontext von Jugendgewaltprävention auf »Präventionsdiskurse« verweisen. Sie stellt damit die Frage ins Zentrum, inwiefern sich Fachpersonen im Kontext von Jugendgewaltprävention hinsichtlich ihrer Problematisierungsweisen, ihres Präventionsverständnisses und damit einhergehenden Handlungs- und Begründungsweisen an »Präventionsdiskursen« orientieren. Diskurstheoretisch gesprochen fokussiert die Arbeit »über den Gebrauch von Prävention« Subjektivierungsformen im Kontext von Jugendgewaltprävention. Diese Orientierungsweisen, so die Annahme, stellen grundsätzliche Weichen hinsichtlich der Bearbeitung »sozialer Probleme«, insbesondere im Kontext der Jugendarbeit, und formen den Gestaltungsspielraum sowohl der Fachpersonen als auch der Jugendlichen in maßgeblicher Weise.

Motiviert ist diese Fragestellung von der Beobachtung, dass »Präventionsdiskurse« primär kriminologisch geprägt sind und um Fragen von Risiken, Wirksamkeit und Evidenz kreisen (vgl. Reder/Ziegler 2011: 367) sowie im Kontext einer aktivierenden Sozialpolitik gelesen werden können (vgl. Bröckling 2009: 214; Huber 2014: 42; Lindenberg/Ziegler 2005: 619; Luedtke/Wiezorek 2016: 7f.; Ziegler 2001: 194). Wie schließen Fachpersonen unterschiedlicher Bereiche im Sprechen über Jugendgewaltprävention an kriminalpolitische Programmatik an, wenn davon auszugehen ist, dass sie weniger Risiken als konkrete Individuen in spezifischen Kontexten adressieren (vgl. Lindenberg/ Ziegler 2005: 614; Reder/Ziegler 2011: 369 ) und dabei jeweils spezifische Ziele verfolgen und jeweils spezifischen Restriktionen unterworfen sind, innerhalb derer weniger Fragen der Wirksamkeit im Sinne einer messbaren Größe als vielmehr erfahrungsbasierte Dimensionen der Wirksamkeit relevant werden?2

2 | Die hier vorliegende Arbeit orientiert sich u.a. an Texten und empirischen Studien zu "kommunaler Gewalt- oder Kriminalprävention“. Der Begriff der kommunalen Gewaltprävention ist für den im Kontext der Schweiz angesiedelten Diskurs über Jugendgewaltprävention aus verschiedenen Gründen, die noch genauer erläutert werden, unpassend. Auf zwei Gründe soll hier kurz eingegangen werden: Erstens ist der spezifische Diskurs über Jugendgewaltprävention nicht gleichzusetzen mit (allgemeiner) kommunaler Gewalt- oder Kriminalprävention, sondern damit ebenso verwoben wie mit "Jugendpolitiken", über welche "die Lage, der Handlungsrahmen und die Individualisierungs- und Autonomiemöglichkeiten von Jugendlichen beeinflusst werden" (Luedtke/Wiezorek 2016: 7). Zweitens stehen im Fokus der hier durchgeführten Analyse Programminhalte eines spezifischen Programms in der Schweiz, des "Nationalen Präventionsprogramms Jugend und Gewalt", das Inhalte und Ziele betreffend Parallelen zu "kommunaler Gewalt- oder Kriminalprävention ", aber auch Unterschiede dazu aufweist. 
Bernd Dollinger und Henning Schmidt-Semisch sprechen im Kontext allgemeiner Kriminalprävention von einem Zwang der Kooperation zwischen Kriminologie und Sozialpädagogik, die »einen kriminal- und sozialpolitischen Auftrag bildet, zu dem sich die Akteure in diesen Feldern, in welcher konkreten Form auch immer, zu verhalten haben « (Dollinger/Schmidt-Semisch 2011: 14). Sie plädieren für einen Dialog, der Raum für unterschiedliche Ansätze ermöglicht (vgl. ebd.: 17). Insofern beleuchtet die vorliegende Arbeit einen Gegenstand, der im Zusammenhang aktueller Auseinandersetzungen um das Verhältnis von Sozialer Arbeit und Politik relevant, jedoch empirisch wenig thematisiert bzw. erforscht ist (vgl. Schönig 2013: 33f.). Insbesondere fehlen diesbezüglich auch kritische empirische Auseinandersetzungen im Kontext von Jugendarbeit und Jugendhilfe, in dem Prävention in den letzten Jahrzehnten zu einer dominanten Richtlinie geworden ist (Widersprüche 2016: 4). Eine diskurstheoretische Annäherung an Fragen des Verhältnisses zwischen Kri-

Um eine Spezifizierung des Gegenstands vorzunehmen, wird der Begriff der kriminalpolitischen Programmatik gewählt, womit die Orientierungsweisen von Fachpersonen im Kontext von Jugendgewaltprävention an so etwas wie einem "Präventionsdiskurs" benennbar werden. Die Bezeichnung "Präventionsdiskurs" ist ebenfalls unpassend, da dieser in komplexe Diskurszusammenhänge eingebettet ist von denen er nicht zu trennen ist. Das "Nationale Präventionsprogramm Jugend und Gewalt" als kriminalpolitisches Programm zu bezeichnen, ist hinsichtlich des Vergleichs mit der "kommunalen Kriminalprävention “ jedoch durchaus plausibel, insofern als "das Phänomen 'kommunale Kriminalprävention، als kriminalpolitische Bewegung beschrieben werden kann“ (Berner/Groenemeyer 2003: 85), das sozialpolitischen Interessen gegenübersteht. Da sich die befragten Fachpersonen jedoch nicht oder kaum explizit zu diesem Programm positionieren und sich vielmehr auf komplexe Diskurszusammenhänge beziehen, sieht die Autorin dieser Arbeit den Begriff der "kriminalpolitischen Programmatik" als sinnvoll an. Dieser Begriff irritiert und lenkt damit den Blick gleichzeitig auf einen relevanten Sachverhalt: Das "Nationale Programm Jugend und Gewalt" steht zwar in einem komplexen Diskurszusammenhang, ist aber, rein formal, auf sozialpolitischer Ebene angesiedelt (Bundesamt für Sozialversicherung als Teil des Eidgenössischen Departements des Inneren Altersvorsorge und Gesundheit). Nichtsdestotrotz thematisiert es primär kriminalpolitische Fragestellungen und erhebt die Kriminologie als ersten und obersten Bezugspunkt im Kontext von Jugendgewaltprävention. Der Begriff der Programmatik beschreibt eine Art von Rationalität, die wirkmächtig ist: „Prävention ist hierbei als politische Programmatik zu verstehen, die auf dem Prinzip der Vorbeugung und damit des Vorausgreifens beruht." (Lindenau/Münkler 2012: 64) Der Begriff verbindet also die Beobachtung, dass das "Nationale Präventionsprogramm Jugend und Gewalt" sozialpolitische Fragestellungen kriminalpolitisch und mit einer der Prävention inhärenten Logik bearbeitet. Somit stellt er in der hier vorliegenden Arbeit einen kritischen und gleichzeitig analytischen Bezugspunkt dar. 
minologie und Sozialer Arbeit bzw. Sozialpädagogik ist insofern sinnvoll, als die Orientierungen von Fachpersonen im Kontext von Jugendgewaltprävention an kriminalpolitischer Programmatik nicht nur als Prozesse der Adressierung, sondern auch der Subjektivierung anzusehen sind. Die Verwendung des Subjektbegriffs als analytische Kategorie ermöglicht damit nicht nur Einblicke in fachspezifische Herausforderungen Sozialer Arbeit, sondern darüber hinaus auch Perspektiven auf ungleichheitsrelevante Verhältnisse zwischen Individuum und Gesellschaft. Die Fachpersonen kommen also als Subjekte in den Blick. Schließlich handelt es sich beim »Diskurs« über Jugendgewaltprävention nicht um einen Diskurs, der lediglich Fachpersonen in die Verantwortung nimmt, sondern der auch über eine bestimmte Bevölkerungsgruppe, in diesem Fall Jugendliche, geführt wird. Jugendliche sind demnach als Objekte des »Diskurses« über Jugendgewaltprävention anzusehen. Im Anschluss an eine Soziologie sozialer Probleme werden also Konstruktionsweisen von Jugend als soziale Gruppe über Problemdiskurse fokussiert und demnach »die Frage nach den Prozessen und Bedingungen der öffentlichen, politischen und wissenschaftlichen Thematisierung und der Problematisierung von Jugend und Jugendproblemen in der Gesellschaft zu einem zentralen Forschungsgegenstand gemacht« (Groenemeyer 2014: 51; vgl. auch Anhorn 2002: 48; Griese 2014: 18; Luedtke/Wiezorek 2016: 7f.; Scherr 2014: 35). Insofern geht die Frage nach Prozessen der Subjektivierung mit der Frage nach Prozessen der Objektivierung sozialer Gruppen einher. Die Auseinandersetzung mit Jugendgewaltprävention erfolgt hier primär machttheoretisch im Anschluss an Michel Foucault. Mit den diskurstheoretisch zentralen Begriffen der Macht, des Subjekts und des Wissens werden mit Jugendgewaltprävention verbundene Aspekte der Regulierung von Bevölkerungsgruppen thematisiert (vgl. Foucault 2004a). Foucault folgend wird damit nicht auf ein negatives, sondern auf ein positives Konzept von Macht rekurriert, insofern Macht nicht nur bemächtigt, sondern gleichzeitig auch entmächtigt (vgl. Han 2010: 44f.). An die verschiedenen einleitend angerissenen Beobachtungen und theoretischen Ansätze anschließend wird die zentrale Forschungsfrage festgehalten: In welcher Weise orientieren sich Fachpersonen im Kontext von Jugendgewaltprävention an kriminalpolitischer Programmatik und welche Konsequenzen folgen daraus für Soziale Arbeit und Jugendliche? Methodisch nähert sich die Arbeit der Forschungsfrage an, indem sie primär zwei Datenquellen kontrastierend untersucht: Die Untersuchung offener leitfadengestützter Interviews mit Fachpersonen aus unterschiedlichen Bereichen der Jugendgewaltprävention mittels der dokumentarischen Methode gibt Aufschluss über die jeweiligen Deutungs-, Konstruktions-, Handlungs- und Legitimationsweisen bezüglich Jugendgewaltprävention. Diese wird (anschließend) in Bezug gesetzt zur Untersuchung von Dokumenten, insbesondere eines Transkripts und zweier Protokolle, die sich auf die im Rahmen des »Nationalen Präventionsprogramms Jugend und Ge- 
walt« veranstalteten Konferenzen beziehen. Dabei handelt es sich um ein auf fünf Jahre angelegtes Programm, das vom Bundesrat in Auftrag gegeben worden ist; ${ }^{3}$ in dessen Auftrag ist es im Jahr 2015 mit dem Ziel »die von Seiten des Programms zur Verfügung gestellten Unterstützungsinstrumente unter den Gesichtspunkten Relevanz, Nutzung, Nützlichkeit, Wirkung und Nachhaltigkeit zu beurteilen « (Féraud/Huegli 2015) evaluiert und schließlich mit der Begründung beendet worden, dass »die Gewaltprävention eine Aufgabe von Kantonen, Städten und Gemeinden ist« (Bericht des Bunderates 2015: 81). Das »Nationale Präventionsprogramm Jugend und Gewalt«, das zum vordersten Ziel hat, Jugendgewaltprävention u.a. durch flächendeckende Implementierung sog. »evidenzbasierter Projekte s schweizweit zu standardisieren, ruft unterschiedlichste Fachbereiche in ihrer Verantwortung an. Vernetzung, Austausch und die Schaffung einer gemeinsamen Wissensbasis sollen zur Realisierung der gemeinsamen Ziele beitragen. Die Metapher, dass alle im selben Boot sitzen, ist dabei zentral und verweist auf die Bedeutung utopischer und dystopischer Szenarien für die Legitimation kriminalpräventiver Strategien (vgl. Liell 2002: 6; Schreiber 2011: 145f.). Insofern der Gegenstand der hier vorliegenden Arbeit die Analyse der »Denk- und Sichtweisen, die Programme vermitteln, und die Frage ist, wie diese Sichtweisen sich in Technologien des Regierens, also in systematischen Praktiken der Menschenführung realisieren« (Kessl/Krasmann 2005: 232), ist die Arbeit auch als Programmanalyse zu verstehen. Dabei beschränkt sie sich nicht auf die Untersuchung von mit dem Programm in Zusammenhang stehenden Dokumenten, sondern verknüpft diese mit der Untersuchung von Interviewdaten. Dies stellt eine methodische Herausforderung dar, der hier besondere Aufmerksamkeit gewidmet wird. Die Orientierung der im Kontext von Jugendgewaltprävention tätigen Fachpersonen an dieser Programmatik ist nicht nur hinsichtlich des Verständnisses von Jugendgewaltprävention relevant, sondern bereits hinsichtlich der je spezifischen Problematisierungsweisen, die Jugendgewaltprävention vorausgehen, insofern grundlegende (Be-)Deutungen von Jugendgewalt in Problemdiskurse eingebettet sind (vgl. Anhorn 2002: 54; Griese 2012: 697; Scherr 2014: 36). In diesem Zusammenhang wird der Frage nachgegangen, auf welchen Verständ-

3 | "Für den Aufbau eines Gesamtschweizerischen Programms zur Prävention und Bekämpfung von Jugendgewalt lädt der Bundesrat die Vertreterinnen und Vertreter der Kantone, Städte und Gemeinden ein, gemeinsam mit dem Bund Struktur, Inhalt, Funktionsweise und Finanzierung des gemeinsamen Programms konzeptionell auszuarbeiten. Das EDI (BSV) wird beauftragt, die Koordination dieser Arbeiten sicherzustellen und zu diesem Zweck eine breit abgestützte Arbeitsgruppe einzusetzen. Die Konzeptionsphase soll bis Anfang 2010 dauern; der Bundesrat wird dann aufgrund der Vorlage der Detailkonzeption über die finanzielle Beteiligung und den personellen Mittelaufwand des Bundes entscheiden." (Bericht des Bunderates 2009: 88) 
nissen von Jugendgewalt bzw. damit zusammenhängenden Problematisierungsweisen Jugendgewaltprävention basiert, oder anders formuliert, wie Jugendgewaltprävention legitimiert wird. Eine gegenstandstheoretische Diskussion folgt schließlich hinsichtlich Sozialer Arbeit und eine theoretische Anschlussdiskussion hinsichtlich Jugend und Gewalt aus soziologischer Perspektive. Gewalt wird als Sprechen über Jugendgewalt analysiert und gerät somit als diskursives Konstrukt in den Blick, das die Wahrnehmung von Jugendgewalt als Phänomen über die Konstruktion von Wahrnehmbarkeiten beeinflusst (vgl. Krasmann 1997: 96; Krasmann/Scheerer 1997: 8f.; Kreissl 1997: 184ff.; Schmidt 2015). Diese Perspektive auf Jugendgewalt führt abschließend zu der Frage, wie das Thema Jugend und Gewalt aus soziologischer Perspektive beforscht werden kann. Können diese Konstrukte analytisch dienlich sein und/oder als Gegenstände in den Blick geraten? 


\section{Theoretische Reflexion des Forschungsgegenstands}

\subsection{Diskurstheoretische Perspektive auf JUGENDGEWALTPRÄVENTION}

Die vorliegende Arbeit nähert sich empirisch dem Verhältnis von sozialer Praxis und kriminalpolitischer Programmatik über einen diskurstheoretischen Zugang. Dabei werden auch gewalttheoretische Ansätze, jugendsoziologische Perspektiven sowie Theorien Sozialer Arbeit befruchtet. Im Folgenden werden die Relevanzen einer diskurstheoretischen Perspektive auf Jugendgewaltprävention und die damit verbundenen methodologischen und methodischen Schlussfolgerungen erörtert. Damit wird die grundlegende Perspektive auf Jugendgewaltprävention eröffnet, grundlegende Begriffe eingeführt, eine Gegenstandsbestimmung geschaffen und die Forschungsfrage geschärft. Im Gegensatz zu kriminologischen Ansätzen, die den Gegenstand der Jugendgewalt gewissermaßen okkupieren, setzt die vorliegende Arbeit diesen Gegenstand nicht voraus, sondern geht davon aus, dass soziale Prozesse, hier in politischen und pädagogischen Bereichen, ihren Gegenstand und dessen Problematisierung (mit-)erzeugen. So ist eine der zentralen Thesen, dass Fachpersonen im Kontext von Jugendgewaltprävention Jugendgewalt durch die Orientierung an kriminalpolitischer Programmatik als problematischen und bearbeitbaren Gegenstand rekonstruieren. In welcher Weise sie das tun und welche Konsequenzen dies für die Adressatinnen und Adressaten bzw. Subjekte kriminalpolitischer Programmatik hat, ist Gegenstand der Analyse. Untersuchungsgegenstand sind in diesem Sinne »diskursive Praktiken« im Kontext von Jugendgewaltprävention, wobei sich diese Arbeit auf Foucaults »Archäologie des Wissens« (1994a), seine Studien über Sexualität (1979; 1986), seine Vorlesungen zur Gouvernementalität (2004a, b), die »sich mit der Entstehung eines politischen Wissens, das den Begriff der Bevölkerung und die Mechanismen zur Lenkung der Bevölkerung in den Mittelpunkt stellte, befaßten« (Foucault 2004a: 520), sowie auf sich daran anknüpfende theoretische und methodologische Ansätze wie diskurstheoretische Ansätze, Programmanaly- 
sen, »kritische Kriminologie« und die »Soziologie sozialer Probleme« bezieht. Mit der Fokussierung auf die Untersuchung »diskursiver Praktiken « werden aktuelle, primär theoretische Überlegungen der Diskursforschung zu Konstruktionsprozessen an Schnittstellen von »Diskursen « und »Praktiken « aufgegriffen (vgl. Bührmann 1997, 1998, 1999; Bührmann/Schneider 2008, 2010; Bröckling/Krasmann 2010; Kögler 2007; Luutz 1994; Marktschukat 2001; Ott/ Wrana 2010; Reckwitz 2008a, b), was Einblicke in Prozesse der (Re-)Konstruktion von Deutungsmustern bezüglich »Jugendgewalt« im Kontext von Jugendgewaltprävention gewährt, insofern als Deutungsmuster Wissen über einen Gegenstand ermöglichen, an das soziales Handeln anschließt (vgl. Althoff 2002a: 72, b: 78; Keller 2001: 123, 2011: 108; Reckwitz 2000: 298). Dabei gerät Prävention als »Regierungstechnologie« (Foucault 2004b: 442) in den Blick, die Individuen zuschreibbar und damit regierbar macht (vgl. Bührmann/ Schneider 2008: 113f.). Kinder und Jugendliche sind als die Zielscheibe von Prävention schlechthin anzusehen:

"Nicht alle Bürgerinnen und Bürger dieser Gesellschaft sind gleichermaßen und gleicherweise 'Gegenstand von Prävention. Hauptsächlich richten sich die diversen personenbezogenen Programme an Kinder und Jugendliche, an die Heranwachsenden, die in zwanzig/dreißig Jahren in der Mitte ihres Lebens diese Gesellschaft übernehmen bzw. ,in sie hineingewachsen sein sollen`." (Kappeler 2016: 58)

Über den Gebrauch von Jugendgewaltprävention, so die zentrale These, wird Jugendgewalt als problematischer Gegenstand verfestigt. Jugendliche werden damit einer per se problematischen Bevölkerungsgruppe zugerechnet und zu Adressatinnen bzw. Adressaten verschiedener Organisationen und Institutionen, insbesondere sozialpädagogischer Kontexte. Bei der hier vorliegenden Arbeit handelt es sich also um eine Diskursanalyse, insoweit ihr Interesse der Rekonstruktion von Deutungsmustern im Sinne einer Orientierung von Fachpersonen im Kontext von Jugendgewaltprävention an kriminalpolitischer Programmatik gilt. Die methodische Differenzierung zwischen Interviews und die Programmatik betreffenden Dokumenten ist dabei gewissermaßen künstlich, da theoretisch keine Unterscheidung zwischen »Äußerungen« im Rahmen von Interviews und im Rahmen eines kriminalpolitischen Programms getroffen wird. Gegenstand der Analyse sind Beziehungen von »Äußerungen «. ${ }^{1}$ Fabian Kessl spricht in diesem Zusammenhang von »diskursiven Praktiken«:

1 | Auf methodische Probleme bezüglich der Integration interpretativer Verfahren in diskurstheoretische Herangehensweisen wird in den Kapiteln 3.1 und 4.1 ausführlich eingegangen. 
"Untersuchungsgegenstand diskursanalytischer Vorgehensweisen sind somit hegemoniale diskursive Praktiken (Äußerungssysteme), das zu einem historisch-spezifischen Zeitpunkt Sagbare - und damit auch Sichtbare (Hegemoniale). [...] Diskursanalytische Vorgehensweisen sind weder auf Programmanalysen zu reduzieren, das heißt auf Materialrekonstruktionen programmatischer (Politik)Papiere, wenn diese als direkte Praxisregulation verstanden werden, noch auf Praxisanalysen, das heißt auf eine Rekonstruktion sozialer Praktiken, die fälschlicherweise als reale menschliche Praxis ,jenseits des Diskursiven gedeutet werden." (Kessl 2010: 352)

Aus diskurstheoretischer Perspektive sind Interviews als »diskursive Praktiken« zu verstehen, insofern sie im »Sprechen über« unvermeidlich auf »hegemoniales« Wissen verweisen. Gegenstand der hier vorliegenden Arbeit sind also »Äußerungen« in kriminalpolitischen und sozialpädagogischen Kontexten, wobei die Frage, inwiefern Fachpersonen im Sprechen über ihren je spezifischen Kontext auf diskursiv erzeugte Wissensbestände rekurrieren und diese somit rekonstruieren, zentral ist (vgl. Keller 2001; Keller et al. 2001). Eine diskursanalytische Herangehensweise an Jugendgewaltprävention beinhaltet die Analyse des Sprechens über Jugendgewaltprävention. Das Sprechen über Jugend bzw. Jugendliche gerät dabei insbesondere hinsichtlich seiner Adressierungsweisen in den Blick: Welche Positionen werden Jugendlichen im Sprechen über sie zugewiesen? Wie werden sie in diesem Sprechen über sie als soziale Gruppe bzw. Kategorie diskursiv hervorgebracht (vgl. Groenemeyer 2014: 55)? Das Sprechen über Jugendgewalt gerät hinsichtlich seiner Problematisierungsweisen (Devianzkonstruktionen) und das Sprechen über Jugendgewaltprävention hinsichtlich seiner Orientierungs- und Begründungsweisen (Legitimationsweisen) in den Blick, insofern als die Fachpersonen als Subjekte kriminalpolitischer Programmatik in ihrer Verantwortung angerufen werden, insbesondere in ihrer kollektiven Verantwortung. Wie bereits erwähnt, ist die Analyse durch Foucaults Vorlesungen zur Gouvernementalität angeregt und behandelt Konstruktions- und Legitimationsweisen von Wissen im Kontext von Jugendgewaltprävention. In »Sicherheit, Territorium, Bevölkerung« (2004a) beschreibt Foucault den historischen Wandel des Rechtssystems: Der Disziplinarmechanismus wird dabei sukzessive vom Sicherheitsdispositiv abgelöst. Diesen Wandel erklärt er am Beispiel des Strafsystems:

"Der Disziplinarmechanismus ist gekennzeichnet durch die Tatsache, daß im Inneren des binären Systems des Gesetzbuches eine dritte Person, der Schuldige, auftritt, und gleichzeitig erscheinen außerhalb, über den legislativen Akt, den das Gesetz aufstellt, und über den gerichtlichen Akt, der den Schuldigen bestraft, hinaus, eine ganze Reihe von angrenzenden polizeilichen, medizinischen, psychologischen usw. Techniken, die auf Überwachung, Diagnose, eventuelle Veränderung von Individuen usw. hinweisen." (Ebd.: 19) 
Die dem so beschriebenen Rechtssystem innewohnenden Technologien fokussieren die Tat des Schuldigen, indem sie über Maßnahmen der Bestrafung dessen Disziplinierung anpeilen. Schuldig ist der, der Verbotenes getan hat. Bestraft wird der, der schuldig ist. Schuld kann gesühnt werden. Verbote und Strafen sind im Gesetzbuch geregelt. Die Technologien des Sicherheitsdispositivs wenden sich hingegen von konkreter Schuld als Legitimation von Strafe ab. Auf das Schuldmotiv, das etwa Fjodor Michailowitsch Dostojewski in seinem Roman »Schuld und Sühne« zur Triebfeder der Handlung macht, ist kein Verlass mehr. Ins Zentrum der Strafe wird weder die Tat des Schuldigen noch die Schuld oder der Täter selbst gestellt, sondern die Wahrscheinlichkeit einer Tat, die primär über (Kriminalitäts-)Statistiken kontrolliert wird.

"Das Sicherheitsdispositiv also, welches, um es nun ganz pauschal zu sagen, das in Frage stehende Phänomen, nämlich den Diebstahl, ins Innere einer Reihe wahrscheinlicher Ereignisse eingliedert. Zweitens werden die Reaktionen der Macht im Hinblick auf dieses Phänomen in eine Kalkulation eingegliedert, in eine Kostenkalkulation. Und drittens wird, anstatt eine binäre Aufteilung zwischen dem Erlaubten und dem Verbotenen zu begründen, einerseits ein als optimal angesehener Mittelwert festgelegt, und andererseits werden Grenzen des Akzeptablen festgelegt, jenseits deren das nicht mehr passieren dürfte. Hier zeichnet sich also eine völlig andere Verteilung der Dinge und der Mechanismen ab." (Ebd.: 19f.)

Hieran werden drei Mechanismen deutlich, welche die Prävention als dem Sicherheitsdispositiv inhärente Logik beschreiben: Orientierung an Risiken, ökonomische Kalkulation und Normalisierung. Diese Mechanismen werden als zentrale Aspekte von Jugendgewaltprävention beschrieben (v.a. Kapitel 4.2). So geht mit der Fokussierung auf Risiken die Überlegung einher, dass Prävention dem Staat weniger kostet als Repression. Dabei wird die implizite Annahme gemacht, dass all diejenigen Personen, die straffällig werden würden, wenn nicht in Prävention investiert werde würde, durch die jahrelange Unterbringung und Therapierung sehr viel mehr kosten würden als die Investitionen in Prävention. Dies impliziert wiederum, dass davon ausgegangen wird, dass Prävention erfolgreich ist. Wie bereits erwähnt, beobachtet Foucault weiter den Wandel des Rechts- und des damit verbundenen Strafsystems sowie der diesen innewohnenden Technologien. Er begründet seine Beobachtungen über das Konzept der »Gouvernementalität«. Im 16. Jahrhundert, das gleichzeitig von einer Zersplitterung und Schwächung des Religiösen und der Entstehung europäischer Nationalstaaten geprägt ist, stellt sich die Frage des »Wie regieren?« neu. Besonders aufgrund der von Beginn an bestehenden Konkurrenz der europäischen Nationalstaaten verliert der »souveräne« Blick des Monarchen auf das Individuum zugunsten einer Kontrolle der Bevölkerung und Bevölkerungsgruppen an Bedeutung - ein Prozess, auf den Foucault insbeson- 
dere in »Die Geburt der Biopolitik« (2004b) eingeht. Neue Formen »pastoraler Macht« (Foucault 1994b) etablieren sich: Als zentrale Technik staatlicher Kontrolle entsteht die Statistik, da das Wissen um die Bevölkerung virulent wird. Es bildet sich eine Ökonomie der Bevölkerung, die nicht nur das »Leben«, sondern das »gute Leben« zum Ziel hat und in einem ganz spezifischen Verhältnis zum Liberalismus als politische Ökonomie steht (vgl. 2004b). Zentrales Element der Bevölkerungskontrolle wird die »moderne Familie «, in der Wissen, insbesondere medizinisches Wissen, direkt am menschlichen Leib praktiziert wird (vgl. Donzelot 1980). Diese Ausführungen verdeutlichen die Bedeutung der Triade Wissen, Macht und Subjekt aus einer diskurstheoretischen Perspektive im Anschluss an Foucault:

"Foucaults Studien kreisen immer um die Triade Wissen, Macht und Subjekt. Auch das Anliegen, aus dem heraus Foucault die Archäologie des Wissens verfasst, erschließt sich letztlich nur über die Schnittstelle dieser drei Elemente. Jede Arbeit, die sich an Foucault anlehnt, muss sich daher die Frage stellen, inwieweit eine Analyse der Aussagen und ihre Verknüpfung in Diskursformationen auch immer eine Analyse von Machtverhältnissen ist und inwiefern Wissen und Wahrheit auf Machtverhältnisse und Subjektivierungsweisen bezogen sind." (Schreiber 2011: 93)

Auch die hier vorliegende Arbeit stellt die Frage ins Zentrum, welche Subjektformen Jugendgewaltprävention hervorbringt. Sie nimmt an, dass sich Subjekte über den Gebrauch von Wissen konstituieren, das ihnen diesbezügliche Diskurse zur Verfügung stellen. Der Gebrauch dieses Wissens (re-)konstruiert grundlegende Machtverhältnisse, die das Handeln von Fachpersonen und Jugendlichen prägen. Oben beschriebene Ausführungen werden in Forschungsarbeiten über Prävention aufgegriffen. Eine der grundlegenden Arbeiten, die sich mit dem Verhältnis politisch-verwalterischer Logik von Prävention und sozialer Praxis präventiven Handelns beschäftigt, ist Robert Castels Aufsatz »Von der Gefährlichkeit zum Risiko« in Manfred Max Wambachs Sammelband zur »Logik von Prävention und Früherkennung « (Castel 1983). Castel beobachtet, dass Präventionsstrategien sich historisch dahingehend änderten, als sie weniger konkrete Gefahren zum Gegenstand ihrer Sorge machten, sondern vielmehr abstrakte Risiken. Vor diesem Hintergrund geht er der Fragestellung nach, inwiefern die veränderten, an Risiken ansetzenden Präventionsstrategien, zu einem Verlust der Unmittelbarkeit der Beziehung zwischen Sozialarbeiterin bzw. Sozialarbeiter und Adressatin bzw. Adressat führen, woran die Forschungsfrage der hier vorliegenden Arbeit anschließt. Die neuen, Risiken fokussierenden Präventionsstrategien beziehen sich weniger auf konkrete Individuen, die als gefährlich etikettiert sind, sondern vielmehr auf Risikomerkmale, die bestimmte Personen oder Personengruppen, wie etwa Kinder oder Jugendliche, allgemein als gefährliche oder gefährdete oder gleichzeitig als ge- 
fährliche und gefährdete soziale Gruppe zuschreibbar machen. Diese Personen oder Personengruppen erkennen sich in diesem Moment des »Zuschreibbar-gemacht-Seins « selbst nicht als gefährlich an. Dieser Status entspricht, um es mit Erving Goffman auszudrücken, dem »Diskreditierbar-Sein« und nicht dem »Diskreditiert-Sein«:

"The term stigma and its synonyms conceal a double perspective: does the stigmatized individual assume his differentness is known about already or is evident on the spot, or does he assume it is neither known about by those present nor immediately perceivable by them? In the first case one deals with the plight of the discredited, in the second with that of the discreditable." (Goffman 1963: 4)

Allerdings ist, wie Goffman beschreibt, der Zustand des »DiskreditierbarSeins « als eine Form der Stigmatisierung und damit als fortwährende Bedrohung der eigenen Identität. Auf politisch-verwalterischer Ebene bedeutet dies, dass >repressive Strategien< (wie z.B. das Wegsperren oder Wegweisen konkreter als gefährlich etikettierter Personen) von präventiven Strategien verdrängt würden. Diese bestünden darin, Wissen über Wahrscheinlichkeiten des Auftretens bestimmter unerwünschter Handlungsweisen zu generieren, was Castel als eine »neue Art und Weise der Überwachung: die systematische Feststellung und Erfassung « (Castel 1983: 60) bezeichnet. »Die präventiven Politiken befassen sich nicht mehr in erster Linie mit Individuen, sondern mit Faktoren, mit statistischen Korrelationen heterogener Elemente.« (Ebd.: 61) Im Anschluss daran stellt sich die Frage, wie sich Fachpersonen im Kontext von Jugendgewaltprävention, insbesondere der Sozialen Arbeit, die einen implizit oder explizit vermittelten Präventionsauftrag zu erfüllen haben, zu »präventiven Politiken « positionieren. Nach Castel ist davon auszugehen, dass die historisch etablierte Kluft zwischen politisch-verwalterischer und sozialpädagogischer Praxis reproduziert wird und sich vergrößert. Im Anschluss an Foucault und Castel ist Prävention in modernen Staaten als zentrale Machttechnologie $\mathrm{zu}$ verstehen, die - in Risiko- und Sicherheitsdiskurse eingebettet - nicht »mehr das Individuum selbst zum Gegenstand der Kontrolle« hat, sondern »die Regulierung von Bevölkerungen, Populationen oder Kollektiven, in denen sich Handlungsformen statistisch verteilen « (Groenemeyer 2015: 21), zum Ziel hat. Diese Beobachtung wird von verschiedenen Autorinnen und Autoren aufgegriffen, die eine Ausweitung präventiver Politik zulasten sozialstaatlicher und rechtsstaatlicher Politik konstatieren. Daran problematisieren sie, dass Strategien der Integration, Rehabilitation und Resozialisierung Strategien der Kontrolle weichen, die an das Sicherheitsbedürfnis der Bevölkerung appellieren (vgl. Denninger 1988: 10; Garland 2008: 36; Haffke 2005: 17ff.; Krasmann 2011: 54; Lindenau 2012: 325; Lindenau/Münkler 2012: 64). Mathias Lindenau spricht hierbei von einer »präventiven Zäsur«, insofern als die »Omnipräsenz 
[von Prävention] als Indiz dafür gewertet werden kann, dass die Prävention zur neuen Patentlösung einer verunsicherten Gesellschaft avanciert ist« (Lindenau 2012: 325). Damit geht die These einher, dass Politik und Verwaltung weniger konkrete als gefährlich etikettierte Personen adressieren und über die Konstruktion »omnipräsenter « Risiken (über Wissensproduktion) bestimmte Bevölkerungsgruppen, wie z.B. »die Jugend«, unter Generalverdacht stellen. Auch der Begriff der »Präventionslandschaft« verweist auf eine mit Prävention einhergehende Institutionalisierung von Macht und Wissen: »PräventionsProgramme und -Maßnahmen werden zu einem weiten Feld von organisierter Wissensproduktion und -verwertung. Präventionsketten, Präventionstage, Präventionsnetzwerke und Präventionsforen wurden organisiert und verwaltet.« (Widersprüche 2016: 3; vgl. auch Cremer-Schäfer 2016: 19) Die zentrale Forschungsfrage dieser Arbeit zielt nun darauf ab, inwieweit sich Fachpersonen im Kontext von Jugendgewaltprävention, insbesondere Sozialer Arbeit, an Risikodiskursen orientieren.

"Diese neuen Strategien sind mit der Auflösung des Begriffs des Subjekts oder des konkreten Individuums verbunden, der durch einen Komplex von Faktoren, die Risikofaktoren, ersetzt wird. Wenn das zutrifft, so bringt eine derartige Transformation bedeutsame Implikationen für die Praxis mit sich. Das Wesentliche bei der Intervention ist nicht länger die unmittelbare Beziehung, die Konfrontation zwischen beispielsweise einem Betreuer und einem Betreuten, einem Helfer und einem Hilfeempfänger, einem Fachmann und seinem Klienten. Entscheidend ist vielmehr die Konstruktion von Populationen, die auf einer Kombination abstrakter, generell risikoträchtiger Faktoren beruht. Durch diese Verschiebung entsteht ein krasses Mißverhältnis zwischen dem Standpunkt des Fachpersonals auf der einen Seite und dem der Verwaltungskräfte, die diese Gesundheitspolitiken definieren und verwirklichen, auf der anderen." (Castel 1983: 51)

Es werden nicht nur diesbezügliche Konsequenzen für die professionelle Praxis, sondern auch für die Jugendlichen in den Blick genommen. Auch der Diskurs über Jugendgewaltprävention ist als kriminologisch geprägter Risikodiskurs anzusehen, der sich stark über Ökonomie und Wissenschaft, insbesondere der Kriminologie, zu legitimieren sucht sowie über utopische und dystopische Metaphern, die Szenarien omnipräsenter Bedrohung durch Gewalt in der Öffentlichkeit schaffen. Es wird der Frage nachgegangen, wie sich soziale Praxis, insbesondere im Kontext Sozialer Arbeit, zu einem spezifischen kriminalpolitischen Programm im Zusammenhang von Jugendgewaltprävention in der Schweiz, das in Beziehung zu Risiko- und Sicherheitsdiskursen steht, verhält. Fokussiert werden dabei, wie bereits erwähnt, »diskursive Praktiken« im Kontext von Jugendgewaltprävention und demnach das Sprechen über Jugendgewaltprävention sowohl im Rahmen kriminalpolitischer Programmatik als auch von Interviews mit Adressatinnen und Adressaten eben dieses krimi- 
nalpolitischen Programms, die als über Diskurse bzw. diskursive Formationen erzeugte »Subjekte« in den Blick geraten. Diese Verknüpfung bringt methodologische und methodische Herausforderungen bezüglich der Integration interpretativer Methoden in diskursanalytische Vorgehensweisen mit sich (vgl. Angermüller 2014; Bender/Eck 2014; Keller 2004; König 2008), die in den Kapiteln 2.4 und 3 ausgeführt werden. Damit bildet das Interesse an Legitimations- und Wirkungsweisen eines in der Schweiz angesiedelten kriminalpolitischen Programms bezüglich Jugendgewaltprävention den Ausgangspunkt der hier vorliegenden Arbeit. Die Analyse geht jedoch über die programmatische Ebene hinaus und beleuchtet handlungsrelevante Anschlussmöglichkeiten aus der Perspektive von Fachpersonen im Kontext von Jugendgewaltprävention, die in den programmatischen Aussagen in ihrer Verantwortung angerufen werden. Eine Frage der Analyse betrifft im Gegensatz zu »klassischen« Programmanalysen - als besonders relevant ist hier eine Studie von Verena Schreiber zu kommunaler Kriminalprävention herauszustellen (vgl. Schreiber 2011) - nicht nur, welches »Verhalten den Menschen zugetraut wird; wie sie sich verändern sollen, welche Zumutungen das präventive Programm von ihnen abverlangt [...]. Wie werden die Menschen von der kommunalen Kriminalprävention angerufen, sich als verantwortliche Bürger für eine sichere Gesellschaft zu begreifen?« (ebd.: 107), sondern auch, und vielmehr, wie sich Fachpersonen, insbesondere aus der Sozialen Arbeit, zu derlei Anrufungen positionieren. Die Analyse kriminalpolitischer Programme ist soziologisch insofern relevant, als diese soziale Ordnung ermöglichen, indem sie Relevanzen festlegen, Problembereiche definieren, Risikogruppen konstruieren, bestimmte Personen oder Personengruppen als Adressatinnen und Adressaten in ihrer Verantwortung anrufen und somit letztlich Wissen konstruieren, das in soziale Praktiken Eingang findet. Dabei reduzieren die damit einhergehenden Institutionalisierungsprozesse Kontingenz, d.h., gewisse Aspekte des Wissens bleiben unbelichtet - eine Intransparenz, die gewissermaßen als funktional erachtet werden kann, insofern sie bestehende Machtverhältnisse reproduziert (vgl. Popitz 2003). Es lässt sich also behaupten, dass kriminalpolitische Programme als Institutionalisierungsprozesse angesehen werden können, die (Un-)Sichtbarkeiten erzeugen und soziale Praxis strukturieren. Leon Hempel, Susanne Krasmann und Ulrich Bröckling sprechen hierbei von »Sichtbarkeitsregimen« als

"technische und soziale Arrangements, die Ordnung stiften und stabilisieren, Gefährdungen abwehren und Abweichungen korrigieren sollen und selbst eine Ordnung des Beobachtens und beobachtet Werdens, des Zeigens und Verbergens etablieren. Sie wirken gleichermaßen auf das Handeln von Beobachtern wie Beobachteten ein, lenken Blicke und dirigieren Aufmerksamkeiten; sie holen Verborgenes ans Licht und sorgen dafür, dass es den Blicken entzogen bleibt; sie vergrößern Winzigkeiten oder zoomen 
Weitentferntes heran und machen es so für regulierende Zugriffe erreichbar; sie aggregieren Einzelbeobachtungen und schaffen dadurch erst bestimmte Objekte, z.B. statistische Normalverteilungen oder Risikogruppen. Sie definieren so die Probleme, zu deren Lösung sie installiert werden." (Hempel/Kasmann/Bröckling 2011: 8f.)

Im Anschluss daran fragt die hier vorliegende Arbeit, inwiefern die Fachpersonen im Kontext von Jugendgewaltprävention in ihren je spezifischen institutionellen und organisatorischen Settings an sozialprogrammatisch erzeugte (Un-)Sichtbarkeiten und (Un-)Sagbarkeiten anschließen. Was bedeutet dies für ihre subjektiven Präventionsverständnisse, die diesen zugrunde liegenden Problematisierungsweisen und die Jugendlichen, die sie wiederum adressieren? In den weiteren Analysefokus geraten dabei nicht »Praktiken im Modus ihres Vollzugs « (Ott/Wrana 2010: 158), sondern Praktiken im Sinne von »Sprechen über« in einem kriminalpolitischen Bezugsrahmen sowie in Interviews, die als Diskurs(-re-)produktion (vgl. Bublitz et al. 1999: 14) angesehen werden können: Die Fachpersonen im Kontext von Jugendgewaltprävention werden als solche durch die Forscherin adressiert, die sich selbst in kriminalpolitischen Kontexten bewegt, also auf programmatische Inhalte zurückgreift, um Interviews zu einem spezifischen Thema, hier das der Jugendgewaltprävention, zu realisieren. Diese Problematisierung greifen die Kapitel 3.1 und 4.1 auf. Das Besondere am »Nationalen Präventionsprogramm Jugend und Gewalt« ist, dass es sich nicht an die breite Öffentlichkeit richtet, sondern an ein Fachpublikum, insbesondere aus der Sozialen Arbeit, das jedoch sehr breit gefächert ist. Deshalb und aufgrund seiner Ziele ist es mit der in Deutschland als kommunale Prävention bezeichneten Prävention, für die Analysen vorliegen (vgl. Schreiber 2011; Berner/Groenemeyer 2003), nur bedingt vergleichbar.

"Die Initiativen für ressortübergreifende Kriminalprävention kommen in vielen Fällen 'von unten`, ihre Durchführung hing und hängt an einzelnen Einrichtungen oder am Engagement einzelner Personen. Deshalb unterscheiden sich die Modelle und die Entstehungsprozesse je nach den lokalen Traditionen, Strategien und Gegebenheiten von Kommune zu Kommune. Die Heterogenität der verschiedenen kriminalpräventiven Projekte spiegelt sich in den vielfältigen Bezeichnungen für die Präventionsmaßnahmen auf Länder- oder auf kommunaler Ebene. Über die verschiedenen Bezeichnungen hinweg haben diese Einrichtungen eine Gemeinsamkeit: Unter Berufung auf Slogans wie ,Kriminalprävention ist eine gesamtgesellschaftliche Aufgabe ‘ propagieren sie die Vernetzung von Akteuren zur Bündelung der verschiedenen Kapazitäten und Kompetenzen. Dabei wird in einem kommunitären Gemeinschaftsdiskurs an die gesellschaftliche Selbstorganisation und an die Beteiligungsbereitschaft der Bürger und Bürgerinnen appelliert. Die Aktivitäten, die typischerweise im Rahmen der kommunalen Kriminalprävention entfaltet werden, sollen der Begründungsrhetorik nach Sicherheit und Lebens- 
qualität im öffentlichen städtischen Raum herstellen oder zumindest sichern." (Berner/ Groenemeyer 2003: 85)

Das »Nationale Präventionsprogramm Jugend und Gewalt« zielt zwar auch auf Vernetzung ab, strebt dabei jedoch weniger eine (verstärkte) Föderalisierung von Maßnahmen an, sondern vielmehr eine schweizweite Standardisierung dieser - Macht, Wissen, Expertise und Verantwortung sollen also zentralisiert und institutionalisiert werden. Jugendgewaltprävention wird nicht als gesamtgesellschaftliche Aufgabe, sondern als hochprofessionelle Aufgabe kommuniziert, die ganz spezifischen Regeln folgen soll. Prävention lässt sich jedoch in beiden Kontexten als politische Programmatik verstehen (vgl. Lindenau/ Münkler 2012: 64), die bestimmte Phänomene problematisiert, damit als bearbeitbar konstruiert und die es in ihren Wirkungsweisen aus programmanalytischer Perspektive zu untersuchen gilt. Programme

"stellen bestimmte Formen gesellschaftlicher Problematisierung dar und schon auf diese Weise formen sie die Realität, die sie in ihrer Sprache und Denkweise zuschneiden. Indem sie Probleme definieren und Ziele formulieren [...] legen Programme Gegenstandsbereiche und die Relevanz von Gegenständen fest, und sie zeigen die Richtung an, auf die hin die Realität zu verändern ist [...]. Nicht ob und wie Programme richtig umgesetzt werden, gilt es daher zu eruieren, auch nicht, ob die Ziele, die sie formulieren, prinzipiell wünschenswert oder ihre Lösungsvorschläge geeignet sind. In diesem Sinne ist die Fragestellung nicht normativ, wohl aber darin, dass sie die Bedingungen auszuloten sucht, unter denen bestimmte Normen wirksam werden, und dass sie untersucht, mit was für einer Gesellschaft wir es zu tun haben, die bestimmte Probleme als die ihren akzeptiert, in bestimmter Weise diskutiert und traktiert. Gegenstand der Analyse sind die Denk- und Sichtweisen, die Programme vermitteln, und die Frage ist, wie diese Sichtweisen sich in Technologien des Regierens, also in systematischen Praktiken der Menschenführung realisieren." (Kessl/Krasmann 2005: 231f.)

Gegenstand der hier vorliegenden Arbeit ist somit das Sprechen über Jugendgewalt im Kontext von Jugendgewaltprävention im Rahmen einer kriminalpolitischen Programmatik und Interviewpraxis. Jugend und Gewalt, so die Annahme, können aufgrund ihrer sozialen (und historischen) Konstruiertheit nicht als analytische Instrumente in der hier vollzogenen Analyse dienen (vgl. Stehr 2009: 107), sondern geraten im »Sprechen über« als Gegenstände der Untersuchung in den Blick. Als analytisches Gerüst dient vielmehr die Triade Subjekt, Macht und Wissen im Anschluss an Foucault, die das Kapitel 2.3 näher beleuchtet. Jugendgewalt, so die These, wird im Sprechen über Jugendgewaltprävention als Deutungsmuster diskursiv rekonstruiert und ist dabei als diskursives Moment zentral für gesellschaftliche Ordnungsbildung. Für die Analyse der Orientierungsweisen verschiedener Fachpersonen im Kontext von 
Jugendgewaltprävention an kriminalpolitischer Programmatik ist, wie bereits erwähnt, die Beobachtung historischer Veränderungen von Präventionsstrategien relevant, mit der eine Kluft zwischen politischer Verwaltung und sozialer Praxis einhergeht. Vor diesem Hintergrund stellt sich die Frage des Verlustes einer Unmittelbarkeit der Beziehung zwischen Fachpersonal und Klientel (vgl. Castel 1983: 51; Schreiber 2011: 58f.). Prävention kann dabei als zentrale Technik verstanden werden, die über Risiko- und Sicherheitsdiskurse vermittelt wird, wobei konstatiert wird, dass Bedrohungen zunehmend über antizipierte Gefahren und immer weniger über gefährliche Individuen verhandelt werden (vgl. Groenemeyer 2015: 21) und in der Konsequenz die »Bearbeitung abweichenden Verhaltens und sozialer Probleme zu einem Risikomanagement wird, das sich weniger auf die Bearbeitung der Probleme von Betroffenen bezieht« (ebd.: 30) und mehr auf die Bearbeitung abstrakter Risiken. Die beobachtete »Kluft« zwischen Praktiken im Kontext Sozialer Arbeit und Politik und die damit einhergehende Distanzierung zwischen Fachpersonal und Adressatinnen und Adressaten wird also auf die Fokussierung der Bedeutung von Risiken zurückgeführt. Prävention ist in diesem so beschriebenen Szenario (Sicherheitsdispositiv) als eine zentrale Technologie zu verstehen, insofern sie das Wahrscheinliche zum Gegenstand hat, das sie eigentlich vermeiden will. So gesehen ist das, was der Sicherheitsstaat vermeiden will, eben genau das, was er selbst hervorbringt (vgl. Brüchert 2010: 273; Schreiber 2011: 149f.). Durch diese Logik der Selbstreferentialität reproduziert sich dieses System letztlich selbst. Empirische und an der Schweiz orientierte Forschungsarbeiten hinken diesen Beobachtungen allerdings hinterher, insbesondere auch bezüglich des spezifischen Kontexts von Jugendgewaltprävention. Aus dieser Perspektive, die historische Prozesse sowie Prozesse, die politische, verwalterische und sozialpädagogische Praxis betreffen, in den Blick nimmt, lassen sich kriminalpolitische Fokussierungen auf sog. evidenzbasierte Maßnahmen bezüglich Prävention als Praktiken der Kontrolle beschreiben. Die hier vorliegende Arbeit verknüpft diese Perspektive auf Prävention mit aktuellen kindheits- und jugendsoziologischen Fragestellungen, die insofern an Castels Beobachtungen anschließen, als sie das Sprechen über Kindheit und Jugend als soziale Gruppe in politischen wie wissenschaftlichen Diskursen kritisieren. Gegenstand der Analyse ist hierbei das Sprechen über »Jugendgewaltprävention « im Rahmen von Interviews mit Fachpersonen, die im Bereich von Jugendgewaltprävention tätig sind. Die Interpretationen der Interviews werden in Bezug gesetzt zu Interpretationen von Dokumenten - ein Transkript und zwei Protokolle -, welche die Forscherin im Rahmen von Konferenzen, veranstaltet vom »Nationalen Präventionsprogramm Jugend und Gewalt«, angefertigt hat. Dieses Programm hat primär zum Ziel, Jugendgewaltprävention u.a. durch flächendeckende Implementierung sog. »evidenzbasierter Projekte« schweizweit zu standardisieren und ruft dabei unterschiedlichste Fachbereiche in ihrer Ver- 
antwortung an. Vernetzung, Austausch und die Schaffung einer gemeinsamen Wissensbasis sollen zu einer »verbesserten Prävention« beitragen. Die Metapher, dass alle im selben Boot sitzen, ist zentral und verweist auf die Bedeutung utopischer und dystopischer Szenarien für die Legitimation kriminalpräventiver Strategien (vgl. Liell 2002: 6; Schreiber 2011: 145f.). Das Programm appelliert an Fachpersonen verschiedenster Tätigkeitsbereiche Sozialer Arbeit, aber auch an Polizei, Justiz und Medizin, gemeinsam zusammenzuarbeiten, sich zu vernetzen und Wissen auszutauschen. Jugendgewalt tritt in der vorliegenden Arbeit insofern als zentraler Gegenstand diskursiver Praktiken im Kontext von Jugendgewaltprävention in den Vordergrund, als dessen Problematisierung als Legitimation politischer und sozialpädagogischer Praxis angesehen werden kann. Angeschlossen wird hierbei auch an aktuelle theoretische Auseinandersetzungen mit dem Gewaltbegriff, insbesondere in der Soziologie. Diese beklagt einen Mangel an theoretischer Reflexion des Gewaltbegriffs, insoweit als Sozialtheorien Gewalt primär als einen zu problematisierenden Gegenstand am Rande der Sozialität verorten. Die Identifikation mit einer »gewaltfreien Moderne« geht mit Sensibilisierungs- und Skandalisierungsprozessen einher, die auch eine Kritik der Gewalt begünstigen (vgl. Liell 2002: 8f.). Sich kritisch positionierende Ansätze begreifen Gewalt hingegen als zentrales Charakteristikum gesellschaftlicher Ordnungsbildung, insbesondere moderner Gesellschaften (vgl. Baumann 1992; Garland 2008; Han 2011; Hugger 1995; Koloma Beck/Schlichte 2014; Krasmann 1997; Liell 2002). Die hier vorliegende Arbeit schließt insoweit an diese Kritik an, als sie davon ausgeht, dass Gewalt als diskursiv erzeugter Gegenstand zentral für die Stabilität gesellschaftlicher Machtverhältnisse ist, indem er in Handlungspraktiken reproduziert wird und sich auf vulnerable Bevölkerungsgruppen bezieht. Das im Kontext der hier vorliegenden Arbeit primär interessierende Wissen ist das Wissen über Jugendgewalt und daran anschließende Durchführungsweisen und Ziele von Prävention. Es wird davon ausgegangen, dass das Wissen über Jugendgewalt die in bestimmten Kontexten relevanten Probleme mitkonstituiert, woran Präventionskonzepte anschließen. Gegenstand der Analyse ist also die »pädagogische Blickpraxis« (Schmidt 2015: 107) und auf welche Weise sie Devianz hervorbringt. Jugendgewalt, so die These, wird als Deutungsmuster diskursiv reproduziert und somit als diskursives Moment zentral für gesellschaftliche Ordnungsbildung. Somit ist Diskurstheorie im Anschluss an Foucault als sozialkonstruktivistische Theorie zu begreifen, derzufolge soziale Phänomene sozialen Herstellungsprozessen unterliegen, was auch von der kritischen Kriminologie aufgegriffen wird:

"Eine Grundannahme kritischer Kriminologie ist, das Kriminalität kein beobachtbares Verhalten einzelner Täter darstellt, deren Ursachen erforscht werden können und keine Eigenschaft bildet, die einem individuellen Verhalten inhärent ist. Kriminalität gilt 
als Produkt vielfältiger Zuschreibungsprozesse und ist damit eine gesellschaftliche Erscheinung, die nur als gesellschaftlicher Konstitutionsprozess rekonstruiert werden kann. Zentral für die kritische Kriminologie ist demnach der Prozess der Herstellung von Kriminalität." (Althoff 2002a: 47)

Die hier vorliegende Arbeit rekonstruiert nun Jugendgewalt als Produkt gesellschaftlicher Konstitutionsprozesse. Mittels offener leitfadengestützter Interviews mit Fachpersonen aus unterschiedlichen Bereichen der Jugendgewaltprävention wird der Frage nachgegangen, inwiefern sie sich in ihren Problematisierungsweisen und Präventionskonzepten an kriminalpolitischer Programmatik orientieren. Das Interesse am Verhältnis von sozialer Praxis und kriminalpolitischer Programmatik ist hochaktuell, bringt allerdings methodologische wie methodische Herausforderungen mit sich, wie beispielsweise die Integration interpretativer Verfahren in diskursanalytische Herangehensweisen. Empirisch geraten bei der Analyse diskursiver Praktiken auf der Ebene des »Nationalen Präventionsprogramms Jugend und Gewalt« und auf der Ebene der Interviews jedoch unterschiedliche Adressierungsweisen und damit Prozesse der Subjektivierung und Objektivierung in den Blick. Werden auf der Ebene des Programms die Fachpersonen als verantwortliche Subjekte angerufen und »die Jugendlichen« bzw. »die Jugend« als zuschreibungsfähige soziale Gruppe adressiert, kommt es auf der Ebene der Interviews durchaus zu Aussagen über Positionierungen Jugendlicher als verantwortliche Subjekte.

\section{2 ÜBER DAS VERHÄLTNIS VON DISKURS UNd diskURsiven Praktiken}

Im Folgenden werden grundlegende diskurstheoretische Begriffe expliziert, die für die Analyse der hier vorliegenden Arbeit zentral sind. Die Unterscheidung zwischen »Diskurs « und »diskursiven Praktiken« ist insofern relevant, als es nicht »den einen« Diskurs um Jugendgewaltprävention gibt, sondern es sich um ein Feld handelt, in dem verschiedene Fragmente und Praktiken in einem Zusammenhang stehen. Dieser muss analytisch getrennt werden, was methodologische und methodische Implikationen zur Folge hat. Diskursanalytische Ansätze beziehen sich in der Regel auf Foucaults Diskursbegriff, wobei er keine exakte Definition dessen anbietet. Allgemein gesprochen schließen Diskurse nach Foucault jegliches »Gesagte « ein. Dabei werden Individuen dazu bemächtigt, selbst etwas zu »sagen« bzw. über etwas zu sprechen, indem ihnen Wissen zur Verfügung steht, auf das sie sich in ihren Äußerungen beziehen können. Diskurse lassen sich folglich als Praktiken des »Sprechens über« beschreiben. Eine »Diskursivierung des Sexes« (Foucault 1979: 21) im Sinne einer sprachlichen (diskursiven) »Vermehrung« oder auch »Verdich- 
tung« des Sexes, so zeigt Foucault, befähigt Individuen dazu, sich selbst als sexualisiert zu erfahren und gesellschaftlich anerkennbar zu machen. Der Gebrauch des Wissens über einen Gegenstand, dort der Sexualität, bemächtigt sie dazu, über Sexualität zu sprechen und Sexualität als solche zu praktizieren. Nach Foucault bedingt die Fähigkeit, über etwas zu sprechen, verschiedene »Aussagen« aufeinander zu beziehen:

"Die in ihrer Form verschiedenen, in der Zeit verstreuten Aussagen bilden eine Gesamtheit, wenn sie sich auf das ein und dasselbe Objekt beziehen. [...] Die Geisteskrankheit ist durch die Gesamtheit dessen konstituiert worden, was in der Gruppe all der Aussagen gesagt worden ist, die sie benannten, sie zerlegten, sie beschrieben, sie explizierten, ihre Entwicklungen erzählten, ihre verschiedensten Korrelationen anzeigten, sie beurteilten und ihr eventuell die Sprache verliehen, indem sie in ihrem Namen Diskurse artikulierten, die als die inren gelten sollten. Aber mehr noch: Diese Gesamtheit von Aussagen ist weit davon entfernt, sich auf ein einziges Objekt zu beziehen, das ein für allemal gebildet ist, und es unbeschränkt als ihren Horizont unerschöpflicher Idealität zu bewahren; das Objekt, das von den medizinischen Aussagen des 17. oder 18. Jahrhunderts als ihr Korrelat gesetzt worden ist, ist nicht identisch mit dem Objekt, das sich durch die juristischen Urteilssprüche und die polizeilichen Maßnahmen hindurch abzeichnet." (Foucault 1994a: 49f.)

Die Überlegung, Diskurse ließen sich über Aussagen beschreiben, die sich jedoch nicht auf ein und dasselbe Objekt beziehen, führt zu der Frage, wie sich Diskurse und ihre Wirkungsfelder als Untersuchungsgegenstand eingrenzen lassen, insbesondere empirisch:

"Ebenso sind alle Gegenstände des psychopathologischen Diskurses seit Pinel oder Esquirol bis zu Bleuler hin verändert worden: es sind nicht dieselben Krankheiten, um die es sich dort oder hier handelt; es sind nicht dieselben Irren, um die es geht. Aus dieser Vielfalt der Objekte könnte man, müßte man vielleicht schließen, daß es nicht möglich ist, den 'den Wahnsinn betreffenden Diskurs als eine gültige Einheit für die Konstituierung einer Gesamtheit von Aussagen zuzulassen. [...] Die Einheit der Diskurse über den Wahnsinn wäre nicht auf die Existenz des Gegenstands ,Wahnsinn ‘ oder die Konstitution eines einzigen Horizontes von Objektivität gegründet; es wäre das Spiel der Regeln, die während einer gegebenen Periode das Erscheinen von Objekten möglich machen: [...] Wenn es eine Einheit [des Diskurses] gibt, so ist deren Prinzip keine determinierende Form von Aussagen; wäre es nicht eher die Gesamtheit der Regeln, die gleichzeitig oder nacheinander reine perzeptive Beschreibungen, aber auch durch Instrumente vermittelte Beobachtungen, Erfahrungsprotokolle aus Laboratorien, statistische Berechnungen, epidemiologische oder demographische Feststellungen, institutionalisierte Regelungen, therapeutische Vorschriften möglich gemacht haben?" (Ebd.: 50ff.) 
Diskurse sind im Anschluss an Foucault folglich weniger als klar strukturierte Aussagen zu verstehen, die zielgerichtet wirken, sondern eher als Regelwerke, die bestimmte Aussagen ermöglichen bzw. dazu befähigen - Aussagen, die anschlussfähig sind und aufgegriffen werden können. Bezüglich des Ziels, Jugendgewalt im Kontext von Jugendgewaltprävention zu rekonstruieren, werden im Anschluss an Foucault folgende Schlüsse zur Eingrenzung des Forschungsgegenstands gezogen: Unter Diskursen werden in einem Raum kontinuierlich ermöglichte (und damit gleichzeitig verunmöglichte) Aussagen bezüglich eines Gegenstands verstanden. Diskurse schließen also sowohl Sagbares als auch Unsagbares ein. Diskurse über Jugendgewaltprävention ermöglichen (und verunmöglichen) damit Aussagen bezüglich Jugendgewaltprävention (Jugend, Gewalt, Prävention, Jugendgewalt, Gewaltprävention). Insofern sind die relevanten Aussagen nicht nur auf der Ebene des »Nationalen Präventionsprogramms Jugend und Gewalt«, sondern auch auf der Ebene der Interviews diskursiv ermöglicht (und verunmöglicht) und als solche anschlussfähig (oder eben nicht). Indem die Interviews mit Fachpersonen im Kontext von Jugendgewaltprävention eben diesen (Un-)Möglichkeitsraum von Aussagen eröffnen, bedienen sich die Teilnehmenden bestehender Diskurse. Damit kann diese Erhebungsmethode als eine Technik angesehen werden, die Diskurse (re-)produziert. Diese Erkenntnis muss in die Interpretation der Interviews miteinfließen. Analysegegenstand der hier vorliegenden Arbeit sind also im diskursiven Kontext von Jugendgewaltprävention ermöglichte und verunmöglichte Aussagen bezüglich Jugendgewaltprävention, die auf der Ebene des Programms und der Ebene der Interviewpraxis in Bezug auf Aspekte von Macht, Wissen und Subjekt untersucht werden. Folglich prägt zunächst eine methodische Reflexion dieses Forschungsgegenstands im Sinne einer Reflexion der Forschungspraxis, die sich einer Kombination aus diskursanalytischer Methodologie und interpretativer Sozialforschung auf Basis von Interviews bedient, den Beginn der Analyse. Was machen Forschende, wenn sie aus diskursanalytischer Perspektive Interviews erzeugen? Wie ist es den Teilnehmenden möglich bzw. wie wird es ihnen ermöglicht, über Jugendgewaltprävention zu sprechen? Als Analyseperspektive wird dabei auf den Begriff der Subjektivierung rekurriert, der in den Blick nimmt, wie es möglich ist bzw. ermöglicht wird, als jemand über einen Gegenstand zu sprechen. Dabei wird auch reflektiert, in welcher Beziehung die Praktiken des Sprechens in den Interviews mit den Praktiken des Sprechens auf kriminalpolitischer Ebene stehen. Subjekt-Sein wird durch Anrufungsprozesse ermöglicht, die jedoch nicht mit Adressierungsprozessen gleichzusetzen sind (vgl. Reh/Ricken 2012: 42). Allerdings ist davon auszugehen, dass die Interviewerin durch Adressierungsweisen Anrufungen transportiert und damit Anschlussmöglichkeiten erzeugt (vgl. Bröckling 2013: 55). Beispielsweise ermöglicht die Anrufung »Weil Sie Expertin sind, wissen Sie, wie man gute Präventionsarbeit leistet!« im Kontext von Jugendgewaltpräven- 
tion verschiedenste Positionierungen (vgl. König 2008: 4790). Im Anschluss an diesen ersten Analyseschritt, der eine Reflexion von Adressierungsweisen einschließt und damit ein erstes Verständnis von Subjektpositionen zulässt, wird der Analysefokus auf den Gegenstand des Sprechens - Jugendgewaltprävention - gelenkt. Vor dem Hintergrund einer ersten Untersuchung bezüglich der Subjektpositionierungen geben weitergehende, primär fallspezifische Interpretationen Aufschluss über Problematisierungsweisen von Jugendgewalt und die daran anschließenden Präventionsverständnisse sowie Subjektverständnisse bezüglich Jugendlicher, die schließlich in themenspezifischen Interpretationen fokussiert werden. Fall- und themenspezifische Interpretationen greifen damit ineinander, insofern als fallvergleichende Interpretationen Aufschluss über die relevanten Themen und Begründungsweisen im Sinne kollektiv geteilter Orientierungsmuster liefern. Die dokumentarische Methode wird dabei als geeignetes Instrument angesehen, weil sie in der analytischen Unterscheidung eines »kommunikativen Wissens« und eines »konjunktiven Wissens« die semantische Ebene eines Gesprächs mitreflektiert (vgl. Bohnsack/Nentwig-Gesemann/Nohl 2001). Angeregt durch Harold Beckers Etikettierungsansatz (1963), der durch Malcom Spector und John Kitsuse (1977) weiterentwickelt wurde, werden die Interviews dahingehend untersucht, welche Handlungsformen auf welche Weise, d.h. v.a. vor dem Hintergrund welcher normativen Erwartungen, als problematisch etikettiert werden (vgl. Schmidt 2015). Schließlich wird untersucht, inwieweit diese Problemzuschreibungen und damit verbundenen Normalitätsvorstellungen diskursiv vorstrukturiert sind. Denn auch wenn der Etikettierungsansatz und dessen v.a. empirische Rezeptionen interaktionistisch geprägt sind, lässt sich, dieser Tradition folgend, davon ausgehen, dass »strukturelle Bedingungen«, gemeinsam ausgehandelte Regeln und »kulturell etablierte« Normen die Wahrnehmung von Kriminalität formen (vgl. Baratta 1986; Cremer-Schäfer 1998; Smaus 1986). Laut Wolfgang Stangl ist die »zentrale Aussage dieser Konzepte, daß die Reaktion der Kontrollinstanzen auf abweichendes Verhalten deviante Identitäten entweder verfestigt oder überhaupt entstehen lässt« (Stangl 1986: 122). Der Fokus ist hier jedoch primär diskursanalytisch, da die Verwobenheit professioneller Praxis und politischer Diskurse erforscht werden soll und dabei über die zentrale Kategorie des Subjekts Aspekte von Macht und sozialer Ungleichheit fokussiert werden sollen. Die Ermöglichung des Sprechens über Jugendgewaltprävention kann im Anschluss an Foucault, wie bereits erwähnt, als Bemächtigung, über etwas sprechen zu können, angesehen werden. Diskurse, verstanden als Aussagen, ermöglichen Prozesse der Anrufung im Sinne Louis Althussers (1977) - ein Sachverhalt, der unter dem Begriff der Subjektivierung diskutiert wird und als zentrales analytisches Moment der hier vorliegenden Arbeit in den Blick gerät: 
"Subjektivation bezeichnet den Prozeß des Unterworfenwerdens durch Macht und zugleich den Prozeß der Subjektwerdung. Ins Leben gerufen wird das Subjekt, sei es mittels Anrufung oder Interpellation im Sinne Althussers oder mittels diskursiver Produktivität im Sinne Foucaults, durch eine ursprüngliche Unterwerfung unter die Macht. Foucault weist zwar auf die Ambivalenz dieser Formulierung hin, geht jedoch nicht weiter auf die spezifischen Mechanismen der Subjektbildung in der Unterwerfung ein." (Butler 2001: 8)

Die Gleichzeitigkeit von Bemächtigung und Entmächtigung ist damit Gegenstand der vorliegenden Arbeit. Allerdings ist an dieser Stelle die Frage relevant, inwiefern Subjektivierung bzw. das Subjekt empirisch beobachtbar ist oder ob es sich vielmehr um eine »philosophisch-erkenntnistheoretische, keine naturale oder empirische Kategorie« (Gehring 2008: 21) handelt - ein zentraler Aspekt der vorliegenden Arbeit, der aufgegriffen wird. Sprechen über Jugendgewaltprävention schließt Sprechen über Jugend bzw. Jugendliche mit ein, was aus jugendsoziologischer Perspektive reflektiert wird: Aktuelle soziologische Literatur übt Kritik an einer Vereinseitigung des Sprechens über Kinder und Jugendliche und plädiert für eine Sichtweise, die Kinder und Jugendliche als soziale Akteure in den Blick nimmt (vgl. Bühler-Niederberger 2007, 2010, 2011; Breidenstein/Kelle 1998; Kelle 1996), was durch sozialpädagogische Praktiken, insbesondere auch durch präventive Maßnahmen, unter dem Stichwort Peer-Education aufgegriffen, jedoch wiederum stark problematisiert wird (vgl. Strauß 2012; Turner/Shepherd 1999). Die hier vorliegende Arbeit, die sich der Praxis des Sprechens über Jugend bzw. Jugendliche bedient, veranschaulicht Begrenzungen des Sprechens mit Jugendlichen im Kontext der Logik von Prävention, deren »Strategien mit der Auflösung des Begriffs des Subjekts oder des konkreten Individuums verbunden sind, der durch einen Komplex von Faktoren, die Risikofaktoren, ersetzt wird « (Castel 1983: 51). Inwiefern orientieren sich die Fachpersonen im Kontext von Jugendgewaltprävention, insbesondere der Sozialen Arbeit, an so beschaffenen Risikodiskursen?

\section{3 ÜBer das Verhältnis von Subjekt, Macht und Wissen}

Wie bereits einführend beschrieben, ist der Begriff des Subjekts in der vorliegenden Arbeit relevant, da hier das »Sprechen über« Jugendgewaltprävention analysiert wird, wobei den Adressierungsweisen und Subjektivierungsformen besondere Aufmerksamkeit geschenkt wird. Subjektivierungsprozesse stehen im Anschluss an Foucault immer mit Aspekten von Macht und Wissen in Zusammenhang. Diskurse werden als Erfahrungsräume betrachtet, die das »Sprechen-über-als« ermöglichen. Diesen Prozess beschreibt Foucault in seinen Studien über Sexualität (vgl. 1979 und 1986) als einen Prozess, der in 
Europa historisch an die Bildung der Nationalstaaten und damit der Bevölkerungskontrolle gekoppelt ist:

"Der Sex, das ist nicht nur eine Sache der Verurteilung, das ist eine Sache der Verwaltung. Er ist Sache der öffentlichen Gewalt, er erfordert Verwaltungsprozeduren, er muß analytischen Diskursen anvertraut werden. Der Sex wird im 18. Jahrhundert zu einer Angelegenheit der ,Polizei‘. [...] Polizei des Sexes: das ist nicht das strikte Verbot, sondern die Notwendigkeit, den Sex durch nützliche und öffentliche Diskurse zu regeln. [...] Durch die Politische Ökonomie der Bevölkerung hindurch bildet sich ein ganzes Raster von Beobachtungen über den Sex. [...] Der Staat muß wissen, wie es um den Sex der Bürger steht und welchen Gebrauch sie davon machen. Aber auch jeder einzelne muß fähig sein, den Gebrauch, den er vom Sex macht, zu kontrollieren." (Foucault 1979: 36f.)

Foucault befasst sich aus einer historischen Perspektive mit der Entstehung von Sexualität und ihrer Subjekte. Er beschreibt, wie Sexualität bereits im Prozess ihrer Entstehung normalisiert worden ist. Sein Ausgangspunkt ist die kritische Hinterfragung der, insbesondere von feministischen Bewegungen vertretenen, »Repressionshypothese «, nach der Frauen im 20. Jahrhundert sexuell befreit worden seien. Dieser Hypothese setzt er das Argument entgegen, dass das, was die »modernen« Menschen im 20. Jahrhundert im Allgemeinen unter Sexualität verstehen, v.a. Heteronormativität ist: Sexualität spielt sich zwischen Mann und Frau ab, innerhalb einer festen Partnerschaft bzw. Liebesbeziehung, folgt bestimmten Regeln, nimmt bestimmte Formen an und ist »privat«. Foucault hingegen zeigt, dass Sexualität nie Privatsache, sondern zunächst Gegenstand entstehender Institutionen der öffentlichen Verwaltung gewesen ist, der wiederum als Projektionsfläche für Individuen dient, die, auf ihn verweisend, sich selbst als im Staat agierende Akteure positionieren können. Das Wissen über Sexualität befähige Individuen dazu, Sexualität zu gebrauchen. Foucault zeichnet in seiner Analyse Prozesse der Etablierung von Sexualität als Diskursgegenstand nach, die Subjektivierungsprozesse und damit auch Identifikationsprozesse einschließen, welche die Erfahrung, die Selbstzuschreibung und schließlich die »Behandlung « von Sexualität ermöglichen: ${ }^{2}$

2 | Die diskursive Entstehung von Sexualität schließt die Entstehung ihrer Pathologien mit ein. "Die schulischen oder psychiatrischen Institutionen mit ihrer vielköpfigen Bevölkerung, ihrer Hierarchie, ihren räumlichen Anordnungen und ihrem Überwachungssystem bilden neben der Familie eine weitere Art und Weise, das Spiel der Mächte und Lüste zu organisieren; aber wie diese stecken auch sie Gebiete hoher sexueller Sättigung ab, mit privilegierten Räumen beziehungsweise Riten wie dem Klassenraum, dem Schlafsaal, der Visite oder der Konsultation. Hier werden die Formen einer nichtehelichen, nicht-heterosexuellen und nicht-monogamen Sexualität hervorgerufen und installiert. Die ıbürgerlicher Gesellschaft des 19. Jahrhunderts - zweifellos noch 
"Es ging mir also darum, zu sehen, wie sich in den modernen abendländischen Gesellschaften eine 'Erfahrung، konstituiert hat, die die Individuen dazu brachte, sich als Subjekte einer 'Sexualitätı anzuerkennen, und die in sehr verschiedene Erkenntnisbereiche mündet und sich an ein System von Regeln und Zwängen anschließt. Das Projekt war also das einer Geschichte der Sexualität als Erfahrung [...]." (Foucault 1986: 10)

In diesem Sinne ist das Subjekt zentraler Gegenstand von Foucaults Auseinandersetzungen mit modernen Gesellschaften und den diese prägenden Machtverhältnissen. Im Anschluss an Foucault beschäftigt sich Jaques Donzelot, ebenfalls aus historischer Perspektive, in »Die Ordnung der Familie« mit der Entstehung der Familie im 18. Jahrhundert (vgl. Donzelot 1980). Er zeigt auf, wie um die Familie herumkreisend, Institutionen und Strukturen entstanden bzw. sich festigten und in der Moderne als selbstverständlich etablierten, wie z.B. die Polizei, die Medizin, die staatliche Fürsorge, der Sozialwohnungsbau etc. Er verdeutlicht dabei, dass es sich bei dem Diskurs über das »gute Leben« primär um einen ökonomischen Diskurs handelte. Die Entstehung der staatlichen Fürsorge für junge Menschen hinsichtlich Gesundheit und Bildung war nicht altruistischer Motivation oder idealistischer Weltanschauung geschuldet, sondern zielte auf die Entwicklung und Aufrechterhaltung des Staatsapparats und darüber hinaus auf die Steigerung ökonomischer Leistungsfähigkeit ab. ${ }^{3}$ Aus seinen Quellenstudien bezüglich der hohen Kindersterblichkeit und der damit verbundenen Bemühungen um die Durchsetzung von Maßnahmen hinsichtlich der Unterbringung und Versorgung verwaister Neugeborener und Kinder sowie der Erziehung von Kindern aus adeligen Schichten schließt er wie folgt:

die unsere - ist eine Gesellschaft der blühendsten Perversion. [...] Nun besitzt diese Macht weder die Form des Gesetzes noch die Wirkungen des Verbots. Sie vollzieht sich stattdessen durch Vermehrung spezifischer Sexualitäten. Sie setzt der Sexualität keine Grenzen, sondern dehnt ihre verschiedenen Formen aus, indem sie sie auf unbegrenzten Durchdringungslinien verfolgt. Sie schließt sie nicht aus, sondern schließt sie als Spezifizierungsmerkmal der Individuen in den Körper ein. Sie sucht ihr nicht auszuweichen, sondern zieht mit Hilfe von Spiralen, in denen Macht und Lust sich verstärken, ihre Varietäten ans Licht; sie errichtet keine Blockade, sondern schafft Orte maximaler Sättigung. Sie produziert und fixiert die sexuelle Disparität. Die moderne Gesellschaft ist pervers, aber nicht trotz ihres Puritanismus oder als Folge ihrer Heuchelei; sie ist wirklich und direkt pervers." (Foucault 1979: 62f.)

3 | Foucault beobachtet eine ab dem 18. Jahrhundert zunehmende Milderung der Strafe, die er nicht auf zunehmende Menschlichkeit, sondern auf gesellschaftliche Interessen zurückführt: "Aber die Bestrafung soll nicht mehr nur im Spiel der Interessen der anderen, der Umgebung, der Gesellschaft usw. verankert werden. Ist die Bestrafung vorteilhaft, welchen Nutzen bietet sie, welche Form muß die Bestrafung haben, damit sie für die Gesellschaft von Vorteil ist?" (Foucault 2004b: 75f.) 
"Alle diese Abhandlungen bemühen sich demgegenüber zu zeigen, wie nützlich es wäre, die Bastarde am Leben zu erhalten, um sie nationalen Aufgaben wie der Kolonisation, der Miliz, der Marine zuzuführen. Aufgaben, für die sie sich vorzüglich eignen, weil sie frei von familiären Bindungen sind. [...] An einem Extrempunkt des gesellschaftlichen Körpers, dem der Armut, klagt man die Irrationalität der Heimverwaltung und den geringen Nutzen an, den der Staat aus der Aufzucht einer Bevölkerung zieht, die nur in seltenen Fällen ein Alter erreicht, in der sie die Unkosten erstatten könnte, die sie verursacht hat." (Donzelot 1980: 23ff.)

Er beobachtet, wie sich die »bürgerliche Gesellschaftsschicht« zwischen den beiden Extremen als Ort für die Etablierung einer »gesellschaftlichen Ökonomie«, einer »Ökonomie des Körpers« (ebd.: 26), und damit als Ort der diskursiven Etablierung eines Familienideals wiederfand, das den übrigen Gesellschaftsschichten zugänglich gemacht werden sollte. Als eine besondere Rolle sei dabei die der Hausfrau und Mutter entstanden, die durch ein Bündnis mit dem Hausarzt eine Aufwertung erfahren habe - ein Prozess, aus dem Pflegeberufe als »Profession der Frauen « und in diesem Zusammenhang die ersten Frauenbewegungen hervorgegangen seien. Die Aufwertung dieser Rolle sei jedoch gleichzeitig mit einer Abwertung einhergegangen, insofern der Machtgewinn bezüglich des »Privaten « - des häuslichen Bereichs - mit einem Machtverlust hinsichtlich des »öffentlichen Lebens« in Zusammenhang steht. Die Konzepte »Subjekt«, »Macht« und »Wissen« sind nach Foucault nicht voneinander zu trennen. Für die Erforschung des »Subjekts« im Anschluss an Foucault ist der Machtbegriff zentral:

"Nicht die Macht, sondern das Subjekt ist deshalb das allgemeine Thema meiner Forschung. Aber die Analyse der Macht ist selbstverständlich unumgänglich. Denn wenn das menschliche Subjekt innerhalb von Produktions- und Sinnverhältnissen steht, dann steht es zugleich auch in sehr komplexen Machtverhältnissen." (Foucault 1994b: 243)

Die Ermöglichung des Sprechens über Sexualität, Krankheit, professionelle (Selbst-)Verständnisse etc. setzt das Wissen über diese Konstrukte voraus und ist nicht als bloße Entmächtigung durch Interessen nationalstaatlicher Institutionen zu begreifen, sondern gleichzeitig als Bemächtigung:

"Diese Form von Macht wird im unmittelbaren Alltagsleben spürbar, welche das Individuum in Kategorien einteilt, inm seine Individualität aufprägt, es an seine Identität fesselt, ihm ein Gesetz der Wahrheit auferlegt, das es anerkennen muß und das andere in ihm anerkennen müssen. Es ist eine Machtform, die aus Individuen Subjekte macht. Das Wort Subjekt hat einen zweifachen Sinn: vermittels Kontrolle und Abhängigkeit jemandem unterworfen sein und durch Bewußtsein und Selbsterkenntnis seiner eige- 
nen Identität verhaftet sein. Beide Bedeutungen unterstellen eine Form von Macht, die einen unterwirft und zu jemandes Subjekt macht." (Ebd.: 246f.)

Nach Foucault setzt Macht folglich Freiheit voraus und drückt sich nicht allein im Zwang aus (vgl. Bröckling/Krasmann 2010; Foucault 1994; Foucault/ Defert 2005). Mit der Distanzierung von einem negativen Konzept von Macht rückt eine Beschreibung von Macht als positive und produktive Kraft in den Vordergrund (vgl. Han 2010: 44f.). In diesem Sinne ist Macht als dialektisches Verhältnis von Bemächtigung und Entmächtigung zu verstehen, wie Giorgo Agamben auch bezüglich der Wirkungsweisen von Dispositiven erkennt:

"So konnte Foucault zeigen, wie in einer Disziplinargesellschaft die Dispositive mittels einer Reihe von Praktiken und Diskursen, Kenntnissen und Übungen auf die Schaffung gelehriger, doch freier Körper zielen, die ihre Identität und ihre 'Freiheit، in eben dem Prozeß ihrer Unterwerfung erlangen. Das Dispositiv ist zunächst also eine Maschine, die Subjektivierungen produziert, und nur als solche ist es auch eine Regierungsmaschine." (Agamben 2008: 35)

Als »Regierungsmaschine« lässt sich nun auch der Diskurs über Jugendgewaltprävention beschreiben. Fachpersonen, die in ihrer Verantwortung für Jugendgewaltprävention adressiert sind, ist es ermöglicht, als »Expertin« bzw. »Experte« über Jugendgewaltprävention zu sprechen. Dies ist gerahmt von einem auf kriminalpolitischer Ebene angesiedelten Programm, das bestimmten Personen und Personengruppen Rollen der Zuständigkeit zuweist:

"Das gesamtschweizerische Präventionsprogramm Jugend und Gewalt richtet sich an die für Gewaltprävention verantwortlichen Fachpersonen und Entscheidungsträgerinnen auf kantonaler und kommunaler Ebene. Gewaltprävention wird vor Ort nicht nur von kantonalen und kommunalen Behörden betrieben. Zahlreiche Nichtregierungsorganisationen, Vereinigungen und Private arbeiten teilweise schon seit vielen Jahren Konzepte und Projekte zur Gewaltprävention aus. Diese Akteure sind für eine erfolgreiche Prävention von Jugendgewalt ebenso wichtig. Ihre Erfahrungen werden im Rahmen von Expertengruppen, Konferenzen und thematischen Seminaren berücksichtigt. Zudem können sie ihre Angebote über die Kantone und Gemeinden als Pilotprojekt vorschlagen oder für eine wissenschaftliche Evaluation einreichen. " ${ }^{4}$

Aus dem »Fact Sheet « des »Nationalen Präventionsprogramms Jugend und Gewalt« wird ersichtlich, wie weit die Adressierung von Verantwortung reicht, wobei das Monopol bei Kantonen und Kommunen liegt. Dies gewinnt ange-

4 | Das "Fact Sheet" des Programms finden Sie online unter www.news.admin.ch/NSBSubscriber/message/attachments/34322.pdf vom 12.01.2017. 
sichts des Ziels des Programms, über verschiedenste Strategien der Vernetzung, Standardisierung und Implementierung sog. »evidenzbasierter« Projekte ein gemeinsames Wissen bezüglich der »Prävention und Bekämpfung von Jugendgewalt« zu bündeln, an Plausibilität. So stellt die vorliegende Arbeit die Frage, welche Anschlussmöglichkeiten sich für Fachpersonen unterschiedlicher Bereiche im Rahmen der durch das »Nationale Programm Jugend und Gewalt« formulierten Adressierungen und Forderungen ergeben.

\subsection{SPPReChen Über GeWALt: ÜBer das Verhältnis von Sprechen und Gewalt}

Im Folgenden wird die Bedeutung einer Unterscheidung zwischen Gewalt und Sprechen über Gewalt herausgestellt, woraus methodologische und methodische Implikationen folgen. Gegenstand der hier vorliegenden Arbeit ist das Sprechen über Jugendgewalt bzw. Jugendgewaltprävention. Gewalt tritt also als diskursiver Gegenstand in den Blick und nicht als Phänomen oder Praktik. Sprechen über Gewalt wird diskursanalytisch und somit machtanalytisch untersucht. Der Sprechakt selbst wird aber nicht als Gewalt konzeptualisiert, wie es etwa Judith Butler in ihren Überlegungen zur »sprachlichen Verletzbarkeit« tut (Butler 2006: 14). Dem Unterschied zwischen Macht und Gewalt wird in aktuellen soziologischen und philosophischen Diskussionen hohe Bedeutung zugemessen, wie folgend gezeigt wird. Eine diskurstheoretische Herangehensweise an Jugendgewaltprävention kann vor dem Hintergrund der Beobachtung und Kritik eines in den Sozialwissenschaften und in der sozialpädagogischen Praxis dominierenden »weiten Begriffs von Gewalt« (Felten 2000: 33) sowie seiner vielseitigen Verwendung als »Skandalisierungsformel« (Liell 1999: 40) oder auch »Skandalisierungsfalle« (Cremer-Schäfer 1998: 132) und seiner Skandalisierung auf verschiedensten gesellschaftlichen Bühnen (vgl. Krasmann 1997; Krasmann/Scheerer 1997; Liell 1999; 2002; Stehr 2009) aufschlussreich sein, da er das »Wesen« von Gewalt diskursiv überlagert und folglich die Bedeutung »realer « Gewalt dahinter zu verschwinden droht (vgl. Krasmann 1997; Krasmann/Scheerer 1997). Ist der »Inhalt« von Gewalt überhaupt sprachlich fassbar? Auf theoretischer Ebene gelingt es Byung-Chul Han, so etwas wie eine »Substanz« von Gewalt in Abgrenzung zum Machtbegriff herauszuschälen (vgl. Han 2011), was anschlussfähig für eine Kritik eines »weiten« Begriffs von Gewalt ist, die an der Gefahr der Nivellierung (vgl. Felten 2000) ansetzt. Auch Han selbst plädiert für eine begriffliche Schärfung des Gewaltbegriffs, insbesondere aus einer Kritik Johan Galtungs Theorie der strukturellen Gewalt (1975) heraus: 
"Auch Galtungs Theorie der sstrukturellen Gewalt liegt die Annahme einer strukturellen Vermitteltheit der Gewalt zugrunde. [...] Dieser Gewaltbegriff ist zu allgemein, so dass er gerade nicht das erfasst, was die Gewalt eigentlich ausmacht, d.h. sie von anderen negativen gesellschaftlichen Zuständen unterscheidet. Dass Kinder der Arbeiterklasse weniger Bildungschancen haben als Kinder der Oberschicht, ist noch keine Gewalt, sondern eine Ungerechtigkeit. Wird die Gewalt zum Chiffre für die allgemeine gesellschaftliche Negativität entgrenzt, so verschwimmt ihr Begriffsprofil ganz." (Han 2011: 101f.)

Einer positiven Beschreibung von Macht als produktive Kraft, die etwas hervorbringt, steht eine negative Beschreibung von Gewalt, die nur zerstört, gegenüber. Han beschreibt weiter einen »topologischen Wandel der Gewalt« (ebd.: 9) von einer negativen in eine positive Form und setzt Gewalt und Macht damit zwar in (eine historische und gesellschaftliche) Beziehung zueinander, ohne jedoch verschiedene Formen von Gewalt unter Macht zu subsummieren. Gleichzeitig bettet er sie als Ausdrucksformen von Gewalt in ihren gesellschaftlichen Kontext ein. Während negative Formen von Gewalt sich auf »den oder das andere beziehen« - als Phänomen also zwischen Alter und Ego situiert sind und als solche, historisch gesehen, tabuisiert worden sind -, benötigen positive Formen von Gewalt den Bezug auf das »Fremde« nicht mehr, sondern beziehen sich auf das »Selbst«. Gewalt verschwindet historisch gesehen nicht, sondern verändert ihre Ausdrucksform zwischen zwei Polen entlang gesellschaftlicher Veränderungen. Positive Gewalt schreibt Han dem »spätmodernen Leistungssubjekt« zu,

"welches insofern frei ist, als es keiner Repression durch eine inm äußere Herrschaftsinstanz ausgesetzt ist. In Wirklichkeit ist es aber genauso unfrei wie das Gehorsamssubjekt. Wird die äußere Repression überwunden, entsteht die Pression im Inneren. [...] Die Gewalt wird zunehmend internalisiert, psychisiert und dadurch invisibilisiert." (Ebd.: 9)

In diesem Zusammenhang beschreibt Han Depression als positive Form von Gewalt, die moderne Gesellschaften prägt und im Gegensatz zu negativen Formen von Gewalt weniger tabuisiert ist bzw. zunehmend enttabuisiert wird. Die hier vorliegende Arbeit hat nicht den Anspruch, die Unterscheidung zwischen Macht und Gewalt bzw. verschiedenen Formen von Gewalt phänomenologisch zu erfassen (vgl. Staudigl 2007), jedoch distanziert sie sich auch von den »klassischen« soziologischen Theorien über Gewalt, die, Hand in Hand mit der Kriminologie, auf eine »Jagd nach Ursachen« (ebd.: 236) gehen. Stattdessen reflektiert sie über die Möglichkeit des Sprechens über Gewalt im Kontext von Jugendgewaltprävention, rekonstruiert Jugendgewalt dabei als Deutungsmuster und weist schließlich darauf hin, wie die Fachpersonen ihr Handeln in Bezug auf Jugendgewaltprävention legitimieren. Mit der Annahme, Deutungen und 
Wahrnehmungen von Gewalt hätten handlungspraktische Konsequenzen, wird theoretisch ein Bogen geschlagen, der Gewalt auf Deutungsebene und auf phänomenologischer Ebene denken lässt und demnach den »Doppelcharakter von Gewalt« (Liell 1999: 45) ernst nimmt, ohne das »reale Phänomen« zu verharmlosen. Um mit Susanne Krasmann zu sprechen, geht es in der vorliegenden Arbeit darum, die Fragen, »inwieweit Diskurse die Wahrnehmung von Gewalt als Problem strukturieren? Und wie wird umgekehrt das Erkennen von Gewalt als soziales Problem durch entsprechendes Sprechen darüber ausgeschlossen oder verhindert? « (Krasmann 1997: 97), in den Kontext von Jugendgewaltprävention zu setzen und zu erschließen, welche (Un-)Möglichkeiten des Sprechens über Jugendgewalt diesen Kontext prägen. Jugendgewalt, so die These, wird im Rekurs auf kriminalpolitische Programmatik als Deutungsmuster reproduziert und fungiert somit als zentrales Moment gesellschaftlicher Ordnungsbildung. So gesehen kann Gewalt als eine Leerformel verstanden werden, wie übrigens auch Prävention (vgl. Widersprüche 2001: 3). Das Fassungsvermögen der Begriffe »Gewalt« und »Prävention« erscheint unbegrenzt und gleichzeitig von enormer Bedeutsamkeit, wodurch eine Explikation behindert und möglicherweise nicht als notwendig erachtet wird: Jeder versteht sofort, was damit gemeint ist, und versteht, dass Gewalt schlecht und Prävention gut ist. Trotzdem ist im Fall unklar, über was eigentlich tatsächlich gesprochen wird und was das macht. Dies ist Gegenstand der hier vorliegenden Arbeit. 


\section{Methodische Herangehensweise}

\subsection{Zur VerknüPfung von Diskursanalyse UNd DokUmentarischer Methode}

Die vorliegenden Interpretationen erfolgen mithilfe der dokumentarischen Methode im Anschluss an Karl Mannheim (vgl. Bohnsack/Nentwig-Gesemann/Nohl 2001; Mannheim 1980; Nohl 2006). Die Stichprobe umfasst neun offene leitfadengestützte Interviews mit Fachpersonen, die im Bereich der Jugendgewaltprävention tätig sind. Im Kontext des »Nationalen Präventionsprogramms Jugend und Gewalt« werden die verschiedensten Fachbereiche als Verantwortliche adressiert. Verantwortung wird nicht nur Sozialer Arbeit, insbesondere der Jugend- und Sozialarbeit (offene, aufsuchende, schulische), zugeschrieben, sondern auch Familientherapie, Polizei, privaten Organisationen sowie Institutionen juristischer und medizinischer Couleur. ${ }^{1}$ Im Rahmen dieser Arbeit werden Interviews mit Fachpersonen verschiedener Fachbereiche hinsichtlich ihrer Gemeinsamkeiten (und Unterschiede) in Bezug auf ihre Orientierungsweisen an kriminalpolitischer Programmatik untersucht. Damit wird auch der Heterogenität des Forschungsgegenstands Rechnung getragen, insoweit er theoretisch als »Diskurs« relevant wird, empirisch sich jedoch als Zusammenhang verschiedener Diskursfragmente darstellt. »Der Diskurs« über Jugendgewaltprävention zeigt sich verwoben mit aktivierungspolitischen Diskursen (vgl. Dollinger 2006; Kocyba 2009) sowie Risiko- und Sicherheitsdiskursen (vgl. Berner/Groenemeyer 2003; Groenemeyer 2015). ${ }^{2}$ Daraus erge-

1 Der Zusammenhalt zwischen den verschiedenen Fachbereichen und die Bedeutung des Schaffens einer gemeinsamen Wissensbasis ist dabei eines der zentralen, immer wieder reformulierten Anliegen des Programms (vgl. Kapitel 4.2).

2 | Berner und Groenemeyer unterscheiden zwischen "drei unterschiedlichen Teildiskursen “ in Bezug auf den "Diskurs der ,kommunalen Kriminalprävention، [...]: (1) ein ,Sicherheitsdiskurs als Probleminterpretation und handlungsmotivierende Wertidee, (2) ein 'Gemeinschaftsdiskursı als ebenfalls wertgeladenes Leitbild für Problemlösungen und (3) ein 'Zuständigkeitsdiskursı mit handlungsleitenden Problemerklärungen und Zuständigkeitsdefinitionen." (2003: 90) 
ben sich methodologische und methodische Herausforderungen in Bezug auf die analytische Eingrenzung des zu beforschenden empirischen Gegenstands, die im Kapitel 4.1 reflektiert werden.

"Was den Diskurs als Gegenstand zu einer besonderen methodologischen Herausforderung für die Diskursanalyse macht, ist, dass er sich aus verschiedenen Komponenten zusammensetzt und in seiner heterogenen Beschaffenheit betrachtet werden muss. [...] Die Frage ist vielmehr, wie dem sozialen Gebrauch Rechnung getragen wird, den bestimmte Teilnehmerlnnen eines Diskurses zu einer bestimmten Zeit an einem bestimmten Ort von der Sprache und anderen semiotischen Ressourcen machen, um Sinn zu produzieren." (Angermüller 2014: 25)

Gegenstand der Analyse ist in diesem Sinne das Sprechen über Jugendgewaltprävention »zu einer bestimmten Zeit an einem bestimmten Ort« und der diesem Sprechen immanente Gebrauch diskursiven Wissens. Die sich aus diesem diskursspezifischen Zusammenhang sowie der Erforschung von Diskursen im Allgemeinen ergebende methodische Grundfrage richtet sich an die Transformation diskursspezifischer Wissensbestände, an denen sich die Fachpersonen in der Reflexion ihres beruflichen Alltagshandelns orientieren, in die je spezifischen organisatorischen bzw. institutionellen Felder. ${ }^{34}$ Die Gemeinsam-

3 | Ralf Bohnsack zur Sinn- bzw. Soziogenese als Verfahren der dokumentarischen Interpretation: "Die Identifikation dieses generativen Musters, also dessen Interpretation, setzt die Beobachtung einer Handlungspraxis voraus. Diese kann uns entweder unmittelbar gegeben sein oder auf dem Wege von Erzählungen und Beschreibungen der Erforschten. Das generative (Sinn-)Muster bezeichnen wir - wie gesagt - als Orientierungsrahmen oder auch als Habitus." (Bohnsack 2001: 231) Mittels der Dokumentarischen Methode für Interviews wird in der hier vorliegenden Arbeit das den Fachpersonen gemeinsame "Subjekt-Wissen « rekonstruiert, was Hinweise auf die Wirkmächtigkeit kriminalpolitischer Programmatik gibt - im Sinne ihrer Relevanz für das Deuten und Handeln im Kontext der je spezifischen Fachbereiche.

4 | Die im Rahmen diskursanalytischer Ansätze zu untersuchende Wirkmächtigkeit von Diskursen (vgl. Schwab-Trapp 2010: 178f.) wird auch auf organisatorischer bzw. institutioneller Ebene sog. "Expertinnen“ bzw. Experten zugeschrieben: "Im theoriegenerierenden Experteninterview befragen wir Experten, weil ihre Handlungsorientierungen, ihr Wissen und ihre Einschätzungen die Handlungsbedingungen anderer Akteure in entscheidender Weise (mit-)strukturieren und damit das Expertenwissen die Dimension sozialer Relevanz aufweist. [...] Demnach lassen sich Experten als Personen verstehen, die sich - ausgehend von spezifischen Praxis- oder Erfahrungswissen, das sich auf einen klar begrenzbaren Problemkreis bezieht - die Möglichkeit geschaffen haben, mit ihren Deutungen das konkrete Handlungsfeld sinnhaft und handlungsleitend zu strukturieren." (Bogner/Menz 2009: 72, 73) 
keit der Fachpersonen liegt vornehmlich darin, dass sie Adressatinnen und Adressaten kriminalpolitischer Programmatik sind. In der vorliegenden Arbeit wird untersucht, inwiefern sie sich an dieser Programmatik in der Beschreibung ihres beruflichen Alltagshandelns orientieren, beispielsweise bezüglich ihrer Devianzkonstruktionen und Präventionskonzepte. Diese Orientierungsweisen können, diskursanalytisch gesprochen, auf Subjektivierungsformen zurückgeführt werden:

"Die diskursiv vermittelten Subjektformierungen und -positionierungen enthalten Wissen darüber, wer der einzelne im Verhältnis zu den anderen sein soll, welche Praktiken dabei zu verfolgen sind und welche Bewertungen damit einherzugehen haben. Dabei ist nicht nur dieses diskursiv vermittelte Subjekt-Wissen empirisch zu rekonstruieren, sondern auch empirisch zu klären, was von alledem von Individuen wie (über welche SelbstPraktiken) angeeignet und in Alltagshandeln umgesetzt wird." (Bührmann/Schneider 2008: 69)

Eine der methodischen Herausforderungen der Arbeit, welche die Orientierungsweisen der Fachpersonen im Kontext von Jugendgewaltprävention an kriminalpolitischer Programmatik aus diskurstheoretischer Perspektive beobachtet, liegt nun darin, dass theoretisch davon auszugehen ist, dass die »diskursiv vermittelten Subjektformierungen« in der sozialen Praxis des Interviews reproduziert werden, was methodisch reflektiert werden muss, damit Aussagen über die Rekonstruierbarkeit handlungsrelevanter Orientierungsrahmen und Deutungsmuster getroffen werden können. Dabei greift die vorliegende Arbeit auf Literatur zu »Experteninterviews« zurück, da diese die Interviews als Interaktionen begreift und dabei Adressierungsweisen thematisiert (vgl. Bogner/Menz 2009). Außerdem nimmt sie Bezug auf Literatur, die methodische Herausforderungen diskursanalytischer Arbeiten fokussiert, insbesondere die Integration interpretativer Methoden (vgl. Angermüller/Nonhoff/Herschinger 2014). Aus dieser Perspektive fungiert das Interview als Diskurs(-re-) produktion, an der die Forscherin selbst beteiligt ist, was sie in ihre Analyse miteinbeziehen muss. »Mit Blick auf die ethische Haltung kann die Methode der Diskursanalyse als ein Element gesellschaftlicher Veränderung betrachtet werden, insofern die die Diskurse Analysierenden ihre diskursanalytische Tätigkeit selbst als Diskursproduktion reflektieren.« (Bublitz et al. 1999: 14) Diese Betrachtung gilt für Forschungsarbeiten, die in diesem Kontext Interviews generieren, was nicht als Praxis »klassischer« wissenssoziologischer Diskursanalyse angesehen wird.

"Die Wissenssoziologische Diskursforschung begreift Texte, Praktiken oder Artefakte nicht als Produkte 'subjektiver oder robjektiver Fallstrukturen, sondern als materiale Manifestationen gesellschaftlicher Wissensordnungen und damit als wichtigste Grund- 
lage einer wissenssoziologischen Rekonstruktion der Produktion, Stabilisierung und Veränderung kollektiver Wissensvorräte." (Keller 2004: 78)

Für das Forschungsvorhaben der vorliegenden Arbeit, die Orientierungsweisen der Fachpersonen im Kontext von Jugendgewaltprävention an kriminalpolitischer Programmatik fokussiert, lässt sich also folgern, dass eine Trennung von »Diskurs « und »Praxis« nur als eine analytische fungieren kann und Interviews als diskursive Praktiken zu begreifen sind:

"Die erste Anforderung lautet, Praktiken und Diskurse nicht als zwei unabhängige Gegenstände zu separieren - von denen dann, je nach theoretischem Hintergrund, jeweils einem eine lediglich sekundäre Bedeutung zukommt -, sondern als zwei aneinander gekoppelte Aggregatzustände der materialen Existenz von kulturellen Wissensordnungen zu begreifen." (Reckwitz 2008b: 201)

Insofern haben die Trennung verschiedener Datenkorpora wie auch der Subjektbegriff primär analytischen Charakter. Im Anschluss an Foucault werden Subjektivierungsprozesse hier in ihrer Gleichzeitigkeit von Bemächtigung und Entmächtigung gedacht, insofern sich die Subjekte in ihrem Gebrauch diskursiv erzeugten Wissens gleichzeitig in einer Weise bemächtigt und in anderer Weise entmächtigt ansehen können. ${ }^{5}$ Die vorliegende Arbeit zeigt auf, inwiefern sich die Fachpersonen in der Beschreibung ihrer »Präventionsarbeit« an diskursiv erzeugten Wissensbeständen orientieren, auf welche Deutungen von Jugendgewalt sie dabei rekurrieren und welche Deutungen von Prävention sie vor diesem Hintergrund rekonstruieren. Dabei wird auch in den Blick genommen, auf welche Weise Jugendliche auf »programmatischer Ebene« und »Praxisebene « adressiert werden. ${ }^{6}$ Die Literatur charakterisiert Prävention als »Antizipation von Gefahren, Risiken, Unsicherheiten«, die

5 | Der Terminus "Subjekt" referiert auf Foucaults Forschungen über die Entstehung von Heterosexualität als normative Matrix: "Schließlich habe ich versucht, die Art und Weise, in der ein Mensch sich selber in ein Subjekt verwandelt, zu untersuchen. Als Beispiel habe ich den Bereich der Sexualität gewählt: wie der Mensch gelernt hat, sich als Subjekt einer 'Sexualität، zu erkennen." (Foucault 1994b: 244; vgl. auch Foucault 1979) 6 | Der Deutungsebene aus der Perspektive Jugendlicher kann im Rahmen der vorliegenden Arbeit aus folgenden Gründen nicht Rechnung getragen werden: Zum einen ist das hier untersuchte Ensemble an Institutionen und Organisationen zu heterogen, zum anderen fokussiert die vorliegende Arbeit Transformationsprozesse zwischen der kriminalpolitischen Ebene und der Ebene sozialer Praxis und damit das Sprechen über Jugendliche, was sich in der Interviewpraxis manifestiert. Eine Anschlussarbeit, die sich im Rahmen einer Diskursethnografie (vgl. Keller 2011) auf eine oder wenige Institutionen bzw. Organisationen fokussiert, wäre erstrebenswert. 
"das Handeln in der Gegenwart beeinflusst - Prävention ist eine Weise der Regierung über die Zukunft. Dabei hängt die Wahrnehmung dessen, was es in welcher Weise zu verhindern gilt, wesentlich davon ab, welche Erwartungen und welches Wissen ihr zugrunde liegen. [...] Beruhen Präventionsmaßnahmen insofern nicht nur notwendig auf Interpretationen, sondern auch auf Imaginationen, so ist die entscheidende politische Frage nicht, ob diese real oder irreal sind, sondern welche Konsequenzen sie zeitigen." (Krasmann 2011: 53)

Insofern Prävention Kontingenz reduziert, indem sie bestimmte »Zukünfte« anvisiert und andere Zukunftsentwürfe ausblendet, kann sie als »repressiv« angesehen werden (vgl. Cremer-Schäfer 2016: 13). Die diskursiv vermittelten Wissensbestände und damit verbundenen Problematisierungsweisen und Präventionsverständnisse, an denen sich die Fachpersonen in der Reflexion ihres Arbeitsalltags orientieren, sind insofern für sie relevant, als davon ausgegangen werden kann, dass sie sich primär in Situationen der Abwesenheit von »Gefahren, Risiken und Unsicherheiten« befinden. Es ist davon auszugehen, dass sich die Fachpersonen in Situationen befinden, in denen Gewalt abwesend ist, insoweit die visuelle und kognitive Abwesenheit bzw. das Fehlen von sinnlich erfahrbarer Jugendgewalt in den jeweiligen organisatorischen und institutionellen Settings der Normalfall ist. Nach Randall Collins ist bezüglich der phänomenologischen Ebene im Gegensatz zur diskursiven Ebene davon auszugehen, dass

"Gewalt eine seltene und relativ unwahrscheinliche Form menschlichen Handelns darstellt. Denn es gibt nicht nur starke normative, sondern auch emotionale Barrieren, die überwunden werden müssen, um sie in Gang zu setzen. Dies gilt, betont der Autor [Collins], selbst für Gegenden oder Personen, die gemeinhin als extrem gewalttätig gelten. [...] Anders als es populäre Diskurse bisweilen suggerieren, stellt Gewalthandeln deshalb immer eine Ausnahme und nicht die Regel menschlicher Interaktion dar." (Koloma Beck/Schlichte 2014: 142f.; vgl. auch Collins 2011: 11)

Die Aneignung eines Präventionsauftrags, so die These dieser Arbeit, macht Imaginationen von Jugendgewalt notwendig, um je spezifisches Handeln zu legitimieren. In dieser Hinsicht fungiert Jugendgewalt in diesem Kontext als Deutungsmuster, das als »vermittelndes Glied zwischen objektiven gesellschaftlichen Handlungsproblemen und deren subjektiver Bewältigung « dient und »als eine sozialem Handeln zugrundeliegende, genauer: soziales Handeln erzeugende Regelstruktur« begriffen werden kann, »mit deren Hilfe Akteure ihren Alltag deuten, ordnen, organisieren bzw. ihre Deutungs- und Hand- 
lungsprobleme lösen« (Lüders/Meuser 1997: 58f.).7 Ziel der Analyse ist folglich die Rekonstruktion von Deutungsmustern von Jugendgewalt und daran anschließenden (Un-)Möglichkeiten von Jugendgewaltprävention als Praktik, insofern die

"in den Diskursen produzierten Deutungen als strukturierte, strukturierende und typisierte Formen organisiert sind bzw. als solche rekonstruiert werden können. Versteht man Diskursanalyse als eine Analyse sozialer Prozesse der Herstellung von Bedeutung, so bedeutet dies auch eine Analyse latenter Sinnstrukturen und damit zusammenhängender Regeln, die über die von innen angebotenen Deutungsmuster zur Konstruktion der (alltäglichen) Wirklichkeiten beitragen." (Althoff 2002a: 72)

Die hier beschriebenen Konstruktionsprozesse in der Situation des Sprechens über den »normalen Präventionsalltag« bzw. die »Präventionsarbeit« werden anhand von Datenbeispielen veranschaulicht, wobei die Interviewpraxis aus diskursanalytischer Perspektive reflektiert wird. Die folgenden Interpretationen erfolgen anhand methodischer Vergleiche von Fällen, die im Sinn der Grounded Theory aufgrund ihrer kontextuellen Unterschiedlichkeiten zumeist als »weithergeholte Vergleiche« (Strauss/Corbin/Niewiarra 1996: 69f.) fungieren. ${ }^{8}$ Dieses Vorgehen ähnelt der im Rahmen der dokumentarischen

7 | Das Konzept des Deutungsmusters fungiert zugleich als analytisches und empirisches Konzept, dem sich die vorliegende Arbeit mit der Methode des Vergleichs annähert. Die Arbeit bezieht sich hierbei einerseits auf die komparative Analyse im Rahmen der bereits erwähnten dokumentarischen Methode im Anschluss an Karl Mannheim, die "abhängig vom Standort des Interpreten " ist. "Sie wird umso mehr methodisch kontrollierbar, je mehr die Vergleichshorizonte des Interpreten empirisch fundiert und somit intersubjektiv nachvollziehbar und überprüfbar sind. [...] Die Erhöhung der Validität einer Fallanalyse ist also nicht nur an die zunehmende empirische Fundierung des jeweiligen Falles selbst, sondern auch an die zunehmende empirische Fundierung der Vergleichshorizonte gebunden, indem an die Stelle gedankenexperimenteller Vergleichshorizonte empirische, also andere empirische Fallanalysen treten." (Bohnsack 2003: 137) Andererseits bezieht sich die Arbeit auf die Grounded Theory, die den Abgleich von Theorie und Empirie sowohl als Analysemethode expliziert (vgl. Strauss/Corbin/Niewiarra: 66f.), als auch gezielt als Instrument der Validierung einsetzt (vgl. Glaser/Strauss 1979: 96).

8 | Die Auswahl der Interviewpassagen ist genau genommen bereits Teil der Interpretationsleistung der dokumentarischen Methode, die dazu anregt, nach thematisch vergleichbaren Interviewpassagen zu suchen: „Im Zuge der Abstraktion der rekonstruierten Orientierungsfigur, die dem Prinzip der Abduktion folgt, wird nun zuerst in thematisch vergleichbaren, also auf die familiale Interaktion bezogenen, Passagen aus Diskussionen mit anderen Gruppen nach einem analogen oder homologen Muster gesucht, 
Interpretation praktizierten »reflektierenden Interpretation«, der eine »formulierende Interpretation« vorausgeht. Die formulierende Interpretation entspricht einer zusammenfassenden Interpretation, wobei

"der Interpret innerhalb des (Orientierungs-)Rahmens der Gruppe bleibt, er macht diesen noch nicht zum Gegenstand begrifflich-theoretischer Explikation, sondern lediglich die angesprochenen Themen, indem er zusammenfassende 'Formulierungen . [...] im Sinne von Oberbegriffen, Überschriften oder Themen sucht und auf diese Weise eine Übersicht über den Text gewinnt. Es handelt sich um eine Interpretation`, da ja hier etwas begrifflich-theoretisch expliziert wird, was im Text implizit bleibt." (Bohnsack 2003: 134)

Erst in einem zweiten Interpretationsschritt, dem der reflektierenden Interpretation, wird der Interpretationsrahmen der Befragten mithilfe des methodischen Vergleichs reflektiert: ${ }^{9}$

welches dort möglicherweise in ganz anderen Formulierungen zum Ausdruck gebracht wird. [...] Eine derartige fallübergreifende komparative Analyse, mit der die Abstraktionsfähigkeit von Orientierungsmustern ausgelotet wird, sollte - sofern eine Typenbildung überhaupt angestrebt wird - schon sehr früh im Forschungsprozess erfolgen, weil auf diese Weise das Verallgemeinerungspotential von der fallspezifischen Besonderheit abgehoben werden kann. Die fallübergreifende komparative Analyse erfolgt also idealerweise soweit möglich bereits vorab der fallinternen komparativen Analyse, also der Abstraktion innerhalb eines Falles, die ich als Spezifizierung eines Typus bezeichne." (Bohnsack 2001: 234f.; vgl. auch Bohnsack 2003: 36) Auch andere, nicht primär sequenziell vorgehende, Interpretationsansätze empfehlen zu Beginn der Analyse thematische Gliederungen sowie zusammenfassende Interpretationen, was z.T. der Erhebungsmethode durch leitfadengestützte Interviews geschuldet ist (vgl. Meuser 1989: 83; Meuser/Nagel 2009a: 56, 2009b: 85f.; Wiedemann 1989: 214). Es empfiehlt sich hierbei - bereits vor der Transkription -, einen thematischen Ablauf der Interviews zu erstellen. Der diskursanalytische Zugang zur Interviewpraxis macht allerdings auch eine Analyse derselben notwendig, weshalb die Interviews hier zunächst in ihrer Fallspezifik und schließlich themenbezogen untersucht werden.

9 | In der "fallübergreifenden komparativen Analyse" werden Interviewsequenzen mit besonders "hoher narrativer Dichte, also einem hohen Detaillierungsgrad" (Bohnsack 2001: 233), vergleichend interpretiert, wobei kontrastierend vorgegangen wird. Es werden beispielsweise Passagen ausgesucht, in denen Erzählungen über die Relevanz von problematischen Situationen, wie etwa Situationen der Gewalt, Aufschluss geben. Im Anschluss an die Erzähltheorie von Fritz Schütze geht Hans-Christoph Koller in der Analyse biografischer Interviews ähnlich "wissensanalytisch und fallvergleichend" vor: "Mit dem wissensanalytischen Vergleich ist die Auswertung abgeschlossen, soweit es sich um die Interpretation eines einzelnen Interviewtextes handelt. Werden mehrere In- 
"Während die formulierende Interpretation als Rekonstruktion des Themas des Diskurses mit seinen Untergliederungen, also als Rekonstruktion der thematischen Gliederung zu verstehen ist, zielt die reflektierende Interpretation auf die Rekonstruktion und Explikation des Rahmens, innerhalb dessen das Thema abgehandelt wird, auf die Art und Weise, wie, d.h. mit Bezug auf welches Orientierungsmuster, welchen Orientierungsrahmen das Thema behandelt wird. Dort, wo die (theoretisierenden oder beschreibenden oder erzählenden) Darstellungen derartige Orientierungsmuster oder (Orientierungs-) Rahmen zum Ausdruck gebracht werden, spreche ich von ,Propositionen. Der Rahmen ist zunächst durch die Gegenhorizonte identifizierbar, innerhalb dessen das Thema abgehandelt wird." (Ebd.: 135f.)

Ergebnis dieses zweigeteilten Interpretationsprozesses sind zwei unterschiedliche Wissensformen - das »kommunikative Wissen « und das »konjunktive Wissen« (Nohl 2006: 43), wobei letzteres dem interessierenden Deutungs- bzw. Sinnmuster entspricht, an dem sich, wovon ausgegangen wird, die befragten Fachpersonen in ihrem Handeln orientieren, das aber

"nicht mehr mit jenem von dem oder den Produzenten intendierten Sinngehalt identisch ist, damit aber lediglich vom 'Rezeptiven ‘ her erfasst, vom Rezeptiven her konstruiert wird und somit in besonderer Weise von der Perspektive, vom 'Standort des Interpreten ، abhängig ist. Es ist also - methodisch-formal gewendet - abhängig von den Gegen- oder Vergleichshorizonten des Interpreten." (Bohnsack 2003: 42) ${ }^{10}$

Insofern sich die »Ebene des konjunktiven Wissens, als überindividuelles, gemeinschaftliches, habituelles Wissen, einerseits von individuellen Absichten, Vorstellungen und Meinungen der Akteure, andererseits von abstraktem, theoretischem Wissen abheben lässt« (Gaffer/Liell 2001: 191), liegt es jenseits des Erfahrungsraums sowohl der Produzenten als auch der Interpreten und gilt

terviews in die Untersuchung einbezogen, schließen sich als letzter Verfahrensschritt kontrastive Fallvergleiche an, bei denen die Ergebnisse einzelner Interviewanalysen einander gegenübergestellt werden. Die Auswahl der zu vergleichenden Fälle soll dabei den Prinzipien des maximalen bzw. des minimalen Kontrasts folgen. Beim maximalen Kontrast wird zum Zwecke des Vergleichs ein Fall ausgewählt, dessen Struktur möglichst große Unterschiede zum ersten Fallbeispiel aufweist; hier dient der Vergleich vor allem dazu, die jeweiligen fallspezifischen Besonderheiten deutlicher zu konturieren. Der Vergleich zweier Fälle mit minimalem Kontrast verfolgt demgegenüber das Ziel, anhand der Gemeinsamkeiten beider Fälle fallübergreifende Strukturen herauszuarbeiten." (Koller 1999: 175)

10 | Bohnsack bezieht sich in seiner Beschreibung auf die Erfahrung mit Gruppendiskussionen. 
in diesem Sinne als intersubjektiv nachvollziehbar. ${ }^{11}$ In dieser Hinsicht ist das Ziel der hier vorliegenden Analyse die Rekonstruktion kollektiven Wissens. Spätestens an dieser Stelle wird deutlich, dass sich die dokumentarische Methode und die Diskursanalyse hinsichtlich ihrer Prämissen bezüglich der Rekonstruktion impliziten Wissens gut miteinander verknüpfen lassen.

"Die diskursive Wissensanalyse richtet sich dabei nicht auf die produzierte Erkenntnis, auf das 'positive Wissen', sondern auf die produktiven Konstruktionsweisen und Wissenspraktiken, die die Bedingungen von Sagbarkeit und Sichtbarkeit bilden. Der analytische Gegenstand ist dann das implizite Wissen, das der Produktion expliziten Wissens zugrunde liegt." (Wrana 2012: 196)

Auch die Diskursanalyse geht davon aus, dass sich das implizite Wissen von den Intentionen der Akteure abhebt, was Tomke König für die Analyse von Interviews im Rahmen diskursanalytischer Verfahren expliziert:

"Für die Analyse der Interviews heißt das: Es wird nicht beansprucht, dass die Ergebnisse mit den Alltagsbedeutungen der Akteure überein stimmen. Vielmehr wird beschrieben, was in den Redeereignissen des Interviews geschieht. Welche Selbstverhältnisse und Machtverhältnisse werden in der Logik dieses Geschehens konstituiert? Statt in der 'Tiefe r nach Bedeutungen zu suchen, die den Alltagspraktiken innewohnen, werden Handlungs-, Denk- und Gefühlspraktiken auf der ,Oberfläche، dekonstruiert. [...] Entscheidend ist dabei also nicht, wie häufig ein bestimmtes Argument auftaucht, sondern nach welchen Regeln die Äußerungen geformt sind. Es wird gefragt, ob sich in diesen Regeln gesellschaftliche Diskurse (trans)formieren." (König 2008: 4788f.)

Die Interpretationen der Einstiegspassagen der Interviews (4.1.1) fokussieren eben auf gemeinsame »Regeln« des Sprechens über Jugendgewaltprävention. Zusammenfassend ist also festzuhalten, dass Diskursanalyse nicht als Methode zu verstehen ist, sondern als Forschungsperspektive oder auch -programm, in das Methoden integriert werden können (vgl. Angermüller 2014; Keller

11 Insofern als die befragten Fachpersonen sich an dem Wissen, das sie selbst konstruieren, orientieren, sind sie selbst als Interpretinnen bzw. Interpreten ihres eigenen Wissens anzusehen. An dieser Stelle scheidet sich die Methodologie der dokumentarischen Methode als interpretierende Methode von der Ethnomethodologie. Während die Ethnomethodologie ausschließlich die Interpretationsmethoden der Teilnehmenden eines sozialen Raums fokussiert, genauer: die "primären Sinnstrukturen" (Bergmann 1981: 12), an denen sich die Handelnden orientieren und die sie gleichzeitig mitproduzieren (vgl. auch Bergmann 1985; Patzelt 1987), fokussiert die Methodologie der dokumentarischen Methode den Sinngehalt jenseits von dem der Teilnehmenden (Forscherinnen bzw. Forscher und Beforschte). 
2001, 2010; Luutz 1994; Schwab-Trapp 2001). Dies wird insbesondere für die Erhebungsmethode des Interviews und damit einhergehende Interpretationsverfahren diskutiert, insofern als Interviews als »methodisch provozierte Redeereignisse « (König 2006: 4787) und demnach als diskursive Praktiken angesehen werden, während derer sich die Teilnehmenden unweigerlich diskursiv etablierter Wissensbestände bedienen, was schließlich in den Interpretationen berücksichtigt wird (vgl. auch Bender/Eck 2014). Zudem stellt sich die Frage, wie aus dieser Perspektive vom »Sprechen über« auf konkrete Handlungspraktiken geschlossen werden kann, was, wie bereits aufgezeigt worden ist, nur im Sinne einer analytischen Unterscheidung zwischen Diskurs und »diskursiven Praktiken« möglich ist. Eine der grundlegenden Prämissen, die die folgende Analyse leitet, resultiert aus einer sozialkonstruktivistischen Perspektive. Die Diskursforschung sieht Diskurse als »konstitutiv für das Soziale« an, insoweit sie »die soziale Welt nicht einfach abbilden; sie können soziale Realitäten >schaffen<, indem sie diese repräsentieren « (Angermüller 2014: 18f.). Es wird also davon ausgegangen, dass die Orientierungen der Fachpersonen an diskursiv erzeugten Wissensbeständen konstitutiv für die Wahrnehmung ihrer eigenen Praktiken sind (vgl. Bublitz et al. 1999: 13; Holstein/Miller 1993: 132; Keller 1997: 315, 2004: 68; Schmidt 2015: 110). Die Integration von Interviewverfahren in diskursanalytische Arbeiten ist im Gegensatz zur Integration ethnografischer Verfahren wenig etabliert. Studien, die sich diese Integration zunutze machen, bedienen sich insbesondere biografischer Interviews (vgl. Koller 1999; Reh 2003) sowie narrativer Interviews (vgl. Angermüller 2014), woran die Interpretation offener leitfadengestützter Interviews durchaus anschließen kann, wenn die Interviews eine hohe narrative Dichte aufweisen und sich (berufs-)biografische Bezüge als relevant erweisen. Ziel der vorliegenden Interpretationen ist die Rekonstruktion kollektiven Wissens mithilfe der dokumentarischen Methode im Sinne des konjunktiven Wissens als gemeinsamer Erfahrungshintergrund bzw. als gemeinsame Handlungsorientierungen. Die wissenssoziologische Diskursanalyse bezieht sich hierbei insoweit auf den Begriff des Deutungsmusters, als sie Diskurse als durch Deutungsmuster strukturiert ansieht, die wirkmächtige Sprecherpositionen, »Subjekt-Positionen«, konstituieren und dabei auch handlungsleitend wirken (vgl. Keller 2001). Dabei macht sie die Beobachtung, dass öffentliche Diskurse durch einen roten Faden - eine »gemeinsame Grunderzählung « - charakterisiert sind. Die hier vorliegende Arbeit interessiert sich für die Frage, inwiefern sich die Fachpersonen im Kontext von Jugendgewaltprävention im Sprechen über ihre Handlungskontexte eben auf eine solche "Story Line « beziehen und das Wissen, welches sie daraus beziehen, gebrauchen. 


\subsection{Feldzugang und Datenmaterial}

Die vorliegende Arbeit verknüpft zwei unterschiedliche Analyseebenen. Zum einen stehen die Interpretationen offener leitfadengestützter Interviews mit Fachpersonen im Kontext von Jugendgewalt im Vordergrund (4.1). Zum anderen werden Aussagen im Kontext des »Nationalen Präventionsprogramms Jugend und Gewalt« in Bezug auf die Forschungsfrage und vor dem Hintergrund der Ergebnisse interpretiert. Gegenstand sind dabei ein Transkript und zwei Protokolle von Konferenzen sowie das Dokument »Gesamtschweizerisches Präventionsprogramm Jugend und Gewalt« selbst (4.2). Die Ergebnisse der beiden Ebenen werden anschließend zusammengefasst und aufeinander bezogen (4.3). Schließlich wird die Analyse einer methodischen Reflexion unterzogen (4.4). Die Rekrutierung der Interviewpartnerinnen und Interviewpartner erfolgte nach dem Besuch der Forscherin der »1. Nationalen Konferenz >Jugend und Gewalt< am 09.03.2012 im Rathaus Bern - Wirksame Gewaltprävention in der Schweiz: Voneinander lernen über Sprachgrenzen und Staatsebenen hinweg « auf Basis von Listen der Austellerinnen und Aussteller sowie Teilnehmerinnen und Teilnehmer. Da Jugendgewaltprävention Gegenstand verschiedenster Fachbereiche ist und sich die Forschungsfrage auf die Orientierungsweisen der Fachpersonen an kriminalpolitischer Programmatik bezieht, bildet die vorliegende Arbeit ein breites Spektrum an Adressatinnen und Adressaten des »Nationalen Präventionsprogramms Jugend und Gewalt«ab. Daher wird in der Auswertung der Interviews fall- und themespezifisch kontrastiert. Fokus der Analyse ist, wie bereits festgehalten, die Rekonstruktion gemeinsamer Orientierungsmuster, die, wovon die dokumentarische Methode ausgeht, auf einem gemeinsam geteilten kollektiven Wissen basieren. In diesem Sinne stellt das »Nationale Präventionsprogramm Jugend und Gewalt« die Bedeutung eines gemeinsamen Vorgehens gegen Jugendgewalt heraus. Dieser kollektiven Adressierung von Verantwortung und somit der Heterogenität »des Diskurses « wird durch den Einbezug unterschiedlicher Fachbereiche in das Sample Rechnung getragen. Das Sample bildet damit eine sog. »Präventionslandschaft« (CremerSchäfer 2016: 19; Widersprüche 2016: 3) im schweizerischen Kontext von Jugendgewaltprävention ab. Die Erhebung erfolgte im Frühjahr 2012 durch die Forscherin. Der dafür eingesetzte Interviewleitfaden wurde im Rahmen eines Workshops im Herbst 2011 erstellt. Auch die Transkriptionen sowie die Erstellung thematischer Abläufe der Interviews erfolgten durch die Forscherin im Sommer 2012. Die Interpretationen der Interviews wurden zu einem guten Teil in Interpretationsgruppen und verschiedenen Foren vorgetragen und zur Diskussion gestellt. Im Folgenden wird auf die Überlegungen eingegangen, die den Einsatz eines Interviewleitfadens betreffen. Anschließend werden der Interviewleitfaden selbst und dessen Erstellung besprochen; schließlich wird eine Übersicht über die interviewten Fachpersonen gegeben. Ziel der Inter- 
pretationen ist, wie bereits erwähnt, die Rekonstruktion kollektiven Wissens bezüglich »Jugendgewaltprävention« aus der Perspektive von Fachpersonen, die in diesem Kontext tätig sind, wobei diesbezügliche Problematisierungsweisen von Jugendgewalt sowie daran anschließende Verständnisse bezüglich Jugendgewaltprävention bzw. Präventionskonzepte relevant sind. Die Interviews wurden im Kontext eines »Präventionsdiskurses « interpretiert und dabei v.a. in den Kontext eines konkreten Programms, des »Nationalen Programms Jugend und Gewalt«, gestellt. Aufgrund dieser thematischen Eingrenzung und dem damit verbundenen Bestreben, im Sinne der dokumentarischen Methode vergleichend zu interpretieren, ist der Einsatz offener leitfadengestützter Interviews sinnvoll: Diese bieten den Befragten einerseits Raum für subjektive Relevanzen und Erzählungen und geben den Erzählungen andererseits eine (mögliche) thematische Struktur. Im Sinne der dokumentarischen Methode zielte die Erhebung auf die Generierung möglichst offener Erzählungen der Befragten über deren Tätigkeitsbereich und die dabei relevanten Aspekte bezüglich Jugendgewaltprävention. Der Interviewleitfaden diente der Forscherin also primär als Orientierung an den als relevant gesetzten Themenbereichen und nicht als Eingrenzung derselben. In diesem Sinne

"verträgt die Erhebung von subjektiven Konzepten, subjektiven Theorien, Deutungsmustern, Orientierungen, Positionierungen eine gewisse Strukturierung z.B. in Form eines Leitfadens für die Interviewführung. Interpretationen lassen sich auch an kleineren Textsegmenten festmachen, die durchaus auf Vorgaben von Interviewenden hin produziert werden können, sofern die Offenheit gewahrt wird und der Leitfaden angemessen gestaltet ist und angemessen gehandhabt wird." (Helfferich 2011: 38)

Der Interviewleitfaden wurde im Rahmen eines Workshops gemeinsam in der Gruppe generiert und anschließend von der Forscherin überarbeitet. ${ }^{12}$ Er enthält 15 Leitfragen, die sich auf verschiedene Themenschwerpunkte beziehen, wie folgend erläutert wird.

I "Soziales Problem «: Jugendgewalt - Was ist Ihrer Erfahrung nach das Problem?

- Inhaltliche Aspekte: Betroffenheit; Entwicklung; Schweiz; Migration; Stellenwert

- Nachfragen: Wer ist betroffen? Wer sind die Leidtragenden? Welchen Stellenwert hat das Problem in der Gesellschaft? Wie hat sich das Problem in den letzten Jahren entwickelt?

12 | "Workshop Qualitative Leitfadeninterviews. Strukturierung vs. Offenheit. Von der Forschungsfragestellung zum Gesprächsleitfaden“ bei Dr. Jan Kruse am 24.-26. November $2011 \mathrm{im}$ Weiterbildungszentrum der Freien Universität Berlin (vgl. als Grundlage Kruse 2011). 
- Aufrechterhaltungsfragen: Gibt es sonst noch etwas? Und sonst? Und weiter? Und dann? Können Sie das genauer beschreiben? Was meinen Sie damit konkret?

Die Einstiegsfrage zielt auf die Erfahrungsebene der Fachpersonen hinsichtlich ihres beruflichen Kontexts. Die Frage konstruiert Jugendgewalt als Problemzusammenhang, der aus der beruflichen Perspektive der Fachpersonen erläutert werden soll. Mit dieser Frage gehen also bestimmte Annahmen der Forscherin über diesen Problemzusammenhang einher, wie beispielsweise die Annahme, dass die Fachpersonen über diejenigen Personen oder Personengruppen sprechen, die von »dem Problem« betroffen sind und als Adressatinnen und Adressaten der jeweiligen Institution oder Organisation in den Blick geraten. Eine andere Annahme ist hingegen, dass die Fachpersonen »das Problem« auf gesellschaftskritischer Ebene reflektieren. Die Forscherin bedient sich bei der Konstruktion des Interviewleitfadens sowie bei der Interviewführung selbst eines Wissens, das sie für den Kontext von Jugendgewaltprävention als relevant erachtet. Ziel der Erhebung ist es jedoch nicht gewesen, den Leitfaden möglichst genau umzusetzen; der Leitfaden dient der Forscherin vielmehr als Orientierungshilfe. Er strukturiert den Interviewverlauf, insbesondere wenn es an narrativen Passagen mangelt. Schließlich gewährleistet der Einsatz eines Leifadens potenziell eine Vergleichbarkeit der Interviews, was im Sinne der dokumentarischen Methode ist. ${ }^{13}$

II "Diskurs«: Wenn Sie auf die letzten zehn Jahre zurückblicken: Inwiefern hat sich die Thematisierung von Jugendgewalt verändert?

- Inhaltliche Aspekte: Öffentlicher Diskurs; Expertendiskurs; Medien; Erwachsenenthema; Extremfälle; Hype; Fußball

- Nachfragen: Warum ist das Thema für die Schweiz so zentral? Wer thematisiert Jugendgewalt in besonderer Weise? Für wen ist das Thema so zentral?

Die zweite Leitfrage transportiert die Annahme, dass sich die (öffentlich-mediale und politische) Thematisierung von Jugendgewalt in den letzten zehn Jahren verändert hat. Hier könnte von Interesse sein, auf welche »Diskurse« sich die Fachpersonen beziehen bzw. welche »Diskurse« ihre Wahrnehmung und Problematisierungsweisen strukturieren. Diese Leitfrage soll zudem bewirken, dass ein Schweiz-spezifischer Horizont eingefangen wird.

III "Gewaltformen«: Welche Formen von Gewalt gibt es Ihrer Erfahrung nach?

- Inhaltliche Aspekte: Qualitative Formen von Gewalt; Quantität; Messbarkeit; Orte

- Nachfragen: Wie kann man das messen? Was sind weitere Aspekte? Wo lässt sich Gewalt beobachten?

13 | Die Aufrechterhaltungsfragen werden im Folgenden nicht mehr aufgeführt. 
Die dritte Leitfrage zielt auf eine Art von Definition des Gewaltbegriffs ab. Sie soll dazu anregen, allgemeine Erfahrungen bezüglich Gewalt als Phänomen zu thematisieren und eine Differenzierung des Gewaltbegriffs bzw. des dahinterliegenden Verständnisses vorzunehmen. Eine Differenz zwischen »Gewalt « und »Jugendgewalt« ist wichtig, um den spezifischen Kontext der »Jugendgewaltprävention in der Schweiz« von anderen Problemzusammenhängen, wie etwa allgemeiner Kriminalprävention, abzugrenzen.

IV "Formen von Jugendgewalt»: Jugend und Gewalt - wie hängt das zusammen?

- Inhaltliche Aspekte: Qualitative Formen von Gewalt; Quantität; Messbarkeit; Orte

- Nachfragen: Wie kann man das messen? Was sind weitere Aspekte? Wo lässt das beobachten? Welche Aspekte sind besonders relevant?

Auch die Frage nach dem Zusammenhang von Jugend und Gewalt zielt auf eine Spezifik von Jugendgewalt in Abgrenzung zu Gewalt ab und soll Definitionen oder gesellschaftskritische Reflexionen bewirken.

V "Ursachen«: Worin sehen Sie die Ursachen von Jugendgewalt?

- Inhaltliche Aspekte: Faktoren; Entstehung; Lernen; persönliche Entwicklung; Gesellschaft; Migration

- Nachfragen: Kann man Gewalt lernen? Gibt es persönliche oder gesellschaftliche Faktoren? Kann man Gewalt erklären?

Die Frage nach den Ursachen von Jugendgewalt zielt auf die Reflexion von Ursachen von Jugendgewalt ab, denen im Allgemeinen ein hoher Stellenwert beigemessen wird. Die zentrale Frage ist, welche Relevanz die Ursachen aus der Sichtweise der jeweiligen Fachpersonen für ihren jeweiligen Arbeitskontext haben.

VI „Funktion«: Wie stellen Sie sich eine Gesellschaft ohne Gewalt vor?

- Inhaltliche Aspekte: Normalität; Vorbilder

- Nachfragen: Hat Gewalt Ihrer Meinung nach eine gesellschaftliche Funktion? Was wünschen Sie sich?

Diese Frage zielt auf eine Beschreibung ab, wie Gesellschaft aus der Perspektive der Fachpersonen idealerweise sein könnte, und soll damit Aufschluss geben über deren normative Erwartungen an Gesellschaft oder Gemeinschaft. Des Weiteren könnte die Frage, die eigentlich eine Utopie thematisiert, rhetorisch aufgefasst werden und in dieser Lesart dazu einladen, die bisher entwickelten Perspektiven auf Gewalt bzw. Jugendgewalt zu reflektieren und zu prüfen sowie Begriffe und Problematisierungsweisen zu schärfen. 
VIII "Legitimität«: Gibt es Ihrer Erfahrung nach legitime Gewaltformen?

- Inhaltliche Aspekte: Normalität; Orte/Bereiche; soll geben vs. gibt es? Utopie vs. Empirie

- Nachfragen: Wann ist Gewalt legitim? Wie viel Gewalt ist normal?

Auch die Frage nach legitimen Gewaltformen lädt die befragten Fachpersonen dazu ein, kontrastierende Deutungen und Problematisierungsweisen von Gewalt bzw. Jugendgewalt vorzunehmen und dabei ihre eigenen Erfahrungen bezüglich ihres jeweiligen Tätigkeitsbereichs und dessen Relevanzen zu schildern. Auch diese Frage ermöglicht eine weitere Vertiefung der Begriffe.

VIII „Profession«: Inwiefern haben Sie im Rahmen Ihrer Tätigkeit mit Jugendgewalt zu tun?

- Inhaltliche Aspekte: Arbeitsalltag; Formen der Gewalt; Erfahrung; Macht; Einfluss

- Nachfragen: Können Sie mir Ihren Arbeitsalltag beschreiben? Wie gehen Sie damit um, wenn Sie eine Jugendliche oder ein Jugendlicher anpöbelt?

Diese Frage zielt darauf ab - falls bisher noch nicht erfolgt -, konkret zu erfassen, inwiefern Jugendgewalt in den jeweiligen Fachbereichen relevant ist, sie soll zu einer Beschreibung der Tätigkeitsbereiche einladen.

IX "Fremdeinschätzung»: Welche Probleme haben Ihrer Erfahrung nach Jugendliche heute?

- Inhaltliche Aspekte: Entwicklungsaufgaben; Herausforderung; Schweiz

- Nachfragen: Welche Erwartungen haben Jugendliche? Welche Alternativen haben Jugendliche?

$X$ "Fremdeinschätzung/Motivation«: Jetzt würde mich Ihre Erfahrung hinsichtlich der Motivation Jugendlicher interessieren. Warum greifen Jugendliche zu solchen Mitteln?

- Inhaltliche Aspekte: Aggression; Kompensation; Reaktion; Alternativen

- Nachfragen: Auf wen oder was reagieren Jugendliche? Gegen wen oder was richtet sich Gewalt?

Diese beiden Leitfragen zielen darauf ab, zu erfassen, inwieweit die befragten Fachpersonen Perspektiven Jugendlicher einnehmen - sich in sie hineinversetzen. Darüber hinaus ist es in diesem Zusammenhang von Interesse, welche (normativen) Erwartungen die befragten Fachpersonen an Jugendliche haben, wobei auch das Verständnis von »Jugend « bzw. »Jugendphase« relevant ist. Der Gegenstand bzw. der Begriff der Gewalt bzw. der Jugendgewalt kann durch diese Frage möglicherweise weiter differenziert und reflektiert werden. 
XI „Visibilität»: Wie erkennen Sie das Problem?

- Inhaltliche Aspekte: Intervention; Stigmatisierung

- Nachfragen: Können Sie mir ein Beispiel für eine problematische Situation geben? Wann ist ein Eingreifen erforderlich?

Die Frage nach der Erkennbarkeit »des Problems« richtet den Fokus auf das jeweilige Tätigkeitsfeld und soll Erzählungen oder Beispiele generieren. Erzählungen über die »Visibilität« von »Problemen« geben Aufschluss über das Verhältnis der Fachpersonen zu den Adressatinnen und Adressaten ihres Fachbereichs sowie über die Relevanzen der jeweiligen Problematisierungsweisen. Hier sollen die Handlungspraktiken der Befragten themtisiert werden.

XII "Verantwortung": Wer sind die Verantwortlichen?

- Inhaltliche Aspekte: Jugendliche; Institutionen; Schule; Eltern; Familie

- Nachfragen: Wo wurde versagt? Inwieweit kann man Jugendliche in die Verantwortung nehmen?

Die Frage nach der Verantwortung ermöglicht eine Überleitung zum Thema »Prävention«. Sie nimmt an, dass es Personen bzw. Personengruppen gibt, die verantwortlich sind, und zielt darauf ab herauszufinden, welche Personen bzw. Personengruppen als Adressatinnen und Adressaten von Jugendgewaltprävention angesehen werden.

XIII "Prävention«: Wie lässt sich Ihrer Meinung nach das Problem lösen?

- Inhaltliche Aspekte: Ziele von Prävention; Inhalt von Prävention; Bedingungen dafür, dass Prävention gelingt; Exklusion; Inklusion

- Nachfragen: Wie wollen Sie die Jugendlichen erreichen? Welche Jugendlichen wollen Sie erreichen? Was sind die Ziele von Prävention? Wie kann Prävention gelingen?

Die Frage nach der »Problemlösung « zielt auf konkrete Praktiken und Konzepte der Prävention bzw. Intervention oder sogar auf »gute Präventionsarbeit«. Auch kann hier wiederum erschlossen werden, welche Personen bzw. Personengruppen überhaupt Adressatinnen und Adressaten der jeweiligen Fachpersonen sind. Ziel ist letztlich, Verständnisse von Prävention zu (re-)konstruieren.

XIV "Strafe«: Inwieweit können Jugendliche Ihrer Meinung nach für ihr Handeln zur Verantwortung gezogen werden?

- Inhaltliche Aspekte: Strafsystem; Jugendschutz; Verhältnis von Prävention und Repression 
- Nachfragen: Was sollte strafrechtlich gesehen verändert werden? In welchem Verhältnis stehen Prävention und Repression? Brauchen wir härtere Gesetze?

Die Fragen nach der Verantwortung Jugendlicher sowie nach der Bedeutung von Gesetzen zielen darauf ab, den Stellenwert von Prävention aus der Perspektive der Fachpersonen zu erfassen. Die Bestrafung konkreter Individuen bzw. Repression wird in der Literatur oft als eine der Prävention gegenläufige Maßnahme konzeptualisiert. Diese Frage zielt also letztlich darauf ab, Präventionsverständnisse weiter zu differenzieren und in einen weiteren gesellschaftlichen Rahmen zu stellen. Die Frage danach, ob wir härtere Gesetze brauchen, könnte auch provokativ wirken. ${ }^{14}$

XV „Alternativen «: Inwiefern können Ihrer Meinung nach Erwachsene Vorbilder sein?

- Inhaltliche Aspekte: Frieden, Identität, Idole, Rolle der Erwachsenen (Fachpersonen)

- Nachfragen: Welche Alternativen gibt es? Sehen Sie sich als Vorbild?

Mit der Frage nach der Vorbildfunktion von Erwachsenen sollen die (normativen) Erwartungen der Interviewten erfasst werden, die nicht nur Fachpersonen, sondern selbst auch Erwachsene sind. Die Möglichkeit eines Perspektivenwechsels sowie die Positionierung zu womöglich provozierenden Fragen sollen den Fachpersonen weitere Differenzierungsmöglichkeiten bieten.

Die Leitfragen und Nachfragen enthalten Wissen und bestimmte Annahmen über den Kontext von Jugendgewaltprävention und sind somit nicht als »neutral« oder gar objektiv zu bewerten, was in der hier vorliegenden Arbeit auch nicht als möglich erachtet wird. Die Forscherin bzw. Interviewerin ist in ihren Praktiken nicht unabhängig von Diskursen, im Gegenteil: Sie muss Wissen über einen bestimmten Kontext gebrauchen, um dem Ziel des Leitfadens, mithilfe von Fragen Erzählungen, Kontrastierungen und Perspektivenverschiebungen anzustoßen und zentrale Relevanzen bezüglich Jugendgewaltprävention aus der Perspektive der Fachpersonen zu generieren, gerecht zu werden. Eine Konfrontation mit den Relevanzen, Annahmen und Problemzusammenhängen der Forscherin ermöglicht es den Befragten, sich abzugrenzen und (in dieser Abgrenzung) eigene Relevanzen und Problemzusammenhänge zu explizieren. Der Leitfaden soll den Interviewverlauf dabei nur grob strukturieren. Beschreibungen und Erzählungen sollen vielmehr durch situative Nachfragen

14 | "Die Zulassung von Suggestivfragen ist damit verbunden, dass als Teil einer aktiven Intervention die Reaktion von Befragten auf Unterstellungen bewusst und explizit provoziert, registriert und interpretiert werden soll; die Suggestivfrage funktioniert dann so wie ein kleines soziales Experiment." (Helfferich 2011: 106) 
angeregt werden: »Fragen haben die Funktion, zu Erzählungen bzw. Äußerungen zu motivieren und so die Kommunikation aufrecht zu erhalten und Interesse und Verstehen zu zeigen.« (Helfferich 2011: 102) Auf Fragen, die auf eine konkrete Positionierung gegenüber dem Programm abzielen, wurde bewusst verzichtet, um die Bedeutungen und Relevanzen der jeweiligen Fachpersonen $\mathrm{zu}$ fokussieren. Die Interviewpartnerinnen und Interviewpartner sind durch folgendes Anschreiben rekrutiert worden. Nicht nur an den Fragen des Leitfadens wird also die Unmöglichkeit ersichtlich, sich als Forscherin dem Wissen zu entziehen, das sie eigentlich zum Gegenstand ihrer Untersuchung macht, sondern bereits durch das Anschreiben, das die Erwartungen der Befragten an das Interview vorstrukturiert:

„Promotionsvorhaben zum Thema Jugendgewaltprävention، in der Schweiz.

Sehr geehrte/r Frau/Herr Name،, ich bin Soziologin (Dipl.-Soz.) und promoviere am Soziologischen Institut der Universität Zürich. Im Rahmen meiner Doktorarbeit beschäftige ich mich mit dem Thema Jugendgewaltprävention، aus soziologischer Perspektive.

Dabei ist es mir ein zentrales Anliegen, das Wissen und die Erfahrungen derjenigen Menschen einzufangen, die in diesem Bereich praktisch tätig sind, und ich bin auf der Suche nach InterviewpartnerInnen.

In den qualitativen Leitfadeninterviews interessiere ich mich insbesondere für Ihre persönliche Praxiserfahrung, also Ihren tätigkeitsbezogenen Wissens- und Erfahrungsraum sowie Ihre diesbezüglichen Meinungen zum Thema Jugendgewalt.

In den Interviews werde ich verschiedene offene Fragen stellen, bei denen ich Sie grundsätzlich bitte, mir all das zu erzählen, was für Sie relevant und wichtig ist. Aufgrund der offenen Gesprächssituation nehmen die Interviews ca. eine Stunde in Anspruch. Die Interviews werden für die spätere Auswertung aufgenommen. Selbstverständlich verwende ich das Interviewmaterial in meiner Studie streng vertraulich und anonym. Bei Interesse schicke ich nach Vollendung meiner Doktorarbeit selbstverständlich Ihnen auch die betreffenden Passagen zu.

Ich würde mich sehr darüber freuen, wenn Sie Interesse und Zeit für ein Interview haben, sodass ich mein Promotionsvorhaben erfolgreich abschliessen kann, und möchte Sie dazu herzlich einladen, mit mir Kontakt aufzunehmen, um eventuelle Fragen oder dergleichen in einem persönlichen Gespräch abzuklären.

Freundliche Grüsse"

Die Forscherin stellt sich im Rahmen dieses Anschreibens als Wissenschaftlerin dar, die sich qualifizieren will und Mitarbeiterin an einer Universität ist. Damit schreibt sie die Fachpersonen nicht nur als »Expertinnen« bzw. »Experten « für Jugendgewaltprävention an, sondern stellt auch sich selbst als »Expertin« dar, was den Interaktionsverlauf des Interviews maßgeblich beeinflusst, insoweit diesbezügliche Erwartungen relevant werden (vgl. Bogner/ 
Menz 2009; Helfferich 2011: 119ff.). Das zeigt sich auch in den hier vorliegenden Interviews. Die Darstellung als Wissenschaftlerin eines bestimmten Fachbereichs (Soziologie) generiert Erwartungen hinsichtlich eines bestimmten Wissens (»soziologisches« Wissen), was sich ebenfalls als relevant erweist, insofern die Fachpersonen bestimmte Annahmen an dieses Wissen formulieren und ihren eigenen Wissenshintergrund davon abgrenzen. Darüber hinaus adressiert es Personen, die im Bereich der Jugendgewaltprävention »praktisch tätig " sind bzw. stellt dies als ein Auswahlkriterium dar. Neben der Praxiserfahrung erachtet die Forscherin laut diesem Anschreiben auch die »Meinungen zum Thema Jugendgewalt« als bedeutsam. Die hier vorliegende Arbeit wird zeigen, dass sich die befragten Fachpersonen auf diesen verschiedenen Ebenen - der persönlichen (privaten), der fachlichen und berufsbiografischen Erfahrung sowie der persönlichen Meinung - positionieren, was ihre eigenen Deutungs- und Problematisierungsweisen mitstrukturiert. Daraus kann geschlossen werden, dass das Anschreiben bzw. die Art und Weise der Rekrutierung von Interviewpartnerinnen und Interviewpartnern deren Erwartungen und Deutungen maßgeblich vorstrukturiert. Diese Beobachtung muss, insbesondere aus diskursanalytischer Perspektive, von der Forscherin reflektiert werden, da sie ihre eigenen Interpretationen betrifft. Das Sample der Studie bildet die Heterogenität der im Kontext von Jugendgewaltprävention angesiedelten Fachbereiche ab, die auch vom »Nationalen Präventionsprogramm Jugend und Gewalt« in ihrer Expertise adressiert werden. Bei der konkreten Auswahl der Interviewpartnerinnen und Interviewpartner ist darauf geachtet worden, dass es sich möglichst um Fachpersonen handelt, die regelmäßig mit Jugendlichen arbeiten. Da die Kontaktaufnahme teilweise über die Organisationen bzw. Institutionen erfolgte, ist die Forscherin jedoch auch an Personen weitergeleitet worden, die nicht (mehr) regelmäßig mit Jugendlichen arbeiten, weil sie (inzwischen) primär leitend tätig sind. Dies ist bei Herrn Tanner der Fall, der nach einer »klassischen« Karriere als Jugendarbeiter in der offenen und aufsuchenden Jugendarbeit inzwischen leitend tätig ist und nur noch im Rahmen von Veranstaltungen direkt mit Jugendlichen zu tun hat. Frau Blaum ist in der Einrichtung, die Herr Tanner leitet, als Jugendarbeiterin tätig. Eine weitere Beobachtung, die das Selbstverständnis der Forscherin irritiert hat, ist, dass sich Jugendgewaltprävention empirisch nicht selbstverständlich als Arbeit mit Jugendlichen zeigt. Ein Schwerpunkt einiger Institutionen bzw. Organisationen im Kontext von Jugendgewaltprävention ist die Arbeit mit Familien oder anderen Institutionen bzw. »Systemen « wie Schulen. Dies betrifft insbesondere Frau Schock, die primär als Familientherapeutin und sekundär in der aufsuchenden Jugendarbeit tätig ist. Herr Unterwasser, der u.a. Workshops an Schulen gibt, betont im Kontext von Jugendgewaltprävention die Bedeutung der Arbeit mit Erwachsenen bzw. mit anderen Institutionen, die sich auf bestimmte Aspekte spezialisiert haben. Er misst der wissenschaftlichen Erfor- 
schung von Jugendgewalt, insbesondere aus psychoanalytischer Perspektive, einen sehr viel höheren Stellenwert bei als der Arbeit mit Jugendlichen. Herr Gold wiederum arbeitet trotz seiner Leitungsfunktion in einer Organisation, die sich auf Jugendgewaltprävention spezialisiert hat, primär mit Jugendlichen. Er leitet Workshops an Schulen und unterrichtet Kampfsport. Auch Herr Auto gibt als Leiter einer Organisation primär Workshops an Schulen. Herr Ifon arbeitet als Leiter der Jugendpolizei eines Kantons beinahe ausschließlich vom Schreibtisch aus, hat aber eine Karriere als »Gassenpolizist« hinter sich. Herr Schäfer schließlich arbeitet als Jugendrichter zu einem Gutteil mit straffällig gewordenen Jugendlichen. Das Sample deckt somit »primäre«, »sekundäre « und »tertiäre « Prävention ab - eine Unterscheidung, die Gerald Caplan in den 196oern im Kontext der Gesundheitsprävention eingeführt hat (vgl. Caplan 1964) und die heute nicht nur in sozialpädagogischen, sondern auch in kriminalpolitischen Kontexten als relevant erachtet wird (vgl. Brüchert 2010). Diese Differenzierung ist für die Analyse der hier vorliegenden Arbeit nur insofern relevant, als sie das beforschte Feld als relevant setzt.

"Kriminologische Differenzierungen zwischen positiver und negativer General- und Spezialprävention taugen für eine sozialwissenschaftliche Analyse ebenso wenig wie die (sozial)pädagogische Unterscheidung von primärer, sekundärer und tertiärer Prävention. Ein sozialwissenschaftlicher Begriff von 'Prävention ‘ hätte eben diese wissenschaftlichen Definitionsversuche als wichtigen Teil des Feldes, das es zu beschreiben gilt, mit in den Blick zu nehmen. [...] Ein sozialwissenschaftlicher Begriff von Prävention hätte aber vor allem auch die Auswirkungen dieser Definitionsversuche auf die Problemwahrnehmung der verschiedenen Akteure in den Blick zu nehmen." (Ebd.: 272f.)

Gegenstand der Analyse sind demnach die Deutungsweisen aus der Perspektive der befragten Fachpersonen und auf kriminalpolitischer Ebene. Die Interviews sind selbstverständlich anonymisiert. Einverständniserklärungen liegen vor.

Kurzübersicht über die befragten Fachpersonen

- Herr Unterwasser: Fachbereichsleiter

- Frau Schock: Familientherapeutin und Jugendarbeiterin

- Herr Gold: Leiter einer privaten Organisation

- Herr Vonderstatt: Leiter einer städtischen Jugendarbeit

- Herr Tanner: Leiter einer offenen Jugendarbeit

- Frau Blaum: Jugendarbeiterin

- Herr Auto: Geschäftsführer einer Privaten Organisation

- Herr Ifon: Leiter der Jugendpolizei einer Stadt

- Herr Schäfer: Jugendrichter 


\section{Datenauswertung}

\section{1 (Jugendgewalt-)Prävention aus der Perspektive DER FACHPERSONEN}

Die Besonderheit folgender Interpretationen besteht zunächst in der methodischen Reflexion hinsichtlich der Adressierung der Befragten als Expertinnen bzw. Experten für Jugendgewaltprävention. Wie gehen die Befragten mit diesbezüglichen Zuschreibungen um und welche (eigenen) Relevanzen entwickeln sie vor diesem Hintergrund (4.1.1)? Laut Bogner und Menz (2009) spielen für die Interaktionsstruktur der Interviews und damit für die Rekonstruktion von Deutungswissen nicht nur die Zuschreibungen der Interviewerinnen bzw. Interviewer hinsichtlich der Expertise der Befragten eine zentrale Rolle, sondern auch die Zuschreibungen der Befragten hinsichtlich der Expertise, der Ziele und der Motivationen der Forscherin, die in diesem Fall auch die Interviews führt. Die Autoren unterscheiden idealtypisch zwischen sechs verschiedenen Zuschreibungen seitens der Expertinnen und Experten: Die Interviewerin bzw. der Interviewer als Co-Expertin bzw. Co-Experte, als Expertin bzw. Experte einer anderen Wissenskultur, als Laie, als Autorität, als Komplizin bzw. Komplize sowie als potenzielle Kritikerin bzw. Kritiker. Sie nehmen an, dass die Rekonstruktion von Deutungswissen bestimmten Bedingungen - Dynamiken des Interaktionsverlaufs - unterliegt. Die Zuschreibung als Expertin bzw. Experte einer anderen Wissenskultur zwingt die Befragten dazu, ihren Orientierungsrahmen zu explizieren, was jedoch auch die Gefahr birgt, zu sehr dem Relevanzsystem der Interviewerin verhaftet zu bleiben. Die Zuschreibung als Laie zwingt die Befragten ebenfalls zur Explikation ihres Handlungs- und Deutungskontextes und legt ihnen einen allgemeinen Erzählzwang auf, auf den die Interviewerin bzw. der Interviewer mit »naiven« Fragen reagieren kann. Problematisch kann hier die Wahrnehmung der Interviewerin bzw. des Interviewers als »unwillkommen « sein, was dazu führen kann, dass die Expertinnen und Experten Fragen weniger ernst nehmen und rasch abhandeln. Zudem könnte der Erzählzwang zu Monologen führen, die durch die Interviewerin bzw. den Interviewer nur noch schwer steuerbar sind. Schließlich geht mit der Zuschreibung als Komplizin bzw. Komplize möglicherweise ein hoher Vertrauensge- 
winn einher. Unter Bezugnahme auf gegenseitige Zuschreibungsweisen in den Einstiegspassagen wird in der hier vorliegenden Arbeit zunächst die Verknüpfung von Interviewforschung und Diskursanalyse einer kritischen Reflexion unterzogen, woraus sich aber auch eine Sichtung der relevanten Themen und fallspezifischen Vergleichshorizonte für die weiteren Interpretationen ergibt. Eine Differenzierung zwischen den eigenen und zugeschriebenen Relevanzen der Jugendgewaltprävention erfolgt über die Reflexion der Interviews als Interaktionen. Diese Reflexionsmöglichkeit wird dadurch unterstützt, dass bei den Interpretationen der Einstiegspassagen nach zwei unterschiedlichen »Einstiegsfragen« unterschieden werden kann. Dabei bietet die Frage »Jugendgewalt - Was ist Ihrer Erfahrung nach das Problem?« eine sehr viel stärkere Kontrastfolie als die Aufforderung, offen über den jeweiligen Tätigkeitsbereich zu erzählen. Die Forscherin hat sich während des Erhebungsprozesses für den Wechsel zur Erzählaufforderung entschieden, um die Narrationsdichte potenziell zu erhöhen und die Aufmerksamkeit für die Relevanz der je spezifischen Handlungskontexte und, damit verbunden, der je eigenen Problematisierungs- und Lösungsweisen zu schärfen. Außerdem hat die Konfrontation mit der zunächst gewählten Einstiegsfrage eine kritische Auseinandersetzung mit dieser Frage auf teilweise emotionaler Ebene bewirkt, was zu einer »unguten« Gesprächssituation beigetragen hat. Die Reflexion dieses Eingriffs in den Erhebungsprozess wird nun einleitend genutzt, um einen Bogen zwischen den methodischen Herausforderungen und den Interpretationen der hier vorliegenden Arbeit zu spannen. Bezüglich der grundlegenden und zentralen Orientierungsweisen der befragten Fachpersonen lassen sich hinsichtlich dieser Intervention keine Unterschiede erkennen. Dies wird auch daran deutlich, dass die zunächst gewählte Einstiegsfrage während der Erhebungsphase nicht gänzlich aufgegeben, sondern verschoben worden ist. Den Befragten wird also nichtsdestotrotz die Möglichkeit gegeben, sich zu dieser Frage zu positionieren. Eine der zentralen Beobachtungen dieser ersten Interpretationen ist, dass die Befragten die Adressierung als Expertin bzw. Experte für Jugendgewaltprävention relativieren. Dabei zeigt sich in der Tendenz, dass sich die Befragten zunächst von der Zuschreibung distanzieren und ihre Expertise anschließend reformulieren. In diesem Relativierungsprozess deutet sich eine grundlegende Ambivalenz an: Die befragten Fachpersonen verstehen Jugendgewaltprävention nicht als zentralen Bestandteil ihrer Expertise und damit verbundenen Problematisierungs- und Deutungsweisen, sehen sich jedoch dazu angehalten, genau dies nach außen hin zu rechtfertigen. Bemerkenswert ist außerdem, dass die befragten Fachpersonen im Sprechen über Jugendgewaltprävention zwischen verschiedensten Sprecherpositionen wechseln: Ihre Problematisierungs- und Deutungsweisen sind geprägt von Erfahrungen, die sich nicht nur auf ihre konkreten Arbeitskontexte beziehen, sondern aus biografischen Erlebnissen, gesellschaftskritischen Haltungen, privaten Meinungen etc. resultieren. 


\subsubsection{Interpretation der Einstiegspassagen: Doing Expertise}

Die Frage nach den Orientierungsweisen von Fachpersonen an kriminalpolitischer Programmatik führt auf analytischer Ebene zu der Frage nach den Problematisierungsweisen von Jugendgewalt und daran anschließenden Präventionsverständnissen im Sinne von (Un-)Möglichkeiten »präventiver« Handlungspraktiken. Ziel der vorliegenden Interpretationen ist demzufolge die Rekonstruktion kollektiven Wissens über Jugendgewalt im Kontext von Jugendgewaltprävention und vor diesem Hintergrund die Frage nach Möglichkeiten und Grenzen von Jugendgewaltprävention in den je spezifischen Handlungskontexten. Im Vordergrund stehen hier Konstruktionsweisen von Prävention unter Bezugnahme auf Deutungen von Jugendgewalt. Diese Vorgehensweise impliziert, Prävention beziehe sich auf Jugendgewalt als (problematischen) Gegenstand. Mit der Rekonstruktion kollektiven Wissens geht die Reflexion der jeweiligen Orientierungsrahmen einher, was über die Reflexion der Adressierungsweisen in der Interviewpraxis unterstützt wird. Dabei deuten sich bereits auch die jeweiligen Expertisen sowie diesbezüglich als relevant gesetzten Problematisierungsweisen an. Vergleichenden, primär themenspezifischen Interpretationen gehen also zunächst fallspezifische Interpretationen voraus. Der Spezifik des Falls wird in den darauf folgenden, primär themenspezifischen Interpretationen jedoch Rechnung getragen. In dieser abwechselnden Gewichtung der Fokusse wird kollektives Wissen im Sinne gemeinsamer, durch Diskurse strukturierter Erfahrungen und Handlungspraktiken rekonstruierbar. Wie bereits expliziert sind Interviews aus diskursanalytischer Perspektive selbst als diskursive Praktiken anzusehen - als »Diskursivierung von Jugendgewaltprävention « -, die von der Forscherin hervorgebracht werden: Indem sie die Fachpersonen im Kontext von Jugendgewaltprävention als Expertinnen und Experten adressiert bzw. sich bestehender Adressierungsweisen und Positionierungen bedient, reproduziert sie Diskurse. Über Jugendgewalt zu forschen, bedeutet also, selbst »im Kontext des gesellschaftlichen Diskurses über Jugendgewalt« (Steiner 2002: 15) zu stehen. Dieser reflexive Weg eröffnet Blicke auf Prävention als Handlungspraktik, insofern die Betrachtung von Interviews als Interaktionen durchaus Schlüsse auf Aspekte alltäglicher Praktiken erlaubt (vgl. Neumann-Braun/Deppermann 1998). Gegenstand folgender Interpretationen ist somit zunächst - fallspezifisch - der Diskursraum der Interviews: In welcher Beziehung stehen diese zu Diskursfragmenten, an welche die Forscherin im Rahmen der Durchführung der Interviews (Anschreiben, Leitfragen und Nachfragen) selbst anknüpft? Eine der grundlegenden Beobachtungen ist, dass die befragten Fachpersonen tendenziell zunächst eine gesellschaftskritische Position einnehmen, indem sie Jugendgewalt als gesellschaftspolitisch überformtes Konstrukt entwickeln. Dies lässt sich zum Gutteil auf die Einstiegsfrage zurückführen, wobei festgehalten werden soll, 
dass diese gesellschaftskritischen Positionierungen in den Interviewverläufen immer wieder auftauchen. Den Beginn des Leitfadens markiert die bereits eingeführte Einstiegsfrage, die auf die Problematisierung von Jugendgewalt aus der jeweiligen Erfahrung der befragten Fachpersonen abzielt: »Jugendgewalt - was ist Ihrer Erfahrung nach das Problem?«. Es fällt ins Auge, dass die Befragten in ihren Antworten auf eine fachbereichsspezifische Erfahrungsebene zugunsten einer übergeordneten - gesellschaftskritischen - Positionierung verzichten. Die Einstiegsfrage stellt sich daher insofern als problematisch dar, als sich die befragten Fachpersonen kritisch - und teilweise in sehr emotionaler Weise - von ihr distanzieren und damit Narrationen über fachbereichsspezifische Erfahrungen außen vor bleiben. Andererseits zeigt sich, dass die Einstiegsfrage durchaus Interpretationsspielraum eröffnet und unterschiedliche Bearbeitungsweisen zulässt. Beispielsweise kann die Distanzierung von der Einstiegsfrage - in welcher Weise sie auch immer erfolgt - als Relativierung einer adressierten Expertise gelesen werden. Die Einstiegsfrage aktiviert also nicht nur eine bloß kritische Distanzierung, sondern gleichzeitig auch eine Reformulierung der eigenen Expertise. Diese Relativierung der zugeschriebenen Expertise ist ein Muster, das die geführten Interviews auszeichnet und ein wichtiger Gegenstand dieses Kapitels ist. Eine zentrale Frage, welche die methodische Diskussion anregt, ist folglich, inwiefern diese Relativierung dem Erhebungsinstrument und/oder dem Forschungsgegenstand geschuldet ist. Zunächst werden also die Einstiegspassagen derjenigen Interviews hinsichtlich ihrer Interaktionsdynamik untersucht, die mit dieser Einstiegsfrage beginnen. Anschließend werden diejenigen Interviews, die mit einer Erzählaufforderung beginnen, untersucht. Dieses Vorgehen ermöglicht einen Vergleich hinsichtlich des Umgangs mit gegenseitigen Adressierungsweisen bzw. der Interaktionsdynamik in Abhängigkeit von der Einstiegsfrage. Daraufhin werden die zentralen Ergebnisse dieser ersten Interpretationen, die für die primär themenspezifischen Interpretationen (4.1.2 und 4.1.3) relevant sind, zusammengefasst. Abschließend leitet eine methodische und theoretische Diskussion über zu den folgenden Kapiteln.

\section{Herr Unterwasser, Fachbereichsleiter}

Interviewerin: "Ja (.) also (.) ähm (.) wie gesagt ich interessier mich eben für verschiedene Experten und sehe Sie auch als Experte der einen ganz bestimmten Erfahrungsraum hat (.) ähm in seiner Tätigkeit und dass es is eben was mich interessiert (.) also Ihr Tätigkeitsbereich (.) wo sehen Sie die Probleme wo sehen Sie Lösungsmöglichkeiten und so weiter also mich interessieren eben weniger äh generelle Antworten sondern eher wie sehen Sie die diese Lage aus Ihrem spezifischen Erfahrungshorizont heraus. Genau." Herr Unterwasser: "In Bezug aufs Thema Jugendgwalt?" 
Interviewerin: "Genau (.) und des wäre einfach so mein erste Einstiegsfrage (.) ähm Jugendgwalt - wo sehen Sie oder wo ist Ihrer Meinung nach das Problem oder Ihrer Erfahrung nach das Problem?"

Herr Unterwasser: "Hmhm (.) ich bin (.) s vermuetlich isch jetzt noch witzig ich ha grad hüte Morgen $n$ Workshop gmacht für Gymnasiaschte vo der Kanti `Name der Schule ‘.) also ich chumm jetzt grad (.) des mach ich sonst nöd also ich schaff nöd mit Jugendliche selber ich han mini mini Fachlüt wo für Jugendliche da sind ((atmet tief ein)) ich schaff lieber mit Erwachsene $\uparrow$ (.) wo n es Problem und so sie von mir wend glöst ha und und nöd so gern mit Jugendliche wo quasi müehend mit mir schaffe s mach i nöd so gern ((atmet tief ein)) der Workshop mit der Kanti mach i aber gern das is so wie ebbes für mich au ebbes erfrischends und ich chann ich chann hüfig au so wie mini Modell oder mini Gedanken auf grob stellen wie denke junge Mannen über das es sind nur Mannen ja $\uparrow$ es sind wirklich (.) so um die siebezähne und ich find des immer ganz spannend. (1.5) So und ich han hüte Morgen wieder festgestellt dass für mich zwei (.) ähm (2.5) Persone relativ prägend gsi sind in mini Denkkonschtrukt (.) des einen is der Tätertherapeuth $0 \mathrm{e}$ lemann $\uparrow$ (.) chennet sie möglicherwis schon mit sini Modell s Hamburger Modell und sini Erchlärigs ähm (.) Versüech oder sine Konschrukt zum (.) zu Verbindig (.) Männerrolle und Aggressivität $\downarrow$. (1.5) Die find ich sehr hilfrich $\uparrow$ (1.5) det gfind ich mich au wieder in minere Biographie (1.5) und dazue ds Forschig vom Wassilis Kassis $\uparrow$ (1.5) dazue äh die die (1.5) für mich halt au nachvollziebar Forschigsalag wo ner versuecht hat use $z$ finde was muss ich a ne Mensch a ne männliche Mensch atue zwische Geburt und zwänzge (.) damit er dann mit zwänzge an usgwachsener Gwalttäter isch was muss da für (.) äh Biographiestatione duchlaufe halt welche Erfahrige münn sich da wiederholt ha. (2.5) Ähm das sind so die die Modell wo wo $n$ ich wirklich hilfrich find au zum ebbes erchläre oder zum en Standpunkt inäh (2.0) basierend auf dene Modell find ich no grundsätzlich (2.5) wichtig isch sind die Erwachsene des merk ich au in minere Praxis also (.) ich ich äh tue det wo ich mini Fachstell ähm (.) um Hilf gruefe wird bi bi bi gwaltätige Jugendliche a Schuele oder im öffentliche Ruum über ,Name einer Organisation، über d Jugendawaltschaft oder wie au immer (.) tun ich relativ schnell (.) versuche d Fokus z lege oder mini Uftraggeber dafür z gwünne dass mer mit de Erwachsene schafft also mit de Eltere mit de Lehrer (1.5) und ich mach wirklich au sehr positive Erfahrige mit dem also da hätts wirklich au so Präzedenzvorzeigefäll gäh wo ma au in de Medie verbrate händ (.) wo (.) schwer problematische Jugendliche also s Paradebispiel is (...)" (Zeilen 3-28)

Mit Blick auf die Interaktionssituation der der Einstiegspassage des Interviews mit Herrn Unterwasser fällt auf, dass der Befragte nicht direkt an die Einstiegsfrage anknüpft, sondern zunächst eigene Schwerpunkte darstellt. Dabei werden zunächst diejenigen Aspekte deutlich, die er aus seiner Perspektive bezüglich seines Fachbereichs für relevant setzt: Er distanziert sich von der Arbeit mit Jugendlichen, die nur »mühend mit ihm arbeiten«. Seine Fachstelle reagiere vielmehr auf Nachfragen anderer Organisationen und Institutionen - »seiner Auftraggeber« - und versuche, diese dazu zu gewinnen, mit Erwach- 
senen - den Eltern und Lehrern - zusammenzuarbeiten. Neben der Schilderung der Auftragssituation seiner Fachstelle ist zentral, dass deren Adressaten nur junge Männer sind. In Bezug auf wissenschaftliche Modelle versucht Herr Unterwasser, sich in die Perspektive junger Männer hineinzuversetzen und deren Situation biografisch nachzuvollziehen, wobei auch seine eigene Biografie als Deutungshintergrund fungiert. Mit dieser Einleitung distanziert er sich von der Einstiegsfrage und der ihm darin zugeschriebenen Expertenrolle in Bezug auf die Arbeit mit Jugendlichen. In der Auseinandersetzung mit wissenschaftlichen Modellen zur Entstehung von »männlicher« Jugendgewalt reformuliert er seinen Expertenstatus. Er setzt damit der Einstiegsfrage die eigenen Relevanzen und Fragen gegenüber. Dieses Muster der Distanzierung und Reformulierung zeigt sich in beinahe allen Interviews und wird durch die Einstiegsfrage begünstigt. Es wird im Folgenden als »Relativierung des zugeschriebenen Status einer Expertin bzw. eines Experten« bezeichnet. Herr Unterwasser wird durch die Interviewerin direkt als »Experte« für Jugendgewalt adressiert und aufgefordert, Jugendgewaltprävention aus seiner Perspektive oder Erfahrung heraus zu problematisieren. Daraufhin erzählt Herr Unterwasser, dass er gerade von einem Workshop mit einer Klasse einer Kantonsschule gekommen sei, was er als »witzig « bewertet. Diese Bewertung wird vor dem Hintergrund deutbar, dass er angibt, normalerweise nicht mit Jugendlichen zu arbeiten, sondern mit Erwachsenen. Sein Besuch der Kantonsschule stellt damit eigentlich eine Ausnahme dar. Die Arbeit mit Jugendlichen überlasse er seinen »Fachleuten «. Die Differenzierung zwischen den »Fachleuten«, die mit Jugendlichen arbeiten, und seiner Position als »Fachbereichsleiter«, der mit Erwachsenen bzw. Fachleuten arbeitet, beschreibt eine Hierarchie, was für die Reformulierung seiner Expertise relevant zu sein scheint. Indem er angibt, gerade an dem Tag des Interviews (im Rahmen dessen er als Experte für Jugendgewalt adressiert wird) auch tatsächlich mit Jugendlichen gearbeitet zu haben, unterstreicht er die Zufälligkeit und den Ausnahmestatus dieses Ereignisses. Indem Herr Unterwasser sich von der Arbeit mit Jugendlichen distanziert und dafür seine Expertise in der Arbeit mit Erwachsenen einbringt, relativiert er seinen Expertenstatus bezüglich Jugendgewaltprävention. Das, was er durch die Differenzierung zwischen der Arbeit mit Jugendlichen und Erwachsenen problematisiert, ist nicht Jugendgewalt, sondern die Arbeit mit Jugendlichen selbst, die den Jugendlichen Mühe bereite. Im Gegensatz zu den Erwachsenen, die konkrete problemlösungsorientierte Forderungen oder Bedürfnisse haben würden, würden Jugendliche zum Ausdruck bringen, dass ihnen die Arbeit mit ihm Mühe bereite, was wiederum ihm Mühe bereite. Seine Strategie, diese »Mühe « zu umgehen, könnte in der Aufwertung der Arbeit mit Erwachsenen bestehen. Dies beschreibt er jedoch vor seinen Auftraggebern sowie der Öffentlichkeit als hoch legitimationsbedürftig. Er müsse die Auftraggeber dazu »gewinnen«, mit Erwachsenen zu arbeiten (und eben nicht primär 
mit Jugendlichen). Dass Herr Unterwasser mit dieser Strategie »auch wirklich sehr positive Erfahrungen macht«, verdeutlicht nicht nur die Frage der Legitimität, sondern weist auch darauf hin, dass sein Erfolg nicht unbedingt selbstverständlich ist. Die mediale Inszenierung von »Präzedenzvorzeigefällen « sieht er zwar kritisch - sie hätten sie in den Medien verbraten -, lehnt sie jedoch vor dem Hintergrund eines gewissen Legitimations- bzw. Erfolgsdrucks nicht ab. Seinen Erzählungen über den Workshop in der Kantonsschule räumt Herr Unterwasser relativ viel Raum ein. In dieser Arbeit spielen wissenschaftliche Modelle eine große Rolle: Er sieht sich sowohl durch theoretisches Wissen als auch durch die Rezeption von Forschungsergebnissen dazu befähigt, Erklärungen für seine Beobachtungen zu liefern und einen »Standpunkt einzunehmen«. Er rekonstruiert seinen Expertenstatus bezüglich Jugendgewalt bzw. Jugendgewaltprävention also nicht nur über seine Leitungsfunktion, die ihm ermöglicht, mit Erwachsenen zu arbeiten, sondern auch über den Rekurs auf die Bedeutung wissenschaftlichen Wissens über die Entstehung von Jugendgewalt. Er sei dazu in der Lage, zu erklären und Stellung zu beziehen. Indem er fragt, was er einem männlichen Menschen antun müsse, damit er dann mit 20 ein ausgewachsener Gewalttäter sei, entwickelt er nicht nur die Idee einer »Täterkarriere« bzw. einer kriminellen Karriere, die im Erwachsenenalter beendet und irreversibel ist, sondern er weist den »betroffenen Tätern« gleichzeitig einen Opferstatus zu. Dies ermöglicht ihm auch, seine eigene Biografie sowie die Biografien anderer Erwachsener - u.a. derjenigen, mit denen er arbeitet -, zu erklären und nachzuvollziehen, wodurch er sein Wissen reproduziert. Er misst dem kriminologisch relevant gesetzten Wissen für die Beurteilung der (biografischen/sozialisationstheoretischen/psychologischen) Ursachen von Gewalt eine große Bedeutung bei und adressiert nichtkriminelle Erwachsene - nicht Jugendliche - als diejenigen Personen, die er einschätzen kann und mit denen er arbeiten kann. Die Vorstellung, dass »ausgewachsene Gewalttäter« ab dem Alter von 20 Jahren nicht mehr therapierbar seien, lässt darauf schließen, dass betreffende Maßnahmen, die diesen individuellen Karrieren entgegenwirken sollen, relativ früh beginnen müssten. Unter dieser Annahme wäre die Erfordernis, mit Kindern und Jugendlichen zu arbeiten, plausibel, was die Distanzierung von der Arbeit mit Kindern und Jugendlichen in einem paradoxen Licht erscheinen lässt. Herr Unterwasser deutet hier Gewalt als Resultat einer Erlebens- und Erduldungsbiografie, womit er (männlichen) Kindern und Jugendlichen eine gewisse Passivität unterstellt und den Blick auf deren »Opferrolle« als (potenzielle) Täter lenkt. Bemerkenswert ist außerdem, dass er aus dieser Perspektive nicht Jugendgewalt problematisiert, sondern »Erwachsenengewalt«, was seine Distanzierung in Bezug auf die Arbeit mit Jugendlichen wiederum plausibilisiert. Unklar bleibt hier zunächst, ob und wie aus seiner Perspektive den »Täterkarrieren« vorgebeugt werden kann bzw. welche Interventionsmöglichkeiten bestehen. Anzunehmen 
ist allerdings, dass Herrn Unterwassers Problemdeutungen stark von seiner Funktion als Fachbereichsleiter geprägt sind: Als solcher ist er in seinem Handeln abhängig von Aufträgen anderer Institutionen und Organisationen, die er »gewinnen« muss, und steht unter dem Druck, seine Arbeit als Erfolg zu inszenieren.

\section{Frau Schock, Familientherapeutin und Jugendarbeiterin}

Interviewerin: "Sie bieten ja auch ähm (2.0) im Bereich Gewaltprävention Sachen an hab ich gesehen machen da einiges (.) und ja da wollt ich einfach mal zum Einstieg (.) fragen (2.0) ähm Jugendgewalt wo sehen Sie das das Problem das Kernproblem und ja also ich möchte eben gerne (.) eben (.) so (.) von von Ihrem von Ihrem Ar also aus Ihrer Perspektive eben mit den Erfahrungen die sie jetzt da gemacht haben in ihrer Arbeit. (2.0) Genau! ((lacht))“

Frau Schock: "Ok wo ich den den Ur den Ursprung das Ursprung vom Problem anhand von meiner Erfahrung von meiner Arbeit. ((atmet tief ein)) Ok ich arbeite mit Familien $\uparrow$

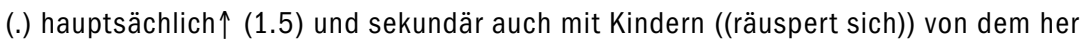
hab ich (.) die Perspektive in der Familie." (.) Und (.) jede Familie ist individuell das ist äh das ist so aber es gibt vielleicht Ähnlichkeiten oder Parallelen (.) bei der Erziehung der Kinder bei der Art und Weise Kinder zu erziehen. (2.0) Es tönt sehr pauschal ähm vielleicht ein Migrationshintergrund muss aber nicht sein ich kenn auch Schweizer Familien die (1.0) das Problem kennen (.) und ähm (3.5) die Art und Weise die diese Jugendlichen sehr wahrscheinlich groß geworden sind (.) plus eine ganz individuelle äh psychologische äh Struktur (.) das kommt auch dazu also das sind glaub ich (.) verschiedene Aspekte. (.) Sie kennen die soziologischen Aspekte ich sehe vielleicht mehr einen Blick in den Bindungen in den Beziehungen also vielleicht mehr das Psychologische und (.) das Pädagogische und da gibt's oft ähm (.) Parallelen (.) ohne jetzt grad zu pauschal sein zu wollen (.) ja."

Interviewerin: "Und wie sieht Ihre Arbeit mit den Familien aus was machen Sie da genau?"

Frau Schock: "Ähm also ich bin beauftragt meistens von Ämtern wie Jugendamt äh Gerichts äh Jugendgericht äh (.) und so weiter und es geht oft darum Eltern in ihrer Rolle als Eltern zu aktivieren es ist oft äh ähm ein Problem in der Beziehung zu den Kindern. (.) Kinder werden meistens in der Schule oder im Kindergarten auffällig $\uparrow$ und dann ist oft so (.) in der Schweiz ähm (1.5) das System die Schule ist sehr mächtig Kindergärten sind sehr mächtig und machen sehr viel Druck (.) auf Familie auf Behörde (.) und und drücken aus dass es mit den Kind so nicht geht $\uparrow$ (.) es braucht eine Familienbegleitung und dann löst sich alles weil die Familienbegleitung kommt mit einem magischen Stab und löst alles $\uparrow$ und so einfach geht auch nicht also es ist oft äh (.) jetzt äh ohne einen interkultulturellen oder Migrationshintergrund das Thema (.) wie kommunizieren Eltern zu ihren Kindern ist es eigentlich (.) einfacher als was man denkt es sind so auch kleine (.) ähm Kleinigkeiten die dann einem auffallen oder mir auffallen wo ich dann anknüpfe 
wo dann vielleicht mehr (.) mehr verbirgt aber eigentlich sind die Anfänge ganz klein.“ (Zeilen 1-26)

Die Frage nach dem Kernproblem von Jugendgewalt formuliert Frau Schock, die mit einem Pilotprojekt in der aufsuchenden Jugendarbeit wirbt und aufgrund dieser Werbung auch von der Forscherin für ein Interview angefragt worden ist, in die Frage nach dem »Ursprung « des Problems um und setzt somit eine andere Relevanz als die Forscherin. Anschließend beschreibt sie die Perspektive, aus der heraus sie den Ursprung des Problems beobachtet: die Perspektive der Familie. Sie situiert sich gleichzeitig in die Familie und außerhalb der Familie, indem sie angibt, primär mit ihnen zu arbeiten und sekundär mit Kindern - eine ambivalente Positionierung. Indem sie die Frage nach dem Problem der Jugendgewalt in die Frage nach dem Ursprung des Problems reformuliert und sich primär als Familientherapeutin, die in und mit Familien und Kindern arbeitet, darstellt, distanziert auch sie sich von der Adressierung als Expertin für Jugendgewalt. Anschließend führt sie verschiedene mögliche »Ursprünge« von Jugendgewalt und ihr Zusammenwirken auf. Sie stellt klar, dass ihr Blick von der Psychologie und der Pädagogik geleitet ist und misst damit dem wissenschaftlichen Wissen eine ähnliche Bedeutung bei wie Herr Unterwasser. Dabei grenzt sie das für sie relevante Wissen von dem »soziologischen « Wissen ab, über das ihrer Ansicht nach die Forscherin verfügt. Auch Frau Schock setzt die Strategie der Distanzierung dafür ein, ihre eigenen Relevanzen zu entwickeln: Sie sieht den Ursprung des Problems der Jugendgewalt in der Beziehung und Kommunikation zwischen Eltern und ihren Kindern. Diese Sichtweise kann sie gut mit der klaren Forderung ihrer Auftraggeberinnen bzw. Auftraggeber, mit den Eltern bzw. den Familien zu arbeiten, vereinbaren. Was sie im Kontext ihrer Arbeit problematisiert, ist nicht Jugendgewalt selbst, sondern die hohe Erwartungshaltung ihrer Auftraggeberinnen, die davon ausgehen, dass »die Familienbegleitung mit einem magischen Stab kommt und alles löst «. Dabei kritisiert sie den hohen Einfluss der Schweizer Erziehungs- und Bildungseinrichtungen, die über Maßnahmen entscheiden und den Erwartungsdruck letztlich auslösen würden. Frau Schock verdeutlicht, dass nicht sie mit den jeweiligen Problemen der Jugendgewalt konfrontiert sei, sondern die Erziehungs- und Bildungseinrichtungen, in denen Kinder auffällig werden. Frau Schock sei mit dem Auftrag konfrontiert, diejenigen Probleme zu lösen, die andere Institutionen (u.a. ihre Auftraggeberinnen bzw. Auftraggeber) relevant setzen, was mit einem hohen Erwartungsdruck einhergehe. Somit wird plausibel, dass Frau Schock den Ursprung des Problems relevant setzt und in der Familie verortet, weil sie so mit ihren Auftraggeberinnen bzw. Auftraggebern konform geht. Letztlich problematisiert sie jedoch eine strukturell bedingte Differenzierung zwischen dem Problem Jugendgewalt selbst und dem Ursprung des Problems. Das Problem selbst trete in den 
Erziehungs- und Bildungseinrichtungen auf, die den Ursprung in der Familie verorten, zu der sie aber keinen Zutritt haben. Frau Schock hingegen sei der Zutritt zu dem Problem selbst verwehrt. Somit sind die Erwartungshaltungen bezüglich der Problemlösung nur schwer miteinander zu vereinbaren, obgleich die verschiedenen Parteien dieselbe Problematisierungsweise zeigen. Auch Frau Schocks Problemdeutungen und Relevanzen sind also stark von ihren Auftraggeberinnen bzw. Auftraggebern geprägt. Dabei fällt auch auf, dass sie das Thema »Migration« problematisiert: Sie möchte zwar keine falsche Fährte legen, weist jedoch Familien mit »Migrationshintergrund « wiederholt einen besonderen Problemstatus zu.

\section{Herr Gold, Leiter einer privaten Organisation}

Interviewerin: "Wunderbar, dann können wir loslegen. (.) Nocheinmal vielen Dank dass Sie mich unterstützen und ich leg jetzt einfach gleich mal (.) los ähm und ich würd Sie noch mal äh (.) einfach bitten ich hab verschiedene Fragen (.) im Kopf $\uparrow$ die ich Ihnen stelle die mich interessieren $\uparrow$ und ähm äh ich bitte Sie einfach ganz ähm offen zu antworten was Ihnen (.) einfällt ich wird Sie dann auch nicht unterbrechen $\uparrow$ und ähm (.) ja es gibt auch kein Richtig oder Falsch es geht eben um Ihren Erfahrungshorizont zu dem Thema $\downarrow$. (1.0) Genau und genau. (.) Die erste Frage ist einfach ähm (.) Jugendgewalt was ist aus Ihrer Erfahrung nach das Problem?"

Herr Gold: "((lacht))"

Interviewerin: "((lacht laut))"

Herr Gold: "((lacht laut)) Oouu sehr eine heisse Frog ja ähm ich ich chann die nöd ich chann die also daaas Problem ich channs ned a so beantworte ganz ährlich gsait (.) ähm is für mi schwierig total s Problem uus z macha viellicht isch isch das Problem von Jugendgwalt mehr (.) mehr s ähm ähm s äh s s s s semantisch s Problem im sinn vo dass es immer wieder (.) thematisiert wird und und in der Gsellschaft schlussendlich immer wieder heißt: Hoho die hüütige Jugend ist so schlimm $\uparrow$ und (.) und das ghöre ma seit zwanzg Johr und werden ma wahrschinlich in zwanzg Johr wieder ghöre und des wird immer des Problem bliba wi ma sehr gern (.) eifach die allgemeinen Probleme der Gsellschaft a chli auf die Jugend projiziert und das uusmacht und (.) $\downarrow$ das Problem von Jugendgwalt seh $n$ iiich jetzt nöd s hätt sicher bstimmte Ursachen aber-« (Zeilen 1-19)

Herr Gold bedient sich auf die Frage nach dem Problem von Jugendgewalt des gesellschaftlichen Diskurses über Jugend und Gewalt kritisch, ohne aber konkret auf seine (fachbereichsspezifische) Erfahrung einzugehen. Er weist die Zuschreibungen der Interviewerin also deutlich zurück, insofern als er die Frage nach dem Problem von Jugendgewalt im Sinne einer Diskursreproduktion selbst problematisiert. Die Bezeichnung als »heiße Frage« in Verbindung mit einem Lachen kann sogar als ironische Distanzierung gelesen werden. Der zugeschriebenen Expertenrolle im Sinne einer Erfahrung im Kontext von Jugendgewaltprävention entzieht er sich und stellt sich zunächst 
als Gesellschaftskritiker dar. An seiner kritischen Haltung fällt eine gewisse Resignation hinsichtlich der dauerhaften Problematisierung von Jugendgewalt auf, an der sich seiner Prognose nach nichts ändern werde. Jugendgewalt liegt aus seiner Perspektive demnach primär als »semantisches Problem« vor, das er im Sinne einer historischen Konstante normalisiert. Darüber hinaus kann Jugendgewalt hinsichtlich seiner Ursachen betrachtet werden, was Herr Gold jedoch nicht weiter expliziert. Der Verzicht auf die Einbindung seines eigenen Erfahrungshorizonts sowie seine Ablehnung, Ursachen und Lösungsmöglichkeiten des Problems zu konkretisieren, können als Distanzierung von der Zuschreibung als Experte für Jugendgewaltprävention gedeutet werden. Er nutzt die Bühne, um eine allgemeine gesellschaftskritische Stellungnahme zu äußern, und stellt sich damit als Gesellschaftskritiker im Kontext von Jugendgewaltprävention dar.

\section{Herr Vonderstatt, Leitung einer städtischen Jugendarbeit}

Interviewerin: "Danke ((lacht)) (4.5) Wie gesagt, ich interessiere mich für das Thema Gewalt $\uparrow$ (.) Jugendgewalt und Gewalt (.) Jugendgewaltprävention ähm (.) und versuch des aus so aus soziologischer Perspektive zu fassen (.) und hab mir eben überlegt ich mach Interviews mit Experten (.) und mit Jugendlichen und versuch auch die Wahrnehmungsweisen einander gegenüberzustellen. Und ich interessier mich eben für Ihre (.) für (.) für die Sicht aus Ihrem Arbeitsbereich (.) heraus (.) auf dieses Problem. (1.5) Und ähm (.) zunächst würd ich einfach gerne wissen wie können Sie des auf den Kern bringen ähm was Jugendgewalt ähm was ist das Problem?"

Herr Vonderstatt: "Sie meinen die Gretchenfrage: Was ist des Problem überhaupt beim Thema Jugendgewalt?"

Interviewerin: "Hmhm."

B: "Äh (.) Jugendliche (2.0) in ihrer Entwicklungsphase meiner meiner Haltung nach meiner Meinung kommen in einem Zeitraum oder in einem in einem bestimmten Alter (.) bei welchem sie äh bei welchem es stark darum geht um Grenzen zu verletz oder auchzuloten oder äh (.) so das eigene Gebiet auch ein wenig klar umrissen darzustellen (.) in diesem äh (.) in diesem Zeitraum gibt es verschiedene Phänomene die zusätzlich dazukommen das sind Gruppenphänomene also die Peer Group die wichtig ist es gibt aber auch äh verschiedene Geschichten wie Perspektivenlosigkeiten und so weiter welche den Jugendlichen dazu verleiten kann Gewalt auszuüben. Jugendgewalt ist für mich ein Phänomen das kommt und geht (.) auf der einen Seite es kommt und geht in der Entwicklung der Menschen also in der Sozialisation (.) der Menschen $\uparrow$ es ist äh oft mal so da auch wenn wir die großen Zahlen beobachten dann ziehen die sich äh nicht ins Erwachsenenalter so weiter oder die welle die geht (.) nach unten (.) und äh es kommt und geht auch als phänomen in der öffentlichkeit in der wahrnehmung (.) Jugendgewalt äh ist heute nicht das gleiche wie vor 20 Jahren $\uparrow$ (.) da gab es ganz anderen Definitionen es auch ganz andere Hemmschwellen $\uparrow$ (.) äh und diese Hemmschwellen (.) die sind äh die sind eben komplett anders dadurch äh (.) bin ich auch der Meinung kann man Ju- 
gendgewalt von heute mit Jugendgewalt von früher vergleichen weil man von zwei oder drei verschiedenen Dingen spricht (.) das Problem (.) von Jugendgewalt das ist immer (.) die Balance zu finden zwischen was ist gesellschaftlich (.) vertretbar wie viel ist gesellschaftliche vertretbar und wie viel ist gesellschaftlich nicht mehr vertretbar. (.) Im Einzelfall ist Jugendgewalt nicht anders als Konflikte $\uparrow$ (.) und äh und in der Konfliktarbeit benötigen Jugendliche (.) Heranwachsende unter Umständen eine Unterstützung dass sie befähigt Konflikte zu lösen." (Zeilen 5-25)

Die Frage nach dem Problem von Jugendgewalt bezeichnet Herr Vonderstatt als »Gretchenfrage«, was darauf hinweist, dass er dieser Frage eine hohe Relevanz zumisst und/oder sie, ähnlich wie Herr Gold die »heiße Frage«, ironisch auffasst. Er setzt aus seiner »Haltung« bzw. »Meinung« heraus die »Entwicklungsphase« Jugendlicher relevant, um deren Handlungsweisen zu erklären. Diese Perspektive habe jedoch nicht die alleinige Erklärungskraft für das Handeln Jugendlicher. Er sieht in der »Peer Group«, der »Perspektivenlosigkeit« und der »öffentlichen Wahrnehmung« weitere Erklärungsfaktoren. Damit konstruiert er implizit eine Differenz zwischen »normaler« Jugendgewalt, die »kommt und geht« und problematisierbarer bzw. problematisierter Jugendgewalt, die »gesellschaftlich nicht mehr vertretbar ist«. Einerseits deutet Herr Vonderstatt Jugendgewalt als ein entwicklungspsychologisch erklärbares, »normales « Phänomen, das historisch gesehen unterschiedliche Formen annimmt. Andererseits entscheidet seiner Ansicht nach »die Gesellschaft« darüber, was dabei problematisch oder problematisierbar ist. Diese Differenzierung impliziert auch eine Unterscheidung zwischen »der Gesellschaft« und »der Jugend « bzw. »jugendlichem Handeln«. Jugendgewalt sei aus der Perspektive des »Einzelfalls« ein Zeichen dafür, dass der oder die Jugendlichen Hilfe bräuchten. Es fällt auf, dass Herr Vonderstatts »Haltung« bzw. »Meinung« stark von einer entwicklungspsychologisch versierten Perspektive auf Jugendliche geprägt ist. Damit setzt er, ähnlich wie Herr Unterwasser und Frau Schock, wissenschaftlich erzeugtes Wissen relevant. Er bezieht sich dabei jedoch, ähnlich wie Herr Gold, nicht auf seine fachbereichsspezifische Erfahrung, sondern bleibt allgemein. Darüber hinaus fällt auf, dass auch Herr Vonderstatt, ähnlich wie Herr Unterwasser, jugendlichen Täterinnen bzw. Tätern einen Opferstatus zuweist: Er stellt die Frage zentral, wie erklärt werden könne, was »den Jugendlichen dazu verleiten kann, Gewalt auszuüben«.

\section{Herr Tanner, Leiter der offenen Jugendarbeit einer Stadt}

Interviewerin: "Jetzt läufts!“

Herr Tanner: "Ok und ich lass es gleich beim äh Hochdeutsch (.) stehen. (.) Das ist das ist ein Reflex von mir ich hoffe Sie haben nichts dagegen."

Interviewerin: "Wie Sie sich wohler fühlen." 
Herr Tanner: "Genau dann ist für mich so am Besten weil wenn jemand Hochdeutsch spricht dann sprech ich direkt automatisch Hochdeutsch das hat mit meiner Geschichte zu tun."

Interviewerin: "Ok»

Herr Tanner: "So! ((lacht)) Ich ähm äh äh bin seit 25 Jahren in der Soziokultur habe zuerst 7 Jahre Jugendarbeit geleistet in einem Gemeinschaftszentrum (.) dann äh in der Quartier Erwachsenen Gemeinwesenarbeit (.) und dann das Gemeinschaftszentrum geleitet. (.) Das auch nochmal 7 nein sogar 9 jahre und seit äh jetzt ungefähr 15 Monaten bin ich hier als Geschäftsführer der Jugendarbeit Stadt ,Name der Stadtı das heißt ich bin seit ähm gut 18 Jahren nicht mehr als Jugendarbeiter tätig (.) ähm war allerdings im Gemeinschaftszentrum immer nahe an der Jugendarbeit also es war ein ein Bereich der integriert war im im Zentrum es waren nicht einfach zwei Jugendarbeiterinnen die da zuständig waren sondern wir haben des immer als Ganzheitliches äh angeschaut ähm ich hatte oft die Rolle des Bösen $\uparrow$ (.) also dann wenn es äh zu zu interventionen kam dann kam ich $\uparrow$ (.) und (.) wenn es wieder darum ging Beziehungsarbeit zu leisten mit den Jugendlichen ( ) Wiedergutmachung ging das war dann wieder Aufgabe der Jugendarbeiterinnen wobei wir also zusammen aufgetreten sind (.) aber quasi so diese (.) Rollenteilung hatte das funktionierte aber nur $\uparrow$ (.) wenn ich (.) zu den Jugendlichen schon Kontakt hatte $\downarrow$ oder $\uparrow$ wenn ich wenn keine Beziehung da war dann war das praktisch nicht möglich also war ich immer wieder ein bisschen präsent ich ich ich ließ mich sehen äh ich habe auch Betreuungszeiten übernommen damit die Jugendlichen mich immer kannten insbesondere wenn Generationenwechsel und immer neue Jugendliche dazu kamen so. (.) Jetzt bin ich ganz weit weg von den Jugendlichen außer dass ich vielleicht zwei jugendliche äh Kinder habe $\uparrow$ (.) ähm und kann aktuell eigentlich nur vom Hören Sagen oder von den Schilderungen oder von den Diskussion die ich mit meinen stellenleitenden Mitarbeiterinnen habe so."

Interviewerin: "Ok (.) schön und war des auch alles immer hier in 'Name der Stadt (.) so Ihre Laufbaun Laufbahn?"

Herr Tanner: "Das war immer in der Stadt ,Name der Stadt، ja"

Interviewerin: "Hmhm (.) schön! (2.0) Ich bin eben wie gesagt in meinen Recherchen ähem eben immer wieder auf dieses Thema Jugendgewalt gestoßen weil das so präsent ist und ähm was würden Sie denn sagen wie bringen Sie des auf den Kern Jugendgewalt was ist das Problem das zentrale Problem? ((lacht))"

Herr Tanner: "Ähm (.) das weich ich gleich aus. (.) Ich weiß nicht ob das soziologisch ist oder nicht (.) ich find es ich find es bereits schon wenn ichs wenn ichs wirklich zugespitzt sage (.) find ich das dieser Ausdruck $\uparrow$ (.) ist eigentlich Gewalt (.) an die Jugend (.) oder? (.) Weil (.) es stimmt (.) dass man von Jugendgewalt reden kann (.) wie man von Kindergewalt reden kann oder von Seniorinnengewalt oder von Erwachsenen oder von Frauen oder von Männer oder was auch immer und dass man aber von Jugendgewalt und praktisch nur ausschließlich von Jugendgewalt spricht und sonst von Gewalt (.) beziehungsweise gar nicht sondern nur von Jugendgewalt (.) ist eigentlich Gewalt an die Jugend. (.) So! (.) Und mit dem möchte ich wirklich äh für mich auch sagen (.) ähm (.) 
es geht für mich eigentlich nicht an beziehungsweise die Gesellschaft macht sichs sehr einfach von Jugendgewalt zu sprechen ohne die anderen Facette Facetten oder Gewalt generell zu thematisieren (.) in der Gesellschaft (.) so!" (Zeilen 1-40)

Herr Tanner bricht mit der Erwartung an ein Interview als Frage-AntwortInteraktion, indem er eine mögliche Einstiegsfrage gar nicht erst abwartet, sondern von sich aus zu erzählen beginnt. Er schreibt sich somit selbst die Rolle zu, das Interview zu eröffnen sowie die Interviewsprache festzulegen. Er besteht darauf, das Interview auf Hochdeutsch zu führen, und begründet das biografisch. Das Thema seiner Biografie verfolgt er weiter, was dafür spricht, dass es für ihn im Kontext dieses Interviews einen zentralen Stellenwert einnimmt. Anhand der Schilderung seiner Biografie distanziert er sich von dem Anspruch, aktuell aus einer den jugendlichen Lebenswelten nahen Perspektive zu sprechen. In seiner leitenden Funktion nehme die Arbeit mit Jugendlichen keinen hohen Stellenwert ein. Anhand seiner weit zurückliegenden Erfahrung als Jugendarbeiter definiert er Jugendarbeit als »Beziehungsarbeit«. Damals habe er die Rolle des »Bösen« eingenommen, für die der unmittelbare Kontakt zu den Jugendlichen notwendig gewesen sei. Von der mit dem Interview einhergehenden Adressierung als Experte für Jugendgewaltprävention distanziert er sich also zunächst biografisch. Dabei führt er eine Unterscheidung ein, die sich im weiteren Interviewverlauf noch als bedeutend erweist: Die Unterscheidung von Intervention und Prävention. Intervention sei der Rolle des »Bösen« angemessen. Die »Jugendarbeiterinnen« hätten es dann »wieder gut gemacht«. Auf diese Distanzierung von Jugendarbeit folgt die Frage der Interviewerin nach dem zentralen Problem von Jugendgewalt - die eigentliche Einstiegsfrage. An diese Frage schließt Herr Tanner, ähnlich wie Herr Gold, insgesamt gesellschaftskritisch an und ist bestrebt, eine soziologische Perspektive einzunehmen. Damit erweitert er seine Distanzierung von der Adressierung als »Experte« für Jugendgewaltprävention: Er setzt weniger sein eigenes, fachbereichsspezifisches Wissen als vielmehr »soziologisches« Wissen als den Maßstab, um angemessen über diese Thematik zu sprechen. Ähnlich wie Herr Gold problematisiert er Jugendgewalt auf semantischer Ebene. Gewalt müsse differenziert werden bezüglich der Personengruppen, die Gewalt ausübten. Nur dann sei es legitim, auch über Jugendgewalt zu sprechen. Alles andere sei »Gewalt an der Jugend«. Ähnlich wie Herr Gold nutzt er die Plattform des Interviews zunächst weniger dazu, aus seinem fachbereichsspezifischen Erfahrungshorizont heraus zu sprechen, stattdessen äußert er Gesellschaftskritik. Die Interpretationen werden noch zeigen, dass die Befragten ihre Perspektiven aushandeln und zwischen unterschiedlichen Positionen (Gesellschaftskritik, Biografie und Praxis etc.) oszillieren. 
Die bisherigen Interpretationen zeigen, dass die gewählte Einstiegsfrage unter Umständen zu gewissen Irritationen führt, insofern sich die Befragten mehr oder weniger stark von ihr distanzieren. Herr Gold und Herr Vonderstatt ironisieren die Frage sogar. Herr Gold und Herr Tanner nehmen diese Frage zum Anlass einer kritischen Reflexion über den Begriff Jugendgewalt. Da diese Frage ihr eigentliches Ziel, Erzählungen bezüglich der fachbereichsspezifischen Problematisierungs- und Lösungsweisen $\mathrm{zu}$ aktivieren, $\mathrm{zu}$ verfehlen drohte, entschied sich die Forscherin dafür, die Einstiegsfrage während des Erhebungsprozesses zu ändern und eine Erzählaufforderung zum beruflichen Alltag an den Beginn des Leitfadens zu platzieren. Folgende Interpretationen betreffen nun die Einstiegspassagen derjenigen Interviews, die mit einer offenen Erzählaufforderung beginnen, was diesbezügliche Vergleiche anregt und die Sensibilität für den Einsatz von »Experteninterviews « im Rahmen diskursanalytischer Forschungsarbeiten erhöht. Die Befragten werden mittels der offenen Erzählaufforderung nicht mehr primär als Expertinnen bzw. Experten für Jugendgewalt adressiert, insoweit sie dazu aufgefordert werden, über das Kernproblem zu sprechen. Sondern sie werden dazu aufgefordert, zunächst offen über ihre Arbeit zu erzählen, mit dem Ziel, spezifische Nachfragen zu Problematisierungsweisen von Jugendgewalt sowie Präventionskonzepten zu ermöglichen. Dabei muss berücksichtigt werden, dass die Befragten auch durch die Rekrutierung in Form des Anschreibens in gewisser Weise als Expertinnen bzw. Experten von Jugendgewalt adressiert sind - ihre Erwartungshaltung also davon geprägt ist. Daher wird der Beobachtung einer Relativierung des durch die Forscherin zugeschriebenen »Expertenstatus« weiter Rechnung getragen: Ist diese Relativierung eine Strategie, die etwas über das Feld »Jugendgewaltprävention« und seine Spezifik aussagt, oder ist sie eine Strategie, die etwas über das Erhebungsinstrument aussagt? Des Weiteren gibt der Vergleich der Interpretationen unterschiedlicher Einstiege Aufschluss über Möglichkeiten der Rekonstruierbarkeit gemeinsamer Deutungsmuster im Sinne eines methodischen Vergleichs, der reflexiv auf die Rekonstruktion immanenten Wissens über Jugendgewalt und Jugendgewaltprävention abzielt, die in einem nächsten Analyseschritt verfolgt wird (4.1.2): Wie positionieren sich die Befragten und welche Relevanzen bezüglich Jugendgewalt und Jugendgewaltprävention legen sie damit fest?

\section{Frau Blaum, Jugendarbeiterin}

Interviewerin: "Gut also vielen Dank dass Sie mir des Interview ermöglicht haben. (.) Ich wollte ganz einfach am Anfang einmal ähm (.) einfach von Ihnen erfahren wie Ihre Arbeit hier aussieht mit den Jugendlichen so im Alltag was was machen Sie eigentlich worin besteht Ihre Tätigkeit in der ,Name der Organisation`?"

Frau Blaum: "Äh also unser Hauptangebot ist eigentlich unsre Öffnungszeiten $\uparrow$ (.) wo die Jugendlichen freiwillig kommen können (.) und und es geht in dem Bereich um Frei- 
zeitbeschäftigung $\uparrow$. (1.5) Ääh sie können einfach da sein sie können mit den anderen Jugendlichen in Kontakt treten sie können mit uns sprechen sie können auch spielen was auch immer äähm (1.5) wir tun dann zusammen kochen also sie müssen einfach einen Bedarf erklären was sie machen möchten und dann werden wir aktiv (.) dass wir diese Freizeit so (.) zusammen verbringen. (.) Äähm (.) dann haben wir spezifische Angebote äh (.) zum Beispiel für Mädchen (.) äähm (.) weil (.) im Quartier haben wir Mädchen wo sehr nicht in den normalen Treff während der normalen Öffnungszeiten hierherkommen dürfen. (1.5) Das sind Familien mit äh Migrations (.) Hintergrund und sehr wahrscheinlich muslimische Familien nehm ich an. (2.5) Ääh dann haben wir eben spezielle Öffnungszeiten für Mädchen $\uparrow$. (1.5) Und dann haben wir aber auch ein Mädchentreff wo Mädchen sich zurückziehen können und und und wenn sie allein unter sich sein möchten (.) einfach auch diese Möglichkeit haben. (.) Und beim Mädchentreff äh sind nur Mädchen da und dann machen wir mit denen (.) Aktivitäten. (2.5) Dann haben wir (.) 'Name der Veranstaltung، äh (.) das ist Samstag hier in einem Schulhaus die Hallen benützen können (.) äh wo die Jugendlichen (.) von acht bis elf Uhr einfach Sport machen können es ist alles freiwillig natürlich oder ohne Anmeldung ohne ( ) einfach. (1.5) Ääh das ist so die Hauptschiene und dann haben wir äh natürlich Beratungsangebote wo wir dann die Jugendlichen beraten begleiten wenn sie Lehrstellen suchen wenn die Arbeit suchen wenn sie Bewerbungen schreiben äh möchten äähm. (1.5) Aber auch sonst äh sei es Suchtproblematik sei es familiäre Probleme einfach dass wir da sie beraten aber auch weitervermitteln an die richtige Stelle äh. (2.0) Die dritte Schiene ist äh Projektarbeit wir sind gut vernetzt mit anderen Einrichtungen im Quartier die in dem Bereich tätig sind äh (.) äh wir führen dann gemeinsam Projekte durch aber auch mit Jugendlichen also (2.0) ohne Vernetzung (.) äh da geht es darum dass die Jugendliche ähm (1.0) etwas auf die Beine stellen möchten äh und wir tun sie dabei unterstützen. (3.5) Ja (1.5) das sind so die Haupt Hauptgebiete wo wir tätig sind ja“

Interviewerin: "Ok und wie ist des entschieden worden dass es diese Hauptgebiete gibt wie hat sich des entwickelt?"

Frau Blaum: "Es ist so äh (.) wir machen städtische Jugendarbeit und wir haben eine Leistungsvereinbarung äh mit der Stadt (.) wo die Stadt uns finanziert (.) und in dieser Leistungsvereinbarung sind klare Aufträge was wir (.) in welchen Bereichen wir tätig sein sollen und welche Bereiche wir abdecken sollen das wird vorgegeben äh vom Sozialdepartement. (.) Hmhm (.) Äh und dann dann tun wir einfach diese Aufträge zu erfüllen also versuche zu erfüllen ja klar und dann wir des immer Ende Jahr (.) ähm mit einem Reportinggespräch äh eben die Zahlen die Angebote Projekte Veranstaltungen einfach vorgelegt und und dann (.) überprüft."

Interviewerin: "Ah ok (.) und des gilt für alle (.) äh"

Frau Blaum: "Einrichtungen von 'Name der Organisation، ja klar ähä hmhm."

Interviewerin: "Und können Sie sagen welche Rolle spielt da ähm spielt das Gewaltprävention Jugendgewaltprävention (1.5) in der Arbeit?"

Frau Blaum: "Also (2.5) es ist auch ein Teil ein Auftrag (.) aber wir werden ja eher aktiv wenn Gewalt da ist dass unsere Schwierigkeit eben äh. (1.0) Wir können die Eltern nicht 
erreichen (1.0) wir wissen die familiäre Konschtellation nicht wir wissen auch die Hintergründe nicht. (.) Gewalt ist für uns ein Thema sobald sie da ist. (.) Hmhm äh und dann ist äh ist eigentlich zu spät präventiv noch was zu machen oder wir können höchstens die Jugendlichen sensibilisieren mit denen ein Gespräch führen (.) aber (.) tiefer können wir nicht gehen. (.) Die Jugendlichen sind freiwillig da es ist keine Verbindlichkeit da (.) und und und es gibt Jugendliche die nur ein Monat bei uns sind und nachher verschwinden und nachher hören wir von denen nichts also wir können sie nicht irgendwie nachhaltig lange begleiten (.) und von daher ist das schwierig für uns äh in dem Bereich wirklich nachhaltig äh (.) tätig zu sein ähä (.) ähä." (Zeilen 1-39)

Die offene Frage nach dem beruflichen Alltag als Jugendarbeiterin in der offenen Jugendarbeit ermöglicht Frau Blaum in einem Dreischritt vom »Sein« über »Können« hin zum »Müssen« die Konstruktion eines Raums, indem sie den Ist-Zustand offener Jugendarbeit zu einem Idealzustand entwickelt, der darin besteht, »Freizeit so zusammen zu verbringen«. Die Rahmenbedingungen offener Jugendarbeit zeichnen sich insbesondere durch die Freiwilligkeit der Teilnahme Jugendlicher aus, die über eine klare Regelung der Öffnungszeiten gesteuert ist, d.h., sie können sich ohne Ankündigung zu den vorgegebenen Zeiten in die Einrichtung begeben und finden dort stets bestimme Regelmäßigkeiten vor wie etwa Personen, mit denen sie sprechen können, und Ressourcen, die gemeinsame Aktivitäten wie etwa das Kochen gewährleisten. Kern und Ideal von Frau Blaums Tätigkeit, eben »Freizeit so zusammen zu verbringen«, scheint nur unter diesen Rahmenbedingungen realisierbar zu sein. Diesem Moment könne sich nur dann angenähert werden, wenn die Jugendlichen ihre eigenen Bedürfnisse zum Ausdruck brächten. Das Äußern eigener Wünsche der gemeinsamen Freizeitgestaltung durch die Jugendlichen und deren Realisierung durch die Jugendarbeiterinnen und Jugendarbeiter als Reaktion darauf kommen in dieser Darstellung als erfüllter Zustand offener Jugendarbeit in den Blick. Gemeinsame Aktivitäten müssten durch die Ansprüche der Jugendlichen initiiert werden. Die Haltung der Jugendarbeiterinnen und Jugendarbeiter mutet den Beschreibungen nach eher reaktiv an, insofern ihnen eine Erwartungshaltung zugeschrieben wird. Das »Konzept« der Freiwilligkeit fungiert als primäre Rahmenbedingung, die auch räumlich und zeitlich verankert ist. Neben den normalen Öffnungszeiten gebe es spezielle Öffnungszeiten für Mädchen, womit der Jugendtreff auf die Nachfrage der im Quartier lebenden Eltern bzw. Familien, die nicht möchten, dass ihre Töchter während der normalen Öffnungszeiten mit Buben zusammentreffen, reagiert. Frau Blaum geht davon aus, dass es kulturelle (»Migrationshintergrund «) oder religiöse Motive (»muslimische Familien«) sind, die Familien dazu bewegen, ihren Töchtern den Besuch zu normalen Öffnungszeiten zu verbieten. Neben den normalen Öffnungszeiten und den speziellen Öffnungszeiten für Mädchen gebe es noch den Mädchentreff, womit die Einrichtung wieder auf die 
Nachfrage der Mädchen, die das Zusammensein mit Mädchen bevorzugten, reagiert. Ein weiteres Hauptangebot bestehe in der Nutzungsmöglichkeit einer Schulsporthalle, was nach den gleichen Regeln organisiert sei wie die normalen Öffnungszeiten, also nach festgelegten Öffnungszeiten, auf freiwilliger Basis und ohne Anmeldung. Die Jugendlichen könnten in diesen Zeiten selbstständig Sport machen. Außerdem schildert Frau Blaum, die Jugendarbeiterinnen und Jugendarbeiter würden Aufgaben in der Beratung und Vermittlung von Jugendlichen wahrnehmen, die auf Arbeitssuche oder Lehrstellensuche seien oder Sucht- oder familiäre Probleme hätten. Als drittes Hauptangebot nennt Frau Blaum Projektarbeiten mit Jugendlichen und/oder mit anderen Institutionen. Dabei sei es an den Jugendlichen, zu entscheiden, welche Inhalte thematisiert werden sollen. Das Angebotsspektrum dieser Einrichtung wirkt, den Beschreibungen Frau Blaums nach zu schließen, sehr differenziert und an der Nachfrage der im Quartier lebenden Familien und Jugendlichen ausgerichtet. Was die Jugendarbeiterinnen und Jugendarbeiter konkret tun, expliziert Frau Blaum weniger. Vielmehr beschreibt sie eine Art Anpassung der institutionellen und räumlichen Rahmenbedingungen ihrer Einrichtung an die Nachfragen von außen. Die Jugendlichen könnten die Angebote der Einrichtung freiwillig und spontan nutzen und seien dazu angehalten, ihre Bedürfnisse zu artikulieren. Sie würden von außen in die Institution kommen und ihre Bedürfnisse mit einbringen. Die Haltung der Jugendarbeiterinnen und Jugendarbeiter mutet dabei insgesamt eher reaktiv an, womit Frau Blaum ihr in der vorhergehenden Sequenz geschildertes Ideal offener Jugendarbeit, auf die Ansprüche der Jugendlichen zu reagieren, reproduziert. Trotzdem versteht Frau Blaum sich und ihre Kolleginnen bzw. Kollegen als »aktiv« und »Aktivitäten machend «. Sie beschreibt auf Nachfrage hin ein weiteres Ideal offener Jugendarbeit, das in der Erfüllung eines Auftrags der Stadt besteht, einer »Leistungsvereinbarung «, der sie sich verpflichtet sieht, deren Realisierung sie jedoch infrage stellt. Gewaltprävention sei ein Teil des Auftrags, den die Freizeiteinrichtung von der Stadt, die sie finanziert, bekommen habe. Sie sieht sich mit dem Bedürfnis konfrontiert, gleichzeitig auf die Nachfrage der im Quartier lebenden Familien und Jugendlichen zu reagieren und die durch die Stadt formulierten Aufträge zu erfüllen. Dies beschreibt sie, wie folgt, als Dilemma: Auf die Nachfrage der Interviewerin, welche Rolle Jugendgewalt dabei spiele, äußert Frau Blaum Zweifel an der Realisierung des »Präventionsauftrags «, insofern sie erst dann tätig werden würde, »wenn Gewalt da ist« bzw. »sobald sie da ist«, was den reaktiven Charakter ihrer Arbeit unterstreicht und ein auf Vorbeugung und Nachhaltigkeit abzielendes Verständnis von Prävention beschreibt. Diese Art von Prävention sei nicht realisierbar, da die Strukturen offener Jugendarbeit, wie etwa Freiwilligkeit und die Unmöglichkeit, Eltern oder andere Hintergründe der Jugendlichen miteinzubeziehen, restriktiv wirkten. Die Nachfrage der im Quartier lebenden Familien und Jugendlichen wider- 
spricht der Nachfrage der Auftraggeberin, der Stadt. Frau Blaum steht dabei in dem Dilemma, beiden Aufträgen gerecht werden zu wollen bzw. zu sollen. Gewalt tritt aus ihrer Perspektive als Phänomen auf, dem gegenüber sich die Jugendarbeiterinnen und Jugendarbeiter ausschließlich reaktiv, nicht aber vorbeugend »verhalten« können. In dieser Hinsicht wird Prävention als Konzept oder gar konkrete Handlung gedeutet, die vorbeugt. Begründet wird diese Haltung über die die offene Jugendarbeit primär strukturierende Situation der Freiwilligkeit, die sich auf den konkreten Ort der Einrichtung konzentriert. Darüber hinaus wirken die Jugendarbeiterinnen und Jugendarbeiter in den Beschreibungen Frau Blaums weder aktiv noch reaktiv handlungsfähig, was Gewaltprävention, deren zentrales Charakteristikum die Nachhaltigkeit sei, zu verunmöglichen scheint. Die reaktive Wirkmächtigkeit der Jugendarbeiterinnen und Jugendarbeiter beschränkt sich auf einen klar abgegrenzten Raum, aus dem heraus auch das Wissen über die Familien der Jugendlichen, das für Prävention als zentral erachtet wird, nicht verfügbar ist. Die hier dargestellte »Handlungsohnmacht« bezüglich Gewaltprävention bricht mit dem Auftrag der Stadt, präventive Arbeit zu leisten. Frau Blaum, die offen dazu aufgefordert wird, über ihre Arbeit zu erzählen, hat die Möglichkeit, ihren Status als Expertin, ihre Problematisierungsweisen sowie Deutungskonstruktionen zu entwickeln, ohne zunächst womöglich provokativ wahrgenommene Zuschreibungen zu korrigieren. Dabei sticht hervor, dass sie aus dieser Offenheit heraus ihre Expertise idealisiert und vor dem Hintergrund dieser Idealisierung den Präventionsauftrag, den sie direkt von der Stadt erhält, problematisiert. Sie sieht ihre Expertise darin, ihr ideales Ziel, zusammen Freizeit zu verbringen, zu realisieren, was nur in einem Raum ermöglicht werden kann, in dem die Anwesenheit Jugendlicher freiwillig ist. Die Realisierung von Prävention, die sie als vorbeugende Handlung versteht, bricht mit ihrer Idealvorstellung von offener Jugendarbeit und den Rahmenbedingungen derselben. Frau Blaum relativiert ihre Expertise auf andere Weise: Sie gibt den Jugendlichen einen Teil davon ab. Der Idealzustand offener Jugendarbeit erfordert nicht nur eine »Professionalisierung « der Jugendarbeiterinnen und Jugendarbeiter, sondern auch der Jugendlichen selbst und zwar hinsichtlich ihrer Kompetenz, eigenen Bedürfnissen Ausdruck zu verleihen. Die Verantwortungsübernahme, welche die »Profession« offener Jugendarbeit aus der Perspektive Frau Blaums im Erreichen ihrer Ziele mit sich bringt, erwartet sie auch von ihren Klientinnen und Klienten.

\section{Herr Auto, Geschäftsführer einer privaten Organisation}

Interviewerin: "Ja grüezi Herr Auto jetzt bin ichs wieder die Verena Kuglstatter. (.) Ich hab jetzt ne Möglichkeit gefunden ähm wie viel Zeit haben Sie denn jetzt noch zu sprechen (9.0) Ja (.) also ne Halbe bis eine Stunde es kommt auch drau an wie viel Sie (.) erzählen oder wie viel Sie zu sagen haben ((lacht)) ja also wenns nicht anders geht würd ichs jetzt 
am (.) Telefon machen und so (.) ja (.) gut. (5.0) Also des Thema (.) ähm ich intressier mich für Jugendgewalt und Jugendgewaltprävention."

Herr Auto: "Hallo?"

Interviewerin: "Hören Sie mich noch (.) hören sie mich jetzt?"

Herr Auto: "Jetzt hör ich sie."

Interviewerin: „Entschuldigung ((lacht)) also es geht um Jugendgewalt und ähm Jugendgewaltprävention und Intervention ich bin soziologischen Institut und ich intressier mich für diejenigen Experten die im Bereich der Prävention und Intervention arbeiten und da eben (.) bestimmte Erfahrungen gemacht haben mit der Arbeit mit Jugendlichen oder mit Eltern oder mit Schülern oder mit Lehrern (.) und ich interessiert mich ähm (.) ja genau was des Problem is und warum auch in der Schweiz aben auch so viel darüber diskutiert wird (.) und ähm genau (.) und Sie sind (.) ja vielleicht können Sie einfach mal beschreiben was was Ihr Tätigkeitsbereich ist (.) was Sie eigentlich machen in dem Bereich!“

Herr Auto: "Ja (.) also unser Institut äh hat sich zum Ziel gesetzt dass Volksschulen egal mit welchem Problem in der Gewaltprävention oder Intervion zu uns kommen können und und ( ) äh Fachmitarbeiter die alle aus einem anderen Bereich der Gewaltprävention kommen es ist ist ja so in der Schweiz gibt's ja Fachspezialisten und wenig Generalisten."

Interviewerin: "Ok."

Herr Auto: "Und äh darum (.) kann man sich so gut zusammensetzen indem sich wie Bausteine oder? Derzeit haben wir 200 Institutionen etwa mit denen wir zusammenarbeiten die auch im Bereich der Gewaltprävention äh spezialisiert sind irgendwo (.) und dann haben wir im moment etwa 10 regionale während wir aber auf 30 aufstocken damit wir wirklich nahe bei den Schulen sind und ich selber ich äh (.) habe (.) ich mache schon schon eta 15 jahre lang ( ) Gewaltprävention (.) und schon äh hab ich mich jetzt einfach laufend laufend weitergebildet also ( ) vielen Spezialisten auch Weiterbildungen gemachten und (.) und hab mich halt so zum Generalisten ausgebildet." (Zeilen 1-23)

Herr Auto wird einleitend direkt als Experte für Jugendgewalt adressiert, schließlich aber dazu aufgefordert, allgemein seinen Tätigkeitsbereich zu beschreiben. Dabei spricht er zunächst aus der Perspektive der Organisation, die er leitet. Er beschreibt diese als dienstleistungsorientierte Organisation, die Spezialisten an Schulen vermittelt, wenn diese sich mit Problemen bei seiner Organisation melden. Seine Organisation fülle als »Generalistin« quasi eine Marktlücke. Daran entwickelt Herr Auto auch seine biografisch gewachsene Expertise als »Generalist«. Die Ziele und Inhalte seines Tätigkeitsbereiches muten ökonomisch oder zumindest in erheblicher Weise dienstleistungsorientiert an, insofern seine Organisation auf Nachfragen reagiert. Die jeweils für die Schulen spezifischen Probleme sind demnach auch nicht selbstverständlich präventiver Art, können ebenso interventiver oder anderer Art sein, was die ökonomische Begründung der Ziele und Aufgaben seiner Organisation 
weiter plausibilisiert. In dieser Hinsicht besteht seine Expertise als Generalist weniger in konkreter Gewaltprävention und vielmehr in der Vermittlung von Nachfrage und Angebot. Prävention wie auch Intervention legitimiert sich aus dieser Perspektive über ein Bedürfnis, das auf einem Markt in Form einer Nachfrage artikuliert wird.

\section{Herr Ifon, Leiter der Jugendpolizei einer Stadt}

Interviewerin: "Ich legs jetzt einfach mal dahin (.) so (.) genau. (.) Dann wär ich froh wenn Sie mir das jetzt noch kurz erklären ((lacht))."

Herr Ifon: "Also ( ) zu meiner Person Herr Ifon (.) 54 (.) seit 34 Jahr im Polizeidienscht (.) seit zweitusig ähm äh stellvertretender Leiter Jugenddienscht (.) seit zweitusivier (.) Leiter vom Jugenddienscht (.) von miner 34 Poliz äh ah Polizeijahr sind 24 Kriminalpolizei (.) der Rescht is an für sich (.) zuerscht Streifedienst gsi (.) ja (.) bin verhüratet aber keine Kinder. (.) Und wenn ich da mini Tätigkeit gseh bin ich eigentlich froh dass ich (.) keine Chind ha."

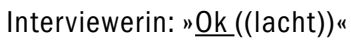

Herr Ifon: "Dass ich chlar nein (.) so viel zum und bin noch politisch tätig. (.) Ähm der Jugenddienscht vo der Stadtpolizei ,Name der Stadt، is $n$ reiner Ermittlungsdienst (.) also wenn ich Prävention isch bi eus (.) nöd im Hauptfokus (.) isch mir sind zu 75 \% sind wir Ermittler mir versueche use zfinde welche (.) Jugendliche händ (.) Straftate begange $\uparrow$ (.) ähm (.) spannend bei eus Jugenddienscht (.) dass d Arbeit generiert wird aufgrund vom Alter (.) vom (.) Beschuldigte. (.) Unter 18 durch das bewege mir eus im ganze Strafgsetzbuech. (.) Mir sind ja alle Nebegsetz Betäubungsmittelhandel Usländergsetz und so witer sind mir tätig (.) ähm im Gägäsatz zu (.) der Erwachsenenkriminalität wos n spezialisierte Gruppe gibt nur Einbruch oder nur Fahrzeugdelikte (.) mir machet wirklich alles-“ ((Das Gespräch findet in Herrn Ifons Büro statt wird kurz von einem seiner Kollegen unterbrochen, der an der Tür, die offen steht, stehenbleibt und Herrn Ifon etwas fragt. Sie unterhalten sich kurz.)) "Also (.) das ist der große Vorteil an sich durch das dass ebbe ( ) sehr wenig Fluktuation (.) ich ha gsaigt $75 \%$ eErmittlig (.) $25 \%$ isch Patroullientätigkeit (.) ähm des heißt mir sind uf der Gass in Zivil^ (.) aber eher ned als Prävention (.) sondern eifach (.) Präsenz Hotspots nagah (.) mit de Jugendliche Gspräch führe wo ma ja sehr hüfig da in euser ( ) chenne $\uparrow$ (.) und dann wüsse mir der 'Namer da isch ja $\mathrm{n}$ Räuber $\uparrow$ ah der isch ja mit däm und däm zamma und da chömme mehr so gwüsse (.) Infos über wer wo verchehrt und so witer. (.) Ähm äh s dritte Standbein wo mer händ is de starke Vernetzig mit de Behördene $\uparrow$ (.) hauptsächlich Schuelbehörde (.) und Jugendarbeit da in der Stadt ,Name der Stadt،. (1.5) Mir händ (.) wohnet sie in der Stadt?» Interviewerin: "Ja in 'Name des Stadtteilsı (.) ja (.) ja (.) ein bisschen am Rand ((lacht))." Herr Ifon: "Jaja ((lacht)). Zum Bispiel der Schuelchreis ,Name des Schulkreises، isch ja 'Name eines Stadtteils‘, 'Name eines anderen Stadtteilsı, 'Name eines weiteren Stadtteilsı (.) hätt mehr Schueler als zum Bispiel die feifgröschte Stadt in der Schwiz das Lausanne. (1.5) Einfach nur so für ( ) 28tuusig Schüeler in Stadt ,Name der Stadtı wo ind Volksschuel gönt (.) plus Tausende von Berufsschuel (.) Tausende von Kantonsschüeler 
also Abitur (.) wo Abitur machet plus no (.) Tausende vo Privatschüeler (.) und eigentlich mir händ i all dene Gfäss ine hän han ich Lüt wo v Verbindigsbeamte sind (.) zue de Schuelleitige (1.0) für niederschwellige Beratigen und so witer. (2.0) Also das isch sicher au ebbes sehr stark vernetzt eusre Mitarbeiter (.) mit dene zueteilte (.) Schuelkreise (.) Kantonsschule (.) Berufsschuele und so witer (.) das ist eigentlich so der (.) Haupt (1.0) und Usbildigzentrum für (.) Witerbildigszentrum für polizeiintern natürlich das isch klar." Interviewerin: "Ok (.) hmhm (.) und dann und Sie selber sind auch in all diesen ähm Bereichen tätig $\uparrow$ also sind sie auch (.) ähm auf der Straße?"

Herr Ifon: "Ich bin eher ( ) nei ( ) nicht mehr so viel nei (.) also nümmer so viel früher scho jetzt nümmer so viel also wirklich eher (.) eher als Leiter an sich sehr viel äh administrativs (.) und ähm strategisches und so witer also ich bin an und für sich nümmer so uf der Gass. (.) Aber ich weiß aber ich weiß was auf der Gass passiert wil (.) bi eus Stadtpoliezei ,Name der Stadt ‘ wenn sie $n$ Rapport schriebet $x$ welche Polizischt (.) gegen Jugendliche (.) der Jugendliche wird beschuldiget ebbes beganbe $z$ ha (.) Ladediebstahl (.) hätt kifft (.) er hätt (.) ebbes ebbes gstohle (.) hätt ibroche (.) die Rapört gönt alle da über minin Tisch (1.0) das sind dri a halb bis vier Tusig Rapört im Jahr (.) und dur das han ich natürlich scho a gwüsse Ahnig vo (.) äh (.) was lauft uf der Gass und so witer bloss natürlich vo der Patroullieberichte won ich weiß (.) ähm (.) mi steht ( ) det ha ma neue Schwerpunkt von Jugendliche wo sie treffet und so witer (.) was ma natürlich immer wieder feschtstellt isch das natürlich Jugendliche auch sich freirüm suechet $\uparrow$ (.) und mir ältere also- (1.0) Bevölkerig natürlich scho saget das isch schon guet und recht aber- (.) doch ned grad vo euserm Garte (.) äh hänge und und Alkohol trinke und Lärm mache Littering und so das chönnt ihr am andern Ort mache und nöd bei eus also die Jugendliche sind sind sie werden immer wieder verdrängt etcetera. (.) Und dann werdet mir wieder ( ) bizzle go (.) go schlichte und so witer (.) aber reine Präventionsabteilig wie mir au eine händ bei der Stadtpolizei ,Name der Stadtı das isch wirklich Prävention vorbeugend das machet die (.) mir kommen eigentlich denn (.) wenns ebbe schon passiert ischt. ( ) vo der Straftat kann aber sehr sehr präventiv wirke auf $n$ Jugendliche (.) wenn er jetzt plötzlich merkt ups (.) jetzt bin ich z wit gange. (1.5) A nacht (.) i der Zelle (.) und ( ) Besinnig (1.5) und ja." Interviewerin: "Ja (.) das glaub ich auch." Herr Ifon: "Nünzig Prozent vo de Jugendliche tun ja (.) eigentlich einmal händ die im Läbe z tue mit de Polizei und Jugendanwaltschaft. (.) Nünzig prozent (.) füf prozent (2.0) s bizzli mehr (.) und füf Prozent das sind dann die wo wo dann d Sorge bereitet (.) wo sehr uffällig sind (1.5) wo ständig delinqiere und so witer." (Zeilen 1-54)

Das Interview mit Herrn Ifon hat einen Vorlauf, der nicht aufgenommen worden ist, da er zwischen Tür und Angel stattgefunden hat und deshalb fehlt eine Einstiegsfrage. Ihm ist zunächst wichtig, seine Biografie zu schildern, wobei er primär berufliche Entwicklungen, sekundär aber auch private Informationen relevant setzt. Dass er seine Kinderlosigkeit auf seine beruflichen Erfahrungen zurückführt, lässt sich zwischen Ironie und Zynismus einordnen, kann in 
diesem Zusammenhang aber auch darauf hinweisen, dass er seine »private« Person stark mit seiner beruflichen Identität verknüpft. Obwohl er nicht direkt mit der Adressierung als Experte für Jugendgewaltprävention konfrontiert ist, distanziert er sich in gewisser Weise davon, indem er angibt, dass Prävention nicht im Fokus der Jugendpolizeiarbeit steht, sondern Ermittlung. Als stellvertretender Leiter des Jugenddienstes einer Stadtpolizei expliziert und impliziert er schließlich, was Prävention nicht ist, nämlich Ermittlung. Prävention als »vorbeugende« Maßnahme sei Sache der Abteilung »Prävention«. Somit rekonstruiert er seine Expertise in der Abgrenzung zu Jugendgewaltprävention. Der Jugenddienst der Polizei grenze sich von anderen Abteilungen ab, indem er »alles macht«, was mit »Beschuldigten unter $18 \ll$ zu tun habe. Schließlich distanziert er sich wiederum vom Ermittlungsdienst, insofern er angibt, nicht mehr »auf der Gasse« zu arbeiten, sondern primär administrativ tätig zu sein. Jedoch sei er sehr gut informiert, da alle relevanten Informationen »über seinen Tisch gehen«. Insgesamt vermittelt Herr Ifon das Bild, Jugendgewaltprävention sei nicht Gegenstand der Polizei, wobei andererseits Strafe auch präventiv wirken könne. Auffällig ist die Bedeutung von Zahlen und Mengen, die mit seiner Tätigkeit einhergehen. In der Stadt gebe es »Tausende« Schülerinnen und Schüler, »tausende « Berichte im Jahr gingen über seinen Schreibtisch. Jugendliche kommen Herrn Ifon in Form von Zahlen in den Blick. Darüber hinaus differenziert er zwischen Jugendlichen und »den Älteren«, zu denen er sich auch selbst zählt. Damit vermischt er seine Sprecherpositionen als Leiter des Jugenddienstes der Polizei und als Vertreter einer Generation. Aus der Perspektive der Älteren bewertet er das Handeln Jugendlicher, die ja nicht vor »ihrem Garten hängen und Alkohol trinken müssen, Lärm machen und Littering«. Aufgabe des Jugenddienstes, also auch seine Aufgabe, sei es schließlich, wieder zu schlichten. Herr Ifon vermischt also nicht nur private und berufliche Aspekte seiner Biografie, sondern auch seine Positionen als Vertreter einer »älteren Generation« und als Leiter des Jugenddienstes. Die Differenz zwischen den Generationen impliziert eine »Normalisierung« von Jugendgewalt in dem Sinne, dass sich Jugendliche »natürlich Freiräume suchen«. Davon grenzt Herr Ifon die kleine Gruppe von fünf Prozent ab, die »Sorge bereiten«, die »auffällig sind oder die ständig delinquieren und so weiter«. Die Normalisierung von Jugendgewalt geht hier also gleichzeitig mit einer Skandalisierung einher, die über gewaltige Zahlen sowie über die kleine Gruppe der »Auffälligen« konstruiert wird.

\section{Herr Schäfer, Jugendrichter}

Interviewerin: "Vielleicht gleich zu Beginn ähm (.) mit ist nicht so ganz klar was die Jugendanwaltschaft für eine Funktion hat. (.) Vielleicht können Sie das einfach mal erklären ((lacht))."

Herr Schäfer: "Ja das isch schnell erklärt. (.) Soll ich Hochdütsch rede?" 
Interviewerin: "Wie Sie möchten also ich verstehe Beides."

Herr Schäfer: "Sie verstöns gued ja dann (.) blieb ich so ja. (.) Also mir händ Jugendawaltschafte sind einerseits Jugendstrafverfolgigbehörde $\uparrow$ (.) also mir machet Strafuntersuechige führ ma dure. (.) lii über zünzg Prozent der Fälle mach ma auchs Urteil (.) und mir vollziehend nachher auch d Urteil oder das heißt vor allem bei dene Jugendliche wo (.) a Maßnahme überbechömmet da ambulante wo mer in eigener Kompetenz chönnt machen oder a stationäre wo s Jugendgericht letschtlich dann muss aordne (.) machet mir au de ganze Vollzug (.) bis eigentlich die Maßnahm wieder ufghobe wird ja und Nachbe Nachbetreuig alles au (.) drum schaffet uf d Jugendawaltschafte a ned nur Jurischte wie ich sondern au Sozialarbeiterinne. (.) Mir schaffet also eigentlich sehr näch zamme."

Interviewerin: "Was sind die verschiedenen Aufgaben von also Sozialarbeitern was machen die und was machen Sie als Jurist?"

Herr Schäfer: "Also mir die erschte Ufgabe isch äh bei dene Jugendliche bei dene mir s Gfühl händ mir münd gnauer ranluege dass sie d Abklärig mache zur Person also wo lieget d Problem was (.) und en Vorschlag machet was machet ma da (.) was machet ma da sinnvoller wies. (.) Sie begleitet die Jugendliche dann au während dem zum Bispiel immen ne Heim sind oder bi ambulante Behandlige bi ambulante Betreuige führet sie sie selber dure oder dann hän mer Ußerstehende es gibt a so Firmene wo wo sozialpädagogische Familiebegleitige machet also sie sind dann eigentlich so chli en Advicasemanager wo dann die Lüt isetzet."

Interviewerin: "Und (.) vertreten Sie dann auch Jugendliche (.) also vor dem Gericht oder."

Herr Schäfer: "Nei (.) oder vo mir sind Ansich so vo de vom suschtige Strafrecht her sin mir de Ankläger (.) nur hän mir ebe so ne Mischfunktion es isch einersits wege mir mache die Achlag auf Gricht (.) aber äh glichzitig (.) hän mir eigentlich so vom Jugendlichen im Auge $z$ bhalte und im Gspräch z bhandle es is so a chli Mischig (.) vor allem in Dütschland immer a chli zur Verwirrig Alass git ((lacht))."

Interviewerin: "Ja ok. (.) Ich kannte das auch nicht aber ich hab mich in Deutschland auch nicht (.) damit beschäftigt als für mich ist des (.) generell neu."

Herr Schäfer: "Ja mei das isch so speziell dass es einersits Juristerei isch andrersits Sozialarbeit und dann au no Pädagogik."

Interviewerin: "Hmhm (.) ok (.) interessant."

Herr Schäfer: "Und es Ziel vom Jugendstrafrecht isch ja eigentlich eben des soll es pädagogisch orientierts Strafrecht si und eben nöd es Verschuldensstrafrecht wie mirs bi den Erwachsene händ."

Interviewerin: "Hmhm (.) ok (.) und können Sie mir vielleicht schildern wie so ihr typischer Arbeitsalltag aussieht?"

Herr Schäfer: "Ja ich denks (.) s typische is eigentlich dass es chei typische git ((lacht)). Also isch ja spannend ma weiß eigentlich nie was was wieder los isch also ebbe ich tun (.) Ivernahme vorbereite hab Gspröch mit Eltere hab Gspröch mit Jugendliche (.) ich muss denn de Untersuechigshaft aordnen also man bekommts Telefon über dan gaht 
ma mer in Polizeikaserne für Ivernahm mache und entscheide obs Haft gäb oder nöd. Mir bsuechet Heim was es gibt ja regelmäßig nöd nur Heim also überhaupt also bei andern stationäre Unterbringige ob öbber zum Bispiel in einzelbetreuts Wohne (.) macht (.) da gits eifach regelmäßig sogenannte Standortssitzige (.) wo mer immer wieder s Gliche macht man hät sich gwüsse Ziel gsetzt ma luegt wie wit si mer cho was laft gued was laft nöd gued (.) wo mü mer ebbes no verändere. (.) Ja also es isch äh (.) a rächte Mischig (.) und danebe muss ich natürlich noch den Ladde da führe ((lacht))."

Interviewerin: "Ja (.) und also inwieweit wie sieht denn Ihre Arbeit mit den Jugendlichen aus?"

B: "Ja dass isch natürlich individuell sehr unterschiedlich also ich ich schaffe mit Jugendliche sehr sehr direkt und unkompliziert also genau wie ich jetzt mit Inne dasitze hock ich a mit dem Jugendliche da das isch äh ja ich probier eigentlich ich ich bin natürlich $n$ Verfechter also und a überzügt davo (.) dass mer sinnvolle Arbeit nur chönnet mache über d Beziehig dass mer münd a vernünftige Beziehig annebringe (.) dass er mir irgenwann glaub dass ichs eigentlich schon gued meine villiecht falsch mache aber äh er soll mir wenigschtens glaube dass ichs gued meine ((lacht)). Ich han so drü sätz oder $\uparrow$ wo für mich so $d$ Basis sind wo ich probier z erreiche mit den Jugendlichen dass er merkt ich mag inn (.) er isch mir wichtig und ich trau inm ebbes zue. (.) Des is so chli mini (.) Philosophie won ich wolt probiere annezbringe und das isch i der Regel a nöd sehr schwierig."

Interviewerin: "Ok (.) und was sind des für Jugendliche (.) was haben die (.) Probleme oder für ein Problem die hier herkommen?"

B: "Ja musch da natürlich sehn der Gröschte Teil isch eigentlich unproblematisch die händ dann Grenzüberschritig gmacht wie Sie (.) wie ma ja us Dütsche Studie weiß vor allem für männliche Jugendliche sehr normal isch (.) dass mer das macht. (.) Und es git $\mathrm{n}$ chlini Prozentsatz dös sind villiecht so acht Prozent wo man merkt da da sind wirklich Problem umme (.) und die chönnt dann natürlich so die drü wichtigschte Bereich sind ja Familie (.) Schuel oder Bruef (.) und Freizitverhalte (.) und da hammer halt i ein in der Stadt ,Name der Stadt، hän mer natürli grad was so familiäre Hintergründ agaht (.) kombiniert mit Migrationshintergrund zum Bispiel mit Kriegserfahrige (.) im Balkan i de letschte Jahre vor allem (.) hä mer zum Teil sehr desolate Verhältnisse bei dene da sind wenig dös muess ma immer wieder sage aber es sind eifach die wo uns natürlich dann extrem lang (.) beschäftiget. (.) Also mir chönnt ja (.) s Jugendstrafrecht fangt bei zähne a (.) Delikt bis achtzähne fallt bei eus ind Kompetenz a Maßnahm chann sogar bis zwoazwanzge gah also mir händ zum Teil schaff ich mit Jugendliche also mit der längschte Fall isch ebbe elf Jahr (.) wo mer mit dem sich beschäftigt." (Zeilen 1-65).

Auf die Bitte der Interviewerin, die Funktion des Jugendgerichts zu erklären, schildert Herr Schäfer seinen Tätigkeitsbereich zunächst unter der Verwendung von Fachtermini, woraus schließbar ist, dass er einen gemeinsamen Wissenshintergrund voraussetzt. Möglicherweise geht Herr Schäfer davon aus, dass die Interviewerin sich gut informiert hat bezüglich der Funktion 
der Jugendanwaltschaft, was sie allerdings im weiteren Verlauf des Gesprächs nicht anzeigt. Die Jugendanwaltschaft habe einen sehr umfassenden und umfangreichen Aufgabenbereich, der bei der Strafverfolgung beginne und bei der Nachbetreuung aufhöre. In diesem Prozess sei es relevant, dass Juristen und Sozialarbeiterinnen zusammenarbeiten, wobei er die Relevanz von Jus, Sozialarbeit und Pädagogik für die Jugendanwaltschaft betont. Auf die Frage, inwiefern er mit Jugendlichen arbeite, setzt er das Gespräch mit der Interviewerin implizit gleich mit den Gesprächen, die er mit den Jugendlichen hat und die er als direkt und unkompliziert beschreibt. Seine Strategie oder auch »Philosophie« bestehe darin, eine »vernünftige Beziehung herzustellen« und den Jugendlichen Glauben zu machen, dass er es gut meine mit ihnen. Der Gros dieser Gespräche besteht demnach weniger in der Herstellung von Empathie als in der Darstellung von Glaubhaftigkeit und daran anschließend im Evozieren einer bestimmten Erwartungs- oder Anspruchshaltung der Jugendlichen. Den Anspruch, glaubhaft zu sein, überträgt Herr Schäfer implizit auf die Interviewsituation: Der Verweis darauf, dass seine Philosophie aufgehe, kann also als Anspruch interpretiert werden, der Interviewerin seinen Erfolg glaubhaft zu machen. Dies würde darauf schließen lassen, dass der Befragte den Inhalt des Gesprächs als heikel ansieht und er Kritik erwartet, die Interviewerin also als potenzielle Kritikerin wahrnimmt. Dieses Interaktionsmuster lässt sich bereits in anderen Interviews herauslesen, was die Annahme bestärkt, dass es sich beim Feld der Jugendgewaltprävention um ein vulnerables Feld handelt und um ein Feld, in dem es besonders wichtig ist, Erfolge sichtbar zu machen. Auf die Nachfrage der Interviewerin nach den Jugendlichen, mit denen Herr Schäfer arbeitet, differenziert er zwischen zwei Gruppen. Die meisten der Jugendlichen hätten »Probleme«, die Herr Schäfer in Bezug auf wissenschaftliche Studien als »Grenzüberschreitungen, [...] die vor allem für männliche Jugendliche sehr normal ist«, bezeichnet. Damit naturalisiert und normalisiert er die Jugendphase insbesondere über die Differenzierung nach der Kategorie Geschlecht. Demgegenüber stehen Grenzüberschreitungen, die »pathologisch« seien - wo man merke, dass da wirklich Probleme vorliegen würden. Diese »pathologischen« Probleme schreibt Herr Schäfer den jeweiligen »desolaten Verhältnissen« in Bezug auf »Familie«, »Schule«, »Beruf«, »Migrationshintergrund « sowie »Kriegserfahrungen im Balkan« zu. Damit differenziert Herr Schäfer implizit zwischen »normaler« Jugendgewalt, die durch »biologische« Prozesse determiniert ist, und »pathologischer« Gewalt, die über gesellschaftliche Defizite wie »schlechte Erziehung, Krieg und mangelnde Integration zu erklären ist. Diese zweite Gruppe von Jugendlichen seien die eigentlich relevanten Klienten des Jugendgerichts. 


\section{Zusammenfassender Fallvergleich: "(Un-)Doing Expertise" - Zur Relativierung von Adressierungsweisen und Interaktionsdynamik}

Obwohl die Befragten in der Interaktionssituation der Interviews nicht immer, aber meistens direkt als Expertinnen bzw. Experten für Jugendgewaltprävention adressiert werden, im Sinne einer Aufforderung, »adäquat« über das Kernproblem dieser Thematik zu sprechen, weisen die Interpretationen auf Tendenzen der Relativierung einer zugeschriebenen Position hin: Auf Distanzierungen gegenüber der durch die Interviewerin gemachten Zuschreibungen und Adressierungsweisen folgen Reformulierungen der eigenen Expertise. Relativierungstendenzen lassen sich möglicherweise auch auf die Adressierung zurückführen, die über das Anschreiben vorgenommen worden ist. Dafür sprechen auch Beobachtungen, dass sich einige der Befragten sogar schriftlich auf das Interview vorbereitet haben (sehr deutlich zeigt sich dies am Interview mit Herrn Tanner, worauf in folgenden Interpretationen noch zurückgekommen wird). Diese Adressierungsweisen enthalten bestimmte Erwartungen, von denen sich die Befragten zwar distanzieren können, dabei jedoch gleichzeitig in den Zwang geraten, die jeweils eigenen Expertisen zu explizieren. Die Explikation erfolgt in der Distanzierung von der Figur, die ihnen zugeschrieben wird. Demnach rekonstruieren die Befragten ihre eigene Expertise an den Grenzen der ihnen zugeschriebenen Figur einer Expertin bzw. eines Experten. Diese Interpretation lässt darauf schließen, dass es sich bei der Relativierung des Status einer Expertin bzw. eines Experten nicht um eine dem Feld der Jugendgewaltprävention eigentümliche Strategie handelt, sondern die Relativierung dem Erhebungsinstrument geschuldet ist. Allerdings weisen die Distanzierungsweisen auch darauf hin, dass die Interviewerin als potenzielle Kritikerin oder Evaluatorin wahrgenommen wird: Die Befragten verweisen auf die Notwendigkeit, Erfolge ihrer Präventionsarbeit sichtbar zu machen, und legen darauf Wert, dies auch der Interviewerin glaubhaft darzustellen. Diese Beobachtungen könnten der Feldspezifik geschuldet sein, insofern diese durch ein kriminalpolitisches Programm strukturiert ist, das zum Ziel hat, über Evaluationen eine Standardisierung von Maßnahmen hinsichtlich der Jugendgewaltprävention einzuführen und zu etablieren, und im Zusammenhang mit verschiedenen Diskursen bzw. Diskursfragmenten steht. In dieser Hinsicht kann die Beobachtung der Relativierung des Status einer Expertin bzw. eines Experten als Distanzierung von diskursiv vermittelten und vermittelbaren Inhalten durch die Forscherin angesehen werden. Eine (Re-)Konstruktion von Deutungsmustern der Jugendgewalt und Jugendgewaltprävention erfolgt primär anhand von Interviewsequenzen, in welchen die Befragten ihre je eigenen Relevanzen entwickeln. Bevor diese Beobachtungen methodisch und theoretisch gedeutet und diskutiert werden, wird zunächst zusammengefasst, auf welche Weise die Befragten den ihnen zugeschriebenen Status einer Exper- 
tin bzw. eines Experten relativieren. Die der hier einführend formulierenden Interpretation zugrunde liegenden Sequenzen haben zwar nicht primär erzählerischen Charakter, können jedoch im Sinne der dokumentarischen Methode insofern als Fokussierungsmetaphern, die im Folgenden reflektierend interpretiert werden, bezeichnet werden, als die Befragten selbst diejenigen sind, die auf widerständige Weise eine Thematik fokussieren und damit das, was sie selbst als problematisierungswürdig erachten, einfordern (vgl. Bohnsack 2001: 233f.). Somit geben die Interpretationen dieser Sequenzen nicht nur Aufschluss darüber, welche Gegenstände in den jeweiligen Kontexten problematisiert werden, sondern auch, welche Expertisen und damit verbundenen Problematisierungs- und Deutungsweisen in Bezug auf Jugendgewalt und Jugendgewaltprävention vorliegen. Im Folgenden werden die interpretierten Einstiegspassagen anhand methodischer und inhaltlicher Aspekte reflektiert. Es wird zusammenfassend dargestellt, wie die Befragten sich den Zuschreibungen der Forscherin entziehen und wie sie diese wiederum selbst adressieren. Folglich konzentrieren sich die Interpretationen auf Interaktionsdynamiken der jeweiligen Interviews. Dabei werden außerdem Aspekte der jeweiligen Orientierungsrahmen - die Positionierungen in den Kontext eines gesellschaftspolitischen Problems, Problematisierungsweisen im je spezifischen Arbeitsfeld sowie die Darstellung der eigenen Expertise - thematisiert, die auf Konstruktionsweisen von Gewalt und Prävention hinweisen. Dies ermöglicht eine Einschätzung des Vergleichspotenzials bezüglich der Deutungen von Gewalt und Prävention, die in den Kapiteln 4.1.2 und 4.1.3 herausgearbeitet werden. Eine zusammenfassende methodische Diskussion befasst sich weiter mit der Frage, inwiefern die beobachtete Relativierung des zugeschriebenen Status einer Expertin bzw. eines Experten der Erhebungsmethode oder dem Feld geschuldet ist. Theoretisch nähert sich die hier vorliegende Arbeit schließlich einem ihrer zentralen Themen an: dem des Subjekts.

\section{Herr Unterwasser, Fachbereichsleiter}

Herr Unterwasser distanziert sich nicht nur von der Arbeit mit Jugendlichen, sondern er problematisiert diese sogar. Der Arbeit mit Jugendlichen, die diese als mühsam erleben, stellt er die Arbeit mit Erwachsenen gegenüber, u.a. mit seinen »Fachleuten«, an die er die Arbeit mit Jugendlichen delegiert. Diese »Strategie« stellt er jedoch als legitimationsbedürftig dar, indem er auf Erfolge im Kontext eines äußeren Erfolgsdrucks verweist. »Männliche« Erwachsene sind für ihn der Maßstab, an dem er sich gewissermaßen abarbeitet und worin er seine Expertise sieht. Diese Arbeit erfordert im Wesentlichen das Inszenieren von Erfolgen unter einem äußeren Erfolgsdruck, was er als problematisch ansieht. Die Erfahrung, dass Jugendliche die Arbeit mit ihm als mühsam erleben, wirkt auf ihn zurück: Indem er diese Arbeit delegiert und seine Expertise in der wissenschaftlichen Reflexion sucht, entzieht er sich dieser »Mühsam- 
keit«. Möglicherweise erlaubt ihm der so gerahmte Arbeitskontext kein erfahrungsbasiertes Wissen über Jugendgewalt. Wissen über Jugendgewalt ruft er stattdessen über den Rekurs auf wissenschaftliche Theorien ab. In der Distanzierung von der Arbeit mit Jugendlichen zugunsten der Arbeit mit Erwachsenen und wissenschaftlichen Erkenntnissen relativiert Herr Unterwasser seine Expertise hinsichtlich Jugendgewaltprävention. Er arbeitet insbesondere auch seine eigene Biografie, die Biografie eines nicht gewalttätigen erwachsenen Mannes, retrospektiv an Theorien ab. Dies ermöglicht ihm, Gewalt als etwas zu erfahren, das ihn selbst in seiner persönlichen und professionellen Entwicklung geprägt hat, und seine Theorien darüber damit für sich selbst $\mathrm{zu}$ bestätigen. In dieser Hinsicht rekonstruiert er Gewalt als wissenschaftlich erzeugtes Wissen, das ihn in seiner persönlichen und professionellen Entwicklung geprägt hat - ein Hinweis darauf, dass er diese beiden Rollen miteinander verwebt. Das theoretisch angereicherte Deutungsmuster von Gewalt beschreibt, wie »junge männliche Menschen« geprägt werden, und weist Männern primär einen Opferstatus, gleichzeitig jedoch auch einen Täterstatus zu, der irreversibel ist. Gewalt determiniert Biografien heranwachsender Männer: Täter kann nur sein, wer Opfer ist. Gewalterfahrungen verfestigen sich, bis sie zum Bestandteil der Persönlichkeit werden. Erwachsene Männer sind laut Herrn Unterwasser somit gesellschaftlicher Maßstab, mit dem er sich identifiziert und an dem er sich abarbeitet. Er problematisiert Gewalt in ihren (biografischen) Ursachen und adressiert heranwachsende Männer als besonders vulnerable Gruppe. Herr Unterwasser unterstellt der Interviewerin, die Theorien, die für seinen Arbeitskontext relevant seien, bereits zu kennen, kontextualisiert dieses Wissen aber: Er expliziert, wie und warum dieses Wissen für seinen Arbeitskontext relevant ist. Er stellt sich somit als Nutzer dieses Wissens in seinem Handlungskontext dar, in dem es relevant ist, Biografien zu rekonstruieren. Relevant sei, zu erklären, wie Täter zu Tätern geworden seien. Auch das Abarbeiten der Theorien an der eigenen Biografie bzw. den Biografien anderer Erwachsener erachtet Herr Unterwasser als relevant, was auf eine geringe persönliche Distanz zur eigenen »professionellen« Rolle schließen lässt. Die Betonung »wirklicher« Erfolge seiner Arbeit könnte auch darauf hinweisen, dass er die Interviewerin nicht nur als Expertin einer anderen Wissenskultur ansieht, sondern auch als Autorität oder potenzielle Kritikerin, vor der er sich zu legitimieren sucht. Möglicherweise muss er sich vor seinen Auftraggebern rechtfertigen, hat bereits Erfahrungen mit Kritik und/oder Evaluation gemacht hat und projiziert diese Erfahrung auf die Interviewsituation. Dies könnte auch darauf hinweisen, dass es sich bei Jugendgewaltprävention um einen vulnerablen Deutungs- und Handlungskontext handelt, in dem Evaluationen einen hohen Stellenwert einnehmen, insofern als sie wirkmächtig in spezifische Handlungs- und Deutungskontexte einfließen. In Bezug auf eine kriminalpolitische Programmatik ist herauszustellen, dass Herr Unterwasser 
ein gewisses Legitimations- und Anerkennungsbedürfnis seiner Arbeit nach außen formuliert, das sich auch in der Interviewsituation spiegelt, insofern als er der Forscherin seinen Erfolg darstellt. Dies erfolgt jedoch nicht auf unkritische Weise. Es hat den Anschein, als müsse er seine Expertise nach außen hin rechtfertigen, indem er Erfolge sichtbar macht, z.B. durch mediale Inszenierung, zu der er eigentlich ein kritisches Verhältnis hat. Im Bestreben, Erfolge der Jugendgewaltprävention v.a. im Sinne ihrer Wirksamkeit sichtbar zu machen, schließt Herr Unterwasser an programmatische Inhalte an, die sog. »evidenzbasierte« Projekte idealisieren und unterstützen. Diese Orientierung erfolgt nicht unkritisch, zumal er seinen Expertenstatus hinsichtlich seiner Expertise, nicht mit Jugendlichen, sondern mit Erwachsenen zu arbeiten, relativiert. In dieser Hinsicht könnte die Relativierung des Expertenstatus als Distanzierung zu kriminalpolitischer Programmatik verstanden werden. Andererseits fällt auf, dass das Wissen, das er für Jugendgewaltprävention relevant setzt und über das er sich auch »professionalisiert«, kriminologisch dem relevant gesetzten Wissen über Ursachen von Jugendgewalt entspricht. Herr Unterwassers Beschreibungen enthalten also gewisse Ambivalenzen. Er unterscheidet implizit zwischen »normaler « und »pathologischer« Jugendgewalt, indem er Gewalt nur in Bezug auf »männliche Täterkarrieren«, die irreversibel sind, problematisiert. Er versteht Gewalt als Ergebnis einer Erduldungsbiografie, die jemanden zum Opfer macht. Der Täter sei Opfer gesellschaftlicher Prozesse, die sich auf individuelle Biografien auswirkten. Diese zu verstehen, scheint Kern seiner Expertise zu sein, wozu er auf wissenschaftliches Wissen rekurriert. Er bewertet Täter unter Einbezug wissenschaftlich anerkannten Wissens, das als »kriminalprogrammatisch« zu werten ist. Dieses Wissen lässt sich jedoch nicht ohne Weiteres auf die Arbeit mit Jugendlichen »anwenden«, da diese noch keine »Täterkarriere« aufweisen können, womit Jugendgewalt implizit normalisiert wird. In Bezug auf Jugendgewaltprävention sieht Herr Unterwasser das Verstehen von Täterkarrieren aus wissenschaftlicher Perspektive als besonders relevant an, was, wie bereits erwähnt, »kriminalprogrammatisch « kompatibel ist. Dies ist jedoch legitimationsbedürftig, was sich an dem Bestreben zeigt, Erfolge auszuweisen. Diese Relevanzsetzung ist plausibel vor dem Hintergrund einer Normalisierung von Jugendgewalt. Jugendgewaltprävention deutet Herr Unterwasser als Verstehensprozess und nicht als Arbeit mit Jugendlichen.

\section{Frau Schock, Familientherapeutin und Jugendarbeiterin}

Frau Schock distanziert sich zunächst von der Adressierung als Expertin für Jugendgewalt, indem sie ihre Expertise als Familientherapeutin expliziert und ihrer Erfahrung als Jugendarbeiterin in der aufsuchenden Jugendarbeit, auf die sie später noch zu sprechen kommt, vorzieht. Ihre Arbeit bestehe primär in der Arbeit mit Familien und sekundär mit Kindern. Ihren Schilderungen 
nach ist ihre Rolle als Familientherapeutin von einer Situation der Ambivalenz geprägt. Sie nimmt gleichzeitig eine Perspektive auf Familie und innerhalb von Familie ein. Frau Schock problematisiert nicht Jugendgewalt an sich, sondern die diesbezügliche Erwartungshaltung ihrer Auftraggeberinnen bzw. Auftraggeber aus Erziehungs- und Bildungseinrichtungen. Sie sei beauftragt, die Probleme zu lösen, mit denen diese konfrontiert seien. In dieser Hinsicht problematisiert sie, ähnlich wie Herr Unterwasser, ihren Arbeitskontext, der von außen gerahmt ist und der ihr kein Erfahrungswissen bezüglich Jugendgewalt zur Verfügung stellt bzw. dieses nicht benötigt. Ihre Arbeit als Familientherapeutin legitimiert sich über die Annahme der Auftraggeberinnen bzw. Auftraggeber darüber, Jugendgewalt sei in seinem Ursprung in Familien situiert. Konsequenterweise sieht sich Frau Schock nicht mit dem Problem von Jugendgewalt, sondern mit dem Ursprung von Jugendgewalt konfrontiert. Insofern geht sie mit den Problematisierungsweisen ihrer Auftraggeberinnen bzw. Auftraggeber konform. Den Ursprung von Jugendgewalt erfasst sie über einen Rekurs auf psychologische Theorien, die den »Ursprung« von Jugendgewalt relevant setzen. Frau Schock stellt ihre Expertise als Psychologin der Expertise der Interviewerin als Soziologin gegenüber. Dabei betont sie die Bedeutung von Bindungen und Beziehungen, was auch von ihren Auftraggeberinnen bzw. Auftraggebern als relevanter Ursprung von Gewalt problematisiert wird. In dieser Hinsicht lässt sich ihre Expertise als Familientherapeutin mit den Annahmen der Auftraggeberinnen bzw. Auftraggeber bezüglich des Ursprungs von Gewalt vereinbaren, jedoch sieht sich Frau Schock nicht in der Lage, Probleme in ihrer Arbeit als Familientherapeutin einfach »mit dem magischen Stab« zu »lösen«. Sie expliziert hier einen Konflikt zwischen den Erwartungen der Auftraggeberinnen bzw. Auftraggeber und den Möglichkeiten ihrer Arbeit. Mit dem Phänomen Gewalt sieht Frau Schock sich selbst nicht konfrontiert, sondern ihre Auftraggeberinnen bzw. Auftraggeber. Frau Schock hingegen ist auf der Suche nach dem Ursprung von Gewalt, der jedoch nicht ohne Weiteres auffindbar und lösbar ist. Frau Schock problematisiert den hohen Einfluss der Bildungs- und Erziehungseinrichtungen auf Behörden und Familien und den damit einhergehenden Erfolgsdruck. Frau Schocks Arbeitskontext ist somit von hoher Ambivalenz geprägt. Einerseits positioniert sie sich kritisch zum Auftrag, andererseits sieht sie sich in ihrer Arbeit als Familientherapeutin legitimiert, indem sie genau an dem »Ort« ansetzen kann, an dem ihre Auftraggeberinnen bzw. Auftraggeber den Ursprung des Problems Jugendgewalt sehen, nämlich in der Familie. Somit problematisiert Frau Schock weniger ihre Tätigkeit selbst als vielmehr den damit einhergehenden Erfolgsdruck. Frau Schock geht konform mit der Annahme ihrer Auftraggeberinnen bzw. Auftraggeber, den Ursprung von Jugendgewalt in den Familien zu verorten. Somit setzt sie die Frage des Ursprungs relevant. Ihre Auftraggeber hingegen seien mit dem Phänomen selbst konfrontiert, das sie mit Frau Schocks 
Hilfe beseitigen wollten. Zu dieser Erwartung verhält sich Frau Schock kritisch. Die Ambivalenz gegenüber den Erwartungen der Auftraggeberinnen bzw. Auftraggeber spiegelt sich in ihrer gleichzeitigen Positionierung in der Familie und außerhalb der Familie wider. Indem Frau Schock den Ursprung des Problems relevant setzt, führt sie ihre Expertise als Familientherapeutin ein. Gegenstand ihrer Arbeit sei nicht Gewalt, beispielsweise in der Verkörperung eines »verhaltensauffälligen« Kindes, sondern die Familie. Frau Schock sieht sich mit der Verantwortung dafür konfrontiert, dass Probleme in den Institutionen der Bildung und Erziehung gar nicht erst in Erscheinung treten, Kinder also gar nicht erst »verhaltensauffällig« werden. Die Suche nach dem Ursprung des Problems erfordere den Einbezug wissenschaftlich etablierten Wissens. Frau Schock differenziert also zwischen Gewalt als Phänomen, mit dem sie selbst nicht konfrontiert ist, und dem Ursprung von Gewalt, den sie zu verstehen versucht, indem sie ihn aufsucht und dabei auf wissenschaftliche Theorien rekurriert. Gewalt ist aus ihrer Perspektive familialen Dynamiken unterworfen, die sie zu verstehen versucht. Frau Schocks Präventionsarbeit besteht im Verstehen sozialer Dynamiken der Entstehung von Gewalt in Bezug auf wissenschaftlich etabliertes Wissen. Dabei wirkt es zunächst problematisch, dass sie mit Gewalt selbst nicht konfrontiert ist. Sie verlässt sich auf die Problematisierungsweisen ihrer Auftraggeberinnen bzw. Auftraggeber. Von deren Erwartung, dass sie ihre Probleme mit dem »Zauberstab« lösen könne, distanziert sie sich, steht dieser also kritisch gegenüber. Sie sieht es also nicht als ihre Aufgabe an, Probleme zu lösen, sondern will sie verstehen. Diese Situation ist hoch ambivalent. Einerseits geht Frau Schock konform mit ihren Auftraggeberinnen bzw. Auftraggebern, was die Relevanz und den Ursprung von Gewalt betrifft, andererseits hat sie ein anderes Verständnis von Prävention im Sinne dessen, was sie bewirken kann. In gewisser Weise distanziert sie sich von dem Druck, den sie durch ihre Auftraggeber erfährt. Die Relevanzsetzung der Frage nach dem Ursprung von Gewalt plausibilisiert die Arbeit an dem Ort, der als Ursprung angesehen wird: in der Familie. Die soziale Dynamik in Familien, die die Entstehung von Gewalt begünstigt, gilt es zu verstehen, was Frau Schock als ihre Expertise ansieht.

\section{Herr Gold, Leiter einer privaten Organisation}

Herr Gold weist die ihm zugeschriebene Expertise bezüglich der Problematisierung von Jugendgewalt insofern zurück, als er nicht auf der Ebene seiner Arbeit als Leiter einer Organisation, sondern auf einer allgemeinen gesellschaftskritischen Ebene agiert. In dieser Hinsicht gibt er sich primär als Gesellschafts- und Medienkritiker aus, der die dauernde mediale und gesellschaftliche Problematisierung von Jugendgewalt problematisiert. Er sieht Jugendgewalt als historische Konstante an, was eine Art von Normalisierung impliziert. Diese Normalisierung von Jugendgewalt bricht aus seiner Perspek- 
tive mit deren Skandalisierung: Er setzt der medialen und gesellschaftlichen Skandalisierung von Jugendgewalt deren Normalisierung entgegen. Herr Gold bricht auf die Frage der Interviewerin hin in schallendes Gelächter aus und bezeichnet die Einstiegsfrage als »heiße Frage«, was in diesem Kontext ironisch verstanden werden kann. Daraufhin gibt er seine gesellschaftskritische Meinung wieder. Die Ironisierung der Einstiegsfrage und das Sprechen aus einer gesellschaftskritischen Perspektive, in der er seine fachspezifische Erfahrung ausblendet, weist darauf hin, dass er die Interviewerin als Laie ansieht. Dieser Eindruck wird durch die Beobachtung bestärkt, dass Herr Gold seiner eigenen Meinung eine Deutungshoheit zuerkennt. Er problematisiert den gesellschaftlichen Diskurs über Jugendgewalt dahingehend, dass die Gesellschaft ihre Probleme auf Jugendliche projiziert. Diese Haltung verunmöglicht, Jugendgewalt auf ein konkretes Problem hin zu reduzieren, wozu er sich durch die Einstiegsfrage womöglich aufgefordert sieht. Er distanziert sich in dieser Deutung von der Erwartung der Interviewerin, das Problem auf einen Punkt zu bringen. Vor dem Hintergrund dieser Distanzierung führt er seine Expertise als »Gesellschaftskritiker« ein, der er eine hohe Relevanz einräumt. Jugendgewalt könne zwar hinsichtlich seiner Ursachen diskutiert, nicht aber per se problematisiert werden.

\section{Herr Vonderstatt, Leiter einer städtischen Jugendarbeit}

Herr Vonderstatts »Haltung« bzw. »Meinung« ist stark von einer entwicklungspsychologischen Perspektive auf Jugendliche geprägt. Diese kontextualisiert er nicht im Sinne einer fachbereichsspezifischen Erfahrung, was als Distanzierung von einer Adressierung als Experte für Jugendgewaltprävention gewertet werden kann. Diese Distanzierung verstärkt er, indem er die Einstiegsfrage ironisiert, die er als »Gretchenfrage « bezeichnet. Ähnlich wie Herr Gold bezieht er seine »Meinung « bzw. »Haltung« nicht auf seinen Fachbereich. Aus dieser Perspektive heraus differenziert er implizit zwischen »normalen« Formen von Jugendgewalt in Bezug auf die »Entwicklungsphase« und einer »problematischen« Form von Jugendgewalt. Über diese Differenzierung entscheide die Gesellschaft. Damit macht er eine Differenz zwischen »der Gesellschaft« und »den Jugendlichen« bzw. »jugendlichem Handeln« implizit. Gesellschaft habe die Legitimation, jugendliches Handeln zu bewerten. Andererseits könne jugendliches Gewalthandeln auch ein individueller »Hilfeschrei« sein, womit Herr Vonderstatt Jugendliche als »hilfsbedürftige« soziale Gruppe adressiert.

\section{Herr Tanner, Leiter der offenen Jugendarbeit einer Stadt}

Herr Tanner distanziert sich von der Adressierung als Experte für Jugendgewaltprävention, insofern er biografisch von Jugendarbeit Abstand nimmt. Die Arbeit mit Jugendlichen im Sinne einer Beziehungsarbeit nimmt in seiner Be- 
rufsbiografie eine marginale Rolle ein. Die Adressierung wird in diesem Fall zwar nicht zu Beginn des Interviews explizit, die Erwartungen Herrn Tanners an das Interview können jedoch durch das Anschreiben der Forscherin vorstrukturiert sein. Die Tatsache, dass Herr Tanner sowohl das Interview einläutet, als auch die Interviewsprache festlegt, spricht aber dafür, dass er dem, was er zu sagen hat, eine hohe Relevanz zuspricht. Auf die »verspätete« Einstiegsfrage hin gibt er sich als Gesellschaftskritiker, womit er seine Expertise im Kontext von Jugendgewaltprävention reformuliert. Darüber hinaus hat er ein Verständnis davon, wie Intervention und Beziehungsarbeit gut gelingen können. Dabei verweist er auf seine berufsbiografische Erfahrung, die eine klare Rolleneinteilung zwischen »guten« und »bösen« Jugendarbeiterinnen bzw. Jugendarbeitern relevant setzt. Herr Tanner adressiert die Interviewerin als Soziologin und zeigt das Bestreben an, »soziologischem « Wissen gerecht zu werden bzw. stellt er seine Beiträge zumindest in einen Vergleich zu »soziologischem « Wissen. Dies spricht dafür, dass er die Interviewerin als Expertin einer anderen Wissenskultur oder aber auch als Co-Expertin anerkennt. Dennoch schildert er seine berufsbiografischen Erfahrungen, vor deren Hintergrund er »gute« Jugendarbeit expliziert. Auch sprachlich strebt er eine gemeinsame Ebene an. Herr Tanner differenziert aus seiner fachbereichsspezifischen Erfahrung heraus zwischen »Intervention« und »Wiedergutmachen« und damit zwischen »guten « und »bösen« Jugendarbeiterinnen. Dieses Spannungsfeld sei für Jugendarbeit zentral. Von Jugendarbeit im Sinne einer Beziehungsarbeit distanziert er selbst sich aber. Stattdessen expliziert er seine gesellschaftskritische Position im Kontext von Jugendgewaltprävention. Seiner Perspektive nach ist die gesellschaftliche Dominanz des Sprechens über Jugendgewalt »Gewalt an der Jugend «. Die Legitimität, über »Jugendgewalt« zu sprechen, erfordere das Sprechen über die Gewalt anderer Personengruppen. (Zur Erinnerung: Folgende fallbezogene Zusammenfassungen beziehen sich auf die Interviews, die mit einer offenen Erzählaufforderung geplant worden sind.)

\section{Frau Blaum, Jugendarbeiterin}

Frau Blaum, die im Interaktionsverlauf des Interviews nicht direkt als Expertin für Jugendgewaltprävention adressiert wird, erzählt offen über ihre Tätigkeit als Jugendarbeiterin, wobei sie einen Bruch zwischen ihrem Selbstverständnis und der von außen an sie herangetragenen Erwartungen markiert. Den Auftrag ihrer Arbeitgeberin, der Stadt, Jugendgewaltprävention zu »erfüllen«, hält sie aufgrund der restriktiven Bedingungen ihrer Einrichtung, die insbesondere in der Realisierung der durch die im Quartier lebenden Familien und Jugendlichen formulierten Nachfragen besteht, für nicht realisierbar. Insofern distanziert sie sich in ihrer Expertise von spezifischer Jugendgewaltprävention und beschreibt ein Dilemma, das in der Unvereinbarkeit unterschiedlicher 
»Aufträge « besteht. Schließlich fällt auf, dass Frau Blaum die Jugendlichen, die ihre Einrichtung besuchen, als »Expertinnen« bzw. »Experten« re-adressiert, indem sie ihnen einen Teil der Verantwortung zuspricht, »gute« Jugendarbeit zu realisieren. Frau Blaum beschreibt ihren Tätigkeitsbereich ausführlich und lässt sich auf Nachfragen ein. In dieser Beschreibung entwickelt sie das Ideal ihrer Arbeit, das mit den Erwartungen ihrer Auftraggeberin hinsichtlich Prävention bricht. Die offene Erzählaufforderung ermöglicht ihr, ihre eigenen Relevanzen, die primär in der Erfüllung eines Ideals liegen, zu entwickeln sowie aufzuzeigen, dass sie in dem Bestreben, ihr Ideal zu erfüllen, den Erwartungen ihrer Auftraggeberin hinsichtlich Jugendgewaltprävention nicht gerecht werden kann. Frau Blaum situiert ihre fachbereichsspezifische Tätigkeit in ein Spannungsverhältnis zwischen dem durch die Stadt formulierten Präventionsauftrag und den Interessen der Jugendlichen sowie den im Quartier lebenden Familien. Beiden Interessen gerecht zu werden, sei nicht möglich. Dabei macht Frau Blaum ein Präventionsverständnis implizit, das auf Nachhaltigkeit beruht. Dieses kann sie nicht erfüllen, da es von den restriktiven Bedingungen ihrer Einrichtung (Freiwilligkeit, Beziehungsarbeit, Vertrauensarbeit, Nachfragen, fehlende Informationen über die jeweiligen Lebenslagen der Jugendlichen), in die sie ihr Ideal situiert bzw. aus denen ihr Ideal resultiert, begrenzt wird. Vor diesem Hintergrund problematisiert sie Jugendgewaltprävention insofern sie infrage stellt, dass es möglich ist, präventiv zu handeln, wenn Gewalt da ist. Prävention als nachhaltige Aufgabe erfordere Wissen (über die familiäre Situation und das Freizeitverhalten der Jugendlichen), über das sie aus ihrem Handlungskontext heraus nicht verfüge. Gleichzeitig stehe sie jedoch in der Pflicht, der Auftraggeberin über ihre Präventionsarbeit Zeugnis abzuliefern. Frau Blaum entwickelt ihre Expertise in der Orientierung am Idealzustand ihrer Arbeit: gemeinsam Freizeit verbringen. Dieser Zustand schließt mit ein, dass die Jugendlichen dazu bereit sind, ihre Bedürfnisse zu äußern. In dieser Hinsicht schreibt Frau Blaum einen Teil der Expertise, der ihre Arbeit auszeichnet, den Jugendlichen zu. Sie sind aus Frau Blaums Perspektive Verbündete - Co-Expertinnen bzw. Co-Experten -, die in einem begrenzten Raum agieren, der auch als Schutzraum angesehen werden kann. Innerhalb dieses Raumes reagiert Frau Blaum auf die Bedürfnisse der Jugendlichen.

\section{Herr Auto, Geschäftsführer einer privaten Organisation}

Herr Auto wird in der offenen Erzählaufforderung direkt als Experte für Jugendgewaltprävention adressiert. Von dieser Zuschreibung distanziert er sich zwar nicht direkt, stellt aber dar, dass seine Organisation im Prinzip keine Unterscheidung zwischen Prävention und Intervention macht, sondern auf konkrete Nachfragen reagiert. Seine Expertise gründet demnach nicht in Jugendgewaltprävention, sondern er begründet sie betriebsökonomisch: Seine Organisation reagiere auf Nachfragen jeglicher Art, wobei er als »Generalist« 
einen Vorteil gegenüber anderen Organisationen habe. Ziel seiner Organisation sei es, eine wachsende Nachfrage bezüglich Jugendgewaltprävention zu befriedigen. An dieser Nachfrage sei er berufsbiografisch gewachsen. Es fällt auf, dass Herr Auto seine Organisation, deren Ziele und seine eigene Berufsbiografie, die in einem engen Zusammenhang stehen, stark rechtfertigt, was ein Hinweis darauf sein könnte, dass er die Interviewerin als potenzielle Kritikerin wahrnimmt, aber auch darauf, dass er seine Wissenskultur für begründungspflichtig hält. Herr Auto begründet die Ziele seiner Organisation, die unmittelbar mit seiner beruflichen Biografie verwoben sind, mit der wachsenden Nachfrage an Jugendgewaltprävention, insbesondere an Generalisten auf diesem Gebiet. Er reflektiert seine Arbeit nicht über Deutungen von Jugendgewalt. Vielmehr sieht er seine Organisation und seine Expertise an einer wachsenden Nachfrage nach Prävention und Intervention begründet.

\section{Herr Ifon, Leiter der Jugendpolizei einer Stadt}

Herr Ifon distanziert sich von der Zuschreibung als Experte für Jugendgewaltprävention, indem er unmittelbar angibt, nicht Prävention stehe im Fokus dieses Bereichs, sondern Ermittlung. Damit rekonstruiert er seine Expertise in Abgrenzung zu Jugendgewaltprävention, aber auch in Abgrenzung zur Ermittlertätigkeit, insofern er angibt, die meiste Zeit vom Schreibtisch aus zu arbeiten. Die Informationen, die über seinen »Tisch gehen«, vermitteln ihm jedoch einen Überblick. Herr Ifon beschreibt die Tätigkeit des Jugenddienstes der Stadtpolizei und den für ihn relevanten Tätigkeitsbereich sehr ausführlich. Dabei schreibt er seiner Biografie sowohl beruflich als auch privat eine hohe Relevanz bezüglich seiner Expertise zu. Herr Ifon vermischt verschiedene Sprecherpositionen miteinander. Er spricht gleichzeitig als Vertreter einer »älteren Generation« und als Leiter des Jugenddienstes, der zwischen der »älteren« Generation und den Jugendlichen vermitteln muss. Herr Ifon hält es zunächst für relevant, seine biografische Entwicklung zu schildern, in der er private und berufliche Aspekte miteinander verbindet. Er leitet nicht nur den Jugenddienst, sondern vertritt auch die Interessen der »älteren Generation«. Aus dieser Vermittlerposition ergeben sich mögliche Interessenkonflikte. Mögliche Interessenkonflikte könnten hier eine Rolle spielen, insofern er als Leiter des Jugenddienstes zwischen den Interessen der »älteren Generation« und den Jugendlichen vermitteln muss. Er stellt klar, dass Jugendgewaltprävention nicht primär Bestandteil von Jugendpolizeiarbeit sein kann und sein Tätigkeitsbereich viel weiter reiche, da er jegliche strafbaren Verhaltensweisen Jugendlicher zum Gegenstand hat. Jedoch sieht er Strafe auch als Prävention in dem Sinne an, als sie vorbeugend wirken kann. Herr Ifon differenziert implizit zwischen »normaler« (»Hängen«, »Alkohol trinken« und »Littering«) und »pathologischer« Jugendgewalt. Letztere werde von fünf Prozent der Jugendlichen ausgeübt und zeichne sich durch die Intensität eigentlich »norma- 
len« jugendlichen Gewaltverhaltens aus. Herrn Ifons »Sorge« gilt also primär einer bestimmten Personengruppe.

\section{Herr Schäfer, Jugendrichter}

Herr Schäfer ist sehr darum bemüht, seinen Erfolg, den er seiner »Philosophie« zuschreibt, glaubhaft darzustellen. Aus den Schilderungen seines Tätigkeitsbereichs geht der Anspruch, glaubhaft wahrgenommen zu werden, besonders deutlich hervor. Dies könnte dafür sprechen, dass er die Interviewerin als potenzielle Kritikerin ansieht. Bestärkt wird dieser Eindruck außerdem durch die Thematisierung der Interviewsprache (Hinweis auf die ausländische Herkunft der Interviewerin) und die nachfolgende Herausstellung der Besonderheit des schweizerischen Jugendstrafrechts. Als nicht unbedingt problematisch, aber besonders relevant für die Jugendanwaltschaft bezeichnet Herr Schäfer die Verwobenheit von Jus, Sozialarbeit und Sozialpädagogik - Perspektiven, zwischen denen er als Jugendanwalt pendeln muss. Gewalt beschreibt er als »Delinquenz« Jugendlicher, die in Grenzüberschreitungen besteht. Diese normalisiert er und differenziert sie von Grenzüberschreitungen, die »pathologisch « sind und sozialen und kulturellen Prozessen unterliegen. Die Frage nach den Ursachen ist für ihn relevant, aber auch die Notwendigkeit, Gewalt zu messen und zu differenzieren, da er über die Unterbringung in Maßnahmen entscheiden muss.

Neben der Strategie des »(un-)doing expertise«, die in der Relativierung einer zugeschriebenen Expertise besteht und der hier besondere Aufmerksamkeit gewidmet wird, zeigen sich weitere gemeinsame Muster und Auffälligkeiten, die in den weitergehenden, primär themenbezogenen Interpretationen berücksichtigt werden. Die Positionierungen der Befragten zeichnen sich durch Sprechrollenwechsel aus: Die Perspektiven beziehen sich zum Teil abwechselnd auf professionelle Kontexte, berufsbiografische Erfahrungen, persönliche Standpunkte, wissenschaftliches Wissen, Gesellschaftskritik sowie Erfahrungen im privaten Leben. Insbesondere wissenschaftliches Wissen und berufsbiografische Erfahrungen fungieren dabei als zentrale Deutungsmuster, anhand derer Jugendgewalt problematisiert wird: Dabei wird die Normalisierung von Jugendgewalt (hauptsächlich hinsichtlich des Geschlechts, der Kultur, biologischer Voraussetzungen und der historischen Entwicklung) einer Skandalisierung bzw. Pathologisierung von Jugendgewalt (in Bezug auf die Intensität, Häufigkeit und Dauer) gegenübergestellt. Hinsichtlich der Prävention zeigen sich ambivalente Positionierungen zwischen den Erwartungen von »außen« (v.a. in Form von Aufträgen) und den eigenen Relevanzsetzungen. Darüber hinaus fällt ein Rechtfertigungs- und Erfolgsdruck nach »außen« auf, der sich auch in der Interviewsituation widerspiegelt bzw. auf diese projiziert wird. Im Folgenden geht es v.a. um die Frage, inwieweit diese Beobachtungen 
etwas über das Feld der Prävention erzählen oder aber dem Erhebungsinstrument geschuldet sind. Die vorliegende Arbeit, die sich diskursanalytisch an den Gegenstand der Jugendgewaltprävention annähert, sieht Interviews als diskursive Praktiken an, die in einem bestimmten Kontext und somit Möglichkeitsraum des »Sprechens über« situiert sind. Dabei geben Deutungsmuster von Jugendgewalt Aufschluss über (Un-)Möglichkeiten von Sprechen über Jugendgewaltprävention als Praktik. Deutungsmuster bzw. Orientierungsmuster im Sinne der dokumentarischen Methode sind nicht explizit erfassbar, sondern werden in der Interviewpraxis rekonstruiert (vgl. Bogner/Menz 2009) - eine Leistung, die aus einer an sozialen Praktiken interessierten diskursanalytischen Perspektive vor dem Hintergrund gegenseitiger Erwartungshaltungen der am Interview Beteiligten reflektiert werden muss. Dafür spricht auch die Beobachtung, dass die Interviewten den durch die Interviewerin zugeschriebenen Status einer Expertin bzw. eines Experten relativieren und sich den Fragen auf unterschiedliche Weise entziehen. Dabei wird angedeutet, dass Jugendgewaltprävention ein wenig konkretisierbarer und kein besonders relevanter Bestandteil der jeweiligen Fachbereiche ist. Der Frage, inwiefern die Beobachtung der Relativierung oder zumindest Distanzierung von Zuschreibungen hinsichtlich der Expertise der befragten Fachpersonen durch diese als dem Kontext (Jugendgewaltprävention) eigentümlich angesehen werden kann oder der Adressierungsweise als Expertin bzw. Experte im Allgemeinen geschuldet ist (Erhebungsinstrument), nähert sich die hier vorliegende Arbeit, indem sie das Interview als Interaktion betrachtet, die insbesondere von den Erwartungshaltungen der am Interview beteiligten Personen strukturiert ist und dahingehend zu interpretieren ist. Auch diesbezügliche Rekonstruktionsweisen sind dahingehend $\mathrm{zu}$ interpretieren. Demnach ist der Beobachtung Rechnung zu tragen, dass die »(Re)Konstruktion des Deutungswissens im Experteninterview in Abhängigkeit von der Interaktionsstrategie immer (nur) einen bestimmen Ausschnitt aus dem Expertenwissen realisiert« (ebd.: 94). Die Interpretationen weisen in der Tendenz darauf hin, dass die Befragten der offenen Aufforderung, über ihre Tätigkeit zu erzählen, nachkommen. Dies ermöglicht ihnen, ihre jeweiligen Expertisen und damit einhergehenden Problematisierungsweisen nach jeweils eigenen Relevanzsetzungen zu entwickeln, da sie sich weniger an einer ihnen zugeschriebenen Figur abarbeiten müssen. Nichtsdestotrotz lassen sich auch hier Muster der Begründung sowie Problematisierungen des Erfolgsdrucks nach außen bzw. der erwarteten Kritik durch die Forscherin beobachten, was der Adressierung durch das Anschreiben oder aber dem Feld geschuldet sein könnte. Aus den Interviews geht hervor, dass die Interviewerin in der Tendenz als »Vertreterin einer anderen Wissenskultur« oder aber als »Laie« adressiert wird. Die damit verbundenen Erwartungen fördern die Explikation der jeweils eigenen Orientierungsrahmen, vor deren Hintergrund auch die jeweiligen Relevanzen, Problematisierungsweisen und 
Deutungsmuster von Gewalt bzw. Jugendgewalt entwickelt werden. Somit lässt sich behaupten, dass die hier vorliegenden »Experteninterviews« theoriegenerierenden Interviews nahekommen, insofern als sie die Rekonstruktion von Deutungswissen zulassen,

"also jenen subjektiven Relevanzen, Regeln, Sichtweisen und Interpretationen des Experten, die das Bild vom Expertenwissen als eines heterogenen Konglomerats nahelegen. Mit der Rekonstruktion dieses ,Deutungswissens، betritt man - altmodisch formuliert - das Feld der Ideen und Ideologien, der fragmentarischen, inkonsistenten Sinnentwürfe und Erklärungsmuster. Mit dieser analytischen Differenzierung wird zum einen das spezifische Erkenntnisinteresse des theoriegenerierenden Experteninterviews präziser darstellbar. Zum anderen wird auf diese Weise deutlich, dass das Expertenwissen als 'homogener Wissenskörper nicht hinlänglich zu fassen ist." (Ebd.: 72)

Demnach ist davon auszugehen, dass die Expertin bzw. der Experte für Jugendgewalt nur als Figur fungiert, an deren Grenzen die eigene Expertise konstruiert und damit rekonstruierbar wird. Wissen kann nicht per se als gültig anerkannt werden, sondern ist nur innerhalb eines je spezifischen Orientierungsrahmens (re-)konstruierbar, der in der vorliegenden Arbeit über die Figur der Expertin bzw. des Experten mit auf kriminalpolitischer Ebene relevant gesetztem Wissen kontrastiert wird. Außerdem zeigt sich die Tendenz, dass die Befragten Rechtfertigungsdruck von außen äußern und ihre »Präventionsarbeit« deshalb durch die Schilderung von Erfolgserlebnissen oder -konzepten absichern. Dies impliziert, dass eigentlich alles gut und nichts zu hinterfragen sei, und verweist womöglich bereits auf den kriminalpolitischen Diskurs über Jugendgewaltprävention im Rahmen des »Nationalen Präventionsprogramms Jugend und Gewalt«, das insbesondere danach strebt, Prävention evident bzw. sichtbar zu machen, womit ein gewisser Wunsch nach Kontrolle und Wissen einhergeht. Die Relativierung des durch die Interviewerin zugeschriebenen Status einer Expertin bzw. eines Experten, was hier im Sinne einer interaktiven Praxis der Interviews als »(un-)doing expertise « bezeichnet wird, ist den Befragten weitgehend gemeinsam. Nun stellt sich die Frage, inwiefern diese Praxis dem Kontext des Sprechens über Jugendgewaltprävention im weiteren Sinne geschuldet ist oder der Form des Interviews, was im Folgenden unter theoretischen und methodischen Gesichtspunkten diskutiert wird. Es kann angenommen werden, dass die Interaktionssituationen der Interviews durch gegenseitige Zuschreibungen und Erwartungen strukturiert sind, was einer Reflexion unterzogen werden muss, u.a. um die Interviews hinsichtlich ihrer Güte bezüglich des jeweiligen Forschungsanliegens zu beurteilen (vgl. Bogner/Menz 2009; Helfferich 2011: 58f.). Gerade aus diskursanalytischer Perspektive ist diese Reflexion zentral, da davon auszugehen ist, dass das »eigene Relevanzsystem, von dessen Basis aus Verstehen geschieht« (Helfferich 2011: 
58), durch diskursives Wissen vorstrukturiert ist. Das Ziel der hier vorliegenden Arbeit ist die Rekonstruktion von Deutungswissen anhand der Beschreibung subjektiver Erfahrungs- und Handlungsräume und die Beantwortung der Frage, inwieweit dieses Wissen vor dem Hintergrund eines kriminalpolitischen Diskurses konstruiert wird. Die bisherigen Interpretationen weisen darauf hin, dass die direkte Adressierung als Expertin bzw. Experte für Jugendgewalt am Beginn des Interviews, die Notwendigkeit verstärkt, damit einhergehende Zuschreibungen zu korrigieren. Dies bietet prinzipiell Möglichkeiten der Rekonstruktion von Deutungswissen hinsichtlich der Zuschreibungen, die sich eines Diskurses über Jugendgewaltprävention bedienen und diesen damit reproduzieren. Gleichzeitig bietet dies Möglichkeiten der Rekonstruktion der Expertise, welche die Befragten sich selbst im Kontrast zu dem zugeschriebenen Status einer Expertin bzw. eines Experten zuweisen. Die unmittelbare Adressierung der Befragten als Expertinnen bzw. Experten für Jugendgewaltprävention ermöglicht ihnen, sich sowohl auf diskursives Wissen zu beziehen, als auch, in Kontrast dazu, die eigenen jeweils spezifischen Bedeutungsräume zu eröffnen. Wie die Interpretationen zeigen, birgt diese Vorgehensweise allerdings auch das Risiko, dass die Interviewfragen nicht ernst genommen werden oder sich die Befragten von Schilderungen über spezifische Aspekte ihres Fachbereichs distanzieren. Herr Gold und Herr Vonderstatt distanzieren sich auf ironische Weise von den Fragen der Interviewerin. Sie präsentieren sich in ihrer Expertise zunächst ausschließlich als Gesellschaftskritiker bzw. »Meinungsgeber« und rechnen sich eine gewisse Deutungshoheit zu. Einblick in ihr berufliches Relevanzsystem verwehren sie zunächst. Herrn Vonderstatts Strategie, im weiteren Interviewverlauf eigene Fragen zu formulieren, diese zu beantworten und die Fragen der Interviewerin weitgehend zu ignorieren, könnte auch darauf hinweisen, dass er keine gehaltvollen Informationen preisgeben will, möglicherweise aus der Erwartung heraus, die Interviewerin könnte hinsichtlich ihrer Fragen von kritischem oder evaluativem Interesse geleitet sein. Herr Unterwasser und Frau Schock hingegen grenzen sich zunächst von Zuschreibungen ab und führen in ihren je eigenen Erfahrungshorizont ein, dem sie ihre Expertise zurechnen. Dabei distanzieren auch sie sich in gewisser Weise von der Zuschreibung als Expertin bzw. Experte für Jugendgewaltprävention, insofern sie sich von der Arbeit mit Jugendlichen distanzieren. Frau Schock arbeitet mit und in Familien, also dort, wo sie und ihre Auftraggeberinnen bzw. Auftraggeber den Ursprung von Jugendgewalt vermuten. Herr Unterwasser arbeitet mit Erwachsenen, an die er die Arbeit mit Jugendlichen delegiert. In dem Interview mit Herrn Tanner zeigt sich, dass die Adressierung, die im Anschreiben erfolgt, bei den Befragten möglicherweise eine so hohe Erwartungshaltung auslösen kann, dass sie die Einstiegsfrage gar nicht erst abwarten. Im Interviewverlauf zeigt sich zudem, dass Herr Tanner sich schriftlich auf das Interview vorbereitet und sogar 
bereits eine Art Schlussplädoyer verfasst hat. Er sieht, ebenso wie Frau Schock und Herr Unterwasser, die Interviewerin zwar als Expertin einer anderen Wissenskultur an, will dieser aber auch gerecht werden, indem er sich als Gesellschaftskritiker darstellt (ohne sich ironisch zu distanzieren) und sich dabei auf »soziologisches« Wissen bezieht. Von dieser Darstellung grenzt er jedoch seine Expertise ab, indem er die Bedeutung seiner berufsbiografischen Entwicklung in der offenen Jugendarbeit beschreibt. Durch die offene Einstiegsfrage werden die Befragten darin bestärkt, die jeweils spezifischen Erfahrungs- und Handlungsräume sowie die eigenen Expertisen zu beschreiben. Frau Blaum, Herr Auto und Herr Ifon schildern ihren Tätigkeitsbereich ausführlich und lassen sich ernsthaft auf Nachfragen ein. Herr Schäfer hingegen nimmt die Fragen der Interviewerin weniger ernst, stattdessen schildert er sein persönliches Erfolgsrezept. Welche Interviews können nun für die Analyse als besonders geeignet angesehen werden? Herausfordernd ist die Erkenntnis, dass die Befragten über die Adressierungsweisen und Zuschreibungen der Forscherin zu Expertinnen bzw. Experten gemacht werden, da die Forscherin diese in einen bestimmten Kontext situiert, den sie für ihre Fragestellung als relevant erachtet (vgl. Bogner/Menz 2009: 68f.). Die bisherigen Interpretationen zeigen, dass die Befragten zwar tendenziell an die Zuschreibungen anschließen können, sich aber gleichzeitig kritisch davon distanzieren und sich in der Darstellung ihrer Expertise davon abgrenzen. Dadurch wird implizites Wissen rekonstruierbar. Deshalb fokussieren die folgenden Analysen zunächst auf die Interviews, in denen die Fragen der Interviewerin ernst genommen werden und/oder die Forscherin als Expertin einer »anderen Wissenskultur« angesehen wird. Die Interaktionsstruktur kann sich jedoch im Verlauf des Interviews ändern, worauf die Interpretationen der Einstiegspassagen teilweise hindeuten (vgl. ebd.: 81). ${ }^{1}$ Ein weiteres Kriterium der Fallauswahl betrifft die Relevanz von Jugendgewaltprävention, wobei diejenigen Interviews als zentral erachtet werden, in denen Jugendgewaltprävention als verhältnismäßig wichtig für das jeweilige Feld angesehen wird. Schließlich werden diejenigen Interviews bzw. Interviewsequenzen berücksichtigt, in denen die Befragten offen über ihre Deutungs- und Handlungskontexte erzählen und die damit auch eine hohe narrative Dichte aufweisen. In diskursanalytischen Vorgehensweisen stehen Forschende unter dem Zwang, ihre Positionierung bezüglich des von ihnen beforschten Gegenstandes kritisch zu reflektieren (vgl. Wedl/Wrana 2014:

1 Einige Sequenzen zeigen auf, wie die Teilnehmenden der Interviews über Wissen verhandeln, indem sie sich an die jeweilige andere Wissenskultur annähern bzw. sich davon distanzieren. Insgesamt ist der Gewinn der hier vorliegenden Interaktionssituationen, in denen sich die Teilnehmenden als Expertinnen bzw. Experten einer anderen Wissenskultur begreifen, für "theoriegenerierende bzw. deutungswissenschaftliche Ansätze" (ebd.: 82) als hoch einzuschätzen. 
479). Dies gilt insbesondere für diskursanalytische Vorgehensweisen, die sich Praktiken der Interviewforschung bedienen. Sie kommen nicht umhin, eben jene diskursiven Muster zu gebrauchen, die sie eigentlich beforschen. Die Notwendigkeit dieser Reflexion wird in der Interviewpraxis besonders virulent, gilt aber auch für andere Forschungsmethoden, die nicht unbedingt an diskursanalytische Vorgehensweisen gebunden sind, wie auch Stefan Hirschauer für die Ethnografie expliziert. Diese könne die »Schweigsamkeit des Sozialen« (Hirschauer 2001) versprachlichen und erfasse somit eben diejenigen Relevanzen, die Transkriptionen ausblenden. In gewisser Weise lässt sich behaupten, dass aus der Not eine Tugend wird. Selbstreflexion meint dabei nicht den Versuch, den Einfluss der Forschenden auf den Erhebungsprozess auszuschalten und vermeintliche »Objektivität« herzustellen, sondern diesen Einfluss analytisch zu nutzen - »die von der Interviewerin angebotenen Subjektpositionen methodisch zu nutzen « (Bender/Eck 2014: 487). So beurteilt Daniel Wrana diskursanalytische Arbeiten, in diesem Fall von Hans-Christoph Koller und Sabine Reh in einer Diskussion »Zur (Selbst-)Reflexivität des Forschungsprozesses«, folgendermaßen:

"Anders als in der qualitativen Forschung üblich, versuchen sie nun nicht, ihren eigenen Einfluss auf die InterviewpartnerInnen ungeschehen zu machen oder zu separieren, sondern sie betrachten die biographischen Narrationen als Rechtfertigungen und Rekonstruktionen gegenüber einem in einer bestimmten Weise positionierten Fragenden. Reflexiv wird die Interviewsituation als diskursive Produktionspraxis und nicht der Interviewer oder die Interviewerin als Person. Weder Koller noch Reh kombinieren , die Diskursanalyse، mit , dem narrativen Interview، oder gar 'der Biographieforschung', sie rekonstellieren sie und verändern sie dabei grundlegend. Das narrative Interview ist nicht mehr, was es in der Biographieforschung war." (Feustel et al. 2014: 498f.)

Die vorliegenden Interpretationen zeigen den reproduktiven Gebrauch diskursanalytischer Figuren einer Expertin bzw. eines Experten durch die Praxis des Interviews, wobei eben genau diese zum Gegenstand der Analyse werden. Die Relativierung von Zuschreibungen dieser Figuren durch die Befragten gleicht einer Dekonstruktion diskursiv etablierter Sinnmuster und verweist somit auf Transformationsprozesse zwischen der kriminalpolitischen Programmatik und Handlungspraktiken. Gleichzeitig werden praxisrelevante Deutungen konstruiert, die mit diskursiv erzeugten Orientierungsmustern brechen und in weiteren Interpretationen vertiefend verfolgt werden. Subjekttheoretisch bzw. diskurstheoretisch - der Subjektbegriff kann im Anschluss an Foucault als der Diskurstheorie inhärent begriffen werden - lassen sich in den zugrunde liegenden Interviews Zuschreibungen beobachten, die den Befragten Möglichkeiten zuweisen, bestimmte Subjektpositionen und Subjektivierungsweisen einzunehmen. Es lässt sich sogar sagen, dass Zuschreibungen Selbstpositio- 
nierungen gewissermaßen erzwingen, wodurch sich Subjektivierungsweisen rekonstruieren lassen (vgl. Bender/Eck 2014: 437). Die unterschiedlichen Strategien, mittels derer sich die Befragten zunächst von Zuschreibungen distanzieren, diese damit dekonstruieren und sich schließlich dazu positionieren und ihren Expertenstatus somit rekonstruieren, sind als ein Hinweis auf die Widerständigkeit von Subjekten zu deuten: Das Subjekt ist demnach nicht nur ein »diskursiver Effekt, eine diskursiv hergestellte Illusion innerer Einheit« (Angermüller/Wedl 2014: 167). In der vorliegenden Arbeit fungiert das Subjekt vielmehr als analytische Kategorie, die Einsicht über Transformationen des Verhältnisses von Politik und Sozialer Arbeit gewährt. An der Problematisierung und Reflexion der von der Forscherin zunächst gewählten Einstiegsfrage nach dem »Kernproblem von Jugendgewalt « wird deutlich, wie stark die Forscherin selbst Teil eines Diskurses über Jugendgewalt ist: Jugendgewalt wird diskursiv skandalisiert und problematisiert, jedoch wird gleichzeitig in Aussicht gestellt, dass damit einhergehende Probleme durch verschiedene Fachbereiche bearbeitbar seien und bearbeitet werden sollen (vgl. Stehr 2009: 110). Die folgenden Interpretationen (4.1.2 und 4.1.3) erfolgen also nicht primär themenspezifisch, sondern fall- und themenspezifisch, vergleichend und auf Basis der Deutungen, die anhand der bisherigen Interviews rekonstruiert worden sind. Die relevanten Themen werden also im Fallvergleich erarbeitet. Bei der Auswahl vergleichend $\mathrm{zu}$ interpretierender Interviewsequenzen werden Sequenzen mit »hoher narrativer Dichte« ausgewählt, die also im Sinne der dokumentarischen Methode »einen hohen Detaillierungsgrad « haben, und »den Charakter von Fokussierungsmetaphern annehmen« (Bohnsack 2001: 233f.). Auch aus der Perspektive der Diskursforschung weisen diese Sequenzen eine hohe Relevanz auf, insofern als sie Narrationen als diskursive Praktiken auffassen:

„Für Diskursforschende, die daran interessiert sind, Produktionsweisen und Praktiken der Subjektivierung zu untersuchen, sind Narrationen hoch anschlussfähig, insbesondere weil auch die Interviewsituation stets als diskursive Situation zu begreifen ist: Sie funktioniert sprachlich, sie läuft über Positionierungen, in ihr werden Subjekte in diskursive Ordnungen rekrutiert." (Bender/Eck 2014: 476)

Reiner Keller spricht hier von sog. »Story Lines« (Keller 2009: 46), die sich vom Interpretationsrepertoire dahingehend unterscheiden, dass sie nicht auf die gesamten Grundannahmen eines Diskurses, sondern auf die für ein je spezifisches Publikum relevanten verweisen. Neben der ambivalenten Positionierung hinsichtlich der Expertise über Jugendgewalt bzw. Jugendgewaltprävention fällt ein weiteres Muster auf: das Pendeln zwischen Bedrohungsszenarien auf der einen Seite und der Normalisierung von Jugendgewalt über verschiedenste Deutungs- und Erklärungsmuster auf der anderen Seite. Damit treffen 
»Normalitätserwartungen« der Fachpersonen, »die darüber Auskunft geben, wann welche Verhaltensweisen als legitime Artikulationen des Eigenwillens von AdressatInnen anerkannt werden und wann gegen sie interveniert werden soll« (Dollinger/Oelkers 2015: 11), auf einen Diskurs, der durch das Spannungsverhältnis zwischen »Dramatisierung « und »Ausblendung « eines Problems charakterisiert ist (Liell 2002: 6). Die Normalisierung von Jugendgewalt ist im Kontext von Jugendgewaltprävention, insbesondere in der Sozialen Arbeit, eine zentrale Strategie, die weiter auszudifferenzieren ist, insofern die Normalitätserwartungen diskursiv vorstrukturiert sind, sich die damit verbundene »Normalitätsarbeit« (Dollinger/Oelkers 2015: 11) an Risiken orientiert und damit auf die Legitimation der Fachbereiche abzielt (4.1.3). Arbeiten, die sich an diskursanalytischen Theorietraditionen orientieren, gehen davon aus, dass auf politischer Ebene angesiedelte Programme Probleme und folglich auch Lösungswege (mit-)konstituieren sowie die für die Umsetzung verantwortlichen Akteure ansprechen - im Sinne Althusserls »anrufen« (Ott/Wrana 2010: 156f.). Sie nehmen also an, dass Programme Subjekte (mit-)konstituieren. Hierbei stellt sich die Frage, inwiefern das »Subjekt« »nur« als erkenntnistheoretische und/ oder auch empirische Kategorie anzusehen ist (vgl. Gehring 2008: 21). Trotz des Rekurses auf Foucault, nach dem unter dem Subjekt vielmehr eine »historically specific mode within which human beings can experience themselves « (Kendall 2011: 70), demnach vielmehr eine historisch kontextualisierte Erfahrungsweise und weniger eine sich in konkreten Situationen konstituierende Seinsweise zu verstehen ist, versuchen aktuelle Forscherinnen und Forscher, Subjekt und Subjektivierungsprozesse empirisch zu erfassen. Die hier vorliegende Arbeit begreift das Subjekt in erster Linie als erkenntnistheoretische Figur, mit der sie sich empirischen Fragen annähert, insbesondere der Frage nach Problematisierungsweisen und daran anschließenden Präventionsverständnissen der befragten Fachpersonen in Bezug auf eine kriminalpolitische Programmatik. Das Interview ist hierbei als diskursive Praktik zu verstehen, die im Sinne

"einer sozial geregelten, typisierten, routinisierten Form des körperlichen Verhaltens (einschließlich des zeichenverwendenden Verhaltens) und darin spezifische Formen des impliziten Wissens, des Know-how, des Interpretierens, der Motivation und der Emotion umfasst. Praktiken unter dem Subjektaspekt zu betrachten, bedeutet zu fragen, in welcher Richtung sie subjektivieren`, d.h. welche Dispositionen eines zugehörigen Subjekts sie nahe legen und über welche Wege innen diese Modellierung eines entsprechenden Körpers, eines Wissens und einer Psyche gelingt." (Reckwitz 2008a: 135)

Anschließend an diese methodologische Reflexion ist die analytische Frage demnach, welche Subjektformen in den geführten Interviews produziert werden. »Subjektformen werden in sozialen Praktiken routinemäßig produziert; 
in Diskursen werden sie explizit zum Thema und in Form von Subjektrepräsentationen hergestellt und gesellschaftlich verfügbar gemacht.« (Ebd.: 137) Dabei muss jedoch klar sein, dass die in der Interviewpraxis vorgenommenen Adressierungsweisen nicht mit Anrufungsprozessen gleichzusetzen sind, also nicht von einem deterministischen Verständnis von Subjektkonstitution auszugehen ist (vgl. Butler 2001: 15; Keller 2010: 48; Reckwitz 2008a: 140). Subjekte konstituieren sich nicht über Adressierung und sind demnach nicht als »Personen« zu verstehen, sondern sie konstituieren sich über Anrufungsprozesse. Die Konzepte sind sich jedoch durchaus ähnlich, insofern beide

"Prozesse der Zuschreibung und solche der auf diese reagierenden Selbstkonstitution verbinden. [...] Anders ausgedrückt: Kommunikation benötigt und produziert Akteursfiktionen, und es ist diese ,kommunikative Verfertigung von Akteuren ، als Zurechnungspunkte, auf die das Konzept der Adressierung bzw. Adressabilität abhebt. Akteur ist, auf wen Handeln, insbesondere Mitteilungshandeln zugerechnet werden kann. Kommunikative Prozesse disponieren darüber, wer oder was als soziale Adresse in Frage kommt und wer oder was nicht." (Bröckling 2013: 55)

Über die Reflexion von Adressierungsweisen in der Interviewpraxis nähert sich die hier vorliegende Arbeit dem Subjekt im Sinne von Subjektpositionierungen und -formierungen empirisch an, indem sie Interviews als Interaktionen betrachtet, in denen Zuschreibungen wechselseitig und mit Verweisen auf verschiedene Diskurszusammenhänge erfolgen. Als solche werden sie aufgegriffen, relativiert, reformuliert und damit re-adressiert. Die Frage nach den Interaktionsverläufen geht also über die Frage, wer als Adressatin bzw. Adressat infrage käme, hinaus: Die in den Interviews erfolgenden (Re-)Adressierungen enthalten Anrufungen, die sich als solche nur dann erschließen lassen, wenn die Interviews als Interaktionen interpretiert werden. Anhand der Interpretationen der Eingangssequenzen lassen sich Relativierungen von Zuschreibungen beobachten, was auf einen normativen Deutungsrahmen hinweist, der Subjektpositionierungen hinsichtlich einer in der Adressierung enthaltenen Anrufung hervorbringt, wie etwa: »Weil Sie Expertin für Jugendgewaltprävention sind, wissen Sie, wie man gute bzw. wirksame Präventionsarbeit macht!« Auf diese Anrufung, die Wissen über gute bzw. wirksame Präventionsarbeit enthält, können sich die Befragten auf unterschiedliche Weise beziehen (vgl. König 2008: 4790). Es fällt auf, dass diejenigen Befragten, die ihre Expertise in Abgrenzung dazu deutlich reformulieren und an ihre fachbereichsspezifischen Erfahrungen binden, gleichzeitig auf einen Erfolgs- und Legitimations- bzw. Anerkennungsdruck ihrer Arbeit nach außen verweisen. Daran wird deutlich, dass Subjektpositionierungen über die Orientierung an diskursivem Wissen erfolgen, das in der Interviewsituation relevant wird: Die Adressierungen enthalten Anrufungen, die im diskursiven Kontext der Jugendgewaltprä- 
vention, und damit auch auf kriminalpolitischer Ebene, reproduziert werden und anschlussfähig für Selbstpositionierungen sind. Unabhängig von dieser Beobachtung zeigt sich, dass die Befragten andere, im jeweiligen Kontext agierenden Akteure (re-)adressieren: Fachbereiche, Institutionen, Organisationen und Personengruppen. Herr Unterwasser schildert beispielsweise, dass Erwachsene und andere Organisationen ihn als Experten adressieren. Jugendliche hingegen lehnen die Arbeit mit ihm ab, was seine Distanzierung zur Arbeit mit Jugendlichen wiederum plausibilisiert. Frau Schock benennt und kritisiert Institutionen der Erziehung und Bildung, die sie als Expertin adressieren und sie dabei mit »falschen « Erwartungen belasten. Herr Auto verweist auf den »Markt«, der ihn als Experten adressiert, wodurch er sich in seiner Profession bestätigt sieht. Frau Blaum re-adressiert Jugendliche als Expertinnen bzw. Experten. Diese würden einen großen Teil der Verantwortung für gelingende Jugendarbeit tragen. Herr Vonderstatt, Herr Gold und Herr Tanner zeigen sich zunächst über Adressierungsweisen erhaben, was als Distanzierung zu normativen Deutungsrahmen gelesen werden kann. Wird das Interview als Interaktionsgeschehen in seinem Verlauf beobachtet, kommen zunehmend Re-Adressierungen in den Blick. Anrufung unterscheidet sich von Adressierung dahingehend, dass es sich um ein »reiteratives Verfahren« (vgl. Butler 2006: 54) handelt:

"Folgt man jedoch den Argumentationen von Hall und Butler, so wiederholen sich die Szenarien nicht identisch, sondern zitieren einander im Modus der Iterabilität. [...] Die Anrufung vollzieht sich nicht mit der Adressierung selbst, sondern erst dann, wenn der zweite Akt auf die Adressierung folgt. Und da dieser Akt nicht durch die Adressierung determiniert wird, wird die Anrufung durch dieselbe Adressierung je nach Art und Weise ihrer Beantwortung nicht dieselbe sein." (Ott/Wrana 2010: 165)

Die Re-Adressierung bzw. Reiterierung ist in den vorliegenden Interviewdaten unmittelbar mit der Relativierung verbunden und verweist auf je spezifische Subjektkonstitutionen bzw. Subjektformen als »Expertin« bzw. »Experte«. Im Anschluss daran wird anhand vertiefender Interpretationen herausgearbeitet, welche Deutungen von Jugendgewalt und daran anschließende Präventionsverständnisse die jeweiligen Relevanzsetzungen strukturieren.

\subsubsection{Prävention zwischen Gesellschaftskritik und kriminalpolitischer Programmatik}

Im Folgenden werden mittels vergleichender Interpretationen die zentralen Aspekte der Orientierungsweisen der befragten Fachpersonen an der kriminalpolitischen Programmatik herausgearbeitet. Die zentralen Themen resultieren aus den fallvergleichenden Interpretationen. Fall- und themenbezogene 
Interpretationen greifen somit ineinander, wobei diejenigen Sequenzen in den Fokus geraten, die aufgrund ihrer hohen »narrativen Dichte« als besonders »fokussiert« anzusehen sind. Diese Vorgehensweise dient nicht nur dazu, fallübergreifend die wichtigsten Aspekte zu fokussieren und zu plausibilisieren, sondern auch dazu, die Interaktionsdynamiken der Interviews in den Interpretationen zu berücksichtigen. Die drei zentralen Fälle sind zunächst Herr Tanner, Frau Blaum und Herr Gold. Da Herr Tanner und Frau Blaum in derselben Einrichtung offener Jugendarbeit tätig sind, wobei Frau Blaum als Jugendarbeiterin in einem Quartier und Herr Tanner nach einer Karriere als Jugendarbeiter primär leitend tätig ist, interessiert ein Vergleich aufgrund der Nähe der fachbereichsspezifischen Herausforderungen und Erfahrungen, wobei die unterschiedlichen Funktionen innerhalb der Einrichtung wiederum Kontrastierungsmöglichkeiten eröffnen. Herr Gold agiert als Leiter einer privaten Organisation, die Schulklassen adressiert und Kampfsport unterrichtet, in einem ganz anderen Rahmen. Diese drei Fälle werden entlang zentraler thematischer Aspekte mit weiteren Fällen verglichen. An den vergleichenden Interpretationen zeigen sich zunächst Brüche zwischen den jeweiligen gesellschaftskritischen Positionierungen, aus den jeweils eigenen fachspezifischen Erfahrungen heraus für relevant gesetzte Herausforderungen und jeweilige von außen an die Fachpersonen herangetragene Erwartungen hinsichtlich Prävention. In der bereits einführend interpretierten Einstiegspassage des Interviews mit Herrn Tanner zeigt sich dessen Bestreben, auf die Frage nach dem zentralen Problem von Jugendgewalt, »soziologischem« Wissen gerecht zu werden und damit gesellschaftskritisch zu argumentieren. Er kritisiert den Jugendgewaltdiskurs dahingehend, dass er andere Diskurse, wie etwa über andere »Formen« von Gewalt, überlagert. Diesen Mangel an Differenzierung begreife er als »Gewalt an der Jugend «. Im weiteren Interviewverlauf hält Herr Tanner diese gesellschaftskritische Position nicht aufrecht. Im Sprechen über die für seinen Fachbereich spezifischen Herausforderungen, seine diesbezüglichen Erfahrungen sowie die Leitbilder der Einrichtung setzt er andere Themen relevant. Seine Perspektive oszilliert zwischen zwei Sprecherpositionen, der des Gesellschaftskritikers und der des »leitenden« Jugendarbeiters: Auf der einen Seite hebt er in der Orientierung an der offenen Jugendarbeit, der er zwar biografisch entwachsen ist, die jedoch für seine Leitungsfunktion einen zentralen Hintergrund darstellt, Beziehungsarbeit als grundlegende Voraussetzung für Intervention hervor. Deren Relevanz prägt ein gewisses Selbstverständnis, da Herr Tanner ungefragt und spontan darüber spricht. Auf der anderen Seite argumentiert er gesellschaftskritisch:

Interviewerin: "Und (.) wieso glauben Sie dass dann dieser Begriff Jugendgewalt so stark ist und so präsent auch in in in ja politischen Programmen sozusagen?» 
Herr Tanner: "Ja (.) also ich denke also hab ich meine Thesen ich denke dass ähm Gewalt sich äh gut macht (.) als Thema (.) äh Kriminalität als Thema sich gut macht. (.) Insbesondere (.) wenn man äähm mit wenn man gegen Migrantinnen (.) Gruppen wegen we gegen also ja rassitisch äh lassen wir mal sein aber wenn es darum geht Bevölkerungsgruppe (.) Gruppen anzukreiden darzustellen negativ darzustellen dann eignet sich $\mathrm{Ge}$ walt und Kriminalität (.) sehr gut (.) ähm. (.) Eine weitere These ist dass (.) viele Formen von Gewalt tabuisiert werden (.) oder (1.5) also ich denke wenn ich jetzt zum Beispiel an Gewalt im Verkehr denke (.) bin ich nicht sicher ob es (.) the tabuisiert wird (.) aus Schamgefühlen glaub ich eben nicht wie zum Beispiel äh (.) Kindesmisshandlungen und Gewalt von Männer gegen Frauen häusliche Gewalt des wird wird tabuisiert glaub ich als Schamgefühlen (.) weil man nicht genau hinschauen will weil beim Gewalt im Verkehr ist es zum Beispiel so (.) glaube ich hat es nichts mit Schamgefühle zu tun sondern einfach mit äh das Recht des Stärkeren dass sich dass sich eigentlich bei den Menschen ähm (2.0) ich muss vielleicht wie ausholen für mich ist wie äh die Menschen sind weniger sozial eingestellt (.) mehr auf sich selber bezogen und das zeigt sich auch in den Formen von Gewalt im Verkehr zum Beispiel oder Aggressivität sagen wir mal Aggression im im im im Verkehr (.) ähm also diese Themen eignen sich aus verschiedenen Gründen nicht sie zu thematisieren weil man dann in die Tiefen gehen Tiefe gehen müsste. Die Jugendgewalt eignet sich perfekt weil sie plakativ ist und weil sich die Jugendliche gar nicht wehren können weil sie das gar noch nicht gelernt haben. (.) Oft ost äh auch äh Jugendliche sind Jugendlich dankbar (.) weil Jugendliche müssen sich auflehnen gegen die Gesellschaft gegen die Erwachsenenwelt gegen das Elternhaus (.) das ghört gehört medizinisch ähm von den von von den (.) Hormonen gehört das dazu $\uparrow$. (.) Sie machen das auch $\uparrow$ (.) und es ist ja so dass sie ja das lernen müssen und sie können das noch nicht sie können sich noch äh differenziert ähm artikulieren (.) ähm (.) äh und dann sind sie natürlich prädestiniert dazu (.) um um das plakativ äh so so so anzukreiden (.) ohne dass man sich eigentlich wirklich damit befassen muss. (.) Das ist so (.) der negative Teil (.) ich denke es gibt auch einen positiven und da mein (.) meine ich dass die (...)" (Zeilen 41-60)

Herr Tanner stützt seine gesellschaftskritischen Argumentationen durch drei Thesen: Erstens sieht er im Kontext von Gewalt und Kriminalität eine gesellschaftliche Nachfrage an der Diskriminierung bestimmter Bevölkerungsgruppen (z.B. »Migrantinnengruppen«). Zweitens beobachtet er eine Tabuisierung anderer »Gewaltformen« (beispielsweise im Straßenverkehr, häuslicher Gewalt, von Männern ausgeübte Gewalt an Kindern und Frauen), was er an »Scham« und Veränderungen bezüglich moralisch-ethischer Einstellungen festmacht: Menschen seien immer weniger sozial eingestellt und mehr und mehr auf sich bezogen. Gesellschaft sei seiner Beobachtung nach nicht dazu bereit, dies kritisch zu reflektieren. Drittens nimmt er an, dass Jugendliche im Gegensatz zu anderen Gruppen »leichte Opfer« von Diskriminierungsprozessen seien. Aufgrund biologischer Ursachen bedienten Jugendliche bestimmte 
Handlungsmuster, aufgrund derer sie ins Visier von Diskriminierungsprozessen gerieten (etwa der Drang, sich gegen die Gesellschaft, gegen die Erwachsenenwelt und gegen das Elternhaus aufzulehnen). Sie könnten sich noch nicht »differenziert artikulieren«, um sich gegen Diskriminierung zur Wehr zu setzen. In seiner Argumentation entwickelt Herr Tanner eine Defizitperspektive auf Jugendliche, die er biologisch begründet. Sowohl das Auflehnungsbedürfnis Jugendlicher als auch deren mangelnde Artikulationsfähigkeit gehörten medizinisch, von den Hormonen her, dazu und machten Jugendliche zu beliebten »Opfern« gesellschaftlicher Diskriminierung. Diese Argumentation impliziert außerdem eine Differenzierung zwischen der »Erwachsenengesellschaft« und Jugendlichen. In dieser Argumentation verknüpft Herr Tanner seine gesellschaftskritische Positionierung mit seiner Positionierung als (leitender) Jugendarbeiter: Er bezieht seine Gesellschaftskritik auf Gesellschaft, gedacht als von Erwachsenen dominierte Welt, vor der er Jugendliche in Schutz nimmt. Seine gesellschaftskritische Positionierung geht also mit einer Defizitperspektive auf Jugendliche einher, die ihm wiederum ermöglicht, Jugendliche in Schutz zu nehmen. Insofern er offene Jugendarbeit als Schutzraum denkt, verknüpft er seine gesellschaftskritische Perspektive also mit seiner Position als (leitender) Jugendarbeiter einer Einrichtung offener Jugendarbeit. Neben den »negativen« Aspekten der Thematisierung von Jugendgewalt sieht Herr Tanner auch »positive«:

Herr Tanner: "(...) einen positiven und da mein (.) meine ich dass die Stadt ,Name der Stadtı sehr gute Arbeit leistet äh. Ich kann das nicht vergleich mit anderen Städten aber ich bin der Meinung dass 'Name der Stadt hier hervorragende Arbeit leistet durch das dass dass dass sich die ges dass man sich mit äh dass dass Jugendgewalt ein Thema ist gibt es tatsächlich (.) äh Leute (.) die sich sehr intensiv damit befassen. (.) Also es gibt eine Gewaltpräventionsstelle die (.) sehr Gute arbeit leistet. (.) Die mit innovativen Projekte äh Projekten unterwegs ist die die ich wirklich sehr gut finde. Ich denke auch die Geschichte nach den äh Jugendunruhen also nein äh äh nach den zwei äh Ausschreitungen die äh die es letztes Jahr gab (.) äh (.) die (1.5) den Kontakt die die Verwaltung (.) mit den Jugendlichen gesucht hat über Facebook das kann man ein bisschen belächeln wenn man will (.) aber (.) es war es ist eine Stadtverwaltung $\uparrow$ (.) die (.) den Kontakt zu den Jugendlichen haben (.) und aufgenommen in einen Dialog Maßnahmen abgeleitet haben. ((Räuspert sich)). Das Bewilligungsverfahren jetzt das sie das sie gebracht haben (.) auch das könnte man belächeln. (.) Ich find es super (.) dass eine Verwaltung (.) auf diese Art auf Jugendliche hingeht und innen Möglichkeiten gibt und in der Praxis müss muss sich das zeigen vielleicht funktionierts überhaupt nicht vielleicht ist es gar nicht das was es braucht aber es ist ein Schritt (.) zu den Jugendlichen hin und wo die Jugendlichen die Möglichkeit haben also einige zumindestens (.) sich zu artikulieren (.) in Kontakt zu Verwaltung () und ich denke das ist der pos der positive Aspekt der der 
ganzen Geschichte also ich möchte jetzt hier auf keinen Fall nur negativ sehen." (Zeilen 61-73)

Aus dem Jugendgewaltdiskurs, zu dem sich Herr Tanner zunächst kritisch positioniert, resultieren aus seiner Perspektive auch positive Entwicklungen, z.B. die Jugendarbeit der Stadt, in der sich auch seine Einrichtung befindet. Sein Lob der städtischen Projekte bindet er an Begebenheiten in jüngster Zeit. »Jugendunruhen« bzw. »Ausschreitungen« im öffentlichen Raum hätten die Stadt dazu veranlasst, mittels moderner Kommunikation mit den Jugendlichen in »Dialog « zu treten, um deren Anliegen herauszufinden. Dabei sei ein Verfahren entstanden, das den Jugendlichen die Möglichkeit bietet, öffentliche Veranstaltungen anzumelden. Herr Tanner bewertet es positiv, dass die Jugendlichen über dieses Verfahren die Möglichkeit haben, sich zu »artikulieren«. Rechtlich bewilligte Artikulationsformen sind aus seiner Perspektive die angemessene und ernst zu nehmende Möglichkeit der Artikulation Jugendlicher. Herr Tanner beschreibt hier einen Institutionalisierungsprozess, indem er gute städtische Präventionsarbeit mit Institutionalisierung und Formalisierung von Kommunikation verbindet. Dem biologisch begründeten Mangel an Artikulationsfähigkeit setzt Herr Tanner somit institutionalisierte Möglichkeiten der Artikulation entgegen. Dadurch wird den Jugendlichen nicht nur ermöglicht, sich zu artikulieren, sondern sie werden eigentlich auch gezwungen, dies zu tun. Eigene, selbst organisierte oder initiierte Wege der Kommunikation, die Herr Tanner als »Jugendunruhen« oder »Ausschreitungen « bezeichnet, seien als Artikulation nicht ernst zu nehmen. Die Interviewerin bittet ihn darum, diese Begebenheiten nochmals zu explizieren.

Interviewerin: "Hmhm und können Sie das nochmal beschreiben also dass äh war mir gar nicht so klar (.) dass da dieser Kontakt äh stattgefunden hat wie des (.) wie des zustandegekommen ist diese (1.5) diese Geschichte mit dem Kontakt über Facebook was ist da genau passiert?"

Herr Tanner: "Ja also da da ging es die beiden Ausschreitungen äh am (.) äh ok nein es war im im August (.) August Semptember letztes Jahr äh es ging um eigentlich um die illegalen Partys (.) und (.) die Art und Weise wie die Polizei diese auflöst (.) und dass die Jugendlichen reklamiert haben es gibt viel kommerzielle Angebote die wir entweder uns nicht leisten können (.) oder das nicht wollen. (.) Wir wollen selber organisieren und wir haben keine Räume (.) und dann ähm ist (.) das hab ich alles nicht mitbekommen falls Sie das falls Sie inhaltlich mehr wissen wollen müssen sie sich bei ,Vorname und Name einer Fachperson‘."

Interviewerin: "Den hab ich noch offen also mit dem hab ich einen Termin." Herr Tanner: "Also dann erzähl ich Ihnen gar nicht so viel weil er weiß es nämlich äh viel genauer. (.) Sie haben auf Facebook einen einen einen äh (.) einen Wiki oder wie auch immer dass sie das was sie da gestartet haben (.) wo (.) die Jugendliche rückmelden 
konnten was sie eigentlich wollen und was sie brauchen (.) und daraus hat sich (.) das kann Ihnen ,Name der Fachperson، sich auch geben äh. Das war vor etwa einem Monat eineinhalb in der Presse äh hat es ein äh vereinfachtes Bewilligungsverfahren (.) für Veranstaltungen für Jugendliche bis 25 Jahren äh hat die Stadt eingeführt und da haben die Jugendlichen Möglichkeit und und junge Erwachsene haben die Möglichkeit bis irgendwie sieben Tage vorher ein Bewilligungsverfahren einzureichen und dieses auch zu erhalten. Es hat es hat natürlich heikle Punkte äh wie zum Beispiel dass die jung die Organisatoren dann verantwortlich sind und was heißt dann das (.) wenn es dann wirklich was gibt (.) äh aber aber eben die Jugendlichen haben jetzt die Möglichkeit ihre Partys zu legalisieren (.) und da hat es wie (.) verschiedene Reaktionen gegeben. Es hat Reaktionen gegeben von Jugendlich also jetzt von Jugendlichen die finden ähm super mach ich (.) Möglichkeit etwas Möglichkeit etwas zu machen und andere gefunden haben spinnt Ihr (.) für uns ist wichtig dass wir illegal sind wir wollen es gar nicht legalisieren. (.) So aber es entsteht ein Dialog es ist ein Dialog entstanden und und die die jetzt illegal sein wollen müssen wie ein anderes Argumentarium bringen. (.) Sie können nicht mehr sagen (.) man kann nicht (.) sondern man kann aber es passt uns nicht oder wie auch immer so. (1.5) Ich denke da kann Ihnen Herr ,Name der Fachperson، dann auch zu den Resultaten oder zu zum Dialog der effektiv entstanden ist auf Facebook da kann er Ihnen etwas sagen da weiß ich nichts." (Zeilen 74-95)

Herr Tanner beschreibt das Vorgehen der Polizei, das die »Auflösung« von »Ausschreitungen« bzw. »illegalen Partys« zum Ziel gehabt hat. Dabei nimmt er auch die Perspektive der Jugendlichen ein, die damit ein Interesse daran zu Ausdruck gebracht hätten, Veranstaltungen nicht-kommerzieller Art selbst zu organisieren und Räume dafür zu benötigen. Er schildert, wie die Stadt über das Internet Kontakt mit den Jugendlichen aufgenommen hat, die auf diese Weise ihre Bedürfnisse kommunizieren konnten. Daraus sei das Bewilligungsverfahren entstanden, im Rahmen dessen Jugendliche ihre Veranstaltungen eine Woche vorher anmelden können. Seinen Schilderungen sind Bewertungen zu entnehmen, die eine klare Positionierung zu Stadt bzw. Polizei erkennen lassen: Herr Tanner bezeichnet die Veranstaltungen der Jugendlichen von vornherein als »illegale« »Ausschreitungen« und relativiert diese Bezeichnungsweise nicht. Die Kontaktaufnahme durch Stadt und Polizei bezeichnet er hingegen als »Dialog«, der den Jugendlichen Möglichkeiten eröffne und Pflichten mit sich bringen würde: Sie hätten die Möglichkeit, ihre »Partys zu legalisieren«, was jedoch eine gewisse Verantwortung erfordere. Wie bereits erwähnt, haben Jugendliche nun auch den Zwang, sich zu positionieren: Die Entscheidung gegen das Bewilligungsverfahren sei immer eine bewusste Entscheidung für Illegalität. Herr Tanner stellt auf Nachfrage der Interviewerin also zunächst die Perspektiven von Polizei und Jugendlichen einander gegenüber. Im Interviewverlauf argumentiert Herr Tanner jedoch zunehmend für Stadt und Polizei, was er auch mit der defizitorientierten Pers- 
pektive auf Jugendliche begründet, deren Handlungsentscheidungen und Begründungsmuster er in die Sphäre der Illegalität verortet. Herr Tanner zweifelt daran, dass alle Jugendlichen die erforderliche Verantwortung dafür aufbringen können, und bewertet somit den Rechtfertigungsdruck Jugendlicher für die Entscheidung, auf das Bewilligungsverfahren zu verzichten, als Erfolg der Maßnahme. In seinen Bewertungen vertritt Herr Tanner weniger die Sichtweisen der Jugendlichen, sondern vielmehr die der Stadt, der Polizei und der öffentlichen Medien, vor deren Hintergrund er die Aktivitäten, Forderungen sowie Begründungen der Jugendlichen disqualifiziert. Letztlich beschreibt er hier einen Prozess der Institutionalisierung öffentlichen Raums, deren In-diePflicht-Nehmen der Jugendlichen er als Erfolg städtischer Präventionsarbeit ansieht. Herr Tanner empfiehlt einen anderen Gesprächspartner, der über die »Resultate« und den »Dialog, der effektiv entstanden ist«, Auskünfte geben kann. Der Verweis auf die Bedeutung von Resultaten und Effektivität impliziert einerseits eine Orientierung an einer Richtigkeit der Deutung des Geschehens seitens der Stadt und der Polizei und geht konform mit einer defizitorientierten Perspektive auf Jugendliche, die Herr Tanner v.a. mit deren mangelnder Artikulationsfähigkeit begründet. Andererseits impliziert dieser Verweis eine Orientierung an kriminalpolitischer Programmatik, die auf Wirksamkeit, Effektivität und Erfolge von Maßnahmen abzielt. Herr Tanner schildert die Begebenheit, wie bereits festgehalten, als Beispiel einer positiven Entwicklung, die sich aus dem Jugendgewaltdiskurs ergeben habe, womit er sich von seiner gesellschaftskritischen Positionierung distanziert. Diese positive Entwicklung bezieht Herr Tanner jedoch primär auf Aspekte des Nutzens aus der Perspektive von Stadt und Polizei: Der Erfolg bemesse sich also weniger daran, dass Jugendliche das Bewilligungsverfahren nutzen würden, sondern vielmehr daran, dass diejenigen Jugendlichen, die davon Abstand nehmen, unter erhöhten Rechtfertigungsdruck geraten würden. Aus dieser Sequenz gehen zwei zentrale Aspekte hervor. Erstens die Herausforderung, unterschiedliche Positionierungen zu vereinbaren: eine gesellschaftskritische Haltung gegenüber dem Jugendgewaltdiskurs und eine an den Interessen der Stadt, der Polizei und der Öffentlichkeit ansetzende Jugendarbeit, die sich vom Jugendgewaltdiskurs nährt. Damit positioniert sich Herr Tanner auch zwischen zwei Interessengruppen, die der Jugendlichen und die der »Erwachsenengesellschaft«. Zweitens entwickelt Herr Tanner eine defizitorientierte Perspektive auf Jugendliche, die zur Vereinbarkeit der beiden unterschiedlichen Positionierungen beiträgt. Diese Ambivalenzen spiegeln sich in Herrn Tanners Beschreibungen »guter « Jugendarbeit, die von einer Spannung zwischen Intervention und »Wiedergutmachen « geprägt ist. Die Oszillation zwischen verschiedenen Sprecherrollen lässt sich auch im Interview mit Herrn Gold beobachten. An der Eingangssequenz zeigt sich, dass auch er sich, ähnlich wie Herr Tanner, von der Adressierung als Experte für Jugendgewaltprävention zugunsten einer ge- 
sellschaftskritischen Perspektive zunächst distanziert. Dabei problematisiert Herr Gold Jugendgewalt explizit als semantisches Problem, das er gleichzeitig auch normalisiert. Wie entwickelt Herr Gold vor diesem Hintergrund seine Expertise in Bezug auf seine Organisation? Zunächst greift die Interviewerin Herrn Golds Beobachtung auf, dass Jugendgewalt so stark thematisiert wird:

Interviewerin: "Hmhm (.) hmhm (.) und Sie sagen, dass es eben sozusagen ab und zu eben so gehypt wird? Also ich sag dis jetzt mal in meiner Sprache. (.) Ähm (.) können Sie sagen ähm gibt es da eine Entwicklung jetzt die Sie grad sehen in den letzten zehn Jahren oder zwanzig Jahren (.) wie wie schätzen Sie da eine Entwicklung ein? (.) In der öffen- ja in der Schweiz was ist oder woran könnte es liegen das es-“

Herr Gold: "Ja (.) ähm also äh ich denke man kann verschiedene Sache uusmache das einte ist sicher (.) dass es zum Teil s äh s Medienthema isch ähm und zum Teil des Summerlochmedienthema sehr allgemein es muss nicht nur im Sommer wenn halt grad ned da so waahnsinnig viel äh Spannendes berichtet (.) nimma die Gschichte un tu sie halt in der in der Masse mit ( ) und (.) und äh und breitträtä. (.) Ds anderen ist sicher dass es ja (.) Erhebige gäh hät wo ganz klar auzeigt ist dass die Schwiz $\uparrow$ i räd wirklich jetzt von Schwiz (.) dass es äh dass dass dä (.) a Azeige zuegno häd u wia d Verurteilungen zuegno häd i dem Bereich. (.) Ob das jetzt natürlich a Zuanahm von der effektiven Jugendgwalt isch wiss mer äh ch ma in dem Sinn nöd gnau belege. (.) Es hät zumindest sicher $n$ Sensibilisirig ghä (.) in der Gsellschaft dass des wirklich so ist und also generell da dranknüpfä genau die Sensibilisirig hätt sicher au stärker stattgfundä in de nünzger Johr und i der i der erschte zei Johr etz vo dem Jahrhundert. (.) Ähm (.) wo äh (.) wo ma einfach wirklich denn (.) quasi bi jedem chline Vorfall scho ufschreit und find a ds jetzt Gwalt wo ma früher no gsait häd boa (.) Jungs (.) bluetigs äh bluetige Nase und des brauchts au (.) das ghört irgendwia dazua und hüt isch des schon sofort en (.) Fall für Mobbing und es werded alle Experten einzoge und (.) psychologisiert und so witer und (schnauft). Das machts dann zum Problem wo denn gern breitträtä wird und diskutiert wird also die Entwicklig gsehn ich (.) obs wirklich a Entwicklig vo der Zuanahm von de Jugendgwalt ghä häd ((schnauft)) chan i jötzt nöd (.) abschließend beurteile." (Zeilen 20-38)

Herr Gold stellt zwei Beobachtungen zur gesellschaftlichen Thematisierung und Problematisierung von Jugendgewalt an, wobei er diese gleichzeitig zu begründen und zu bewerten sucht. Erstens beobachtet er, dass Jugendgewalt ein beliebtes Medienthema ist, insbesondere wenn es gerade an interessanten Themen mangelt. Dies impliziert die Beschreibung einer Öffentlichkeit, die diesem Thema etwas abgewinnen kann. Zweitens beobachtet er eine zunehmende Sensibilisierung bezüglich der Jugendgewalt, was er an der polizeistatistischen Erfassung derselben festmacht, wobei auch hier eine Orientierung an »Effektivität« sichtbar wird: Eine erhöhte Rate der Jugendgewalt erklärt er hier implizit über eine erhöhte Anzeigebereitschaft. Seine Empörung über eine übertriebene Sensibilität verdeutlicht er an einem Beispiel: Seiner Beob- 
achtung nach »gehörte« früher eine »blutige Nase [...] irgendwie dazu«. Heute werde dieser jedoch eine so hohe Aufmerksamkeit zuteil, dass »alle Experten« einbezogen würden, die »psychologisieren«. Herr Gold schildert damit einen Prozess der Pathologisierung von Jugendgewalt, der insbesondere durch die Psychologie angetrieben wird, womit er sich von dieser Expertengruppe distanziert. Er hingegen sieht bestimmte Formen von Jugendgewalt als »normal« an, insofern sie irgendwie dazugehören. Damit versucht er, übertriebene Formen der »Pathologisierung « zu entschärfen. Die Interviewerin fordert Herrn Gold dazu auf, weiter über dieses Thema zu sprechen und dabei den Zusammenhang zwischen Jugend und Gewalt zu berücksichtigen.

Interviewerin: "Hmhm (.) danke (.) und ähm (.) wie (.) wie ist des eigentlich (1.5) also wenn wir ja nochmal bei diesem Thema bleiben also warum (.) geht es heute um Jugend also um Jugend und Gewalt (.) wie hängen (.) wie hängt des zusammen?»

Herr Gold: "((schnauft))"

Interviewerin: "Es (.) ja (2.5) wenn man so an die Formen von Gewalt denkt dies vielleicht noch gibt."

Herr Gold: "((lacht laut)) Ja (.) isch a so ja genau ebbe. (.) Die Frog stellt sich ganz klar oder $\uparrow$ das isch so äh äh ja wieso muss es zammhan also (.) i mein in der Jugend mit mit mit de mit de (.) e entwickligspsychologische ähm (.) Prozess wo passieret isches eben ( ) quasi individuelreschpektiv na() isches eben richtige (.) Phasa und sehr en äh (.) en en heiße Phase und Jugendliche (.) äh probieret Grenzen us und sie überschriitet Grenz und das wird immer sehr heftig wahrgno von de Erwachsene her oder $\uparrow$. Aber $\uparrow$ es isch so es isch ( ) schinheilig wenn die Erwachsene halt eifach sehr viel auf anderen Ebenen eigentlich Gwalttätigkeit passieret oder Umverteilig von Wohlstand wo au no a ganz ein gwüsse Teil (.) zum gwüsse Spurvon Gwalt innehät ähm ähm wird dann eifach Jugend wo s dann halt wirklich noch am heftig wäre und in Schlägerei uusarte det wird denn gern mit dem Finger drufzeigt und die Erwachsene saget jaa und des is ganz schlimm und so so witer wähend wir eigentlich (.) die Erwachsenen halt am Arbeitsort gibts Mobbing und äh und Lüt wo sich mün krankschriebe loa ebbe gnau aus dene Gründ bispielswis (.) ähm wo wo eigentlich gnau in die glich (.) Richtig gönd oder $\uparrow$. (.) Und ich glaub natürlich was auch immer no $n$ Punkt ist wir hän natürlich immer die Hoffnung so Jugend wo s a die Zuakunft da darstellt von der eigenen Gsellschaft (.) ähm dass die ja dann au so ein bsonders bsonders guad und und liab und schön si und sobald einmal wieder negative Nachricht chommen denn is des fascht ( ) Igschtändnis für die Erwachsenen selber oder $\uparrow$. Aha ok a nei die sin ja gar ned a so und was passiert denn und äh wir sind ja irgendwie auch dafir verantwortlich und wieso ist das so a. (2.5) Gern nachfrage wenn ich mich irgendwie unklar aus usdrücke oder so hä $\uparrow$.“ Interviewerin: "Ja, nee, ich ich komm ganz gut mit." Herr Gold: "((Lacht laut)) Is guad ((lacht laut))“ (Zeilen 39-57) 
Im Gegensatz zur vorhergehenden Sequenz, in der sich Herr Gold von der Pathologisierung von Jugendgewalt, die von Psychologen angetrieben wird, distanziert, nimmt er hier selbst eine entwicklungspsychologische Perspektive auf Jugendliche ein. Obwohl er an seiner kritischen Haltung gegenüber der gesellschaftlichen Thematisierung von Jugendgewalt festhält, indem er den Zusammenhang zwischen Jugend und Gewalt infrage stellt, beschreibt er die Jugendphase als »heiße Phase«, in der Jugendliche »Grenzen ausprobieren« und »überschreiten«. Damit normalisiert er Jugendgewalt als jugendliches Handeln in Abgrenzung zu deren Pathologisierung. Dabei verallgemeinert er entwicklungspsychologische Prozesse und stellt den Jugendlichen die Erwachsenen gegenüber. Herr Gold setzt sich also in ein ähnliches ambivalentes Verhältnis zwischen Jugendlichen und Erwachsenen wie Herr Tanner. Er kritisiert die Erwachsenen dahingehend, dass sie Jugendgewalt skandalisieren, andere Formen von Gewalt wie sozialstrukturelle Ungleichheiten und Mobbing am Arbeitsplatz hingegen nicht, was er als »scheinheilig« bezeichnet. Er rechnet sich selbst zu der Gruppe der Erwachsenen und vollzieht deren Sorge nach, die sie hinsichtlich der Verantwortung für die »eigene Gesellschaft« hat. Das Selbstverständnis, in den Jugendlichen eine Hoffnung zu sehen, werde durch »negative Nachrichten« erschüttert. In dieser Sequenz handelt Herr Gold ähnlich wie Herr Tanner unterschiedliche Sprecherpositionen aus. Einerseits zeigt er Verständnis für jugendliches Gewalthandeln und kritisiert dessen Dramatisierung und Skandalisierung. Andererseits zeigt er auch Verständnis für die Sorge Erwachsener in Bezug auf die Zukunft der Gesellschaft.

Interviewerin: "Ja glauben Sie dann dass dann (.) ja (.) aus der Perspektive der Jugendlichen bestimmte (.) ja Formen von Gewalt dann legitim sind $\uparrow$ die dann die Erwachsenen (.) äh problematisieren oder gibt es das (.) legitime Gewalt?" Herr Gold: "((lacht)) Guate isch ne guate Frog ja ich glaubs i cho s jetzt nög sage für alle Jugendlich is dia Form wo legitim isch. (.) Mol aber ich glaub scho (.) und was mir au nöd döff vergessse isch es (.) das eds grad (.) äh (.) ich erleb das sehr in miner $n$ Arbeit mit Schualchlasse (.) wo ich jedes Semester irgendwie mehrer (.) duzende bis sogar hunderte Chinder und Jugendliche han won ich so (.) prozessorientierte Projekt begleit unterricht und animiere äh wo wo halt i dene Stufe Schualstufe o ich denn das mache das sind meischtens solche die (.) Sekstufe Realstufe ich weiß ned inweifern was Du Schwizer Schualsystem schon chennst $\uparrow$ aber (.) das sind dann alt schon immer die Schualstuf wo ma a jetzt merkt da fängt demographisch aha $\uparrow$ (.) da hätts eifach a weng megaviel Chind u Jugendliche die wo uus andere Chulturkreis kömmet oder zumindest eine Eltere Teil usm Chulturkreis kömmet wo (.) wo südländisch is sag mas mal so. Und det isch zum Beispiel halt s Thema Eehr und Ruef eifach viel wichtiger und denn isch denn aber für die Jugendliche legitim sich z wehre au gwalttätig sich z wehre wenn mal öbber sait di Muetter is ne Schlampe oder was a immer (.) wo als Schwizer jetzt früher ganz ehrlich gsait (.) eifach chalt glo häd oder wie das auch hoiß ja (.) denn halt ähm 
ich weiß ja was mini Muetter isch (.) is mir glich (.) und (.) dann nehmets natürlich das (.) wirklich als sich wehre und nöd als Gwalt denn sofort dri schlo und hey da muess ich mich verteidige (.) und durum hanich (.) erläb i das scho dass (.) deilwis Gwaltsachen au als legitim von Jugendliche ganz klar agseh werdet sich z wehre. (.) Es isch innen aber sehr sehr bewusst (.) ähm dass es au Gwalt isch. (.) Also es isch nöd so dass die Jugendlichen umananlaufed drinschloged oder auf Facebook sich gegesitig fertigmache und sich überhaupt nicht bewusst sind was des isch sondern des Bewusstsi is schon da bei de Jugendliche dass die Erfahrig mach ich scho ja (.) von dene Tätigkeite ähm was das für nachhaltige Wirkunge häd und so wiiter (.) das is mir natürlich dann teilwis nöd bewusst wil wil in dem Alter sie sich eifach (.) ja äh die Gedanke no nit chön mache aber (.) was es bedütet (.) das is innen sehr bewusst oder $\uparrow$ ". (Zeilen 58-77)

Auf die Frage der Interviewerin, ob es aus der Perspektive Jugendlicher legitime Gewaltformen gebe, die Erwachsene illegitim finden, und ob es überhaupt legitime Gewalt gebe, eröffnet Herr Gold eine erfahrungsbezogene Perspektive in Bezug auf seinen Fachbereich: In der Schule werde jugendliches Gewalthandeln nicht als entwicklungspsychologisches Muster relevant, sondern als »kulturelles«. »Kulturelles« Gewalthandeln sei aus der Perspektive bestimmter Jugendlicher legitim. In dieser Hinsicht zieht Herr Gold eine Differenz innerhalb der Gruppe der Jugendlichen, mit denen er im Rahmen seiner Projektarbeit an Schulen arbeitet. Für »Kinder und Jugendliche, die aus einem südländischen Kulturkreis kommen«, seien die Themen Ehre und Ruf sehr wichtig, sie fühlten sich schneller angegriffen und würden schneller eine Verteidigungshaltung einnehmen als Schweizer Kinder und Jugendliche. »Sich wehren, auch sich gewalttätig zu wehren«, stellt sich nach Herrn Golds Erfahrung als legitime Gewaltform aus der Perspektive »südländischer« Kinder und Jugendlicher dar, die von anderen Jugendlichen und auch ihm selbst als illegitim angesehen wird. Gewalthandeln zeige sich beispielsweise als »reinschlagen«. Den Jugendlichen, so Herr Gold, sei bewusst, dass es sich dabei um Gewalt handelt, sie hielten dies jedoch trotzdem für legitim und seien sich nicht der »nachhaltigen Wirkungen« bewusst. Ähnlich wie Herr Tanner entwickelt Herr Gold hier eine entwicklungsbedingte defizitorientierte Perspektive auf Jugendliche, die sich jedoch primär auf Jugendliche bezieht, die aus anderen »Kulturkreisen« kommen, z.B. »südländische« Jugendliche. Im Gegensatz zu Erwachsenen, Fachpersonen oder Schweizer Jugendlichen könnten sie die langfristigen Konsequenzen ihres Handelns nicht einschätzen. Zwei relevante Aspekte werden an dieser Stelle festgehalten: Erstens positioniert Herrn Gold sich, ähnlich wie Herrn Tanner, zwischen den Interessen der Jugendlichen und der Erwachsenen, wobei er zudem eine »kulturelle« Differenzlinie markiert. Die Normalisierung von Jugendgewalt erfolgt also über Kulturalisierung von Jugendgewalt. Zweitens fällt in diesem Zusammenhang eine ähnliche defizitorientierte Perspektive auf Jugendliche auf. Dieser 
Ähnlichkeit der Orientierungsmuster wird trotz unterschiedlicher institutioneller bzw. organisatorischer Kontexte im Folgenden Rechnung getragen und sie wird vertieft. »Normalisierung « und »Kulturalisierung « von Jugendgewalt werden als relevante Aspekte weiterverfolgt. So greift die Interviewerin diese Thematik auf, nachdem Herr Tanner seine Erzählung über das Bewilligungsverfahren beendet hat.

Interviewerin: "Ok (.) spannend (.) gut und Sie haben äh vorhin ähm (.) darüber gesprochen dass es ja auch verschiedene Formen von Gewalt äh gibt die einen werden eben stärker thematisiert. (.) Können Sie da nochmal sagen welche Formen von Gewalt gibt es Ihrer Meinung nach und mit welchen Formen haben Sie in inrer Arbeit zu tun oder hat die 'Name der Einrichtung‘ zu tun?"

Herr Tanner: „Hmhm (2.0) dann dann äh (.) ja eben da müsste man eben noch Gewalt definieren aber das lassen wir jetzt mal oder $\uparrow$ ((lacht))."

Interviewerin: "Sie können natürlich wenn sie möchten ((lacht)). Ich weiß nicht ob Sie (.) ob Sie das tun ((lacht))"

Herr Tanner: "Äh (.) ja nein das ist das ist äh das find ich no guter Input das wäre mal etwas das wir äh (.) dass wir eigentlich- ((es klingelt an der Tür)) definieren könnten darf darf ich schnell?"

Interviewerin: „Des macht nichts ja des is schon ok. ((lacht))"

((Herr Tanner geht aus dem Büro zur Haustür, öffnet und spricht mit einer Person. Er kommt nach knapp zwei Minuten wieder zurück und setzt sich wieder.))

Herr Tanner: "Also dann versuch ich mal Gewalt zu definieren äh ich definier mal Macht. (.) Ich weiß es ist nur eine Definition aber sagen wir Macht ist die Fähigkeit ähm (1.5) die Realität eines Anderen zu bestimmen. (.) Und Gewalt (.) ist dies mit äh (.) mit äh Druck oder eben mit mit mit mit rep mit Gewaltmaßnahmen ob psychisch oder körperlich durchzusetzen so. (.) Also (.) äh mit der einen Form mit der wir äh konfrontiert werden (.) oder äh ja die Jugend dann konfrontiert wird ist die tatsächliche Gewalt (.) zwischen Jugendlichen oder von Jugendlichen gegenüber anderen ähm Altersgruppen aber die Gewalt von Jugendlichen äh und da ist die Gradwanderung zwischen zwischen Aggression (.) und Gewalt ist fließend und nicht immer äh klar trennbar äh insbesondere bei den Interventionen. (.) Äh s nnnnnnnnnnnn da ist jeweils schwierig zu unterscheiden es ist auch also und und es geht nicht um eine theoretische Unterscheidung es geht darum (.) wenn Vorfälle sind in einem Treff oder in einer Gruppe oder an einer Veranstaltung (.) wo interveniert man (.) und wo lässt man es eben noch als normale Aggression (.) laufen wo man das Gfühl hat die Jugendlichen können und müssen das untereinander äh händeln und da (.) s hängt von den Jugendlichen ab (1.5) also die die in dieser Situation da sind wie gut kennen sie sich ähm haben sie schon solche Situationen schon gehabt sind es Jugendliche die sich nicht kennen die wir nicht kennen es hängt aber auch von den Mitarbeiterinnen ab wo ist meine Schwelle wo hab ich noch Sicherheit etwas laufen zu lassen (.) ähm (.) was ertrage ich mehr oder weniger (.) also mit diesem Thema sind wir äh sind wir äh (.) äh konfrontiert (.) äh (.) und zwar sowohl in der Arbeit selber also 
in den Treffs oder in den äh bei den Veranstaltungen. (1.0) Dann natürlich aber auch äh die Gewalt die außerhalb passiert (.) also von unseren ju also unseren (.) Jugendlichen die unsere Einrichtung besuchen gegenüber anderen Jugendlichen aber auch von anderen Jugendlichen gegenüber Jugendlichen die dann zu uns kommen. (1.0) Dann gibt es äh die ähm häusliche Gewalt (.) äh die Jugendliche erleiden zu Hause und und zu uns kommen (.) äh und die ist das ist eben das Tabuisierte das ist das kommen wir sehr sehr wenig äh dran i oder oder ran an diese Themen ich höre ganz wenig. (.) Das ist für mich eigentlich ein Zeichen das wäre spannend das müsste ich mit meinen Leuten besprechen kommt sie gar nicht vor (.) also oder sie kommen schon vor aber- kommt kommen die Themen nicht an uns (.) heran (1.5) oder kommen sie einfach nicht zu mir (1.0) weil eigentlich sind es oft s s sind es Themen die sehr schnelle eine Intervention bräuchten und die Leute müssten sich dann bei mit abstützen was wir was wir dann was wir dann in der in der Situation machen (.) aber das ist denk ich sicher eine Form von Gewalt mit der wir äh (.) mit der wir konfrontiert sind (.) ohne sie auf dem Tisch ausgesprochen zu haben. Und dann gibt es so die die gesellschaftliche äh äh Gewalt ähm (1.5) zum zum Beispiel gegenüber Migranten Jugendlichen (.) die äh dann also wir hatten eine politische Veranstaltung letztes jahr im Oktober und die ist ziemlich eskaliert. (.) Wir hatten Journalisten vom 'Name einer Zeitung، vom 'Name einer anderen Zeitung، die haben das (.) die habe einen wunderbaren Bericht geschrieben ((lacht)) gar nichts von der geladenen Stimmung. (.) Es war es war eigentlich erstaunlich (.) aber aber schlussendlich ists eskaliert man hat gemerkt wie die Jugendlichen (.) wirklich aggressiv waren (.) vor allem gegen den SVP Politiker (.) und die Journalistin war dann nicht mehr da als nach der Veranstaltung es zu ei zu einem Geschupfe kam als dann der geflüchtet ist und ich dem hintennach (.) so (.) und (.) jetzt kann man sagen ok die Jugendlichen haben wieder mal bewiesen dass sies nicht können vor allem die exjugoslawischen haben bewiesen dass wir schon Recht haben dass die nicht zu uns gehören (.) oder man kann sagen (1.0) dass Aggressionspotential dass an dieser Veranstaltung kam zu aller erst mal sehr stark verbal (.) ähm. (2.0) Das ist der Frust den sie tragen immer zu hören ihr ihr seid die Raser ihr seid die Kriminellen ihr seid die Gewalttätigen und so (.) ich denke das ist eine Form von von Gewalt die wir zu spüren bekommen (.) oder mit der wir äh zu zu arbeiten haben (.) ich denke auch auf dem Arbeitsmarkt (.) halt die Benachteiligung ist (.) je nach dem was für eine Sprache was man hat (.) aber auch schon dass diese ähm (.) also dass Jugendliche mit schlechterer Schulausbildung viel schlechtere Chancen haben auf dem (.) auf dem Arbeitsmarkt (.) ähm. (.) Ja es ist ( ) zu sagen ob das jetzt eine Form von Gewalt (.) ist (.) aber es ist sicher eine Form von Macht $\uparrow$ (.) die wirkt und (.) und da hab ich einen ganz wichtigen äh wichtigen Teil für mich den ich Sie steuern mich hä $\uparrow$ (.) hats einen wichtigen Teil drin äh wo ich mir überlegt habe was ist dann was ist dann eine wichtiger Faktor (.) um Gewalt entgegen (.) zu wirken (.) oder eben die Gewalt fördert und zwar ist das das die erfolgreiche Lebensgestaltung. (.) Ich glaube dass Jugendliche die eine Chance haben eine (.) erfolgreiche Lebensgestaltung (.) zu praktizieren (.) neigen viel weniger zu Gewalt (.) und diejenigen und und und vor allem die die diese Möglichkeit 
nicht haben (.) neigen sehr zu Gewalt (2.5) weil sie wehren sich (.) und sie können es nicht anders." (Zeilen 96-146)

Auf die Frage nach »Gewaltformen« reagiert Herr Tanner, indem er versucht, Gewalt zu definieren. Obwohl er Gewaltdefinitionen zunächst ablehnt, greift er sie dann doch als »Input« auf. Es klingelt an der Tür und Herr Tanner verlässt für einige Minuten den Raum. Nach seiner Rückkehr schließt er an seinen »Versuch«, Gewalt zu definieren, an. Dabei thematisiert er Gewalt auf verschiedenen Ebenen und kommt schließlich auf ein Konzept, das der »erfolgreichen Lebensgestaltung«, zu sprechen, das er als das Mittel schlechthin ansieht, Gewalt zu verhindern. Zunächst grenzt er Gewalt von Macht ab. Gewalt sei etwas anderes als Macht; beides jedoch habe mit unterschiedlichen Mitteln vergleichbare Konsequenzen: »die Realität eines Anderen zu bestimmen«. Im Kontext der offenen Jugendarbeit sei die Unterscheidung zwischen »der tatsächlichen Gewalt zwischen Jugendlichen oder von Jugendlichen gegenüber anderen Altersgruppen« und »Aggression« bzw. »normaler Aggression« grundlegend für sozialpädagogisches Handeln, im Sinne der Entscheidung »wo interveniert man«. Bei »normaler« Aggression sei nicht zu intervenieren, »das händeln die Jugendlichen untereinander«, bei »tatsächlicher Gewalt« hingegen schon. Die Unterscheidung zwischen »tatsächlicher Gewalt« und »normaler Aggression« sei fließend und von verschiedenen Faktoren wie der jeweiligen Situation, den Jugendlichen selbst, den Mitarbeiterinnen bzw. Mitarbeitern sowie dem jeweiligen Ort abhängig. Herr Tanner geht davon aus, dass auch außerhalb der Einrichtung Gewalt eine Rolle spielt, wobei er zwischen Jugendlichen, die seine Einrichtung besuchen, und Jugendlichen, die außerhalb agieren, differenziert. Außerdem geht Herr Tanner davon aus, dass Jugendliche vielfach Opfer häuslicher Gewalt sind. Da es sich bei häuslicher Gewalt jedoch um eine tabuisierte Form von Gewalt handelt, die in der offenen Jugendarbeit nicht thematisiert werde, sei es schwierig, daran anzuschließen. Den Handlungsbedarf (Intervention) begründet Herr Tanner gerade mit der Tabuisierung von häuslicher Gewalt. An dieser Stelle soll festgehalten werden, dass Herr Tanner Intervention als die zentrale und herausfordernde sozialpädagogische Handlungsstrategie in der offenen Jugendarbeit ansieht: Intervention ziele auf »tatsächliche Gewalt « innerhalb der Einrichtung, wobei die Herausforderung darin besteht, in der jeweiligen Situation zu entscheiden, inwiefern ein Eingreifen gerechtfertigt ist. Aggression hingegen sei ein Problem, das Jugendliche selbstständig aushandeln sollen. Die Schwierigkeit, die »Unterscheidung « zu treffen und damit Intervention zu legitimieren, verdeutlicht Herr Tanner in seinen Überlegungen zur Relevanz häuslicher Gewalt: Er sehe diesbezüglich einen Handlungsbedarf, obwohl er keine konkreten Informationen darüber habe. Neben »tatsächlicher Gewalt« und Aggression problematisiert Herr Tanner eine Form struktureller Gewalt, die er anhand der Erzählung über eine Veranstaltung in 
seiner Einrichtung veranschaulicht. Auf dieser Veranstaltung habe die Rede eines SVP-Politikers die Aggression der Jugendlichen geschürt. Herr Tanner übernimmt die Perspektive der Jugendlichen, die immer wieder mit denselben Vorwürfen konfrontiert werden und vor diesem Hintergrund auf die Rede so aggressiv reagiert haben, bis die Veranstaltung eskaliert sei. Diese Eskalation deutet Herr Tanner als Konsequenz einer strukturellen Gewalt, die sich v.a. in Form von Benachteiligung auf dem Bildungs-, Ausbildungs- und Arbeitsmarkt bemerkbar mache. Er nimmt die Perspektive derjenigen Jugendlichen ein, die nicht die Chance hätten, »ein erfolgreiches Leben zu praktizieren«, und daher sehr zu Gewalt neigten. Einer »erfolgreichen Lebensgestaltung« wirke Gewalt also entgegen. Die Interviewerin fragt nach, was unter erfolgreicher Lebensgestaltung zu verstehen sei:

Interviewerin: "Und was was ist eine erfolgreiche Lebensgestaltung was kommt da mit rein was bedeutet des?"

Herr Tanner: "Ähm (.) da gibt es für mich Faktoren wie (.) Selbstbestimmung (.) äh (.) Möglichkeit mitzugestalten mitzuentscheiden mitzuprägen. Das kann in der Familie sein wohin gehen wir in die Ferien äähm wie organisieren wir uns mit den mit den Aufgaben zu Hause (.) aber auch eben die Möglichkeiten (.) dort wo wir ansetzen ansetzen äh Veranstaltungen (.) mitzugestalten und und da geht es nicht um nicht mal nur um die Veranstaltung sondern es geht darum (.) ich (.) meine Veranstaltung ist ausgeschrieben (.) die die prägt das Stadtbild das können die Jugendlichen gar nicht so ausdrücken. (.) Aber ich denke da ist etwas etwas ganz Wichtiges drin das Gefühl zu haben (.) ich mach mit ich beteilige mich (1.5) äh und dann eben auch äh die das Selbstvertrauen das sich daraus entwickelt (.) äh das und und die Achtung (.) vor vor der eigenen Person die Selbstachtung. (2.5) Und und ich glaube auch die Perspektive (.) vor sich vorzustellen ähm ich kann einen Beruf lernen ich habe die Möglichkeit einen Beruf zu bekommen und sich mit dem zu identifizieren (.) etwas (.) etwas ich ich kann etwas tun und sich nicht mit dem identifizieren zu müssen ich kann nichts tun (.) und ich kann Nichts (.) ich bekomme 50 Absagen (.) auch das könnte man als eine Form von Gewalt (.) definieren oder $\uparrow$ (.) wo wo die eigene (.) die die eigene Wahrnehmung und die eigene Selbsteinschätzung dadurch gesteuert wird ich muss mich mit dem konfrontieren und ich muss mich dazu positionieren (.) und das kann in eine depressive (.) ähm a ähm eine depressive Weg nehmen (.) und es kann einen äh einen einen aggressiven (1.5) Weg nehmen (1.0) ich denke so ich hab die die wichtigen Punkte (1.5) mach (.) so (.) aufgezählt." (Zeilen 147-160)

Herr Tanner hat ein konzeptionelles Verständnis von »erfolgreicher Lebensgestaltung «. Sie steht in Bezug zu Autonomie und Partizipation und wird damit auch von der Familie gefördert. Die offene Jugendarbeit fördere »erfolgreiche Lebensgestaltung « in dem Sinne, dass sie den Jugendlichen Möglichkeiten der »Mitbeteiligung « und »Mitgestaltung « zugestehe, woraus Selbstvertrauen und Selbstachtung resultieren würde. Schließlich sei wichtig, dass den Jugendli- 
chen berufliche Perspektiven geboten werden, mit denen sie sich identifizieren können. Gelingt dies nicht, seien Depression oder Aggression die möglichen Folgen. Dieses Konzept enthält vor dem Hintergrund der bisherigen Interpretationen, die das ambivalente Verhältnis von Gesellschaftskritik und Sozialer Arbeit beleuchten, zwei wichtige Aspekte: Erstens reformuliert Herr Tanner seine defizitorientierte Perspektive auf Jugendliche im Kontext offener Jugendarbeit. Zwar übernimmt er die Perspektive Jugendlicher, die gegen gesellschaftliche Benachteiligung rebellieren, dennoch setzt er deren Möglichkeiten der Selbstbestimmung, in einen klar vorgegebenen Rahmen. Die Einrichtung ermögliche es Jugendlichen, Veranstaltungen mitzugestalten. Die Veranstaltungen seien ausgeschrieben und prägten das Stadtbild; »das können die Jugendlichen gar nicht so ausdrücken«. In dieser Gegenüberstellung greift Herr Tanner auf ein Bild zurück, das er bereits in seiner Erzählung über die Entstehung des Bewilligungsverfahren gezeichnet hat: Jugendliche seien aufgrund ihres Mangels an Artikulationsfähigkeit und Erfahrung nicht dazu in der Lage, Veranstaltungen im öffentlichen Raum selbst zu organisieren. Sie müssten legalisierte und institutionelle Angebote der Artikulation und Kommunikation wählen, wie eben das des Bewilligungsverfahrens und der städtisch legitimierten Veranstaltungen. Zweitens dekonstruiert Herr Tanner mit dem Konzept der »erfolgreichen Lebensgestaltung « auch seine gesellschaftskritische Haltung. Das Konzept der »erfolgreichen Lebensgestaltung« ist für ihn gleichbedeutend mit Prävention, im Sinne einer Vorbeugung von Gewalt, wobei er sie nicht aus dem Kontext Sozialer Arbeit heraus entwickelt, sondern als »jugendliche Praktik« beschreibt. In seinem Verständnis von Prävention orientiert er sich also mehr an den Interessen und Vorgaben der Stadt, die eine Anpassung Jugendlicher an die von ihr bereitgestellten Institutionen fordert und fördert, als an den Perspektiven der Jugendlichen. Die Aushandlung der unterschiedlichen Sprecherpositionen, die bereits in den einführenden Interpretationen deutlich wurde, prägt den Interviewverlauf also maßgeblich. Auf die Nachfrage der Interviewerin, woran Jugendarbeiter erkennen könne, ob Jugendliche auf dem Weg »erfolgreicher Lebensgestaltung« seien, führt Herr Tanner ein Beispiel an. Seine Erzählung bezieht sich auf einen Mann, der als Postbote arbeitet. Zufälligerweise handelt es sich um den Postboten, der das Interview kurz vorher durch sein Klingeln unterbrochen hat:

Interviewerin: "Und (.) in Ihrer Erfahrung wie (.) wie sehen Sie (.) des den Jugendliche an sozusagen oder (.) ist des ne Arbeit ähm von von Jugendarbeitern eben des des so zu sehen (.) ob jetzt jemand auf dem Weg is (.) ähm erfolgreich sein Leben zu gestalten oder nicht wie sieht man das?"

Herr Tanner: "Ui (.) das ist ziemlich eine schwierige Frage."

Interviewerin: "Sieht man des? ((lacht))" 
Herr Tanner: "He (3.5) da (.) das ist äh das ist extrem schwierig ähm ich kannte mal einen (1.5) ich hatte mal einen Fußballjunior (.) hehe übrigens der Pöstler (.) der hat mich erkannt (.) ich war sein Trainer (.) als er irgendwie 15 Jahre war (.) oder $\uparrow$ und kommt aus Exjugoslawien (lacht) und-“

Interviewerin: "Echt aha so was ist schön! ((lacht))"

Herr Tanner: "Er ist Pöstler (.) also offensichtlich ich hab inn jetzt sicher äähm was sag ich jetzt mal 1516 vielleicht 20 Jahre nicht mehr gesehen $\uparrow$ (.) ähm (.) und (.) der hat seinen Lebensweg gemacht (.) und (2.0) und (.) er hat mich sogar noch erkannt (.) so (.) oder $\uparrow$. (1.5) Zu der Zeit hatte ich einen Jugendlichen der war top (1.0) der war das Beste was man sich wünschen kann (.) ähm der war erstens gut zweitens sozial drittens eine Führungspersönlichkeit $\uparrow$ (.) äähm (1.5) und ist komplett abgestürzt. (.) Hätten sie mich mit 13 oder 14 gefragt was mit diesem Menschen sein wird hätt ich innen gesagt der macht seinen Weg ich weiß gar nicht ob er lebt also zumindest hat er sicher zwei drei Selbstmord äh Versuche hinter sich (.) und dann gibt es Menschen wie diesen (.) äh wo man sagen kann aus einer Familie aus einer ausländischen Familie schlechter Bildungshintergund und so weiter und die haben ihren Weg gemacht es ist wirklich (.) ganz ganz schwierig das zu sagen (.) auch weil ich denke in diesem Bereich von der Pubertät etwas Entscheidendes passiert da können Kinder die vorher (.) irgendwie still und ruhig und eher so depressiv waren können auf einmal aufblühen und solche die vorher (.) ähm selbstsicher und alles klappt und so (1.5) tauchen einfach ab auf einmal. (.) Meine Tochter im Moment macht eine Krise durch (.) ähm (.) die ist genau dieser Fall (.) perfekt $\uparrow$ (.) die perfekteste Tochter die es gibt (.) und dann kommt sie in die Pubertät wird 16 und dann taucht sie nur noch (.) so (.) oder von dem her find ichs wirklich sehr sehr schwierig (.) was (1.0) was allenfalls (.) daraus sich ableiten lässt ist (.) ähm (.) das lässt sich kaum vom ersten Anblick an (1.5) ähm beurteilen (.) allenfalls Entwicklungen die ein Mensch nimmt (.) und da äh kommt auch etwas wo wo womit die Jugendarbeit (.) vielleicht konfrontiert wird unausgesprochen (.) äh wenn wir sagen (.) wir schaffen präventiv und wir schaffen partizipativ und das gehört zusammen (.) dann hatan das Gefühl ah die beteiligen Jugendliche mit $\uparrow$. (.) Toll die Jugendlichen machen alles (.) oder $\uparrow$ (.) und die Realität ist dann vielleicht dass wir einen (.) einen rein Vornamer haben einen ,ein anderer Vorname، haben der vielleicht (.) nur an einer Sitzung dabei ist (.) keinen Ton sagt (1.0) und an der Veranstaltung dann vielleicht an der Kasse steht (.) oder $\uparrow$ und das ist nichts (.) wenn man irgendwie von Partizipation spricht (.) aber für inn (.) kann es ein unglaublicher wichtiger Schritt sein (.) wie es für inn vielleicht nur ein Schritt war dass er in der Fußballmannschaft ist (.) oder $\uparrow$ ähm (.) und ich denke es es geht wie darum zu sehen was für Schritte machen die Jugendlichen und und und die Entwicklung der Jugendlichen zu beob beobachten und aus dem lässt sich am ehesten sagen (.) äh (.) woo Gewaltpotential ist wie das Entwicklungspotential ist. (.) Ich bin mir jetzt bewusst dass ich Ihnen (2.0) keine Antwort gegeben habe versuchs ab versuchs trotzdem (.) ääh (1.5) ein Merkmal kann sein (.) Jugendliche (.) die (.) aggressiv auftreten (.) in der Sprache $\uparrow$ (.) ähm im in in in der Körperhaltung $\uparrow(1.5)$ kann sein $\uparrow(1.0)$ ist aber genau wicht genauso wichtig zu wissen dass das (.) nicht (.) das dass das nicht unbedingt muss sein (.) es 
können einfach Jugendliche sein die ihre Grenzen austesten und und und die erwarten dass ein Jugendarbeit darauf reagiert. (.) Genauso kann es aber auch sein Jugendliche die sich eher zurückhalten (.) eher ähm (1.0) nicht sprechen (.) sicher eher nicht ausdrücken (.) auch da sollte man genauer hinschauen weil äh (.) auch das muss nicht sein aber auch diese Jugendlichen können allenfalls umgekehrt von Gewalt äh betroffen sein und sich dann des aber auch sehr schnell kann umkehren und sie dann Gewalt ausüben (.) so." (Zeilen 160-196)

An der Schilderung zweier sehr unterschiedlicher Biografien zeigt Herr Tanner, dass »erfolgreiche Lebensgestaltung « letztlich nur retrospektiv zu erfassen ist. Als »Risiken« führt er herkunfts- und bildungsspezifische Merkmale an: Der Postposte komme aus Exjugoslawien, also aus einer »ausländischen Familie schlechter Bildungshintergrund und so weiter«, habe aber trotzdem seinen Weg gemacht. Die Orientierung an diesen Risikomerkmalen sei also nicht verlässlich. Vielmehr sei die Pubertät eine Zäsur in der Entwicklung der Jugendlichen, wie Herr Tanner auch am Beispiel seiner Tochter veranschaulicht. Der Einfluss individueller Merkmale (wie moralische, soziale und »Führungskompetenzen«) sowie der eben genannten herkunftsspezifischen Merkmale könnte in der Pubertät völlig auf den Kopf gestellt werden. Individuelle Lebensverläufe Jugendlicher seien im Kontext offener Jugendarbeit nicht gezielt zu steuern. Über präventive und gleichzeitig partizipative Ansätze könne die offene Jugendarbeit kleine Lern- und Erfolgserlebnisse ermöglichen, die für die Jugendlichen vielleicht einen »unglaublichen wichtigen Schritt« in der individuellen Entwicklung bedeuten. Daher sei es die Aufgabe der Jugendarbeiterinnen und Jugendarbeiter, Jugendliche in ihrer Entwicklung zu beobachten. Sowohl Jugendliche, die aggressiv auftreten, als auch Jugendliche, die sich eher zurückhalten, könnten von Gewalt betroffen sein oder umgekehrt selbst Gewalt ausüben. Aus einer defizitorientierten Perspektive auf Jugendliche, die Herr Tanner in dieser Sequenz weiter bestärkt, differenziert und biologisch begründet, entwickelt er zwei wesentliche Aspekte eines Präventionsverständnisses im Kontext offener Jugendarbeit. Erstens entwickelt er das Konzept der »erfolgreichen Lebensgestaltung « als präventives Konzept im Sinne einer Beobachtungsfolie: Jugendarbeiterinnen und Jugendarbeiter sollten sich nicht auf ihre ersten Eindrücke verlassen, sondern Jugendliche über einen längeren Zeitraum hinweg in ihrer Entwicklung beobachten. Sicherheit könne es nie geben, jedoch können gewisse Einschätzungen hinsichtlich des »Gewaltpotentials« gegeben werden. Letztlich sei »erfolgreiche Lebensgestaltung « und damit der Erfolg der Prävention nur retrospektiv zu beurteilen. Zweitens entwickelt Herr Tanner vor dem Hintergrund einer defizitorientierten Perspektive auf Jugendliche ein Verständnis von Prävention, das darin besteht, Jugendlichen im Rahmen offener Jugendarbeit Partizipation im Sinne von Mitgestaltung zu ermöglichen. Offene Jugendarbeit ist somit als Schutz- und 
Schonraum sowie als Kontrollraum zu verstehen, der es Jugendlichen ermöglicht, ihr Leben »erfolgreich zu gestalten «. Dieses Präventionsverständnis tritt im Kontrast zu Herrn Tanners gesellschaftskritischer Positionierung zutage, nach der auch »die Gesellschaft« in der Verantwortung steht, positiv auf individuelle Lebensverläufe einzuwirken. Demgegenüber steht es in einem kritischen Verhältnis zu einer Risikoorientierung. Herrn Tanners Positionierung zeichnet sich also weiter durch eine Ambivalenz zwischen Gesellschaftskritik und Risikoorientierung aus. Schließlich hält er über eine defizitorientierte Perspektive auf Jugendliche, die er biologisch begründet, eine Normalisierung von Jugendgewalt aufrecht. Wie vereinbart nun Herr Gold seine gesellschaftskritische Perspektive mit einer defizitorientierten Sichtweise auf Jugendliche und welches Verständnis von Prävention entwickelt er vor diesem Hintergrund? Die Interviewerin schließt an seine Ausführungen zur Problematisierung im Sinne einer Kulturalisierung von Jugendgewalt im Kontext seiner schulischen Projektarbeit an:

Interviewerin: "Aha (.) wunderbar des is gut (.) aha (.) dass Sie das sagen ja (.) und (.) wenn Sie ähm jetzt haben Sie auch ein paar Beispiele gebracht $\uparrow$ (.) ähm also welche Formen von Jugendgewalt gibt es eigentlich oder Ihrer Erfahrung nach also was was sind die-«

Herr Gold: "Ähm (.) es sind es sind nach wie vor die klassische Forme von Jugendgwalt $\uparrow$ wo miner Meinig nach aber abgno häd also sprich (.) die physische Forme $\uparrow$ Schlägerei $\uparrow$ ähm. (1.5) Der Punkt is sicher die das es wirklich wos früehner ähm so der der klassische Stand-up Fight ge häd und wen ebbör am Bode gsi isch dann häd ma ufghört. (.) Das isch hüt wirklich nimma so talwies de wird ma einfach aufm Bode entweder aufm Bode witerchämpft (.) oder einer blieb sto und geht ( ) witer oder^ oder mehrere gegen einen und so das isch (.) das hätts früehner in dem Sinn au nüd gea dass sind scho (.) das sind scho neuere Entwicklig wo $\mathrm{n}$ ich denk in de letze zähn füfzähn Johr Fuass äh gfasst händ. (.) Ähm die sind immer no do was ma $r$ au beobachtet isch dass au bei de Maidli oder bei de junge Fraue au mehr zu physischer Gwalt chomt (.) ähm (.) und was natürlich au Klassiker sind (.) sind all die stille (.) äh Gwalttätigkeite wo ma nid so offesichtlich gsit wia Usgrenziga (.) oder alles wos ins Mobbing inneghört Usgrenzuge Beleidigunge was auf auf Facebook passiert und so witer und sehr systemtisch ( ) und äh au schwer halt erfassbar und erchennbar oder $\uparrow$. (.) Ähm also von dem her (.) glaub ich jetzt nöd dass es mega viel neue Entwicklig gäh hätt und ich glaub au nöd dass sich des Feld waahnsinnig groß geändered hätt. (.) Ähm es sind immer no sehr sehr ähnliche Phänomen wo ma eigentlich chönn beobachte (.) und ganz ehlich gsait (.) ich glaube ned dass es in drisg Johr (.) alle Gwalttätigkeite Jugend verschwunde wertdet si sondern (.) es wird immer no (.) es wird immer no da si und man werdet immer no eus mit dem Thema auseinader() (.) nur schon wie s bei de Erwachsene au immer ( ) ganz ehrlich gsait es isch a so." (Zeilen 78-93) 
Herr Gold differenziert zwischen »klassischen« Formen von Jugendgewalt, die er hinsichtlich ihrer problematischen Zuschreibungen entschärft, und »neueren« Formen von Jugendgewalt, die er hingegen problematisiert. Damit hält er die implizite Unterscheidung zwischen »normalen« und »kulturellen« Formen von Jugendgewalt aufrecht. Den »klassischen Stand-up Fight« sowie andere »klassische« Formen von Gewalt wie Mobbing, worunter Herr Gold insbesondere »stille Gewalttätigkeiten« und »Ausgrenzungen« versteht und die für ihn schwer zu erfassen sind, hält er nicht für problematisch. Er problematisiert jedoch die Beobachtung, dass es heute meistens nicht mehr dabei bleibt, sondern »weitergekämpft« wird. Außerdem sei die »physische Gewalt« bei Mädchen angestiegen. Gewisse Beobachtungen problematisiert er also, gibt insgesamt jedoch an, dass sich nichts »wahnsinnig groß geändert« hat und sich auch nichts verändern wird. Jugendgewalt bleibe ein Thema, mit dem sich Erwachsene auch in Zukunft immer auseinanderzusetzen hätten. Diese Einschätzung wirkt vor dem Hintergrund seiner Kritik gesellschaftlicher Dauer- und Überthematisierung von Jugendgewalt resigniert. Herrn Golds Bild von Gesellschaft ist pessimistisch, insofern er die Gesellschaft für eine Veränderung im positiven Sinne nicht als fähig ansieht. Die Interviewerin bedient sich an dieser Stelle einer Leitfrage, die auf die Vermittlung normativer Erwartungen an die Gesellschaft abzielt:

Interviewerin: "Ja (.) eben auch so ne Frage von mir (.) ähm könn also oder stellen Sie sich denn eine Gesellschaft ohne Gewalt vor?"

Herr Gold: „D Frog is ebbe ob ich mir das chann vorstelle und i chan mirs nöd vorstelle nei nei nei nei i ch i cha mas nöd vorstelle. (.) Es es hängt sicher damit zamma ka ok wia def definiert ma Gwalt das is schlussendlich sag $n$ wirs Definitionsproblem oder $\uparrow$. Also (.) ähm aber nei i chan mers nöd vorstelle d es wär es wär utopisch chanz ehrlich gsait es wär utopisch der Mensch hätt (.) hätt ähm (.) hätt die Veralagig für Aggression $\uparrow$. (.) Ähm Aggression kann positiv gnutzt werde (.) Aggression is ned per se eifach (.) schlimm sowie im Sport kann ( ) chanalisiert werde bispielswiese brucht Zeit zum sich chönne (.) teilwis duresetze (.) aber es chann denn halt teilwis ins Gwalttätige kippe und ebbe (.) ( ) gwüsse würdet denn bi ner $n$ Szene sage das isch jetzt no kei Gwalt das isch einfach chli (.) ein heftige Uusanandesezig $n$ Striit ähm und ander sage o das is jetzt scho Gwalt gsi wie mit der Lehrer hätt sie des Machtpotential genutzt und etzt de Schüaler ( ) und ebbe ein Anderer wird sich nei nei also ( ) Tisch klopfe dürfe au mol und so ebbe also es isch wirkli (.) es isch halt sehr ein Definitionssach (.) ähm wenn man jetzt Gwalt sehr (.) eifach allgemein würd definiere nai ich chann mit die Gesellschaft nöd vorstelle ganz ehrlich gsagt chann mir sie nöd vorstelle (.) ich hoffe schon (.) dass es abnimmt aber vorstelle chan ich mir nöd ((lacht))." (Zeilen 94-106)

Herr Gold reformuliert die Frage der Interviewerin, ob er sich eine Gesellschaft ohne Gewalt vorstelle, durch die Frage danach, ob er sich eine Gesell- 
schaft ohne Gewalt vorstellen könne. Herr Gold hält eine Gesellschaft ohne Gewalt für utopisch, was er damit begründet, dass Menschen eine Veranlagung zu Aggression hätten, womit er sein dualistisches Konzept von Gewalt reproduziert. Aggression habe positive Aspekte, könne aber schnell in Gewalt »kippen«. Ähnlich wie Herr Tanner hält er die Unterscheidung für situationsbzw. kontextabhängig, insofern er beobachtet, dass unterschiedliche Gruppen Gewalt auf unterschiedliche Weise definierten:

Interviewerin: "Ja des bringt mich auch gleich zu meiner nächsten Frage (.) ähm (.) also (.) wenn Sie praktisch in inrem Tätigkeitsbereich inwiefern Sie eben äh mit Jugendgewalt zu haben und was Sie tun (.) und tun können ((lacht)) genau."

Herr Gold: »lch schaffe (.) ähm (1.5) viel in der Prävention dass heißt ich mir mir schaffet sehr sehr mit Teams also mit Chlasse hauptsächlich Schualchlasse (.) oder mit irgendwelche Jugendgruppe oder Vereinsgruppe wo denn (.) so Workshop und eventmäßig mit eus schaffet das heißt ich schaffe sehr äh im im im systemisch also mehr mit soziale Syschtem als jetzt mit einzelnen Persone. (.) Ähm ich bin Kerntherapeut ähm ich tun nöd irgendwia äh Schtraftäter oder Gwaltäter denn im den Sinn therapiere sondern probiere eigentlich ähm so klassisch systemtheoretisch halt desch Syschtem z irritiere und luege dass sie sich chönne entwickle und entsprechend Struktrue aufbaue (.) dass es zu weniger Gwalt chumt als dass sie eigentlich cham Syschteme so stabil mache dass sie entweder (.) unterernand ähm i der Chlass füranand eine stönd und einand helfet wenns (.) vo uße her irgendwelche Bedrohig oder Gwaltätigkeite git ähm und au dass sie unterand sich besser chennet lernet und schätze lernet dass es au innerhalb von der Chlass weniger Uusgrenzig $u$ Gwaltätigkeit gibt wil die Erfahrig macht ma oft in der Schul dass halt die meischte Gwaltsache nöd unbedingt die schlimmschte aber immer no die meischte passiert i der Chlass. (.) Uusgrenzige auch chlini Schlägereien uns so witer. (.) Klar auf dem Pausenplatz isch dann oft der Chlassenbegriff aber $d$ Schüler verbringen so viel Zit in ihrer Chlass dass eigentlich die meischte Phänomen Gwaltphänomen in der Chlasse passiertet drum isch mir d Arbeit wirklich mit dem Syschtem Chlass ganz ganz wichtig. (.) Und mit der relevanten Umwelt sprich Lehrer Fachlehrer Schulsoziarbeit wenn möglich au Eltere nur erreicht man die selte (.) ähm also guad (.) dass ähm (.) ja also i schaff wirklich hauptsächlich in dem Bereich (.). Und von dem her isches selten der Fall dass ich konkret (.) ähm Ufträg bekomm wo ma wo ma sait so jetzt so der Gwaltfall passiert mach ebbes (.) sondern ich chomm ( ) und die Themen sind immer latent da das das ma hätt das immer nur (.) es krüzt sich dann au immer sehr mit ähm mit Disziplinarthemen von der Lehrpersone mit Ufmerksamkeitstheme wo d Lehrpersone ribringert ja die Chlass isch gar nöd so schlimm nei sie haben einander eigentlich gern und Gwalt gits eigentlich selte (.) aber sie passen nöd uuf und Disziplin und so und (.) und dös vermischt sich denne so und und denn cha ma eigentlich sehr oft aber ebbe ( ) in der Prävention () sucht ( ) Gwalt ( ) sexuelle Übergriff oder ähm ähm Schulprävention ähm es es sind schlussändlich immer die gliiche ähnliche Schutz- und Risikofaktore wo ma eigentlich möchte a go und behandle ähm wo denn Uswirkige hän uf uf viele von 
dene e Bereiche wo mir halt denn einfach sehr (.) allgemein schaffen also so im Bereich vo $\operatorname{der}($.) äh wie heißt schon wieder tertiäre Prävention auf derer Stufe oder i weiß es nömme ich ich ich woll a lieber lösungsorientiert oder saluto (.) genesisch einen also eifach ((lacht)) lieber lieber a so (.) isch langet das oder äh soll ich no chli mehr verzähln was is was isch frog scho wieder gsi? ((lacht))" (Zeilen 107-131)

Die Interviewerin lenkt die Aufmerksamkeit wieder auf Herrn Golds Tätigkeitsbereich, woraufhin er in seine »professionelle« Perspektive einführt. Als »Kerntherapeut« arbeite er »systemisch«, d.h. weniger mit »einzelnen Personen« als mit Gruppen aus verschiedenen institutionellen und organisatorischen Kontexten. Seine Aufgabe sei es, Strukturen innerhalb dieser Gruppen so zu lenken, dass es dort zu »weniger Gewalt kommt«. Herr Gold begründet seinen Ansatz damit, dass im schulischen Kontext beobachtet wird, dass dort zwar nicht die »schlimmsten«, aber die »meisten« »Gewaltsachen« stattfinden. Die Aufträge, die er erhält, bezögen sich selten auf »Gewaltvorfälle«, sondern meistens auf »latente Themen« bzw. »Disziplinarthemen« oder »Aufmerksamkeitsthemen«. Hier wird deutlich, dass seine Problematisierung von Jugendgewalt an den schulischen Kontext angepasst und von der Nachfrage und Problematisierungsweise seiner Aufraggeber geprägt ist. Daraus resultiert auch sein Präventionsverständnis, das sich »schlussendlich immer« auf »die gleichen Schutz- und Risikofaktoren« bezieht. Dieses Präventionsverständnis, das er als »tertiäre Prävention« bezeichnet, verweist also auf einen Risikodiskurs. Sein »professionelles« Verständnis von Jugendgewalt und Jugendgewaltprävention ist sowohl von seiner gesellschaftskritischen Haltung als auch von seinen »eigenen« Differenzierungen in Bezug auf Jugendgewalt abgekoppelt. Seine Expertise als Kerntherapeut speist sich so gesehen von den Anforderungen von Institutionen der Erziehung und Bildung bzw. den Anforderungen, die diese an Kinder und Jugendliche stellen.

\section{Zum Verhältnis von Gesellschaftskritik und fachbereichsspezifischen Herausforderungen}

Zwischen Herrn Tanner und Herrn Gold lassen sich hinsichtlich der Interviewverläufe wie auch der Argumentationen und Deutungen Parallelen und Unterschiede erkennen. Beide steigen in Abgrenzung zu den Zuschreibungen der Interviewerin mit einer Gesellschaftskritik ein, die v.a. auf die semantische Ebene gesellschaftlicher Thematisierung und Skandalisierung von Jugendgewalt abzielt. Diese klare gesellschaftskritische Positionierung halten sie im Verlauf der Interviews jedoch nicht aufrecht. Je näher sie dem Sprechen-über die für ihre jeweiligen Fachbereiche spezifischen Praktiken kommen, desto weiter entfernen sie sich von einer gesellschaftskritischen Positionierung, woraus sich letztlich eine ambivalente Positionierung zwischen Gesellschaftskritik und Orientierungen im Kontext der jeweiligen fach- und kontextspezifi- 
schen Herausforderungen ergibt. Bemerkenswert ist dabei, dass sie ihre gesellschaftskritische Positionierung aus einer Distanzierung von dem durch die Forscherin zugeschriebenen Expertenstatus entwickeln. Die jeweiligen, aus den fachspezifischen Kontexten heraus entwickelten, Problematisierungsweisen sind von der gesellschaftskritischen Perspektive abgekoppelt. Beide Fachpersonen entwickeln eine defizitorientierte Perspektive auf Jugendliche. Herr Tanner sieht Jugendliche als »Opfer « gesellschaftlicher Diskurse an. Jugendliche hätten nicht die Kompetenzen, sich dagegen zu wehren. Er zweifelt daran, dass Jugendliche die Fähigkeit haben, sich angemessen zu artikulieren, sich selbst zu organisieren und Verantwortung zu übernehmen, was v.a. die Erzählung über das Bewilligungsverfahren zeigt. Erstaunlich ist, dass Herr Tanner das Bewilligungsverfahren nicht deshalb als Erfolg ansieht, weil Jugendliche davon profitieren, sondern Stadt und Polizei. Auch in den Schilderungen aus dem Kontext der offenen Jugendarbeit entwickelt Herr Tanner vor dem Hintergrund seiner defizitorientierten Perspektive ein Präventionsverständnis, das darin besteht, Jugendlichen einen Rahmen für ihre individuelle Entwicklung zu geben. Das Konzept der »erfolgreichen Lebensgestaltung« fungiert somit sowohl als Präventionskonzept als auch als Anspruch an Jugendliche, gesellschaftliche Erwartungen zu erfüllen. Auch Herr Gold hat ein normalisierendes Verständnis von Jugendgewalt, das er nicht nur entwicklungspsychologisch, sondern auch kulturalistisch begründet. Daran entwickelt er jedoch nicht sein Präventionskonzept. Dieses schließt vielmehr an die Erwartungen, Interessen und Problematisierungsweisen seiner Auftraggeber an und begrenzt sich auf schulische Herausforderungen und Kontexte. Zwischen den gesellschaftskritischen Positionierungen und den jeweiligen fachund kontextspezifischen Herausforderungen ergeben sich also Brüche, die auch den Interviewverlauf prägen. Dabei verweisen beide auf Risikodiskurse, wobei sich Herr Tanner davon auch distanziert. Präventionserfolge ließen sich nur retrospektiv ausmachen, wobei er seine Mitarbeiterinnen dazu auffordere, Jugendliche über einen längeren Zeitraum zu beobachten. Diese Unterschiede lassen sich hinsichtlich der unterschiedlichen Kontexte - offene Jugendarbeit und Schule - plausibilisieren, wie der Vergleich mit anderen befragten Fachpersonen zeigt. Herr Auto, der auch mit Schulklassen arbeitet, bindet seine Expertise an die gewachsene Nachfrage zurück. Dabei übernimmt er die Problematisierungsweisen seiner Auftraggeber und entwickelt daran sein Präventionsverständnis. Frau Schock, die u.a. von Schulen damit beauftragt wird, in Familien zu gehen, muss an Problematisierungsweisen, die sich bereits etabliert haben, anschließen. Dies reflektiert und hinterfragt sie, wobei sie dies ohne Probleme in ihre Expertise integrieren kann. Frau Blaum stellt, wie auch Herr Tanner, die Bedeutung der Beziehungsarbeit heraus, worauf nachfolgend zurückgekommen wird. Ein Präventionskonzept wie das der »erfolgreichen Lebensgestaltung« benötigt einen regelmäßigen und mehr oder weniger in- 
tensiven Kontakt mit Jugendlichen. Die Gemeinsamkeiten lassen sich anhand des Bezugs zu gesellschaftlichen Diskursen sowie Problem- und Risikodiskursen plausibilisieren. Beide Präventionskonzepte zielen letztlich auf eine Anpassung der Jugendlichen an gesellschaftliche Erwartungen und Normen ab. Die Anforderung einer »erfolgreichen Lebensgestaltung « scheint nur dann realisierbar, wenn Jugendliche auf gesellschaftlich etablierte Strukturen wie institutionalisierte Angebote und Kommunikations- bzw. Artikulationsformen zugreifen. Auch in schulischen Kontexten geht es um die Vermittlung des Anspruchs, lern- und leistungsförderliche Strukturen zu schaffen. Herr Tanner und Herr Gold können Gesellschaftskritik im Kontext ihrer Arbeit nicht »zu Ende denken«. Zwar hinterfragen und kritisieren sie Gesellschaft, vertreten diese jedoch auch als erwachsene Schweizer und setzen in ihren Lösungen nicht an Gesellschaft an, sondern an den Jugendlichen, die sich anzupassen haben. Eine defizitorientierte Perspektive auf Jugendliche stellt gewissermaßen das Bindeglied zwischen Kritik und fachspezifischen Herausforderungen dar. Die bisher vorliegenden Interpretationen eröffnen die Frage, inwieweit sich Gesellschaftskritik aus Kontexten Sozialer Arbeit heraus denken und realisieren lässt, was in der theoretischen Diskussion aufgegriffen wird. Eine gesellschaftskritische Positionierung erfolgt in der Distanzierung von den Zuschreibungen hinsichtlich der Expertise der befragten Fachpersonen durch die Interviewerin und kann als implizite Abgrenzung zu kriminalpolitischer Programmatik gedeutet werden, insofern sich die Interviewerin eines Problemdiskurses bedient. Auch unabhängig von den konkreten Leitfragen vermittelt das Forschungsvorhaben gegenüber den Befragten eine bestimmte Erwartungshaltung, die auf Problemdiskurse verweist. Anhand der Erzählungen und Schilderungen ihrer Arbeitsfelder entwickeln Herr Tanner und Herr Gold Problematisierungsweisen und Präventionsverständnisse, die mit ihrer gesellschaftskritischen Perspektive brechen und sich an kriminalpolitischer Programmatik orientieren. Das Bindeglied zwischen den unterschiedlichen Positionierungen, davon geht die hier vorliegende Arbeit aus, stellt eine defizitorientierte Perspektive auf Jugendliche dar. Wie ist der Zusammenhang zwischen Gesellschaftskritik und Defizitperspektive im Kontext offener Jugendarbeit zu denken? Die gesellschaftskritische Haltung Herrn Tanners bricht nur scheinbar mit dessen defizitorientierter Perspektive auf Jugendliche; vielmehr entwickelt er sie vor diesem Hintergrund und leitet seine Expertise davon ab. Jugendliche könnten sich gegenüber gesellschaftlicher Skandalisierung, Diskriminierung und Benachteiligung nicht wehren, da sie nicht über die angemessenen Kompetenzen und Mittel der Artikulation und Kommunikation verfügten. Aus diesen Gründen würden sie weder über die Fähigkeit verfügen noch die Verantwortung dafür übernehmen, sich selbst zu organisieren. Aus dieser Perspektive auf Jugendliche entwickelt Herr Tanner nun seine eigene Expertise als leitender Jugendarbeiter im Kontext offener Jugend- 
arbeit. Diese fördere Jugendliche in ihrer individuellen Entwicklung bis hin zur »erfolgreichen Lebensgestaltung«. Seine Einrichtung biete einen Schutzund Schonraum für Jugendliche, in dem sie Erfahrungen machen können, die ihnen bei der Realisierung eines »erfolgreichen Lebens« helfen könnten. Die Einrichtung kompensiert somit fehlende familiäre Aufgaben. Seine defizitorientierte Perspektive auf Jugendliche, die er über den Rekurs auf eine biologistische Deutung von Jugendgewalt normalisiert, kann somit als konstitutiv für sein Verständnis von offener Jugendarbeit angesehen werden. In dieser Legitimationsweise verliert Herr Tanner jedoch den Anschluss an eine gesellschaftskritische Haltung, da sein Präventionskonzept auf die »erfolgreiche« Anpassung Jugendlicher an gesellschaftliche Anforderungen abzielt. Seine Gesellschaftskritik löst sich in der Forderung an die Jugendlichen, sich gesellschaftlichen Erwartungen anzupassen, sich zu normieren, auf. So wird aus einem fürsorgerischen Ansatz eine Forderung, die von Jugendlichen eingelöst werden muss. Weiter gedacht liegt die Verantwortung für eine »erfolgreiche Lebensgestaltung« bei den Jugendlichen selbst. Herrn Tanners Gesellschaftskritik sind also Grenzen gesetzt, die von den Rahmenbedingungen und Zugzwängen der offenen Jugendarbeit vorgegeben sind, und löst sich in ihnen auf. Im schulischen Kontext scheint die Möglichkeit, an gesellschaftskritische Positionierungen anzuschließen, noch schwieriger zu sein, was an der Abhängigkeit von konkreten Aufträgen sowie der Geschlossenheit von Schule und Schulklasse, deren Besuch (im Gegensatz zur offenen Jugendarbeit) nicht freiwillig ist, liegen mag. Herr Gold normalisiert Gewalt vor dem Hintergrund einer Kritik an gesellschaftlichen Skandalisierungs- und Pathologisierungsprozessen. Dabei rekurriert er nicht nur auf biologistische und entwicklungspsychologische, sondern auch auf kulturalistische Argumentationen. Diese Kritik und die damit verbundenen Deutungen von Jugendgewalt werden in schulischen Kontexten jedoch nicht relevant. In seiner Arbeit mit Schulklassen hat Herr Gold die Aufgabe, schulischen Interessen gerecht zu werden, also Strukturen zu schaffen, die Disziplin und Aufmerksamkeit und damit Lernen und Leistung fördern. Implizit legitimiert er seine Expertise über Nachfrage.

\section{Bezüge zur kriminalpolitischen Programmatik}

Herr Tanner und Herr Gold entwickeln aus ihrer Gesellschaftskritik heraus eine defizitorientierte Perspektive auf Jugendliche, die sie entwicklungspsychologisch, biologistisch, historistisch und kulturalistisch begründen, womit sie Jugendgewalt letztlich normalisieren. Dabei (re-)produzieren sie einen Dualismus zwischen der Gesellschaft und den Jugendlichen - »Kultur« und »Natur«. Die Jugendlichen seien der Gesellschaft mehr oder weniger schutzlos ausgeliefert. Die Präventionskonzepte beider Befragten zielen nun jedoch nicht auf die Veränderung gesellschaftlicher Strukturen ab, sondern auf die Anpassung der Jugendlichen an die gesellschaftlichen Strukturen - nicht ohne 
der Gesellschaft gegenüber eine gewisse Resignation auszudrücken. Die Präventionsansätze unterscheiden sich in ihrem Bezug auf die kriminalpolitische Programmatik. Herr Tanner steht dieser kritischer gegenüber, wobei daran erinnert werden soll, dass Prävention sich in beiden Ansätzen nicht auf »pathologische« Formen von Jugendgewalt bezieht. Die Unterscheidung zwischen »normalen« und »pathologischen« Formen von Jugendgewalt scheint für die konkreten Praktiken nicht relevant zu sein. Dabei distanziert sich Herr Tanner ganz konkret von der Zielsetzung des »Nationalen Präventionsprogramms Jugend und Gewalt«.

Herr Tanner: "(...) ähm aus auslösen äh es geht eigentlich darum dass dass wenn interveniert wird (2.0) ja de de wenn die Polizei interveniert geht's geht's geht's um Recht und Ordnung und Strafe. (.) Wenn wir intervenieren (.) dann (.) ist es mir ein Anliegen dass immer ein Lernansatz dahinter ist (.) unsere Intervention also außer es geht darum um um um Leben zu schützen oder $\uparrow$ (.) aber sonst geht ein geht eine eine Intervention muss immer einhergehen mit einem Lernschritt (.) mit einem Lernprozess und deshalb müssen Interventionen (.) aus meiner Sicht (.) individualisiert werden es gibt es gobt bei uns keine stä Standard (.) wenn das dann das (.) sondern wenn das $\uparrow$ (.) und die Situation des Jugenlichen (.) wird eine Intervention gemacht als Situation der Jugendlichen (.) wird eine Intervention gemacht also wir wir wirklich intervenieren im Sinn $\uparrow$ (.) dieser Schritt ist Intervention und Prävention zugleich und es geht darum dass die Jugendlichen sich auseinandersetzen mit dem was sie tun $\uparrow$ (.) mit dem was sie bei anderen auslösen (.) ähm und mit dem wie sies wieder gut tun können (.) für sich und für andere so das denk ich sind so zentrale Elemente. (Zeilen 324-332)

Herr Tanner erkennt eine klare Differenz zwischen Jugendarbeit und Polizeiarbeit, insbesondere hinsichtlich ihrer Ziele und Adressierungsweisen. Dabei spezifiziert er auch sein Verständnis von Intervention, das sich, wie sich auch im folgenden Kapitel zeigen wird, nicht klar von Prävention unterscheidet. Intervention und Prävention im Kontext von Jugendarbeit zielten darauf ab, Jugendlichen »Lernschritte« zu ermöglichen und diese zu unterstützen. Daher müsse Intervention »individualisiert werden«. Diese Aussage kann in Abgrenzung zu dem »Standard, den es bei uns nicht gibt«, und vor dem Hintergrund der aktuellen Präventionspolitik als implizite Kritik an diesbezüglichen Standardisierungsbestrebungen gelesen werden und damit als Bedrohung eines Präventionsverständnisses, das sich an individuellen Bedürfnissen der Entwicklung, des Lernens und der Bildung orientiert. Der Aspekt der Kontrolle tritt hier zugunsten des Aspektes der Hilfe zurück. Jener ist aus der Perspektive Herrn Tanners eine polizeiliche Angelegenheit. Die Ambivalenz zwischen Hilfe und Kontrolle und den damit verbundenen Konflikten zwischen Jugendarbeit und Polizei wird im folgenden Kapitel tiefergehender thematisiert. Eine ähnliche Beobachtung trifft auf die Schilderungen Frau Blaums zu. Hier wird 
im Folgenden aufgezeigt, dass Prävention im Kontext von Jugendarbeit durch die Orientierung an von außen herangetragenen Erwartungen irritiert werden kann, was auch eine Bedeutung aus der Perspektive Jugendlicher haben kann. Frau Blaum arbeitet, wie bereits erwähnt, in derselben Einrichtung, in der Herr Tanner leitend tätig ist, als Jugendarbeiterin in einem Quartier. In der bereits einleitend interpretierten Einstiegspassage wird deutlich, dass Frau Blaum versucht, einen Präventionsauftrag durch die Stadt zu erfüllen. Vor diesem Hintergrund entwickelt Frau Blaum ein Präventionsverständnis, das als Gewalt verhindernde und »nachhaltige« Maßnahme zu verstehen ist. Dies sieht sie im Kontext der offenen Jugendarbeit als nicht realisierbar an, insbesondere da aufgrund der Rahmenbedingung der »Freiwilligkeit« die Reichweite des Einflusses auf die Jugendlichen und deren Lebenswelten durch die Jugendarbeiterinnen und Jugendarbeiter begrenzt ist. Sie werde erst »aktiv, wenn Gewalt da ist«. Diese Aussage greift die Interviewerin auf:

Interviewerin: "Und also wann ist Gewalt da? (.) Was (1.0) woran sieht man des?" Frau Blaum : „Ich kann innen ein Beispiel geben ((lacht)). Vor einem Monat hatte ich mit einem Jugendlichen (.) eine Diskussion zum Thema Religion. (.) Äh ich habe inm gesagt ich sei Atheistin und und blaba was auch immer oder und dann hab ich es argumentiert und er natürlich mit seinen Argumenten er ist äh (.) gläubig und nach einem Zeitpunkt hab ich bemerkt er kann nicht weitergehen mit seinen Argumenten er ist immer dann mit Koran gekommen wo ich dann schlussendlich als (.) Buch in Frage gestellt habe. (.) Hab ich inm alles weggenommen oder $\uparrow$. (.) Und dann hab ich bemerkt er wird (.) wütend er wird lauter ich habs einfach beobachtet oder $\uparrow$. (.) Und dann bin ich weiter und er hat mir dann (.) nicht mehr zugehört (3.0) und (.) plötzlich hat er einfach den äh Stuhl genommen Gegenstände genommen und einfach rumgeworfen und dann den Sofa getreten was auch immer oder $\uparrow$. (.) Das war dann so seine Reaktion (.) da hab ich mich gefragt (.) was war das jetzt? (2.0) ( ) er kann nicht zuhören (.) er kann nicht diskutieren (.) er kann nicht argumentieren (.) ähm (2.0) und wenn er dann zu einem Punkt kommt wo er nicht weiter (.) geht oder gehen kann (.) kommts einfach des einzige was er hat äh (.) und das sind halt Sozialkompetenzen die fehlen oder äh (.) das war klar oder $\uparrow$ und (.) und ja." Interviewerin: "Hmhm. (1.0) Und was haben Sie dann gemacht?"

Frau Blaum: „Ich hab weiter mit inm diskutiert oder gesprochen natürlich nicht über des Thema sondern über sein Verhalten da hab ich inm gesagt (.) weißt Du (.) es ist auch nicht so schlimm wenn Du sagen kannst da hast Du nicht genug Kenntnisse du möchtes da Dich weiter (2.0) bilden lesen und und dass wir dann in einem Monat nochmal darüber diskutieren also Wissen ist auch keine (.) Schande Du kannst auch sagen du äh ich bin stecken geblieben ich komme nicht weiter äh und da hätt ich auch aufgehört oder so ich habe dann nachher versucht inm (.) zu zeigen dass er sein Verhalten selber reflektieren kann äh. (.) Ob ich da Erfolg habe weiß ich nicht ob er dann nachher sein Verhalten ändern wird aber es war sehr interessant für mich ja."

Interviewerin: "Ok." 
Frau Blaum: "Hmhm"

Interviewerin: "Und haben Sie danach nochmal darüber gesprochen oder ist des nochmal-"

Frau Blaum: "Nein. (2.5) Jetzt ist er nicht mehr bei uns aber ich hab Informationen von inm von den anderen Kollegen die bei uns verkehren äh. (1.5) Er geht jetzt (.) in ein Fitnesszentrum. (2.0) Ähm (.) er ist jeden Tag dorten seine Freizeit und er versucht sich so (.) Muskeln zu bauen und es geht in die Richtung eben dass er dann äh (.) in jeder Situation in der er ein Problem hat (.) seinen Körper einsetzen kann es geht wirklich in die Richtung äh (.) äh es ist schade aber es ist so ((lacht))" (Zeilen 40-65)

Frau Blaum entwickelt eine Erzählung: Sie habe mit einem Jugendlichen, der gläubig sei, eine Diskussion zum Thema Religion gehabt, wobei sie in ihren Argumentationen die Oberhand gewonnen habe. Dem Argument des Jugendlichen, das sich auf den Koran bezogen habe, habe sie jegliche Gültigkeit abgesprochen, indem sie den Koran »als Buch in Frage gestellt« habe. Anschließend habe der Jugendliche seine Stimme erhoben, worauf hin Frau Blaum »weiter sei«. Der Jugendliche habe ihr dann nicht mehr zugehört, mit Gegenständen geworfen und das Sofa getreten. Dies interpretiert Frau Blaum dahingehend, dass er nicht in der Lage gewesen sei, mit ihren Argumenten umzugehen: Er könne nicht zuhören, er könne nicht diskutieren, er könne nicht argumentieren; ihm fehlten die »Sozialkompetenzen«. An der Darstellungsebene fällt auf, dass Frau Blaum ihre eigene Beobachtung sukzessive bewertet und somit ihrer Perspektive als Beobachterin derjenigen der Handelnden deutlichen Vorrang gibt. Die Betonung ihrer beobachtenden, bewertenden und vorhersehenden Haltung, die sie mit den Aussagen »das war ja klar« und »es war sehr interessant für mich « in besonderer Weise festigt, suggeriert, dass während des Geschehens etwas Relevantes zu beobachten und vorherzusehen gewesen sei: das Gewaltverhalten des Jugendlichen aufgrund seiner fehlenden »Sozialkompetenzen«. Damit deutet sie Jugendgewalt als »Verhalten«, das als Reaktion auf die Spiegelung einer Niederlage resultiert, die sie wiederum aus einem individuellen Defizit heraus erklärt. Diese Deutung läuft während ihrer Erzählung im Hintergrund mit, womit sie ihr Wissen über Jugendgewalt an der erzählten Situation abarbeitet und reproduziert. Dies ermöglicht ihr wiederum, das »Verhalten« des Jugendlichen letztlich auch tatsächlich als Gewalt zu deuten. Daraufhin fragt die Interviewerin, was Frau Blaum dann gemacht habe. Frau Blaum gibt an, weiter mit dem Jugendlichen gesprochen zu haben - »natürlich nicht über des Thema, sondern über sein Verhalten«. Sie habe ihm dabei aufgezeigt, dass es in Ordnung sei, nicht genug zu wissen, um mit ihr zu diskutieren zu können, er aber die Möglichkeit habe, sich »weiter zu bilden« und schließlich noch einmal mit ihr zu diskutieren. Außerdem könne er sein eigenes Verhalten reflektieren. An diesen Schilderungen ist bemerkenswert, dass Frau Blaum weniger das »Verhalten« des Jugendlichen selbst problematisiert, 
sondern seine fehlenden »Sozialkompetenzen«. Damit problematisiert sie das, was das »Verhalten« von Jugendlichen ihrer Beobachtung nach auslöst. Ihre »Intervention« bestehe dann darin, dem Jugendlichen seine Defizite transparent zu machen. Sie gibt an, letztlich nicht zu wissen, ob sie damit »Erfolg hatte «, was sie damit plausibilisiert, dass der Jugendliche die Einrichtung seitdem nicht mehr besucht habe. Relevant an dieser Darstellung ist, dass Frau Blaum ihr Deutungswissen über das »Gewalthandeln « von Jugendlichen und dessen Entstehung nicht nur reproduziert, sondern gleichzeitig individualisiert und zwar in einem doppelten Sinne: Sie projiziert ihr Wissen in die erzählte Situation, indem sie es dieser als Deutungsfolie zugrunde legt. Damit verkörpern der Jugendliche und sein Handeln dieses Wissen. Zweitens »entlässt« sie den Jugendlichen mit dem Auftrag, sich nach ihren normativen Kriterien weiterzubilden. Diese Anforderung ist Teil der Expertise Frau Blaums in Bezug auf Intervention und Prävention in der offenen Jugendarbeit. Ihre Intervention plausibilisiert sie an der Beobachtung, dass Gewalt einfach da sei. Die Notwendigkeit ihrer Intervention verknüpft sie dann mit einem Präventionsbestreben, das in der Forderung an den Jugendlichen besteht, sich weiterzubilden. Die Interviewerin fragt, ob Frau Blaum noch einmal mit dem Jugendlichen darüber gesprochen habe, was sie mit der Begründung verneint, dass der Jugendliche nicht mehr bei ihnen sei. Nach Informationen »anderer Kollegen« verbringe er jeden Tag im Fitnesszentrum. Vor dem Hintergrund ihrer Beobachtung, dass er keine Sozialkompetenzen habe (nicht zuhören, nicht diskutieren und nicht argumentieren könne), deutet sie seinen »Versuch, sich Muskeln zu bauen «, als Problemlösungsstrategie, die sie abwertet. Damit impliziert sie eine Differenz zwischen mit Gewalt assoziierter Körperarbeit und Einsichtigkeit, Reflexion und Wissenserwerb. Letztere seien »richtige « Strategien, um Probleme zu lösen und Gewalthandeln vorzubeugen. Ihr eigenes Handeln hinterfragt sie hingegen nicht, problematisiert auch das Wegbleiben des Jugendlichen nicht und vertraut auf die Informationen der übrigen Jugendlichen. Frau Blaums Perspektive ist in ähnlicher Weise wie Herrn Tanners und Herrn Golds durch eine defizitorientierte Perspektive geprägt, was sie hier zunächst an einem Beispiel darstellt und schließlich verallgemeinert. Sie erklärt das »Gewalthandeln« des Jugendlichen anhand seiner defizitären »Sozialkompetenzen «, fehlenden Einsichtigkeit und Bildungsbestrebungen. Sie sieht es als ihre Aufgabe an, dem Jugendlichen ihre Sichtweisen zu erläutern, damit er sein Verhalten reflektieren und sich weiterbilden kann und in dem Sinne ihre Sichtweisen annimmt. Damit vermittelt sie ihm einen Bildungsauftrag. Prävention versteht sie damit als eine Aufgabe, die der Jugendliche selbst zu erfüllen hat und zu der sie ihn nur auffordern kann. Dieses Präventionsverständnis ähnelt dem von Herr Tanner, der das Konzept der »erfolgreichen Lebensgestaltung « als Anleitung in die Hände der Jugendlichen legt und angibt, diese bei der Realisierung nur unterstützen zu können. Frau Blaum orientiert sich 
in ihrer Erzählung und deren Deutung an dem Wissen, das sie über jugendliches »Gewaltverhalten « hat und reproduziert und individualisiert dieses dabei. Damit orientiert sie sich implizit an Risikofaktoren, die sie auf das konkrete Beispiel projiziert. Herr Tanner plädiert hingegen dafür, am individuellen Fall anzusetzen. Diese Unterschiede können auf unterschiedliche Darstellungsweisen bezogen werden, die zum Teil den Interaktionsverläufen der Interviews unterliegen. Das Interview mit Frau Blaum beginnt im Unterschied zu den Interviews mit Herrn Tanner und Herrn Gold nicht mit der ursprünglich gewählten Einstiegsfrage, sondern mit einer offenen Erzählaufforderung, die Narrationen begünstigt. Die »Einstiegsfrage« wird nachgelagert:

Interviewerin: "Wenn Sie des so auf den Punkt bringen müssten wie sehen Sie denn generell also des Problem (1.5) ähm von Jugendgewalt?"

Frau Blaum: "Also ich hab jetzt aktuelle Zahlen nicht aber wie wir so lesen heißt es irgendwelche Jugendgewalt nimmt zu^ (.) ähm wo ich es bei meiner Arbeit nicht bestätigen kann äh (.) wo ich mich frage wenn es wirklich die Realität wäre dann sollten wir auch bei uns oder in unserer Umgebung etwas spüren (.) aber es kann ich nicht bestätigen. (2.0) Äh was ich aber sagen kann (.) wenn ich höre also ich bin seit zwei Jahren da (.) in dem Treff aber seit zehn Jahren in dem Beruf ich habe vorher in Name einer Stadt Jugendarbeit gemacht. (1.5) Ich kann nur zwei Beispiele geben wo ich Gewalt bei uns im Treff wirklich erlebt habe das waren zweimal Schlägerein äh unter zwei Gruppen. (.) Das sind die die zwei einzigen Erfahrungen wo ich wirklich von Gewalt sprechen kann oder $\uparrow$. (2.0) Und und da im Treff ist es eigentlich selten der Fall (.) dass Jugendliche aufeinander losgehen (1.5) oder irgendwie Gewalt spürbar ist. (.) Was ich aber höre oder mitbekomme das findet draußen statt das erzählen dann die Jugendlichen oder $\uparrow$. Die sind dann als Gruppe irgendwie in 'Name eines Stadtteilsı gegangen und dann haben sie sich dort mit einer Gruppe getroffen und dann aufeinander losgegangen. (.) Das sind so Erzählungen ich weiß nicht ob es stimmt (.) oder in dem Maß stimmt wie sie es erzählen aber hier im Raum haben wir das nicht. (1.5) Äh (2.5) aber anscheinend dass kann ich dann auch (.) eben durch diesen Hören sagen äh bestätigen dass es dann heftiger wird also dass sie wenn sie schlagen dann werden sie irgendwie (.) äh brutaler also äh (.) da kommen noch Gegenstände wie Messer und Zeug äh ins Spiel ähm (.) äh sie sind dann vorbereitet sie gehen vorbereitet dorthin mit Gegenständen es ist nicht so dass es dann irgendwie von einer Situation (.) ähm (.) so zum Punkt kommen dass sie dann aktiv werden sondern sie sind vorbereitet. (.) Äh und dass ist für mich ein Punkt äh was mir bedenken macht oder also äh." (Zeilen 66-81)

Frau Blaum bearbeitet die »Einstiegsfrage« auf andere Weise als Herr Tanner und Herr Gold. Die offene Erzählaufforderung ermöglicht ihr zunächst eine Kontextualisierung relevanter Aspekte ihres Fachbereichs - der offenen Jugendarbeit. Sie verknüpft die Einstiegsfrage weniger mit der Adressierung als Expertin als im Sinne einer Zuständigkeit dafür, »gute Präventionsarbeit« zu 
leisten. Die offene Erzählaufforderung ermöglicht ihr mehr Spielraum für die Entwicklung eigener Verständnisse von Jugendgewalt und Jugendgewaltprävention. Frau Blaum beschreibt hier drei verschiedene, miteinander konkurrierende Perspektiven in Bezug auf die Problematisierung von Jugendgewalt: statistisch generiertes Wissen, die eigene berufsbiografische Erfahrung und die Erzählungen der Jugendlichen über das, was außerhalb der Einrichtung geschieht. Den »aktuellen Zahlen« und den Erzählungen der Jugendlichen gesteht sie insofern einen Vorrang vor ihren eigenen berufsbiografischen Erfahrungen zu, als sie diese an jenen validiert. Die Information, Gewalt nehme zu, kann sie weder »bestätigen« noch »spüren«. Gegenüber den Erzählungen der Jugendlichen, dass es außerhalb der Einrichtung »heftiger « und »brutaler« werde, hegt sie zunächst Zweifel, könne es schließlich aber doch bestätigen. Die Unsicherheit in Bezug auf ihre eigene Einschätzung weist einerseits auf eine hohe Außenorientierung, andererseits auf eine hohe Innenorientierung hin, die sie miteinander verbindet. Sie selbst erfährt nur das, was innerhalb der Einrichtung passiert, und dort sei Gewalt selten. Sie verlässt sich auf die Informationen der Jugendlichen, die den Treff aufsuchen. Dies spricht dafür, dass die Vertrauensbeziehung zu den Jugendlichen für sie basal ist, was sie im Folgenden spezifiziert.

Interviewerin: "Und wie reden Sie dann mit den Jugendlichen darüber (3.0) wenn sie des erzählen?"

Frau Blaum: (4.0) Einerseits (.) ist das ja so wir sind für sie (.) Vertrauenspersonen Beziehungspersonen unsere Arbeit basiert auf Beziehungen (.) und (.) wenn sie mir (.) solche Dinge erzählen ähm (.) dann find ich es gut das wir informiert sind dass sie es nicht verstecken weil das dürfen sie ja den Eltern nicht erzählen das dürfen sie ja den Lehrern nicht erzählen. (.) Ähm es sind auch andere Dinge oder wenn sie etwas klauen einfach was sie sonst in der Schule und Familie nicht machen dürfen (.) und trotzdem es machen und und das uns erzählen das find ich sehr positiv äh da ist ein Vertrauen da (.) äh und dadurch suchen sie eigentlich auch die Diskussion oder sie möchten (.) von uns wissen was wir darüber denken (.) äh dann sprech ich mit denen und versuche denen andere Lösungsweisen zu zeigen wie man auch ein eine Problem auch sonst lösen kann. (.) Ähm (2.5) aber meine Haltung ist nicht in der ersten Linie ja dann wird ich jetzt aktiv dann wird ich jetzt Polizei informieren oder Jugenddienst informieren (.) irgendwelche Maßnahmen äh (.) m mit dem komm ich dann (.) eher als als letztes ähä ich versuche mit denen im Dialog zu bleiben (.) ich frage dann nachher eine Woche später ist da jetzt was passiert und und und hat die andere Gruppe jetzt auch Kontakt aufgenommen wie geht es. (.) Ich bin einfach am Laufenden. (.) Äh natürlich je nach Grade äh überleg ich mir auch ob ich etwas äh unternehmen soll eben (.) Fachstellen informieren soll aber äh solange es in diesem Rahmen bleibt mache ich das nicht (.) hmhm hmhm weil ich ich denke sobald ich das einmal mache (.) dann werden sie mir nachher mir davon nichts 
erzählen (.) und auch äh Vertrauensbasis wird auch gebrochen und und und dann kann ich mit denen nicht arbeiten." (Zeilen 82-104)

Auf die Frage der Interviewerin, wie Frau Blaum mit den Jugendlichen redet, wenn sie ihr von Gewalt erzählen, begründet sie ihre »Expertise «über die Vertrauensbeziehung zu den Jugendlichen. Diese stelle die Basis ihrer Arbeit dar: Würde die »Vertrauensbasis« gebrochen werden, könne sie nicht mehr mit den Jugendlichen arbeiten. Diese Vertrauensbeziehung erweckt den Eindruck einer Art Bündnisses, das Frau Blaum und die Jugendlichen gegen Schule und Familie eingehen. Die Jugendlichen könnten ihr Dinge erzählen, die sie anderen nicht erzählen könnten. Frau Blaum deutet dies dahingehend, dass die Jugendlichen die Diskussion mit ihr suchten und wissen wollten, was sie darüber denkt. Frau Blaum versucht dann, die Jugendlichen bei der Problemlösung zu unterstützen. Die Alternative wäre, andere Institutionen wie die Polizei oder den Jugenddienst einzubeziehen, was Frau Blaum zu vermeiden versucht, weil dies den Dialog abbrechen würde und damit die Grundlage ihrer Expertise - die Vertrauensbeziehung - zerstören würde. Damit problematisiert Frau Blaum das Konfliktfeld der offenen Jugendarbeit und der Polizeiarbeit und verweist damit auf einen grundlegenden Konflikt, den auch Herr Tanner schildert: Frau Blaums Präventionsverständnis steht im Widerspruch zu dem von der Stadt formulierten Auftrag, worauf sie bereits in der Einstiegssequenz hinweist. Prävention als nachhaltiges und messbares Konzept, das den Einbezug von Eltern und Schule benötige, sei unter den Bedingungen des gegenseitigen Vertrauens und der Freiwilligkeit nicht realisierbar. Ihr eigenes Präventionskonzept bestehe v.a. im Dialog mit den Jugendlichen, der nicht abbrechen dürfe und in dessen Rahmen Frau Blaum den Jugendlichen Hilfestellungen und Bildungsaufträge geben könne. Hinsichtlich ihres Präventionsverständnisses deutet Frau Blaum jedoch auf Unsicherheiten hin. Beispielsweise äußert sie Zweifel an ihrem eigenen Gespür und den Informationen der Jugendlichen vor dem Hintergrund statistisch generierten Wissens. Vor dem Hintergrund ihres Präventionsverständnisses lässt sich ihre Erzählung sowohl als misslungene Intervention als auch als misslungene Prävention deuten, insofern als der Dialog abgebrochen ist. Dies sieht Frau Blaum jedoch nicht als Misslingen, sondern als Bestätigung ihrer eigenen Annahmen an: Der Jugendliche bleibe uneinsichtig und kompensiere seine Defizite hinsichtlich seiner »Sozialkompetenzen« im Fitnesszentrum, wo er sich »Muskeln baut«. Auch fürchtet sie den Vertrauensverlust der Jugendlichen, wenn sie mit anderen Institutionen kooperiert. Die Interviewerin fragt daraufhin nach den Grenzen der Intervention:

Interviewerin: "Hmhm und wie würden Sie diese Grenze beschreiben dass Sie dann (.) doch mal des Jugend äh des Jugendamt informieren oder den Jugendanwalt?" 
Frau Blaum: "Es ist so es sind halt die Jugendlichen oder und sie versuchen auch über ihre Grenzen zu gehen es sind Banalitäten oder $\uparrow$. (.) Irgendein Jugendlicher hatte (.) von einem die Freundin irgendwie schräg angeschaut äh ähm (.) und und dann dann (.) das ist das Problem oder $\uparrow$ äh (.) und und ich denke sie sollen auch diese Erfahrungen machen bis zu einem Grad oder äh klar wäre es besser dass es ohne Gewalt mit einer Erklärung mit einer Diskussion mit einem Gespräch (.) was auch immer äh (.) lösen könnten äh (.) aber das ander ist auch ok äh (.) ähä." (Zeilen 105-112)

An die Frage der Interviewerin, die auf die Grenzen abzielt, die dazu führen, dass Frau Blaum andere Maßnahmen ergreift, schließt Frau Blaum in nicht erwarteter Weise an. Sie verbindet mit dem Begriff der Grenze ein Grenzen überschreitendes Handeln Jugendlicher, das sie normalisiert: »So sind halt die Jugendlichen«. Sie erläutert, dass diese Grenzen schon sehr tief ansetzten, beispielsweise könne es für einen Jugendlichen ein Problem sein, dass die Freundin »schräg angeschaut« werde. Gewalt im Sinne von Gewalthandeln könne zwar eine erfahrungsreiche Strategie der Problemlösung sein, sei aber die schlechtere Alternative. Damit stellt Frau Blaum wiederum das Bild von Gewalt und Dialog gegenüber, womit sie ihre defizitorientierte Perspektive reproduziert. Im folgenden Interviewverlauf nimmt Frau Blaum, ähnlich wie Herr Tanner und Herr Gold, eine gesellschaftskritische Positionierung ein:

Interviewerin: "Und ähm (.) woran glauben Sie liegt es dass die Gewalt so härter geworden ist oder so brutaler wie Sie gesagt haben?"

Frau Blaum: "Ich glaube es spielen sicher Medien eine eine Rolle (1.5) auch so einfacher Zugang zu den Mitteln äh sei es Spiele (.) äh in dem Bereich bin ich nicht so gut weil ich weiß nicht was es alles gibt äh (1.0) ähm (1.0) das ist sicher ein Faktor. (7.0) Auch wie Medien mit dem umgeht wie wie (.) wie Medien darüber berichtet glaub ich ist auch ein Faktor. (3.0) Und des andere ist natürlich es hat immer mit dem zu tun (.) dass die Jugendliche mit der Perspektivlosigkeit von diesem Jugendlichen also die (.) die (2.0) Chancengleichheit wir immer weniger. (9.0) Ja ähä." (113-119)

Die Interviewerin kommt auf Frau Blaums Beobachtung zurück, nach der Gewalt vehementer geworden sei. Dies könne sie hinsichtlich ihrer Erfahrungen innerhalb der Einrichtung nicht bestätigen, glaube aber den Erzählungen der Jugendlichen über das, was »draußen« stattfindet. Frau Blaum verknüpft diese Beobachtung mit der Rolle der Medien, sowohl was die ihre Nutzung betrifft als auch ihre Berichterstattung, sowie damit, dass Perspektivlosigkeit und Chancenungleichheit zugenommen hätten. Hier positioniert sich Frau Blaum also kritisch gegenüber der medialen Berichterstattung sowie dem Medienkonsum und nimmt die Jugendlichen, in ähnlicher Weise wie Herr Tanner, in Schutz. Im Folgenden führt sie ihre Kritik weiter aus: 
Interviewerin: "Und ähm wie berichten die Medien darüber wie hat sich entwickelt?" Frau Blaum: "Also ich hab das Gefühl es wird (.) es gibt so eine Tendenz (.) erstens (.) in dem Bereich jetzt kommt es zum Punkt eben Jugendgewalt äh (.) is eigentlich Ausländerproblem oder Problematik was auch immer. (2.0) Ähm. (3.0) Also die Jugendliche mit Migrationshintergrund darüber wird (.) erst berichtet wenn sie dann eben äh (1.0) Gewalt ausüben also im negativen Sinne alles was im negativen Sinne passiert wird berichtet aber (.) äh (.) das sind auch die gleiche Jugendliche die auch äh äh (.) Stärken haben äh Qualitäten haben andere Dinge machen zwar sehr gut machen aber darüber wird nicht berichtet oder sehr selten (.) das ist mal das eine und das andere ist (.) es wird immer so detailliert (.) berichtet (.) es geht sehr wahrscheinlich darum dass dass (1.0) Medien berichte so rüberbringen muss dass sie dann interessant sind und gelesen werden aber es wird immer so im Detail äh berichtet wie ein Vorfall Zwischenfall im Bereich Gewalt einfach passiert ist das ist mir aufgefallen äh." (Zeilen 120-129).

Frau Blaum führt hier ihre Kritik der medialen Berichterstattung weiter aus, wobei deutlich wird, dass sie über einen ganz ähnlichen Erfahrungshintergrund verfügt wie Herr Tanner und Herr Gold. Sie kritisiert die Medien dahingehend, dass diese die Themen Jugendgewalt und Migration miteinander verknüpfen und dadurch ein negatives Bild von Jugendlichen, die über einen »Migrationshintergrund « verfügen, transportieren. Dies sei einseitig, da diese Jugendlichen auch »Qualitäten« hätten, worüber jedoch nicht berichtet werden würde. Sie kritisiert, dass Vorfälle in den Medien detailreich geschildert werden. Nun kommt die Interviewerin auf die Gewaltvorfälle zurück, die in der Einrichtung geschehen.

Interviewerin: "Welche Formen von Gewalt (.) mit welchen Formen von Gewalt haben Sie hier in der Einrichtung zu tun?"

Frau Blaum: "Äh Mobbing ist ein Thema natürlich oder $\uparrow$. (2.0) Ähm. (5.5) Der Arbeitsbereich mit den Schulen zusammen äh wenn in den Schulklassen Jugendliche gemobbt werden (.) tun wir mit der Schulsozialarbeiterin zusammen anschauen was wir machen können. Wir haben zum Beispiel einen Monat mit einer Klasse so einen Workshop gemacht zum Thema Mobbing (.) wo die Jugendlichen da waren und und ja. (2.5) Und (.) das ist sicher mal eine Form und das andere verbal oder $\uparrow$ äh das haben wir auch aber das hat eben mit dem zu tun was ich vorher gesagt habe (.) sie haben diese Kultur nicht (.) äh (.) Sprech und Diskussionskultur nicht äh (.) ähm sobald irgendetwas gesagt wird was denen nicht passt werden sie laut äh (2.0) es wird geschimpft und und (.) ja. (1.0) Aber ich komm immer zu diesem Punkt oder $\uparrow$ also sie (.) ich frage mich auch (.) das sind zum Teil Jugendliche die hier geboren sind und hier aufgewachsen sind. (1.0) Was macht dann (.) und hier in die Schule gegangen sind natürlich oder $\uparrow$. (1.5) Was macht dann die Schule frag ich mich die ganze Zeit wenn die Jugendliche mit 17 (.) 18 nicht mal die Kompetenzen haben (.) äh ein Gespräch zu führen oder zu diskutieren äh." (Zeilen 130-140) 
Mobbing sei »natürlich« eine häufige Form von Gewalt. Diesbezüglich arbeite die Einrichtung mit der Schulsozialarbeit zusammen. Davon grenzt Frau Blaum »verbale Gewalt« ab. Diese führt sie darauf zurück, dass die Jugendlichen »diese Kultur« bzw. »diese Sprech- und Diskussionskultur« nicht hätten. Dies betreffe zum Teil auch Jugendliche, die in der Schweiz geboren und aufgewachsen sind. Damit verknüpft Frau Blaum ihre defizitorientierte Perspektive auf Jugendliche mit einer kulturalistischen Deutung, indem sie an die in der Schweiz geborenen und aufgewachsenen Jugendlichen andere Erwartungen formuliert als an Jugendliche, die nicht in der Schweiz geboren und aufgewachsen sind. Gleichzeitig nimmt sie diese Jugendlichen in Schutz, da sie die Schule für deren Mangel an »Kompetenzen« verantwortlich macht. Auffällig ist, dass Frau Blaum die Frage nach Formen von Gewalt hier primär problem- und lösungsorientiert bearbeitet. Sie beschreibt nicht die Formen von Gewalt, sondern die problematischen Formen von Gewalt, deren Ursachen sie zu ergründen sucht und für die es Lösungen braucht. Diese erwartet sie von der Schule. Ihre Problem- und Lösungsorientierung kann auch auf die Interaktionssituation und die Fragen der Interviewerin zurückgeführt werden. Die Forscherin bringt einen Problemdiskurs ein, den Frau Blaum aufgreift. Der Interaktionsverlauf und die damit verbundenen Positionierungen verändern sich mit den unterschiedlichen Strategien der Interviewerin, Themen einzubringen. Auf die offene Erzählaufforderung hin schildert Frau Blaum die Angebote und Abläufe im Kontext der offenen Jugendarbeit in »neutraler« Weise. Hier zeigt sich, dass sie eine kritische Positionierung gegenüber der Schule und den Medien entwickelt, aber im Sprechen über problematische Formen von Gewalt nicht aufrechterhält und nicht weiter ausführt. In Verbindung mit ihren kritischen Argumenten stellt sie Jugendliche als schutzbedürftig dar. In Bezug zu »Gewaltvorfällen« hingegen hebt sie deren Defizite hervor. Auf diese Weise nimmt sie Jugendliche zwar grundsätzlich in Schutz, problematisiert letztlich jedoch deren Handeln bzw. die Defizite, die ihrer Beobachtung nach dieses problematische Handeln begünstigen. Frau Blaum entwickelt in Bezug auf Jugendgewaltprävention ihre Expertise, Jugendlichen auf Basis einer Beziehungs- und Vertrauensebene Möglichkeiten der Bildung, des Kompetenzerwerbs und Strategien der Problemlösung zu vermitteln. Dies basiert auf der Konstruktion von Jugendgewalt als ein Handeln, das aus einem biologisch und kulturell begründeten Defizit heraus resultiert. In ihren Erzählungen und Beschreibungen tritt Gewalt v.a. als physische Gewalt in Form reaktiver Handlungsweisen und Körperarbeit auf, die als Kompensation sozialer, sprachlicher und Bildungsdefizite und nicht als ernstzunehmende Problemlösungsstrategie anzusehen ist. Ihre Beziehungs- und Vertrauensarbeit sowie ihr Bestreben, den Jugendlichen soziale und sprachliche Kompetenzen sowie Bildungsaufträge zu vermitteln, grenzt sie ab von Prävention als Gewalt verhinderndes und nachhaltiges Konzept, das messbar und ausweisbar ist, jedoch 
als von der Stadt gegebener Auftrag erfüllt und nachgewiesen werden muss. Diesen Präventionsauftrag kann sie aufgrund von Restriktionen der offenen Jugendarbeit, wie v.a. der Vertrauensbeziehung zu den Jugendlichen, der Freiwilligkeit und des fehlenden Wissens über die familiären Hintergründe der Jugendlichen, nicht erfüllen. Somit stehen sich aus Frau Blaums Perspektive zwei Präventionsverständnisse gegenüber, die sie nicht integrieren kann. Frau Blaum verweist auf eine Bedrohung ihres eigenen Präventionsverständnisses durch einen Präventionsauftrag, der auf Wirksamkeit und Nachhaltigkeit abzielt und mess- und darstellbar sein soll. Jedoch stößt Frau Blaum hinsichtlich ihres eigenen Präventionsverständnisses an Grenzen: Der Dialog zu dem Jugendlichen ist abgebrochen; sie versucht, ihre eigenen Erfahrungen an den Informationen der Jugendlichen und statistisch generiertem Wissen zu validieren; ihre defizitorientierte Perspektive geht mit einem Misstrauen gegenüber den Jugendlichen einher; eigene, von den Jugendlichen praktizierte Lösungen wie z.B. religiöse Praktiken oder Bodybuilding erkennt sie nicht an.

In den Interpretationen dieses Kapitels deuten sich bereits zentrale Orientierungsmuster der befragten Fachpersonen an. Das Sprechen über Jugendgewaltprävention weist deutliche Ambivalenzen auf, was sich insbesondere am Bruch zweier unterschiedlicher Positionierungen zeigt: Eine gesellschaftskritische Positionierung steht einer defizitorientierten Perspektive gegenüber, aus der an Jugendliche gerichtete Anpassungserwartungen abgeleitet werden. Die Integration dieser beiden Perspektiven erfolgt über einen Legitimationsmechanismus: Jugendgewaltprävention erfährt über eine defizitorientierte Perspektive eine Rechtfertigung. Herr Tanner und Herr Gold sind bestrebt sowohl den Interessen einer Mehrheitsgesellschaft, der sie sich auch selbst zurechnen, als auch den Interessen der Jugendlichen, die sie wiederum in Schutz nehmen, gerecht zu werden. Frau Blaum hingegen situiert sich v.a. zwischen den Interessen ihrer Auftraggeberin und den Interessen der Jugendlichen und den im Quartier lebenden Familien. Aber auch sie entwickelt im Interviewverlauf eine gesellschaftskritische Perspektive, die ihre Defizitperspektive auf Jugendliche insofern unterstützt, als diese sie dazu berechtigt, Jugendliche in Schutz zu nehmen. Letztlich lösen sich die gesellschaftskritischen Positionierungen der Befragten zugunsten einer Legitimation ihrer Präventionsarbeit auf. Devianzkonstruktionen betreffen nicht die Handlungsebene, sondern die Defizite, die als Erklärung für problematisch wahrgenommenes Handeln herangezogen werden. Gewalthandeln wird dabei gleichzeitig normalisiert, damit Präventionsarbeit, die sich auf das Schutzbedürfnis Jugendlicher bezieht, dauerhaft durchgesetzt werden kann. Die Gesellschaftskritik fungiert hier als Bedrohungsszenario der Jugendlichen. Insofern begründen die befragten Fachpersonen ihr Präventionsverständnis über die Konstruktion eines Schutzbedürfnisses Jugendlicher, das in ihrer Perspektive sowohl aus biologischen 
und kulturellen Gegebenheiten resultiert als auch aus einer gesellschaftlichen Bedrohung. Diese Form der Legitimierung betrifft Soziale Arbeit im Kontext eines Zuständigkeitskonflikts mit

"anderen Instanzen der Devianzregulierung [...]. Die Soziale Arbeit ist vor diesem Hintergrund herausgefordert, ihren Zugriff auf Devianz zu begründen. In Bezug auf die jeweils erfassten Problembereiche muss sie einen Interventionsbedarf herausstellen und deutlich machen, dass dieser sich nur adäquat im Rahmen dieses Handlungsfeldes bearbeiten lässt." (Schmidt 2015: 106f.)

Die Normalisierung jugendlichen Gewalthandelns ist dabei eine grundlegende Strategie, die auf die Möglichkeit abzielt, Devianz dauerhaft zu bearbeiten, wie im Folgenden weiter ausdifferenziert wird.

\subsubsection{Zur Normalisierung von Jugendgewalt}

Der Begriff der »Normalisierung« von Jugendgewalt verweist auf zwei Beobachtungen: Zum einen verweist er darauf, dass die befragten Fachpersonen Jugendgewalt im Sinne eines Gewalthandelns als ein für die Jugendphase nicht veränderbares Handlungsmuster deuten und als solches verallgemeinern. Dies begründen sie biologisch, historisch, psychologisch, kulturell und biografisch. Zum anderen verweist der Begriff auf die Beobachtung, dass jugendliches Gewalthandeln normalisiert werden soll, in dem Sinne, dass Jugendliche, wenn sie erwachsen sind, diese Handlungsmuster nicht mehr zeigen. Diese Logik mutet paradox an, weist aber auf die Bedeutung der Legitimation der Professionen Sozialer Arbeit hin, die sich an einer Orientierung an Präventionsdiskursen nährt. »Der Normalisierungsauftrag setzt Normalitätsvorstellungen voraus, die sich auf Erwartungen von Normallebensläufen und auf (historisch-) spezifische Lebensmodelle beziehen [...].« (Dollinger/Oelkers 2015: 10) Die Interviews weisen darauf hin, dass die Normen bzw. Normalitätserwartungen der Fachpersonen vorstrukturiert sind: durch Beteiligte wie der Stadt, der Polizei oder der Öffentlichkeit, durch eigene biografische Erfahrungen, durch Annahmen darüber, was eine »gute« bzw. »erfolgreiche« Lebensweise auszeichnet. Die Fachpersonen rekurrieren auf die Notwendigkeit »abweichenden Verhaltens « in der Jugendphase. »Jugendliche gelten als Individuen, für die die herrschenden Normen und Werte einer Gesellschaft nicht selbstverständlich sind oder keine Gültigkeit haben und an denen soziale Kontrolle demonstriert werden muss.« (Althoff 2002b: 76) Aus einer defizitorientierten Perspektive heraus werden Jugendlichen also nicht nur Fähigkeiten und Kompetenzen abgesprochen, sondern sie werden von bestimmten Normalitätserwartungen in der Gegenwart befreit. Somit zielen die hier rekonstruierten Präventionsverständnisse zwar auf die »Verhinderung von Normabweichung« und sind als 
»korrigierender Zugriff auf die Person bzw. die Überwachung und Kontrolle der Entwicklung junger Menschen auf der Grundlage gesellschaftlicher Normalitätsanforderungen und Normvorgaben « (Stehr 2009: 117) zu verstehen, jedoch in dem Sinne, dass sich die Korrekturen auf ein »normales« jugendliches Gewalthandeln beziehen. Die Möglichkeit, »normale« Jugendgewalt zu verhindern, würde die Professionen Sozialer Arbeit in ihrer Existenzberechtigung bedrohen. In diesem Sinne kann die Normalisierung von Jugendgewalt als Präventionsstrategie angesehen werden, die auf die Sicherung der Professionen (und damit auch der generationalen Ordnung) abzielt. Nicht die Annahme, Jugendgewaltprävention würde Bestehendes verändern, trägt den Diskurs, sondern die Annahme, Jugendgewaltprävention würde Bestehendes nicht verändern. Das Risiko, auf das sich Jugendgewaltprävention, hier verstanden als »vorbeugende Kontingenzbewältigung « (Bröckling 2002: 42), bezieht, liegt also darin, jugendliches Gewalthandeln könnte sich in Zukunft verändern - nicht jedoch nur im Sinne einer Steigerung oder eines Anstiegs, sondern ebenso im Sinne einer Abschwächung oder eines Rückgangs. Im Folgenden wird der Zusammenhang zwischen den Problematisierungsweisen von Jugendgewalt und daran anschließenden Präventionsverständnissen und damit das Verständnis bezüglich der Orientierungsweisen der Fachpersonen im Kontext von Jugendgewaltprävention an kriminalpolitischer Programmatik vertieft. Die Interpretationen erfolgen nun primär themenbezogen, wobei an die vorangegangenen Interpretationen angeschlossen wird und bestimmte Fälle herausgestellt werden. Sowohl die Interpretationen der Einstiegspassagen als auch die weiterführenden Interpretationen deuten darauf hin, dass sich die befragten Fachpersonen vor dem Hintergrund des an sie herangetragenen Anspruchs, präventiv im Sinne einer Wirksamkeitsorientierung und Nachhaltigkeit zu arbeiten, tendenziell distanzieren - eine Beobachtung, welche auf diejenigen Fachbereiche, die den Charakter von Dienstleistungsorganisationen aufweisen, weniger zutrifft als auf Kontexte »klassischer« Sozialer Arbeit. Es ist festzustellen, dass einige der Fachpersonen verschiedene Präventionsverständnisse als nicht miteinander kompatibel ansehen, wie v.a. an den Schilderungen Frau Blaums und Herrn Tanners ersichtlich wird. Diese Beobachtungen werden im Folgenden vertieft wie auch die damit in Zusammenhang stehende Beobachtung, dass die Normalisierung von Jugendgewalt mit einer defizitorientierten Perspektive auf Jugendliche einhergeht, die damit als schutzbedürftige soziale Gruppe adressiert wird. Die »Normalitätsarbeit« ist damit nicht »im Sinne einer helfenden oder auch kontrollierenden Anpassung devianter Individuen an die Normen der Gesellschaft« (Dollinger/Oelkers 2015: 11) zu verstehen, sondern bezieht sich auch auf die soziale Gruppe der Jugendlichen. Zunächst wird an die Interpretationen des Interviews mit Frau Blaum angeschlossen: Frau Blaum entwickelt ein eigenes Präventionsverständnis, das darauf abzielt, Jugendlichen im Rahmen der offenen Jugendarbeit, Möglich- 
keiten des Kompetenz- und Bildungserwerbs zuzugestehen, was sie durchaus auch einfordert, indem sie dies als Auftrag an die Jugendlichen (re-)adressiert. Ihr Präventionsverständnis grenzt sie vom Präventionsauftrag der Stadt ab, der auf Nachhaltigkeit und Evidenz abzielt. Dieser torpediert das ihr eigene Präventionsverständnis insofern, als er die Substanz ihrer Arbeit angreifen würde: die Vertrauensbeziehung, die v.a. vom Vertrauen der Jugendlichen ihr selbst gegenüber geprägt ist.

Interviewerin: "Ähm (3.0) können Sie vielleicht nochmal etwas dazu sagen (.) Jugend und Gewalt (.) wie hängt das zusammen?»

Frau Blaum: (13.0) "Ääh (4.0) also (2.5) wie ich vorher gesagt habe Jugendlich in dem Alter (.) dass sie probieren ihre Grenzen (2.5) zu sehen oder zu überschreiten (.) also Gewalt kommt ja auch unter Erwachsenen aber auch unter den Kindern also Kindern oder es ist nicht nur ein ein Thema was nur unter Jugendlichen vorkommt. (1.5) Der Punkt ist da (.) es gibt gewisse Jugendliche die in dem Alter gewalttätig werden aber dann nachher mit dem aufhören im Erwachsenenalter $\uparrow$. (.) Es gibt aber Jugendliche (.) die (.) mit dem nachher auch weiter machen (.) im Erwachsenenalter. (4.0) Und (.) da ist die Frage oder was passiert da bei einigen Jugendlichen bei denen es nur ein ein äh (2.0) $\mathrm{s}$ Jugend (.) wie sagt man dem (.) äh (.) Thema bleibt und bei dem anderen nicht. (3.0) Äh da da hab ich keine Antwort da kommt es sehr wahrscheinlich auch so äh so persönliche Entwicklungspunkte rein äh. (6.0) Jugend und Gewalt also es ist für mich nicht ein Bereich automatisch was zusammengehört äh ja." (Zeilen 200-109)

Auf die Frage nach dem Zusammenhang von Jugend und Gewalt rekurriert Frau Blaum zunächst auf eine »entwicklungspsychologische« Perspektive auf Jugendgewalt, indem sie jugendliche Handlungsmuster auf deren altersbedingtes Entwicklungsstadium zurückführt. Davon grenzt sie ihre Beobachtung, dass Gewalt auch »unter Erwachsenen aber auch unter den Kindern vorkommt«, ab. Anschließend problematisiert sie die Beobachtung, dass es Jugendliche gibt, die im Erwachsenenalter »mit dem weiter machen «, und Jugendliche, die dies nicht täten. Erklären könne sie das nicht, sie verweist aber auf Unterschiede in der »Persönlichkeit«. Schließlich nimmt sie direkt zu der Frage der Interviewerin Stellung, dass Jugend und Gewalt Bereiche seien, die für sie nicht automatisch zusammengehören. Damit differenziert Frau Blaum zwischen Jugendgewalt, die sie normalisiert und damit legitimiert, und Gewalt, die sie problematisiert und damit in Abgrenzung zu Jugendgewalt pathologisiert: Bestimmte Handlungsmuster seien für die Jugendphase normal. Bestimmte Jugendliche würden diese Muster jedoch auch im Erwachsenenalter zeigen. Hierin zeigt sich eine Parallele zu Herrn Tanner, der es nicht für möglich hält, die Entwicklung Jugendlicher in Bezug auf eine »erfolgreiche« Lebensgestaltung einzuschätzen. Dies könne erst retrospektiv beurteilt werden. Auch zeigt sich eine Parallele zu Herrn Unterwasser, der sich in Rahmen 
seiner Tätigkeit sehr intensiv mit der Frage auseinandersetzt, was »er einem männlichen Menschen antun müsste [...] damit er dann mit zwanzig ein ausgewachsener Gewalttäter ist«, und damit die Irreversibilität einer »erwachsenen Täterkarriere « betont. Problematisiert wird hier Gewalthandeln, das Jugendliche ausüben, wenn sie schon erwachsen sind. Dies erscheint paradox, erlangt aber an Plausibilität, wenn die Restriktionen der jeweiligen Rahmenbedingungen bedacht werden, denen die Fachpersonen verhaftet sind. Innerhalb dieser Rahmen reagieren sie vielmehr auf Jugendgewalt im Sinne einer Intervention und setzten sich weniger mit den Bedingungen, Möglichkeiten und möglichen Wirkungen von Prävention auseinander. Oft wird Intervention erst im Nachhinein als Prävention umgedeutet. Dieses Spannungsverhältnis von Intervention und Prävention wird im Folgenden von Herrn Tanner und Herrn Unterwasser beschrieben:

Interviewerin: "Hmhm (.) hmhm (.) gut (.) was mich auch interessiert ist einfach Ihrer Erfahrung und Ihre Sicht (.) also (.) genau wollt ich nur nochmal sagen. (1.5) Ähm Sie haben schon zwei Begriffe genannt die mich weiter interessieren das ist Intervention und Prävention (.) ist des Beides Bestandteil (.) Ihrer Arbeit oder der Arbeit der Name der Einrichtungr?"

Herr Tanner: " Ja (.) Beides. (.) Beides ist Bestandteil und es geht in (.) es geht es es es ist es ist äh fließend (.) äh eigentlich sehen wir uns (2.0) sehen wir uns als präventiv (.) also unser Ziel ist es präventiv zu arbeiten (.) ähm. (1.5) Erstens geht das nicht immer (.) also (1.0) es kann Vorfälle kommen wo wo des einfach wo wo es eine Interven wo es eine Intevention braucht $\uparrow$ (.) und zweitens äh haben wir ges sa sag ich ja die Jugendlichen müssen lernen $\uparrow$ also können sie Fehler (.) machen (.) also müssen wir auch präventiv arbeiten können (.) also a müssen wir auch präventiv inter inter äähm inter äh intervenieren so. (1.5) Äh es kommt auch drauf an (.) wo man das sieht (.) ähm bei wenn ich jetzt zum Beispiel jetzt um Drogen das ist denk ich das beste (.) Beispiel wie arbeiten präventiv im Sinn dass die Jugendlichen (.) mit dem nicht so gefährlichen kann man ja auch mal testen aber (.) aber aber aber nicht so viel oder ein ein guten Umgang damit zu

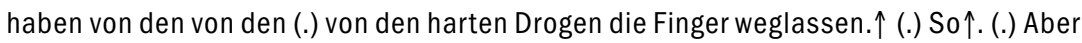
man trifft vielleicht auf Jugendliche die zu uns kommen oder die halt nach zwei Jahren (.) Jahren wirklich zu viel Drogen konsumiert oder Alkohol konsumieren und auch dort kann man präventiv arbeiten als wir sehen das wirklich als als fließenden (.) fließende Geschichte (.) äh äh wir haben zwar eine Nullgewalttoleranz in unseren Regeln $\uparrow$ (.) aber wir gehen davon aus dass es das Jugendliche halt kommen und dann ist Gewalt da $\uparrow$ und dann muss man intervenieren und präventiv arbeiten so." (Zeilen 232-246)

Sowohl Prävention als auch Intervention seien Bestandteile seiner Arbeit, wobei die Grenzen fließend seien. Eigentlich sei es ihr Ziel, präventiv zu arbeiten, jedoch sei es manchmal eher angebracht zu intervenieren. Damit schildert Herr Tanner in ähnlicher Weise wie Frau Blaum eine Kluft zwischen dem An- 
spruch und Selbstverständnis, präventiv zu arbeiten, und der Notwendigkeit, zu intervenieren, wodurch die Realisierung von Prävention als primäres Ziel in den Hintergrund rückt. An dieser Stelle führt er eine in der Theorie klare Unterscheidung von Intervention und Prävention ein: Intervention sei eine notwenige Reaktion auf bestimmte »Vorfälle« und Prävention ein Lernprozess, in dem Jugendliche etwas ausprobieren und auch Fehler machen dürften. Intervention und Prävention stehen so gesehen in einem Spannungsverhältnis zueinander. Grundsätzlich versteht Herr Tanner die offene Jugendarbeit als Lernfeld, das Jugendlichen den Raum lässt, etwas auszuprobieren, etwa »die nicht so gefährlichen Drogen«. Seiner Erfahrung nach sei jedoch davon auszugehen, dass auch Jugendliche kommen, die viel konsumieren oder Gewalt provozieren. Dann müsse man interventiv und präventiv arbeiten. Diesen Interventionsdruck schildert auch Frau Blaum, die ebenfalls davon spricht, dass Gewalt da sei. Dieses Verständnis offener Jugendarbeit, das sowohl Herr Tanner als auch Frau Blaum vertreten, unterstützt den Moratoriumsgedanken bezüglich der Jugendphase, dem jedoch auch Grenzen zu setzen sind. Ein bestimmtes Ausmaß an Drogenkonsum oder Gewalt sei nicht zu tolerieren und erfordere Intervention. Das Spannungsverhältnis von Intervention und Prävention entwickelt Herr Tanner also aus dem Handlungskontext seines Tätigkeitsfeldes - der offenen Jugendarbeit - und nicht aus einer Definition der Begriffe oder einer damit verbundenen Ziel- oder Wirkungsorientierung. Prävention und Intervention sind grundlegende Orientierungspunkte der offenen Jugendarbeit, deren (Be-)Deutungen Herr Tanner im Kontext bzw. am jeweiligen Fall entwickelt. Frau Blaum setzt das Spannungsverhältnis von Intervention und Prävention hingegen sehr viel stärker in den Kontext eines an Nachhaltigkeit und Evidenz orientierten »Präventionsauftrags«, was es noch zusätzlich verkompliziert. »Ergebnisse« erschließen sich laut Herr Tanner und Frau Blaum nur retrospektiv und nur am individuellen Fall, sofern der Kontakt zu den Jugendlichen bzw. den inzwischen Erwachsenen besteht. Beispielsweise habe Herr Tanner nicht damit gerechnet, dass ein Jugendlicher, den er früher begleitet habe, Postbote geworden sei und damit sein »Leben erfolgreich gestaltet habe«. Frau Blaum hat keinen Kontakt mehr zu dem Jugendlichen, mit dem sie einen Konflikt hatte, erkennt ihre »Intervention« aber nicht als Scheitern an, sondern zeigt sich in ihrer kulturalistischen und biologistischen Deutungsweise bezüglich der Entstehung jugendlichen Gewalthandelns vielmehr bestätigt und reproduziert diese damit. Prävention bemisst sich aus der Perspektive der beiden Fachpersonen also weniger anhand der Beobachtung, dass (jugendliches) Gewalthandeln entsteht oder nicht entsteht, sondern vielmehr der Beobachtung, dass sich ihre normativen Erwartungen hinsichtlich der jeweiligen Lebensverläufe oder Einstellungen erfüllt haben oder nicht. Das Wirken von Prävention erschließt sich Herr Tanner über seine Annahme, dass eine Person, die als Postbote arbeitet, ein erfolgreiches Leben habe. Diese An- 
nahme geht möglicherweise mit der Annahme einher, ein festes Einkommen zu haben und eine vertrauensvolle Arbeit mit der Post anderer auszuführen, seien Zeichen eines erfolgreichen Lebens. Frau Blaum deutet ihre »Intervention « nicht als Scheitern, sondern als Bestätigung ihrer Annahme, dass dem Jugendlichen schlicht die Sozialkompetenzen und die Bildung fehlen, um zu einer angemessenen Einstellung gegenüber der Religion bzw. dem Koran zu gelangen. In dieser Lesart beurteilen Frau Blaum und Herr Tanner ihre Erfolge also hinsichtlich ihrer eigenen normativen Erwartung daran, was ein »gutes« Leben ist oder was eine »gesunde« Einstellung zur Religion ausmacht. Auch Herr Unterwasser schlingert bei der Abgrenzung von Intervention und Prävention, was er als Dilemma bezeichnet, hat aber für sich eine Lösung gefunden.

Interviewerin: "Und jetzt haben Sie grad ein Beispiel genannt das war ja eigentlich schon eher so Intervention ähm wo sind bei Innen die Schwerpunkte bei Intervention oder Prävention oder kann man des überhaupt so trennen?"

Herr Unterwasser: "Ja cha man nöd ähm ((lacht)). Es isch ja so dass mini Fachstell (.) a Trägerschaft hät wo mir ein klaren Uftrag erteilt hät nämlich (.) präventiv wirksam z si (1.5) und hat sich dann aber ganz schnell zeigt die wo zu mir chömmet also Schuele Gmeinde (.) Verwaltige Behörde (1.5) äh Polizeie wenn die zu mir chömmet dann händs es Problem und das sollt bis Morn glöst si. (2.5) Und da gahts nöd um Prävention oder $\uparrow$ da da ghats allefalls um Prävention fürs nächscht mal also ebbe da isch wirklich da da isch schon schwäre Körperverletzig passiert da is schon s Berhördemitglied bedroht worde mit Mord zum Teil mehrmals scho bis dann d Lüt wirklich reagieret. (.) Und (.) am Afang bin ich dann wirklich in nem Dilemma gschtanda mittlerwile han ich nehm ich das locker $\uparrow$. (.) Ich sag dem eifach d frühe Intervention oder (lacht) oder deklariers als Prävention und und find für mich so dasch is Prävention für Zukunft mal egal was scho passiert isch es is immer no Prävention (.) ich ich ich versuch das nimmer z trenne aber aber faktisch (.) das Thema brucht $\mathrm{n}$ Liedensdruck damit s Lüt apacket das das isch eifach so und und is mis Dilemma au (.) jetzt grad bi Chind und Jugendliche die chömmet zu mir wenns schon z spat isch oder $\uparrow$ also wenn wenn scho wenn scho ein ein erheblicher Schade in der Biographie angrichtet isch das isch eifach a stück wit so. (1.0) Es isch halt tru trurig bei de Jugendliche (.) bei de meischte Jugendliche wo zur Jugendawaltschaft chömmet (.) die sind bei der Stadtpolizei oder bei de Jugenddienschte vom Kanton bereits bekannt zum Teil scho länger also zum Teil chömmet so Usage vo de Jugenddienschtler den chennen wir schon seit feif Johr (.) also quasi sit der Unterstufen oder $\uparrow$. (.) Des isch zum Teil schon no deprimierend au. (.) $\mathrm{S}$ sind so dütliche Hiwies das Gwaltprävention überhaupt nie ne agsetzt hat in dene ihrne Biographie hmhm (.) ja." (Zeilen 88-103)

Herr Unterwasser verweist in ähnlicher Weise wie Frau Blaum auf einen »klaren Auftrag [...] präventiv wirksam zu sein«. Die Aufträge, die ihm tatsäch- 
lich erteilt würden, erfordern jedoch vielmehr Interventionen im Sinne einer möglichst schnellen Lösung des Problems. Die den Aufträgen unterliegenden Probleme seien beispielsweise »schwere Körperverletzung « oder Bedrohung, die eine rasche Reaktion erfordern. An dieser Stelle ließe sich nicht mehr von Prävention sprechen, vielleicht von »Prävention für das nächste Mal«. Die Kluft zwischen dem »übergeordneten« Präventionsauftrag und den alltäglichen Aufträgen hätten Herrn Unterwasser zunächst in ein Dilemma gestürzt, das er inzwischen im Griff hat, indem er Intervention als »frühe Intervention« oder »Prävention« deklariert und gar nicht mehr versucht, es zu trennen. Er deutet seine Tätigkeit als »Prävention für die Zukunft«. Dies begründet er über seine Beobachtung, dass das Thema einen Leidensdruck braucht, bevor die Leute es anpacken. Außerdem kämen die Jugendlichen erst dann zu ihm, wenn es zu spät und bereits erheblicher Schaden in ihrer Biografie angerichtet sei. Damit rekurriert er auf die für ihn bedeutende Fragestellung, was »er einem männlichen Menschen antun müsste (...) damit er dann mit zwanzig ein ausgewachsener Gewalttäter ist«. An diesem Dilemma scheint Herr Unterwasser selbst zu leiden. Seine Deutung von Intervention als Prävention für die Zukunft entwickelt er vor dem Erfahrungshintergrund, dass »Prävention nie angesetzt hat in ihrer Biographie«. Herr Unterwasser kann also weder seinem eigenen Präventionsverständnis noch seinem Präventionsauftrag gerecht werden und muss doch Präventionserfolge aufweisen. Der Bruch zwischen eigenen Präventionsverständnissen, die am Handlungskontext entwickelt werden, und Präventionsaufträgen lässt sich auch in den Darstellungen Frau Schocks beobachten. Die Probleme, die Frau Schock auftragsgemäß lösen soll, beziehen sich auf den Kontext Schule. Diese Probleme soll Frau Schock mit ihrem »magischen Stab lösen. Von diesem Auftrag grenzt sie sich ab, indem sie sagt, an diesen Problemen nicht ansetzen zu können. Sie müsse an den grundlegenden Kommunikationsstrukturen der jeweiligen Familien ansetzen. Dort seien es weniger die Kinder, die Probleme machen bzw. selbst als Probleme anzusehen sind, vielmehr sind sie es, die Problemen ausgesetzt sind. Frau Schock muss sich in dieser Kluft - zwischen der hohen Erwartungshaltung ihrer Auftraggeber und den jeweiligen familiären Herausforderungen - verorten. Dieses grundlegende Dilemma spiegelt sich auch in den folgenden Ausführungen Herrn Tanners bezüglich der aufsuchenden Jugendarbeit wider:

Interviewerin: "Und (.) nochmal zu dem Thema Prävention (.) wie gelingt Prävention (.) was muss dafür da sein dass das gut gelingt?"

Herr Tanner: "Hmhm (.) Anonymität (.) das denke ich ist äh (.) ist etwas Zentrales. (.) Es ist wahrscheinlich auch ein (.) also würde man die Gewalt (.) gesellschaftlich anschauen (.) ist das wahrscheinlich ein Kernproblem der Gesellschaft (.) äh der gesamten Gesellschaft (.) oder oder eine Kehrseite der individualisierten Gesellschaft ist dass äh dass äh häufig und in verschiedenen Feldern einfach da (.) vieles anonymisiert ist. (.) Äh 
ich denke wenn es unseren Jugendarbeiterinnen an der Basis gelingt zu den Jugendlichen die Anonymität aufzulösen Kontakt herzustellen ist schon ganz Vieles geleistet. (.) Äh das gilt sowohl für die Treffs das gilt aber insbesondere auch für die aufsuchende Jugendarbeit (.) äh insofern ist die aufsuchende Jugendarbeit in einem in einem Widerspruch (.) weil sie sagt (.) wir haben keine ordnungspolitischen Auftrag (.) also wir können Jugendlichen nicht zurechtweisen nicht nicht wegweisen und wir wollen das auch nicht weil aufsuchende Jugendarbeit sucht den Kontakt versucht wahrzunehmen was (.) was in der Öff was im öffentlichen raum passiert. (.) Und erst wenn (.) eine Situation wahrgenommen analysiert worden ist und man macht dann man entscheidet sich für eine Intervention (.) dann wird es zu einer Intervention ist aber nicht mehr aufsuchende Jugendarbeit (.) aufsuchende Jugendarbeit nimmt nur wahr und nimmt Kontakte auf (.) und (.) Resultat dieser Arbeit (.) ist aber dass es ruhiger wird im öffentlichen Raum wenn aufsuchende Jugendarbeit geleistet wird und das ist eigentlich ein Widerspruch und wir müssen uns immer dagegen wehren weil es heißt ah aufsuchende Jugendarbeit ihr müsst doch schauen dass Ruhe ist (.) so. (.) Und jetzt warum ist aufsuchende Jugendarbeit denn so erfolgreich in Bezug dass es dann ruhiger wird und ich meine dass es mit der äh mit der mit der Wegfall der Anonymität zu tun hat. (.) Ich glaube auch dass es damit zu tun hat äh (.) Jugendliche ernst zu nehmen $\uparrow$ (.) äh dass sie das Gefühl haben (.) sie werden gehört $\uparrow$ (.) und dass sie zumindest des Gefühl haben (.) ähm was sie sagen (.) ist wichtig (.) beeinflusst etwas (.) und nimmt Einfluss (.) dann wären wir wieder (.) bei der Mitgestaltung oder $\uparrow$ (.) äh das denke sind ich so (.) zwei drei äh k Kerngeschichten (.) die präventiv sehr wichtig sind äh und (.) und etwas weiter und des Weitere ist in der Intervention liegt auch sehr viel Prävention (.) drin und zwar in der Art und Weisen wie man Präventio wie man Intervention macht (.) wegweisen is selten $\uparrow$ (.) das das Richtige es gibt Situation da ist es das einzige Richtige (.) und kann auch Lernschritte ähm." (298-318)

Herr Tanner argumentiert auf die Nachfrage, wie gute Prävention gelingt, zunächst auf einer abstrakten Ebene: Er rekurriert auf seine gesellschaftskritische Positionierung und sieht im Zusammenhang zwischen Gewalt und Anonymität ein zentrales Merkmal »individualisierter Gesellschaften«. Anonymität, erklärt er, sei eine zentrale Ursache für Gewalt. Dieses Argument bedient sich einer historisierenden Deutung von Gewalt, insofern es impliziert, dass es früher anders gewesen sei. An diesem Zusammenhang begründet Herr Tanner die Arbeitsweise offener und aufsuchender Jugendarbeit. Diese haben zum Ziel, Anonymität abzubauen, indem sie einen Kontakt zu Jugendlichen herstellen. Dieses Vorgehen sieht er insofern als Erfolg an, als es »ruhiger wird im öffentlichen Raum«. Dieser Erfolg werde jedoch von der Öffentlichkeit verkannt, die erstens die Erfolge nicht auf die Beziehungsarbeit der Jugendarbeit zurückführt. Zweitens fordere sie von der Jugendarbeit, für Ruhe und Ordnung zu sorgen, was jedoch nicht das eigentliche Ziel der aufsuchenden Jugendarbeit ist. Der Erfolg der Jugendarbeit in dieser Hinsicht ist quasi als 
Nebenprodukt anzusehen. Eine aufsuchende Jugendarbeit, die interveniert, sei keine aufsuchende Jugendarbeit. Diese nehme nur wahr und Kontakt auf. Diesen Schilderungen nach ist Herr Tanner also durchaus an den Erwartungen der Öffentlichkeit orientiert, jedoch nicht hinsichtlich der Arbeitsweise der aufsuchenden Jugendarbeit, sondern hinsichtlich der Bedeutung des Interesses, Ruhe und Ordnung im öffentlichen Raum herzustellen. Seine Normalitätsarbeit ist hier also stark von der Erwartung Anderer vorstrukturiert, obwohl er angibt, seine Arbeit als wirksam und erfolgreich zu erleben. Die zentrale Frage Herrn Unterwassers, was »er einem männlichen Menschen antun müsste [...] damit er dann mit zwanzig ein ausgewachsener Gewalttäter ist« bzw. was »normale Jugendliche zu Monstern mach»«, setzt an einer Kritik struktureller gesellschaftlicher Bedingungen an und ähnelt damit den gesellschaftskritischen Positionierungen, die Herr Tanner und Herr Gold zu Beginn des Interviews einnehmen:

Interviewerin: "((Hustet)) Und können Sie ähm beschreiben wenn Sie so an Ihre alltägliche Arbeit denken welche Formen von Gewalt oder jugendlicher Gewalt (.) ähm (.) gibt es oder mit was haben Sie zu tun was sind das für-«

Herr Unterwasser: "Ja ich chann nur sagen mit was ich z tue hän. Ich stune immer wieder was es sonscht au no gibt oder. $\uparrow$ Also des isch schon o so. (.) Ich bin aufm Land ufgwachse in ner $m$ Gebirgskanton ((schluckt)). Da gibts da ganz viel Phänomen die gibt's da au schon lang die han ich nöd chennt. (.) Ja also eis Phänomen zum Bispiel für ebbes wo ich nöd chennt is Hügle. (.) Hügle isch (.) wenn des machet vor allem Jungs (.) ähm (.) wenn öbbert (.) quasi ((lacht)) zuunderscht liegt und die ganz Bezugsgruppe ober druf als Hügel als des wort Hügle chummt von Hügel mir machet $n$ Hügel (.) und $s$ gibt wie verschiedene Forme von Hügel also es gibt wirklich es es ähm demüetigends es es es Trauma wirklich es traumatisches Hügle also das cha bis zu Erstickigsängscht gah (.) wo man öbbert wirklich chann plage mit dem Hügle und es gibt des fründschaftlichs Hügle will es isch halt au luschtvoll es gibt viel Riebig oder $\uparrow$ viel Chörperkontakt das erläbet d Jungs durchus als als luschtvoll und des is nöd schwul. (.) So ((lacht)) die Gfahr bestaht bestaht da nöd (.) also es isch ebbes wos als Phänomen gibt und des wird nöd in Frag stellt (.) und das isch so ebbes isches ebbes gsi won ich woin ich das erschte mal chenneglernt bin ich verschrocke will ich gfunde hab das is ja wahnsinnig der wo zuunterscht liegt ebbe der der ringt um sis Läben oder $\uparrow$ (.) und das passieret überall in die Schuelhüser (.) und und erscht in der Useinandersetzung (.) ja das das chann halt au cool si und luschtvoll und lässig und und und da isch so wichtig das d Lehrer überprüfet wasch isches für a Art von Hügle das isch eis Bispiel für Ebbes wo n ich näd chennt hab. (.) Dann bin ich konfroniert mit Mobbing (.) i der verschiedenschte Form in der verschiedenschte Ebbene det find ich d Maidli sehr hüfig sehr en aktive Rolle ((atmet tief ein)) Ja (3.0) denn isches so die die die ganz normale banale Gwalt die Tätlichkeite in allen Forme ich sig das vo vo (2.0) vo Raubüberfäll Erpressigsversüech Racheäkt (2.0) Bande (.) oder reschpektiv so Gruppe Grupperivalitäte Skater gege 
Hiphopper so die Gschichte. (3.0) Für mich isch halt (3.0) grundsätzlich ich chann ich chann (.) relativ viel Witerbildige bsucht zum Bereich Gwalt zum Teil Workshops wos drum gangen isch selber au gwalttätig $z$ werde also zum Bispiel so Impact Kürs wo wos drum gaht wie isch des wenn ich öbberen ins Gsicht ginke und da gibt's n Gink miter em Schongummihelm und ich muess dem jetzt ins Gsicht ginke und weiß dann aber au wie sich des agfühlt hätt. (.) Chann halt die Erfahrig mache dös isch luschtvoll $\uparrow$ (.) es is $n$ absoluter Machtkick wenn ich öbbert wenn ich öbbert so wit verprügle chann bis der sich nümmer bewegt ((holt luft)) und dem a no ins Gsicht ginke wenn der schon am Bode liegt des isch nöd eifach nur chrank und morbid sondern da das das isch n Macht (1.5) $\mathrm{n}$ Machtüberdorsis die die isch wirklich beruschend und das ist nachvollziehbar ((holt luft)). Vo det her (.) all die Sache die (.) die find ich mittlerwile relativ normal ((lacht)) oder relativ (.) nöd normal nöd nöd dass sie mich falsch verstehn aber aber ich finds nöd (.) es brucht nöd all zu sehr d Suche nach was macht (2.5) was macht die normale Jugendliche zu dem Monschter oder das sind nöd Monschter das sind ganz normale Jugendliche wo so Sachen machet die sind ähm (.) das is durchus verständlich verstehbar und nachvollziehbar wie die det hiechömmet wenn ma das selber erläbt hat (.) und da gibt's halt wirklich eifach alle Forme von Grenzüberschritige (3.0) und da find ich halt a grundsätzlich isch dös nöd primär nur männlich ich find schon oder bi der bi der Jungs und Manne isches speziells Thema wil (1.5) wil die Geschlechtsidentität fescht mit aggressiven Verhalte verbunde isch also ich bin es attracktivs Männli (.) wenn ich quasi chann Rivale verjage ich find die Ebene spielt halt wirklich immer no a Rolle. (.) Aber also zum Bispiel mini Schwöster ich hän en ältere Schwöster die hätt äh die isch Krankenschwöschter gsi ( ) die hätt bi ihrer Diplomarbeit als Krankenschwöschter d Arbeit gschriebe über Säuglingsschwöschtere wo Säugling misshandlet (.) und sie hat so die ganze Palette von mögliche Misshandligstechniken und Strategie vorgstellt wo so agwendet werdet bei de Säuglingsschwöschtere. (.) Des isch wirklich des des liest sich wie nes Protokoll vo vo de vo de Verhörmethode vom $f$ vo vo ich weiß au nöd wer wer da so krass verhört isch dös FBI oder CIA CIA. (.) Es liest sich ganz ähnlich oder $\uparrow$. Es sind perfideschte Foltermethode und des sind die liebe guete Fraue und Krankenschwöschtere wo des machet und natürlich als (.) Opfer von der Überforderig. (2.5) Oder $\uparrow$ also mir händ ja de (.) wenn a Frau Gwalt awendet denn isch des Expressiv als Usdruck für Überforderig und beim Ma isch es böse Absicht. (.) Ich find mit dem han ich sehr Mühe. (.) Es git es git Grausamkeite und Grenzüberschritige bi beiden Gschlechter. (.) Bi de Jungs isch sie eifach (1.5) stärker im Vordergrund weils vo de Jungs zum Teil au erwartet wird. (4.5) Es ghört (.) es ghört zum zum Mehrwert au vom Gschlechterwert das Jungs sich a äh sich aggressiv gebaret in gwüssen Situationen (.) es wird von inne verlangt. (3.5) Ja und und (1.5) da bin ich mit ziemlich allem konfroniert ebbe vo vo Hänseleie vo Sachbeschädigung bis hi zu wirklich schwäre Körperveletzig bis hi zum Todschlag (.) ja (.) da gibts die ganze Bandbreite." (457-498)

Herr Unterwasser unterscheidet auf die Frage nach den Formen von Gewalt bzw. Jugendgewalt verschiedene Formen von Gewalt bzw. Jugendgewalt. Dabei 
sind zunächst v.a. geschlechterspezifische Unterscheidungen relevant. Für gewisse Handlungsformen bringt er sehr viel Verständnis auf, und zwar sowohl aus seiner eigenen Erfahrung mit Gewalt als auch aus gesellschaftskritischer Perspektive heraus. Gewisse, »männliche « Gewaltformen, etwa das »Hügeln«, sieht er als »normal« an. Zwar habe ihn das »Hügeln« zunächst erschrocken, in der Auseinandersetzung damit habe sich seine Perspektive jedoch verändert. Hügeln könne »lustvoll«, »ässig« und »cool« sein. Eine ähnliche Erfahrung habe er auch im Rahmen eines Workshops gemacht, in dem es darum ging, selbst gewalttätig zu werden. Auch dies sei eine »lustvolle« Machterfahrung. Damit vollzieht Herr Unterwasser einen Perspektivenwechsel bzw. eine Perspektivenübernahme: Er versetzt sich in den Täter hinein, um dessen Handeln zu verstehen. Im Unterschied zu den meisten der anderen befragten Fachpersonen normalisiert er Jugendgewalt demnach nicht (nur) über Biologisierung, Naturalisierung, Historisierung oder Kulturalisierung, sondern anhand seiner eigenen (erlernten) Gewalterfahrung. Sein Verständnis für »männliche Täter« geht noch weiter: Er sieht »Männer« als »Opfer« von Sozialisationsprozessen, die er während des Interviews wiederholt als »feministisch « bezeichnet und die u.a. daraus resultieren, dass die Institutionen der Erziehung und Bildung von Frauen dominiert sind. Von Buben bzw. Männern werde ein gewisses Maß an Aggressivität erwartet. Damit sei Gewalt an männliche Identität im Sinne normativer Erwartungen geknüpft. Daraus entwickelt Herr Unterwasser letztlich ein besonderes Schutzbedürfnis von Buben. »Weibliche« Gewalt, so kritisiert er, werde von der Gesellschaft, die eben feministisch geprägt sei, gerechtfertigt, indem sie diese beispielsweise als Umgang mit Überforderung deutet. Einer gesellschaftlichen Skandalisierung »männlicher« Gewalt, mit der eine Akzeptanz für »weibliche« Gewalt einhergeht, setzt er eine Akzeptanz für »männliche« Gewalt und eine Skandalisierung »weiblicher« Gewalt gegenüber. Damit beschuldigt er Frauen, vorsätzlich zu handeln, und setzt sie mit Folterern gleich. Die wahren Täter sind aus seiner Perspektive Täterinnen. Damit konstruiert er eine Täterperspektive auf Frauen in Abgrenzung zu einer Opferperspektive auf Männer, die er bereits in der einleitenden Sequenz eingeführt hat. Dort entwickelt er eine Opferperspektive auf männliche Gewalttäter. Seine »Normalisierungsarbeit« bezieht sich also auf die Normalisierung von Männergewalt in Abgrenzung zu Frauengewalt. Diese Konstruktion beeinflusst seine Arbeit. Er gibt an, ausschließlich mit Buben zu arbeiten, zumindest was Prävention an Schulen betrifft. Insofern geht mit dieser Normalisierungsstrategie auch die Konstruktion einer besonderen Hilfsbedürftigkeit von Buben einher. Die für ihn zentrale Frage danach, was »er einem männlichen Menschen antun müsste [...] damit er dann mit zwanzig ein ausgewachsener Gewalttäter ist« bzw. was »normale Jugendliche zu Monstern macht«, entwickelt er also vor dem Hintergrund einer Gesellschaftskritik, insbesondere einer Kritik des, aus seiner Perspektive, machtvollen Feminismus. Buben 
seien in diesem Zusammenhang als besonders schutzbedürftig anzusehen. Welche pädagogischen Maßnahmen leitet er neben der Konzentration auf Jungenarbeit daraus ab? Wie er am Beispiel des »Hügelns « angibt, hätten die Lehrer die Aufgabe, dies zu überprüfen. »Kontrolliertes« Hügeln hält Herr Unterwasser somit also nicht nur für tolerierbar, sondern auch für förderlich. Jedoch gibt es »alle Formen von Grenzüberschreitungen«, auf die die Interviewerin zu sprechen kommt.

Interviewerin: "Und Sie haben jetzt auch gesagt dieses (.) Hügle zum Beispiel (.) ähm (.) des gibt's in Ausprägungen die halt auch akzeptiert werden können?»

Herr Unterwasser: "Es gibt n positivs Hügle genau."

Interviewerin: "Wo sind denn da die Grenzen zwischen Gewal- also zwischen soner akzeptierbaren Gewalt und einer Gewalt die halt-«

Herr Unterwasser: »Hmhm dös ist jetzt für mi öbbes und das empfählet mir den Schulen au (.) solchene Spielrüm offe zu äh uf uf $z$ tue also wie sie z erlaube es gibt positive Forme vo Rangeleie einzelne Schuelhüser richten das ganz bewusst i. (.) I dem Ecke dürfet ihr chämpfe (.) unter der Vorussetzig dass es faire Chämpf sind (.) dass heißt man muss fröge man muss es vorher es Beziehigs a Beziehigsafrag mache a Beziehigsagebot ich möchte mit Dir chämpfe bischt Du bereit dazu? (.) Das muss klärt si und ma muss sich verspreche ich chämpfe fair. (.) Denn dörf ma det $\uparrow$ (.) i dem Ecke vom Schulhusareal dürf mag 0 chämpfe (.) und dann chann ma sich au druf verla dass nöd ständig Erwachsene chömmet go störe. (.) Ab und zu wird au kontrolliert ob au wirklich sich alle an $\mathrm{d}$ Regle haltet und (.) mit der Uflag sich entweder bei der Peacemaker oder bei der Lehrerin je nachdem was ma da inschtalliert hät (.) z melde wenns ebbe nöd super lauft (.) und zum Bispiel Hügle (.) da is eusere chlare Empfehlig an d Lehrer lönt das zue als kläret ab isch a positive Form von Hügle also das heißt der wo $z$ unterscht liegt muess ma fröge isch des für sich ok (.) und wenn der sait desch is ok und ich han ber $\mathrm{s} G$ fühl der sait desch is ok damit er nöd vo der Gruppe no mehr sanktioniert wird (.) denn unbeidingt interveniere (.) alle außernand neh und wirklich (.) wirklich stelle konfrontiere mit (.) ich glaub eu nöd (.) ich hans Gfühl dasch is für inn gar nöd luschtig (.) und jetzt will ich wüsse was da lauft wie chummt das warum liegt der da $z$ unterscht und wird $g$ hüglet. (2.5) Und wenn man aber wirklich s Gfühl hät wohl das alle am juchzen und geuße und sich rieben und tue und mache (.) durchus au sage cool (.) lässig (.) soll i a noch druf liege oder $\uparrow$. Also es is wirklich es schafft halt Nähe und Vertrauen und ja ja. (2.0) Genau (.) und isch wichtig fürs sozials Lerne find ich also das ghört is wie $\mathrm{n}$ Teil dazue oder $\uparrow$ weil sonscht wird's dann wirklich steril.“ (Zeilen 499-518)

Auf die Frage nach der Grenze der Akzeptanz des »Hügelns « positioniert sich Herr Unterwasser als Fachperson für den schulischen Kontext. Er empfehle den Schulen, Räume für »Rangeleien«, »Kämpfe« bzw. »faire Kämpfe« bereitzustellen, diese aber zu kontrollieren: »Hügeln« sollte bestimmten Spielregeln unterliegen, welche die dabei ausgeübte Gewalt normalisieren, z.B. Fairness 
oder die Abstimmung darüber, wer mit wem kämpft. Die »Kämpfe« seien zu beobachten und im Zweifel zu hinterfragen oder es müsse interveniert werden. In dieser Hinsicht entwickelt Herr Unterwasser, in ähnlicher Weise wie Herr Tanner und Frau Blaum, ein Präventionsverständnis, das darauf abzielt, den Schülern zu ermöglichen, ihre Grenzen auszutesten, Lern- und Bildungserfahrungen zu machen, insbesondere in Bezug auf das Erlernen von Regeln und Artikulationsformen. Dies sei jedoch nur in einem bestimmten Rahmen möglich, der durch Erwachsene kontrolliert wird. So sei »normales«, »akzeptables« Gewalthandeln - eine »positive Form von Hügeln « - möglich. Auch in diesem Kontext kann Prävention als normativ angesehen werden, insofern als sie darauf abzielt, dass »normale« Jugendgewalt »normal« bleibt. Jugendgewalt bzw. die Normalisierung dieser wird damit zu einem pädagogischen Konzept, das an Schüler (re-)adressiert wird - das »Hügeln « wird gewissermaßen überformt: Ursprünglich kein pädagogisches Konzept, sondern Schulpausenspiel von Buben, findet es Eingang in die pädagogische Praxis, speziell im Kontext von Schule und Bubenarbeit. Für Herrn Unterwasser ist die Pädagogisierung des »Hügelns«

"wirklich positivs Bispiel (1.0) für buebespezifische (.) Gschichte im Bereich Gwaltprävention fallt mir wirklich die Champfesspiele fallet mir i und sonscht nimmer so wahnsinnig viel. (2.0) Grundsätzlich viellicht ganz generell so ( ) erläbnispädagogische Gschichte (.) scho oder (.) das scho (.) also ich finde mit Buebe ischs liechter übers tue übers miteinand mache z schaffe (.) als als irgendwo im äh Schuelzimmer im Kreis z sitzen und das uf der Gsprächsebene auf der intellektualisierten Ebene wella bearbeite das isch mit ((holt luft)) mit Jugendlichen und und und und Bueben im Chinderalter eifach nöd so möglich (.) oder nöd so ergiebig (....).“ (Zeilen 304-311)

Seine Beobachtung, dass Gewalthandeln von Buben in einem Zusammenhang mit Erwartungen an ihre Geschlechtsidentität bzw. der Entwicklung dieser steht, erfordert bubenspezifische Präventionsarbeit, in der Buben lernen, sich zu disziplinieren und eine akzeptable Form von Gewalt auszuüben. Auf Gesprächsebene könnten Jugendliche und v.a. »Buben im Kinderalter« nicht abgeholt werden. Diese Argumentation unterstreicht neben einer Normalisierung von Jugendgewalt wiederum die defizitorientierte Perspektive auf Kinder und Jugendliche, mit der pädagogische Praxis gerechtfertigt wird. Die Kinder und Jugendlichen, die »hügeln«, hätten von sich aus nicht die Kompetenzen, Regeln einzuführen, einzuhalten und rechtzeitig zu stoppen. Es benötige einen Rahmen, in dem Erwachsene dies kontrollieren können. Damit geht das Präventionskonzept, das auf Bildungs- und Kompetenzerwerb abzielt, gleichzeitig mit einem Kontrollauftrag einher und kann damit als eine Form von Disziplinierung angesehen werden, die durch Re-Adressierung verstärkt wird. 
Dies lässt sich hier sowohl anhand schulischer Kontexte als auch anhand der offenen und aufsuchenden Jugendarbeit nachvollziehen:

Herr Tanner: "Ich ich seh da ich dass ich mir da noch etwas aufgeschrieben habe und das geb ich so in einer Frage was denn unsere Aufgabe such ist ähm und und auch in der Prävention und zwar in der Frage nach der Aggression (.) und das die halt eben dazugehört. (2.0) Und sie ist negativ besetzt aber eigentlich ist sie ist sie wie ab ich behaupt jetzt mal den Urspung dass wir überhaupt überleben (.) äh äh ist die Aggression ein wichtiger Bestandteil und ((räuspert sich)) es gehört gehört wie zu unserer Aufgabe in der Jugendarbeit zu schauen (.) dass das man Lernfelder (.) bietet (.) wo die Aggression gelernt werden kann (.) das kann eine Kraftmaschine sein die wir in einem in einem Jugendtreff haben das kann aber auch Tanzbewegung (.) äh das kann das können all die Sitzungen im im und Aushandlungsprozess in Projekten sein (.) äh Lernfelder wo die Jugendlichen lernen können (.) mit inrer Aggression umzugehen (.) auszuleben (.) diese auch zu artikulieren so. " (Zeilen 415-422)

Herr Tanner hat sich offensichtlich auf das Interview vorbereitet, indem er sich vorher etwas aufgeschrieben hat, an dem er sich orientiert. Einer dieser wichtigen Punkte betrifft die Frage nach Aggression. Er sehe es als zentrale Aufgabe der Jugendarbeit an, den Jugendlichen »Lernfelder « zu bieten, in denen sie Aggression lernen können. Diese Perspektive verdeutlicht die Normalisierung von Jugendgewalt und daran anschließend die (Be-)Deutung eines Präventionskonzepts, das darauf abzielt, Normalität aufrechtzuerhalten. In diesem Sinne ist Prävention als eine Art von Disziplinierung zu verstehen, die darauf hinarbeitet, dass Kinder und Jugendliche den normativen Erwartungen der Gesellschaft entsprechen. Die dahinterliegende gesellschaftskritische Positionierung dient dabei der Rechtfertigung dafür, Jugendliche in Schutz zu nehmen und sie gleichzeitig unter Kontrolle zu stellen, und damit der Legitimation der eigenen Fachbereiche, die so gesehen von einem Diskurs über Jugendgewalt, der u.a. skandalisiert, genährt werden.

Aus den Interpretationen geht ein den Fachpersonen weitgehend gemeinsames Orientierungsmuster in Bezug auf Jugendgewaltprävention hervor. Ihr Präventionsverständnis orientiert sich an einer Normalisierung von Gewalt in einem doppelten Sinne: Das Gewalthandeln Jugendlicher wird anhand biologisierender und kulturalisierender Zuschreibungen sowie historisierender Muster gedeutet. Diese Deutungen werden an der eigenen Biografie, der Wissenschaft sowie an Situationen der Gewalt validiert und damit reproduziert. Das den Fachpersonen eigene Präventionskonzept zielt im Anschluss an diese Deutungen auf eine Normalisierung von Jugendgewalt in dem Sinne, dass es auf eine Normalisierung individueller Lebensverläufe abzielt und gewisse, auf Gewalt bezogene Handlungsformen legitimiert. Das Präventionsverständnis 
der Fachpersonen bricht insofern mit konkreten Präventionsaufträgen, als diese an Evidenz orientiert sind: Erfolge sollen schnell sichtbar und nachhaltig sein. Dies widerspricht jenem Präventionskonzept, das an individuellen Lernund Bildungsbiografien ansetzt und nicht messbar ist bzw. unter Umständen erst retrospektiv am individuellen Fall erfasst werden kann. Die Fachpersonen zeigen vor dem Hintergrund von Präventionsaufträgen, dass sie diesen durchaus nachgehen, indem sie beispielsweise individuelle Erfolge medial inszenieren oder Erfolge in Bezug auf ihre Interventionsarbeit als präventive Erfolge ausgeben. Die befragten Fachpersonen entwickeln und normalisieren eine defizitorientierte Perspektive auf Kinder und Jugendliche, über die sie ihr Präventionskonzept rechtfertigen, was als Legitimationsstrategie in Bezug auf eine Distanzierung gegenüber Präventionsaufträgen gedeutet wird. Im folgenden Kapitel werden die zentralen Ergebnisse der Interpretationen zusammengefasst sowie auf methodische Aspekte eingegangen.

\subsubsection{Zusammenfassung}

Die befragten Fachpersonen entwickeln ihre Expertisen aus einer defizitorientierten Perspektive auf Jugendliche heraus, wobei sie deren Defizite nicht auf elterliche Erziehungspraktiken zurückführen (vgl. Dollinger/Schmidt-Semisch 2011: 14), sondern auf ein grundsätzliches hegemoniales Verhältnis zwischen Natur und Kultur, insoweit sie Jugendgewalt biologisieren, naturalisieren, kulturalisieren, historisieren und damit normalisieren. Sie entwickeln ihre defizitorientierte Perspektive aus einer Gesellschaftskritik heraus. Dies wird v.a. an den Interviews mit Herrn Tanner und Herrn Gold deutlich, deren gesellschaftskritische Positionierung zu Beginn der Interviews eine enorme Präsenz einnimmt, emotional bestärkt wird und als Distanzierung von einer von außen zugeschrieben Expertenrolle gedeutet werden kann. Die Adressierungsweisen der Interviewerin, die Zuschreibungen hinsichtlich einer problemorientierten Betrachtungsweise von Jugendgewalt sowie "guter« Jugendgewaltprävention enthalten, aktivieren gesellschaftskritische Positionierungen, nach denen Jugendliche aus biologischen, medizinischen und kulturellen Gründen »leichte Opfer« von gesellschaftlichen Diskriminierungsprozessen und daher in Schutz zu nehmen seien. Die Kompetenzen, insbesondere sprachlicher Art, sich dagegen zu wehren, werden den Jugendlichen abgesprochen. Auch Bildungsdefizite ließen den Jugendlichen nicht die Fähigkeit, sich angemessen zu artikulieren. Die Fachpersonen gebrauchen gesellschaftskritische Positionierungen, um ein Bedrohungsszenario zu konstruieren, vor dessen Hintergrund sie das besondere Schutzbedürfnis Jugendlicher entwickeln. Daran entwickeln sie auch ihre eigenen Präventionskonzepte: Sie sehen ihre Aufgabe insbesondere darin, Jugendliche dabei zu unterstützen, Lern- und Bildungserfahrungen zu machen, was jedoch innerhalb eines kontrollierbaren 
Rahmens erfolgen müsse. Diese Aufgabe resultiert also aus der Annahme eines Schutzbedürfnisses Jugendlicher, die mit einer defizitorientierten Perspektive einhergeht. Dies impliziert die Annahme, dass Gesellschaft nicht veränderbar ist bzw. nicht verändert werden soll. Damit reproduzieren die Fachpersonen einen Dualismus zwischen der Gesellschaft und den Jugendlichen, der sich in den Deutungsmustern von Jugendgewalt widerspiegelt: Hier wird zwischen »normaler« und »pathologischer« Jugendgewalt differenziert. Die Fachpersonen beziehen ihre eigenen Präventionsverständnisse auf eine Normalisierung von Jugendgewalt im doppelten Sinne: Zum einen ist für sie eine Unterscheidung zwischen »normalem« und »pathologischem« Gewalthandeln grundlegend in Bezug auf eine Entscheidung für oder gegen Intervention bzw. Prävention. Intervention und Prävention sind in diesem Zusammenhang als zwei Seiten einer Medaille anzusehen. Zum anderen zielen ihre Präventionskonzepte auf eine Normalisierung individueller Lebensverläufe ab. Die Deutung von Jugendgewalt und die sich daran anschließenden Entscheidungen für oder gegen Intervention sind am konkreten Fall oft nicht oder nur schwer möglich, wodurch die Interventions- und Präventionskonzepte verschwimmen. Deutlich wird jedoch, insbesondere an der aufsuchenden Jugendarbeit, dass sich die Erwartungen der Öffentlichkeit oder anderer Institutionen in Bezug auf die Frage, wann und wo interveniert werden soll, deutlich von den Entscheidungen der Jugendarbeiterinnen und Jugendarbeiter unterscheiden und es dabei zu Konflikten kommen kann. Andere Fachpersonen, etwa Frau Schock, Herr Gold oder Herr Unterwasser, haben hingegen mehrheitlich mit Fällen zu tun, bei denen bereits interveniert worden ist. Dort können eigene Verständnisse von Prävention weniger gut greifen, da die diesen zugrunde liegenden Problematisierungsweisen nicht mehr Grundlage für eine Entscheidung sind. Gemeinsam ist den Befragten, dass sie nicht Prävention, sondern Intervention als primäre Aufgabe ihrer jeweiligen Fachbereiche ansehen. Intervention ist ein notwendiges Konzept, da Grenzüberschreitungen der Jugendlichen alltäglich sind, insbesondere in der offenen Jugendarbeit. Dabei grenzen sie zwei miteinander konkurrierende Präventionsverständnisse voneinander ab: Ein Präventionsverständnis, das in seiner Außenorientierung auf Wirksamkeit, Evidenz und Nachhaltigkeit abzielt und v.a. in Form konkreter Aufträge adressiert wird, steht einem Präventionsverständnis gegenüber, das auf Ermöglichung und Unterstützung von Lern- und Bildungsprozessen sowie individueller Lebensgestaltung abzielt und aus den jeweiligen Handlungskontexten heraus entwickelt wird. Diesem Präventionsverständnis nach wird Prävention jedoch in den Verantwortungsbereich der Jugendlichen selbst gestellt: Frau Blaum etwa »entlässt« den Jugendlichen mit einem konkreten Bildungsauftrag. Herr Unterwasser lässt die Schüler miteinander kämpfen, damit sie die Grenzen des Akzeptablen am eigenen Leib erfahren. So gesehen haben die Jugendlichen für Erfolge in Bezug auf Kompetenzerwerb und Bildung oder gar 
für die Realisierung einer »erfolgreichen Lebensgestaltung« selbst Sorge zu tragen, was nur individuell und retrospektiv messbar ist. Dieses Präventionsverständnis schließt gleichzeitig Kontrolle mit ein. In dieser Hinsicht lässt sich sagen, dass sich die befragten Fachpersonen in ihren »eigenen « Präventionsverständnissen von konkreten Präventionsaufträgen insofern abgrenzen, als sie ihre Präventionskonzepte nicht an einer auf Wirksamkeit, Evidenz und Nachhaltigkeit basierten Prävention orientieren. Die Fachpersonen können zwar die Rahmenbedingungen für Lern- und Bildungsprozesse bereitstellen, Prävention aber nicht nachhaltig verfolgen oder gar messen. Eigene Verständnisse von Intervention und Prävention beziehen sich auf das »Innen« der jeweiligen institutionellen und organisationsspezifischen Kontexte und basieren auf je spezifischen Problematisierungs-und Deutungsweisen. Die Erzählung von Frau Blaum zeigt jedoch, dass die Rekonstruktion von Wissen insofern stigmatisierend sein kann, als sie dieses auf individuelle Merkmale bzw. konkrete Situationen projiziert und damit Risiken individualisiert. Die defizitorientierte Perspektive, das damit verbundene Schutzbedürfnis Jugendlicher und die damit verbundene Präventionsarbeit beziehen sich nicht in erster Linie auf Individuen, sondern auf eine soziale Gruppe und forcieren damit Risiken. Eine der zentralen Thesen der hier vorliegenden Arbeit ist demnach, dass über die Normalisierung von Jugendgewalt Risiken insofern individualisiert werden, als die Normalisierung von Jugendgewalt im Sinne jugendlichen Gewalthandelns verallgemeinert wird. Die Außenorientierung irritiert dabei die Innenorientierung, begünstigt damit die Individualisierung von Risiken und somit Stigmatisierungsprozesse. In diesem Zusammenhang kann die defizitorientierte Perspektive auf Jugendliche als eine Grundhaltung der hier befragten Fachpersonen angesehen werden und der Präventionsdiskurs als Risikodiskurs gelesen werden. Hinsichtlich der Forschungsfrage, inwieweit sich die Fachpersonen im Kontext von Jugendgewaltprävention an kriminalpolitischer Programmatik orientieren, lassen die Interpretationen zusammenfassend darauf schließen, dass die befragten Fachpersonen bezüglich ihrer Problematisierungsweisen und daran entwickelten Präventionsverständnisse in einem ambivalenten Verhältnis zu kriminalpolitischer Programmatik stehen, das auch durch die Adressierungsweisen der Forscherin vermittelt wird. Dies wird zunächst an verschiedenen Formen der Distanzierung gegenüber der durch die Interviewerin eingebrachten Zuschreibungen deutlich, insbesondere an den daran entwickelten gesellschaftskritischen Positionierungen, vor deren Hintergrund die Befragten ihre Expertisen konstruieren: Sie konstruieren ein Schutzbedürfnis Jugendlicher, an dem sie in ihren Konzepten anknüpfen. Dieses Schutzbedürfnis resultiert aus einer defizitorientierten Perspektive, aus der heraus sich Jugendliche aufgrund mangelnder Artikulationsfähigkeiten und Kompetenz und aufgrund von Bildungsdefiziten nicht gegen gesellschaftliche Diskriminierungsprozesse und strukturelle Ungleichheiten zur Wehr 
setzen könnten. In dieser Hinsicht zielen die gesellschaftskritischen Positionierungen und die damit einhergehenden Handlungs- und Begründungsweisen nicht auf grundlegende Veränderungen der Gesellschaft, sondern auf die Reproduktion des eigenen professionellen Selbstverständnisses und die Legitimation der eigenen »Profession«. Die den Fachpersonen eigenen Präventionsverständnisse zielen darauf ab, Rahmenbedingungen zu schaffen, die es Jugendlichen ermöglichen, Anpassungsprozesse im Sinne einer individuellen Entwicklung und Lebensgestaltung zu vollziehen. Mit ihren konkreten Problematisierungsweisen und sich daran anschließenden Verständnissen von Intervention und Prävention orientieren sich die Befragten also nicht primär an kriminalpolitischer Programmatik im Sinne einer evidenzbasierten, nachhaltigen und messbaren Prävention, die sie aufgrund der Zugzwänge, Restriktionen und Möglichkeiten ihrer je spezifischen Tätigkeitsbereiche nicht verfolgen können, sondern an ihren jeweiligen professionellen Handlungskontexten, die jedoch durch Normalitätserwartungen strukturiert sind. Herr Tanner und Frau Blaum sind im Rahmen ihrer Tätigkeit in der offenen Jugendarbeit mit der alltäglichen Präsenz »normalen« jugendlichen Gewalthandelns konfrontiert und können unter dieser Bedingung Prävention nur rahmen. Die offene Jugendarbeit kann also als »Interventionsfeld « angesehen werden, in denen das Jugendlichen durch Lernerfahrungen Möglichkeiten für Prävention, verstanden als Bildungs- und Kompetenzerwerb, eröffnet, in dem die Jugendlichen aber auch kontrolliert werden können. Prävention geht jedoch letztlich über den Verantwortungsbereich der offenen Jugendarbeit hinaus, insofern sie an Jugendliche in Form von Aufträgen re-adressiert wird und auch andere Institutionen problematisiert werden. Gleichzeitig sehen sich beispielsweise Herr Tanner und Frau Blaum jedoch selbst mit einem »Präventionsauftrag « konfrontiert, den sie nicht einlösen können, da dies das basale Vertrauensverhältnis gefährden würde, das ihre Arbeit mit Jugendlichen voraussetzt. Prävention, die nur retrospektiv am individuellen Fall beurteilt werden kann, steht somit Prävention als messbares und sichtbares Konstrukt, wie es von außen vermittelt wird, gegenüber. In dieser Hinsicht kann die Entwicklung der eigenen Expertise aus einer gesellschaftskritischen Positionierung heraus als Abgrenzung zu kriminalpolitischer Programmatik gedeutet werden. Bemerkenswert ist, dass Frau Blaum - trotz dieser Distanzierung in ihrer Erzählung - Risiken in die Situation projiziert, womit sie Risiken individualisiert und den Jugendlichen damit stigmatisiert. Dies lässt sich dahingehend deuten, dass sie ihr eigenes professionelles Selbstverständnis in der Orientierung an kriminalpolitischer Programmatik infrage stellt. Diese Deutung wird durch die Beobachtung bestärkt, dass Frau Blaum in der Orientierung an statistisch generiertem Wissen an ihrem eigenen Erfahrungswissen zweifelt. So machen die Interpretationen, insbesondere die Erzählung Frau Blaums, auf zwei Prozesse aufmerksam, die bezüglich der Forschungsfrage theoretisch relevant sind. 
Zum einen stellt sich die Frage, welche Konsequenzen auf der Ebene der jeweiligen Fachbereiche aus der Orientierung an kriminalpolitischer Programmatik resultieren? Zum anderen stellt sich die Frage, welche Konsequenzen dabei für die Adressatinnen und Adressaten der jeweiligen Fachbereiche, etwa der Jugendlichen, denkbar sind? So kann erstens angenommen werden, dass eine verstärkte Orientierung der Fachpersonen im Kontext von Jugendgewaltprävention an kriminalpolitischer Programmatik Verunsicherungen der eigenen Expertise begünstigt. Zweitens kann angenommen werden, dass eine Orientierung an individuellen Risiken Stigmatisierungsprozesse begünstigt. Der Jugendliche, von dem Frau Blaum erzählt, verschwindet nicht nur aus der offenen Jugendarbeit, er verschwindet bereits in der Erzählung Frau Blaums, in der sie ihn weniger als Person mit individuellen Bedürfnissen darstellt, sondern vielmehr bestimmte Risiken auf ihn projiziert; insofern individualisiert sie ihre Defizitperspektive. An der vergleichenden Interpretation der Interviewsequenzen, die Prävention in Bezug zu Devianzkonstruktionen setzt, lässt sich zusammenfassend festhalten, dass die Fachpersonen ihre je eigenen Präventionsverständnisse aus den jeweiligen Kontexten sozialer Praxis heraus entwickeln. Dieser Blick auf die eigenen Praktiken und darin angesiedelten Möglichkeiten und Grenzen von Prävention sind jedoch geprägt von einer normalisierenden Sichtweise auf Jugendgewalt sowie von der Erfahrung, dass »normale« Jugendgewalt alltäglicher Bestandteil der Arbeit ist und somit primär Intervention erfordert. Jugendgewalt wird normalisiert im Rekurs auf entwicklungspsychologische, biologische und kulturelle Annahmen sowie auf eigene biografische Erfahrungen. Diesbezügliche Formen von Jugendgewalt bzw. jugendlichen Gewalthandelns werden abgegrenzt von »schlimmen« Gewaltformen oder Gewaltformen, die sich erst im Erwachsenenalter zeigen und pathologisiert werden. Im Anschluss an diese normalisierenden Deutungen von Jugendgewalt schließen Präventionsverständnisse an, die darauf abzielen, Räume zu ermöglichen, in denen Jugendliche sich eigenständig oder gemeinsam mit den Peers bilden, Kompetenzen erwerben, lernen, ihre Grenzen austesten und auch Fehler machen können, ohne dafür sanktioniert zu werden. Die Fachpersonen schreiben sich selbst dabei eine unterstützende und gleichzeitig kontrollierende Rolle zu. Der Möglichkeitsraum kann aber gleichzeitig als Raum verstanden werden, in dem bestimmte normative Erwartungen vermittelt werden (bezüglich der »richtigen« Einstellung zum Koran oder bezüglich dessen, was ein erfolgreiches Leben ausmacht). Letztlich fordern die Fachpersonen trotz ihrer gesellschaftskritischen Positionierungen Jugendliche dazu auf, sich den gesellschaftlichen Gegebenheiten anzupassen. Erfolgreiches Leben bedeutet aus deren Perspektive nicht, gegen gesellschaftliche Ungleichheiten und Ungerechtigkeiten aufzubegehren, sondern sich damit zu arrangieren - »sich seinen Platz zu ergattern in dieser Gesellschaft«, wie es Herr Schäfer formuliert. Letztlich sind die Jugendlichen in der Realisierung 
dieser normativen Erwartungen auf sich selbst gestellt und tragen dafür die Verantwortung. Dieses Präventionsverständnis hat nicht den Anspruch, Jugendgewalt zu verhindern (was auch nicht als realistisch eingeschätzt wird), sondern das »normale« Maß an akzeptierbarer Jugendgewalt aufrechtzuerhalten. Dabei stehen die Fachpersonen in einem Spannungsverhältnis zwischen Prävention und Intervention, da sie jeweils beurteilen müssen, welche Handlungen oder »Vorfälle« »normal« sind. Zudem stehen sie unter dem Erwartungsdruck ihrer Auftraggeber und der Öffentlichkeit. »Falsche« Erwartungen, d.h. Erwartungen, welche die Fachpersonen im jeweiligen Rahmen ihrer Tätigkeit nicht erfüllen können, können ihre Arbeit durchaus torpedieren, da das Spannungsfeld zwischen Intervention und Prävention durch eine zusätzliche Dimension noch weiter aufgespannt wird. In dieser Hinsicht distanzieren sich die Fachpersonen von Präventionsverständnissen, die auf Wirksamkeit und Evidenz abzielen und die charakteristisch für kriminalpolitische Programmatik sind. Andererseits können ihre eigenen, am Kontext bzw. an Problemdeutungen entwickelten »Präventionsverständnisse« auch als Orientierung an kriminalpolitischer Programmatik angesehen werden, insofern als Prävention auf die Normalisierung von Jugendgewalt zielt und damit auf die Sicherung der »Professionen«. Eine Normalisierung von Jugendgewalt und die damit verbundenen Handlungsweisen im Kontext von Jugendgewaltprävention und auch Jugendgewalt »als alltägliche und gewöhnliche Erscheinungsformen zu lesen « (Kessl/Krasmann 2005: 240), sichert zwar das etablierte und sich etablierende »Präventionsinstrumentarium«, trägt aber trotzdem weiter zu einer »Dangerisierung« Jugendlicher bei (ebd.: 240). Das Bedürfnis, Jugendliche in Schutz zu nehmen, wird gewissermaßen genährt von der Kritik an gesellschaftlichen Skandalisierungsprozessen. Letztlich bleibt an dieser Stelle die Frage offen, ob Prävention eigentlich mehr sei als eine Form von Disziplinierung und warum es den Begriff der Prävention überhaupt braucht, worauf in der theoretischen Anschlussdiskussion noch zurückgekommen wird. Zunächst werden Deutungen von Jugendgewalt und Jugendgewaltprävention rekonstruiert, die auf der Ebene des »Nationalen Programms Jugend und Gewalt« entwickelt werden, und schließlich zu den Ergebnissen der bisherigen Interpretationen in Bezug gesetzt.

\subsection{Jugendgewaltprävention auf Ebene des Programms}

Bevor das Verhältnis zwischen sozialer Praxis und kriminalpolitischer Programmatik theoretisch diskutiert wird (4.3), werden hier zunächst die zentralen Aspekte herausgearbeitet, welche die Ebene des Programms betreffen. Die Aussagen beziehen sich insbesondere auf die erste der drei Konferenzen, da die Interviews im Anschluss an diese Konferenz geführt worden sind und 
dafür ein ausführliches Transkript vorliegt, sowie auf das Dokument »Gesamtschweizerisches Präventionsprogramm Jugend und Gewalt« (EDI/BSV 2010a). Die befragten Fachpersonen sind zum großen Teil aus der Liste der Ausstellerinnen und Aussteller sowie der Teilnehmerinnen und Teilnehmer der ersten Konferenz rekrutiert worden, teilweise liefen die Kontakte über daran anschließende Empfehlungen. Die zweiten und dritten Konferenzen werden anschließend hinsichtlich zentraler Unterscheidungsmerkmale beschrieben, wobei insbesondere die Inszenierung von Jugendgewalt durch »Jugendliche« thematisiert wird. Das »Nationale Präventionsprogramm Jugend und Gewalt« wurde seit Mitte 2015 nicht weiterfinanziert und ist beendet. Dafür wird ein anderes Programm, das »Nationale Programm Jugendmedienschutz Medienkompetenzen« (EDI/BSV 2010b), gefördert. Das Argument für diese Verschiebung liefert die Annahme, dass die Jugendgewaltraten zurückgegangen seien, bestimmte Formen von Jugendgewalt, v.a. sexuelle Gewalt im Kontext von Mediennutzung, jedoch zunähmen. Die damit einhergehende Annahme, dieser problematischen Form jugendlichen Handelns sei durch Kompetenzförderung entgegenzuwirken, plausibilisiert die Beobachtung der hier vorliegenden Arbeit, dass Jugendgewaltprävention nicht auf die Veränderung gesellschaftlicher Rahmenbedingungen, sondern des individuellen Handelns im Sinne einer Anpassung an gegebene Strukturen abzielt. In diesem Zusammenhang, so die These, werden gesellschaftliche Probleme über Risikofaktoren individualisiert und nicht nur in den Verantwortlichkeitsbereich der Fachpersonen, sondern auch der Jugendlichen gestellt. Hinweise für einen aktivierungspolitischen Hintergrund des Programms liefert auch die Information des Dokuments »Gesamtschweizerisches Präventionsprogramm Jugend und Gewalt«: »Das Programm und damit die Finanzmittel des Bundes sind auf fünf Jahre befristet. Nach Ablauf des Programms wird sich der Bund aus den verschiedenen Programmaktivitäten wieder zurückziehen.« (S. 13) So ist davon auszugehen, dass das auf fünf Jahre angelegte Programm anstrebt, die Zielgruppen, »die für Gewaltprävention verantwortlichen Kreise aus Politik, Verwaltung und Praxis auf Ebene Bund, Kantone und Gemeinden« (S. 9), im Sinne einer Übertragung von Verantwortung sowie Verantwortlichkeit zu aktivieren. ${ }^{2}$ Im Folgenden werden die Aussagen im Kontext des »Nationalen Präventionsprogramms Jugend und Gewalt« hinsichtlich ihrer Adressierungsweisen untersucht (4.2.1 bis 4.2.4) und in Bezug gesetzt zu den Ergebnissen der Interpretationen der Interviews (4.2.4 und 4.3). Dabei fokussieren die Interpretationen zunächst die Rahmenveranstaltung der ersten »Nationalen Konferenz Jugend und Gewalt« - da diese sich an ein breites Publikum, also Fachpersonen jeglicher Couleur, richtet, wohingegen spezielle Themen auf eher fachgruppenspezifischer Ebene im Rahmen von Workshops vertieft worden sind - sowie auf

2 | Zur Differenzierung von "Verantwortung" und "Verantwortlichkeit» vgl. Weyers 2006. 
das Dokument »Gesamtschweizerisches Präventionsprogramm Jugend und Gewalt«. Aus dieser Perspektive kommen v.a. die Fachpersonen und Jugendlichen als Adressatinnen und Adressaten des Sprechens über Jugendgewaltprävention auf kriminalpolitischer Ebene in den Blick, wobei die Fachpersonen als verantwortliche Subjekte angerufen werden. Ein Augenmerk wird anschließend auch darauf gelegt, inwiefern sich hierbei Veränderungen über die Zeit des Programms beobachten lassen, das auf eine Dauer von fünf Jahren angelegt ist - innerhalb dieser Zeitspanne wurden drei Konferenzen veranstaltet. ${ }^{3}$

\subsubsection{Zur Konstruktion von Kollektivität}

Die gesamtgesellschaftliche und fachbereichsspezifische Relevanz der Thematik wird über die Herstellung einer Kollektivität vermittelt. Dabei wird zudem deutlich, dass die Kriminologie und kriminalstatistische Untersuchungen den obersten Bezugspunkt des Programms darstellen. Kollektive Betroffenheit und daran anschließende kollektive Verantwortung werden bereits in den ersten Sätzen der Konferenz konstruiert, indem die Teilnehmenden mit einer Polizeistatistik zu Jugenddelinquenz konfrontiert werden. Diese Aussage vermittelt, dass alle Teilnehmenden diese Statistik kennen sollten und diese nicht in Zweifel zu ziehen ist.

"Sie habens ja alle gesehen Trendwende bei der Jugendgewalt - die Deliktquote bei den Gewalttätern geht um 30 \% zurück fragt man sich wie gesagt braucht es dieses Konferenz noch sind wir zu spät haben wirs verpasst hat sich das Thema sozusagen von selbst erledigt, ich denke sie alle, die sich mit dem Thema beschäftigen, wissen, nein so ist es nicht [...]."

In dieser Aussage wird die Erwartung eines kollektiven Wissens konstruiert und adressiert. Wiederholt wird das gesamte Publikum, »Sie alle« und »wir« als Wissende, angesprochen, das die neuesten Polizeistatistiken kennen und sich bereits darüber im Klaren sein müsste, dass sich das Thema angesichts der Statistik nicht von selbst erledigt habe. Die gemeinsame Wissensbasis wird dabei nicht in Zweifel gezogen, sondern normativ erwartet. Auch die Legitimation des Programms, dessen Gefährdung trotz des Blicks auf die Statistik für nichtig erklärt wird, wird nicht in Zweifel gezogen. Mit dem Verweis auf die Deliktquote wird der Problembereich des Programms auf diejenigen Fälle reduziert, die in der Polizeistatistik erfasst sind. Prävention wird folglich von vornherein auf kriminalitätsstatistische Untersuchungen gemünzt. Pro-

3 | Die Qualität der zu analysierenden Dokumente variiert aufgrund unterschiedlicher Dokumentationspraktiken und sprachlicher Barrieren. Die Konferenzen waren dreisprachig und wurden übersetzt. 
blematisiert werden damit also »Gewalthandlungen«, die polizeistatistisch erfasst sind. Allgemeine und soziale Präventionsverständnisse werden damit zu Beginn an den Rand gedrängt. Im Verlauf der Konferenz werden diese gemeinsame Wissensbasis und deren Gültigkeit zwar auch relativiert, zunächst jedoch werden sie aufgegriffen und reproduziert. Die (Be-)Deutung statistisch generierten Wissens als gesichertes Wissen wird insgesamt aufrechterhalten. Dem Programm gehe es nicht nur darum, »[...] zu zeigen, was wir tun und wie gut wir es tun, sondern wo müssen wir weiterarbeiten [...]«. Diese Aussage rekonstruiert die kollektive Betroffenheit. Die kollektive Verantwortung und Aufgabe bezieht sich dabei auf ein gemeinsames, auf die Zukunft gerichtetes Handeln, das "gut« sein soll. Dieser Zusammenhang betont nicht nur die Relevanz einer gemeinsamen übergeordneten Präventionsstrategie, sondern auch die Relevanz einer "guten « Prävention. Sie wird wiederholt aufgegriffen und manifestiert sich im Laufe der Konferenz: »Im Zentrum des Programms steht deshalb das voneinander Lernen Wissen Anderen zur Verfügung stellen, damit wir gemeinsam weiter gehen können und diese Prävention verbessern können und das Andere ist eben das Vermitteln von Wissen über erfolgsversprechende Präventionsmaßnahmen.« Auf der Basis gemeinsamen Wissens wird kollektive Verantwortung konstruiert, die gemeinsames auf die Zukunft gerichtetes Handeln fokussiert. Diese Anrufung geht über die Zuschreibung von bloßer Verantwortung hinaus, indem sie zudem über die Art und Weise der Umsetzung informiert: Prävention soll Erfolge zeigen, wobei ein ganz bestimmtes Wissen vonnöten sei. Wissen über Prävention wird gleichgesetzt mit Wissen über »erfolgversprechende Prävention«. Diese Aussage differenziert also zwischen »Prävention « und »erfolgversprechender Prävention« und ruft das Publikum somit nicht nur in seiner Verantwortung (was ist zu tun: Prävention) an, sondern auch in seiner Verantwortlichkeit (wie ist etwas zu tun: gemeinsam, gut und erfolgversprechend). Dieser Zusammenhang zwischen kollektiver Verantwortung und gemeinsamem auf die Zukunft gerichtetem, gutem und erfolgversprechendem Handeln wird zunehmend verfestigt:

"Ja wir haben es gehört, Frau V. hat es schon erwähnt wir können beobachten dass die Gewalt zumindestens die registrierte Gewalt zurückgeht. Wenn dem so ist freu mich ich glaub wir haben gar nichts verpasst ich denke auch die existierenden Projekte und Programme haben bereits hier einen Beitrag geleistet aber wenn es dann zu Gewalttaten kommt, kann das gravierende Folgen für Täter, Opfer und das Umfeld haben und ich glaube auch deshalb genau deshalb müssen wir dranbleiben und alles daran setzen Gewalt zu verhindern besten Dank."

Die Relativierung des Vertrauens auf die Polizeistatistik tut der Bedeutung der kollektiven Verantwortung der Teilnehmenden und des auf die Zukunft gerichteten Handelns sowie der Legitimation des Programms keinen Abbruch. 
Dass sich ein Rückgang »registrierter Gewalt« zeigt, bestätige vielmehr, dass das Programm und seine Beteiligten auf dem richtigen Weg seien. Folgend wird an die "geschätzten Verantwortungsträgerinnen und Verantwortungsträger« appelliert, die auf Basis des gemeinsamen Wissens darüber, dass es keine Patentlösungen gebe und Jugendgewalt ein vielschichtiges Problem sei, sich für ein »gemeinsames ein koordiniertes Vorgehen über alle Staatsebenen hinweg« einzusetzen. »[...] Bund, Kanton, Städte und Gemeinden Anfang 2011 das gemeinsame Präventionsprogramm Jugend und Gewalt gestartet [...].« Die Konstruktion von Kollektivität, insbesondere hinsichtlich kollektiver Betroffenheit und der Bedeutung gemeinsamen und guten Handelns in der Zukunft, steht im Zusammenhang mit der Legitimation des Programms. Trotz sinkender Jugendgewaltraten sei Jugendgewaltprävention notwendig. Die Statistik spreche sogar für den Erfolg von Jugendgewaltprävention und daher für die Bedeutung, Jugendgewaltprävention weiter zu stärken. Erfolg, der sich in der Statistik niederschlägt, wird hier als ein zentrales Kriterium für die Legitimation von Jugendgewaltprävention und die Notwendigkeit, im Rahmen eines Programms gemeinsam zu handeln, erachtet. Dies zeigt sich auch bei der dritten Tagung, auf der trotz zunehmenden Rückgangs von Jugendgewaltquoten und der Beendigung des Programms an das kollektive Interesse appelliert wird, auch in der Zukunft weiter zusammenzuarbeiten, da sich der Erfolg ja an der Statistik gezeigt habe. Damit wird ein Präventionsverständnis entwickelt, das sich auf Risiken bezieht, die sich in kriminalstatistischen Untersuchungen zeigen. Spätestens hier wird deutlich, dass das »Nationale Präventionsprogramm Jugend und Gewalt« mit der in Deutschland verbreiteten kommunalen Gewalt- bzw. Kriminalprävention zwar Ähnlichkeiten aufweist, aber auch zentrale Unterschiede. So richtet sich das »Nationale Präventionsprogramm Jugend und Gewalt« nicht an ein öffentliches, sondern an ein Fachpublikum. Es zielt auf eine Schwächung föderaler Strukturen. Zwar werden Kantone, Städte und Gemeinden in ihrer Verantwortung angerufen; durch die Vermittlung, wie Prävention durchzuführen ist, also durch die Fokussierung auf »gute« und »erfolgversprechende « Prävention, die sich auf Risiken polizeistatistisch erfasster Delikte bezieht, werden sie jedoch in der Vielfalt der ihnen zur Verfügung stehenden Maßnahmen eingeschränkt. So kann das »Nationale Programm Jugend und Gewalt« nicht als

"kriminalpolitische Bewegung beschrieben werden, deren Initiatoren und Hauptakteure dem polizeilichen oder innenpolitischen Behördenapparat auf kommunaler oder Länderebene zuzurechnen sind. Die Initiativen für ressortübergreifende Kriminalprävention kommen in vielen Fällen 'von unten`, ihre Durchführung hing und hängt an einzelnen Einrichtungen oder am Engagement einzelner Personen. Deshalb unterscheiden sich die Modelle und die Entstehungsprozesse je nach den Iokalen Traditionen, Strategien und Gegebenheiten von Kommune zu Kommune. Die Heterogenität der verschiedenen 
kriminalpräventiven Projekte spiegelt sich in den vielfältigen Bezeichnungen für die Präventionsmaßnahmen auf Länder- oder auf kommunaler Ebene." (Berner/Groenemeyer 2003: 85)

Zwar ist das Programm inhaltlich gesehen kriminalpolitisch (formal: sozialpolitisch) und orientiert sich in diesem Sinne an polizei- und kriminalstatistischen Untersuchungen; die »Bewegung « kommt hier jedoch vielmehr »von oben « und zielt auf die gleichzeitige Stärkung und Schwächung einzelner Fachbereiche ab. Die Heterogenität von Maßnahmen ist zwar, wie die Datenlage der hier vorliegenden Arbeit plausibilisiert, sehr vielfältig (vgl. ebd.: 85), jedoch wird genau diese Heterogenität »von oben« problematisiert. Möglicherweise bekommen die dazu vorliegenden Studien diese Differenzierung aufgrund der Beschränkung auf die Analyse von Programminhalten und dieser vorangegangenen theoretischen und methodologischen Überlegungen nicht in den Blick (vgl. Finkel 1995; Steinert 1995). Mit dem Konzept der Subjektivierung, das in der vorliegenden Arbeit als zentrales analytisches Konzept fungiert, kommt hingegen in den Blick, dass die Zuschreibung von Verantwortung und Verantwortlichkeit bemächtigt und entmächtigt, da diese Adressierung gleichzeitig Kontingenz reduziert. So wird über die Problematisierung von polizei- und kriminalstatistisch gemessenen Delikten sowie über den Appel an gemeinsame, gute und erfolgreiche Präventionsarbeit Prävention eine ganz bestimmte Prägung gegeben. Im Folgenden wird gezeigt, wie auf Programmebene neben der Reproduktion kollektiver Verantwortung und Verantwortlichkeit das »Problem der Jugendgewalt« weiter ausdifferenziert wird. Dabei wird auch die Gültigkeit statistisch generierten Wissens und vereinseitigender Erklärungsmuster problematisiert und kritisiert, was der Bedeutung des Programms jedoch keinen Abbruch zu tun, sondern diese sogar erst recht zu legitimieren scheint: »[...] Einstieg gefreut dass Sie berichten Frau V. Gewalt ist zurückgegangen ja das ist ja wunderbar wir sollten nicht daran denken dass wir jetzt aufhören wir wissen nicht genau ob das unsere Programme sind aber es schafft eine gewisse Gelassenheit im Umgang mit dem Thema [...].« Indem ein gemeinsamer Verantwortungsbereich, der sich auf die Zukunft richtet, zunehmend institutionalisiert wird und sich Wissensbereiche hinsichtlich des Gegenstands öffnen, können möglichst viele Akteure unterschiedlichster Bereiche angesprochen werden, nicht nur bezüglich Verantwortung und Verantwortlichkeit, sondern auch der Sicherung der eigenen »Profession«, wie noch aufgezeigt wird. Zunächst wird festgehalten, dass für die Konstruktion von Kollektivität ein Sinnbild gebraucht wird, auf das auch in den folgenden Tagungen zurückgegriffen wird. Die Metapher, dass alle im selben Boot sitzen, verdeutlich die Bedeutung gemeinsamen Handelns, schreibt diesem sogar eine existenzielle Bedeutung zu: 
"[...] und ich vergleiche das oftmals mit dem Prozess des großen Ozeandampfers wenn ich auf etwas zusteuere und ich entscheide mich ich reiße das Steuer nach rechts dann muss ich es nach rechts halten wenn ich kurz nachdem ich das Steuer rechts eingeschlagen habe gerade wieder nach links drehe und nachher wieder nach rechts drehe und wieder nach links drehe dann erreiche ich garantiert den Eisberg wenn ich aber nach rechts drehe und dann beginnt die träge Masse sich langsam zu kehren und genau das ist das Bild das ich mitnehme und das ist das Bild für den Alltag bei uns in unserer täglichen Arbeit bei welcher es streng ist den Kurs rechts weiter zu halten weil die Politik hier oft mal etwas anders fordert aber man muss hingehen uns sagen wir machen jetzt das uns so jetzt weiterfahren und die Innovationen auf diesem Kurs weitersetzen."

Dieses Szenario ist eine Auseinandersetzung mit der Bedrohung des Kollektivs durch den Eisberg - eine diskursiv etablierte Metapher für Gewalt - und appelliert nicht nur an gemeinsames, auf die Zukunft gerichtetes »richtiges« bzw. »richtig gerichtetes« Handeln, sondern auch an die Konsequenz, etwas einmal Begonnenes zu Ende zu führen. Das Bild, gemeinsam in einem Boot einem Eisberg auszuweichen, beschreibt nicht nur die Bedeutung gemeinsamen Handelns, sondern auch dessen Zwang. Jugendgewalt wird damit als eine existenzielle Bedrohung dargestellt. Der Eisberg lädt nicht ein, das eigene Handeln zu reflektieren oder zu diskutieren, sondern einen einmal eingeschlagenen Kurs nicht zu verlassen. Einzelne Maßnahmen sollten diesem Bild nach nicht an Probleme angepasst werden, sondern das Problem, das - hier nicht weiter differenziert - als ein einziger großer Eisberg dargestellt wird, soll gemeinsam umfahren werden. Dieses Bild rekonstruiert nicht nur die Bedrohung des Kollektivs, sondern verstärkt auch die Selbstreferentialität des Programms, das sich über die Skandalisierung von Jugendgewalt legitimiert, woran auch die befragten Fachpersonen anschließen.

"Gewalt kann dabei als ,Verdichtungssymbol zur Bezeichnung nahezu beliebiger Handlungsweisen und Sachverhalte verwendet werden, um diese zu diskreditieren und zu skandalisieren. Indem Probleme als , Gewalt bezeichnet werden, ist die moralische Empörung sicher, verstärken sich Solidaritätsgefühle der Ankläger und können neue Unterstützer mobilisiert werden. [...] Daran schließt regelmäßig die Metapher von der 'Spitze des Eisberges، an: ,Die Erweiterung des Falls erfolgt argumentativ, nach dem einfachen Muster des Assoziierens. Ein konkreter, spektakulärer, vielleicht auch extremer Fall wird als Teil eines größeren dahinter stehenden, bedeutsameren und bedrohlicheren Problems konzipiert. Verschiedenartige Phänomene werden in einer einleuchtend erscheinenden Weise als , Gewalt zusammengefasst und verweisen dadurch auf scheinbar ähnliche Erfahrungen, Probleme, Ursachen und Lösungen - allerdings um den Preis, von allen kontext- oder fallspezifischen Besonderheiten abzusehen." (Liell 2002: 8; zit.n. Cremer-Schäfer 1995: 27) 
Dem Kollektiv steht der »Einzelkämpfer« gegenüber, der angesichts der Menge neuer Programme Sorge bereitet, womit wiederum an kollektive Zusammenarbeit appelliert wird:

"[...] es gibt sehr viel wahnsinnig viel verdammt viele Programme der Prävention das ist zu Ihrer aller Ehre was da Welt herumwabert ich bin immer etwas besorgt wenn neue Programme aufgeschaltet werden und wenn man da als Einzelkämpfer in See sticht die Sage mit geblähtem Segel sollten Sie sich vielleicht darauf besinnen dass Sie sich gegenseitig kräftemäßig absprechen [...]."

Diese Aussage greift die Bedeutung der Unterscheidung von »Prävention« und »erfolgversprechender Prävention« auf. Relevant ist demnach die Unterscheidung zwischen der Zuschreibung von Verantwortung und der Art und Weise, wie mit dieser Verantwortung umzugehen ist, also der Verantwortlichkeit. Der Einzelgänger, der als einsamer Seefahrer umher segelt, fungiert als Sinnbild für die Autonomie einzelner Institutionen, Organisationen, Schulen, Gemeinden oder Kantone und wird nicht nur als Bedrohung des Kollektivs wahrgenommen, sondern auch als Bedrohung der eigenen Existenz, da er sich auf diese Art und Weise »abschafft«. Aber nicht nur der »Einzelgänger« bedrohe das Kollektiv und seine »Professionen«, sondern auch der »Eisberg« als Metapher für Gewalt bzw. für eine bestimmte soziale Gruppe, die der Jugend, insbesondere eines bestimmten, »pathologischen« Teils der Jugend, wie in Kapitel 4.2.2 weiter vertieft wird. Der Zusammenhang von Verantwortung und Wissen bündelt sich auch in dem Dokument »Gesamtschweizerisches Präventionsprogramm Jugend und Gewalt«, das die »verantwortlichen Akteure [...] zur Bekämpfung von Jugendgewalt« (EDI/BSV 2010 a: 3) aufruft. Der Zusammenhang zwischen Verantwortung hinsichtlich »Jugendgewaltprävention« und Wissen über Jugendgewaltprävention thematisiert also nicht nur die Verantwortung bestimmter Akteure, sondern auch die Art und Weise, wie sie mit dieser Verantwortung umzugehen haben, nämlich »kämpferisch«. Im Zusammenhang mit Wissen wird die Zuschreibung von Verantwortung zu einer Zuschreibung von Verantwortlichkeit. Damit ist die Vermittlung von Wissen als eines der primären Ziele des Programms anzusehen. So zielt das Programm auf eine Reduktion von Unsicherheiten bezüglich des Wissens ab, »welche Maßnahmen schweizweit bestehen, welche Wirkungen diese erzielen und wie diese zielgerichtet verbessert und optimiert werden können « (ebd.: 3). Auch hinsichtlich der Ursachen von Jugendgewalt besteht Unsicherheit (vgl. ebd.: 5), jedoch nicht über die Angemessenheit der Fragestellung nach »Ursachen und Ausmaß von jugendlichem Gewaltverhalten« (ebd.: 2) und der Annahme, dass das Wissen um »Gewaltrisiken« auf Basis statistisch generierter »Risiko- und Schutzfaktoren« (ebd.: 3) zentral sei. Auch Schreiber beobachtet im Kontext von kommunaler Kriminalprävention die Bedeutung der Hervor- 
bringung von Kollektivität. Sie spricht dabei von einem »Präventionskollektiv« (Schreiber 2011: 146). Dabei stellt auch sie Anrufungsprozesse in den Fokus ihrer Beobachtungen und macht auf die kollektive Orientierung an der Zukunft aufmerksam:

"Prävention schließt nicht aus, sondern ruft uns alle als Präventionssubjekte über die Einigung auf eine gemeinsame Zukunft hin an. Entscheidend für die Formierung von Gleichgesinnten ist daher nicht die Frage, wer wir heute sind, sondern wer wir zukünftig sein wollen. [...] Prävention bietet ein Zukunftsmodell an, das den Widersprüchen der modernen Welt trotzt, indem sie sie in die eigene Sache zu integrieren vermag. So würde eine einfache Sortierung von Menschen in Präventionssubjekte und -objekte den Präventionsdiskurs schwächen und seine Glaubwürdigkeit in Frage stellen. Selten legt sich der Diskurs auf konkrete Figuren fest. Denn prinzipiell sollte jede_r angesprochen werden, sich jede_rals Subjekt der Prävention erfahren lernen. In Bezug auf das Zukünftige haben wir alle die Möglichkeit, an Prävention mitzuwirken. Prävention schließt also ein. Sie integriert die Gefährdung in ihre Ordnung, provoziert nicht den Widerspruch, sondern macht inn sich produktiv." (Ebd.: 146f.)

Die hier vorliegende Arbeit macht jedoch die Beobachtung, dass die Fachpersonen auf der Ebene des Programms als Subjekte in ihrer Verantwortung und Verantwortlichkeit angerufen werden und dabei die soziale Gruppe der Jugendlichen objektiviert wird. Jugendgewaltprävention schließt aus, indem sie bestimmte Präventionsverständnisse bzw. spezifische Präventionskonzepte von vornherein suspendiert, damit bestimmten Organisationen und Institutionen die Legitimation entzieht und folglich auch deren Adressatinnen und Adressaten Hilfe und Partizipationsmöglichkeiten verwehrt. Das zeigt sich nicht nur auf der Ebene des Programms, was im folgenden Kapitel (4.2.2) weiter vertieft wird, sondern auch auf der Ebene der Interviews, in denen die Jugendlichen von der Mehrheitsgesellschaft abgegrenzt und damit von verschiedenen Möglichkeiten der Beteiligung potenziell ausgeschlossen werden. Diese Beobachtung wird auch in den theoretischen Diskussionen aufgegriffen. Das Programm betont zudem die Differenzierung zwischen »der Jugend « und »der besonders bedrohlichen« Jugend, wodurch jedoch der Kreis der Adressatinnen und Adressaten im Unklaren gelassen wird. Denn wie ist diese Unterscheidung in der Situation zu treffen? Das Programm macht damit potenziell eine große Gruppe für Maßnahmen zuschreibbar: Jugendliche sind somit eben nicht Subjekte kriminalpolitischer Programmatik, sondern Objekte, die beliebig adressiert - aber bezüglich Interventionen jederzeit (da die Gefahr omnipräsent ist) anrufbar gemacht werden - und somit subjektiviert werden können, worauf im nächsten Kapitel (4.2.2) näher eingegangen wird. Die befragten Fachpersonen reproduzieren diese Haltung im Sprechen über ihre Praxis nur bedingt, da sie in Bezug auf ihre fachbereichsspezifischen Er- 
fahrungen primär Handlungsweisen problematisieren, kontextualisieren und die Bedeutung des einzelnen Falls betonen. In gewisser Weise spricht dies für eine widerständige Haltung oder zumindest für eine ambivalente Positionierung. Sie rekurrieren darauf, dass auch sie dazu angehalten sind, ihre »Professionen « zu sichern, was sie auch anstreben. Das zeigt sich an den Beschreibungen, wie notwendig es sei, Erfolge auszuweisen. Im Kontext des Sprechens über Jugendgewaltprävention (auf der Ebene der Interviews) kommen die Objekte des Diskurses auch als Subjekte in den Blick, insofern als an deren Verantwortung für individuelle Lebensgestaltung bzw. Prävention im Sinne einer »guten« und »gesunden« Lebensführung appelliert und darauf verwiesen wird, dass diesbezügliche Erfolge nur am konkreten Fall und retrospektiv beurteilt werden können. In dieser Hinsicht wird auch Castels Beobachtung, dass »sich die präventiven Politiken nicht mehr in erster Linie mit Individuen, sondern mit Faktoren, mit statistischen Korrelationen heterogener Elemente befassen« (Castel 1983: 61; vgl. auch Schreiber 2011: 159), plausibel. Nicht Individuen im Sinne individuellen Handelns werden problematisiert, sondern die soziale Gruppe der Jugendlichen bzw. eine bestimmte Teilgruppe. Auf der Ebene des Programms kommen Jugendliche somit nicht als Subjekte, sondern als Objekte in den Blick.

\subsubsection{Zur Konstruktion von "Jugend"}

Zunächst ist festzuhalten, dass sich das Kollektiv konstruiert in Abgrenzung zur sozialen Gruppe der Jugend im Allgemeinen und im Besonderen: »Die Jugend « steht »Gruppen mit Mehrfachproblematik« (EDI/BSV 2010a: 5) gegenüber. Dabei wird auf utopische und dystopische Szenarien verwiesen, die den Präventionsdiskurs tragen: Eigentlich ist die Jugend als »gut« anzusehen, jedoch stellt eine kleine Gruppe von Jugendlichen eine enorme Bedrohung dar, die sich nicht nur an das Kollektiv und seine »Professionen « richtet, sondern an diese als Stellvertreter einer friedlichen Gesellschaft. Die Differenz einer »guten« und einer »schlechten« Jugend im Kontext einer friedlichen Gesellschaft ist hier zentral und wird fortwährend reproduziert:

"(...) leben wir in einer sehr zivilen und sehr friedlichen Gesellschaft und auch bei den Jugendlichen aber das heißt nicht dass wir jetzt alle nach Hause gehen können und da n Fanta trinken können oder was auch immer sondern wir müssen wie es so schön heißt am Ball bleiben (...) wir haben etwa 7-10 \% junge Menschen mit doch erheblichen Problemen der Dissozialität das ist jetzt nicht nur Gewalt das ist Delinquenz und viele andere Phänomene die ja sehr relevant und sehr schädlich sein können für die Betroffenen (...)." 
Die Wir-Perspektive verweist wiederum auf eine Reproduktion kollektiver Betroffenheit, die sich zunächst darauf bezieht, in einer »sehr zivilen und friedlichen Gesellschaft « zu leben. Dies trifft in gewisser Weise auch auf »die Jugendlichen $\ll$ zu, die hier jedoch nicht als Teil des Kollektivs adressiert werden. Ein kleiner Teil, »etwa 7-10 \% junger Menschen mit doch erheblichen Problemen der Dissozialität«, wird nochmals abgegrenzt und problematisiert, insofern als sie eine Bedrohung für »die Betroffenen« darstellen. Wer die Betroffenen im dem Fall sind, wird nicht expliziert. In diesem Kontext lässt sich die Bedrohung also auf das Kollektiv als Stellvertreter einer »zivilen und friedlichen Gesellschaft« beziehen, insbesondere deshalb, weil dieser eine Gruppe gegenüberstehe, die sich durch »Dissozialität« auszeichnet. Besonders auffällig ist die Formulierung eines Besitzanspruchs, der im Laufe der gesamten Konferenz präsent ist und sich sowohl in Bezug auf die Gruppe der »guten«als auch der »schlechten« Jugendlichen richtet.

"[...] wir haben eine ganz vorzügliche Jugend natürlich haben wir so ein paar schwarze Schafe multiple Rückfälligkeiten an den Tag legen die uns das Leben etwas sauer machen aber ohne sie wären wir ja auch arbeitslos also sollten wir innen auch ein bisschen Tribut zollen (.) eine sehr gute gesunde Jugend haben wir man sagt es viel zu wenig denke ich [...]."

Auch das Bild der »schwarzen Schafe«, das von einer »vorzüglichen Jugend« bzw. »sehr gesunden Jugend « unterschieden wird, kehrt über alle Konferenzen hinweg wieder. Hier tritt die Differenzierung zwischen »normaler« und »pathologischer« Jugend bzw. Jugendgewalt besonders deutlich hervor, die auch im Dokument »Gesamtschweizerisches Präventionsprogramm Jugend und Gewalt« als zentrales Unterscheidungskriterium dient: »Aggressive Verhaltensweisen gehören zum normalen Verhaltensrepertoire von Kindern und Jugendlichen, sofern sie sich nicht verfestigen und über ein gewisses Mass hinausgehen. Problematisch sind jene Fälle, in denen sich gewalttätiges Verhalten manifestiert und einen bestimmten Schweregrad erreicht.« (EDI/BSV 2010a: 4) Das »Pathologische«, Schwarze«, wird hier eigentlich nicht abgegrenzt vom »Normalen«, sondern von etwas, das besser ist als »normal«, nämlich »sehr gesund « und »vorzüglich«. Der Anspruch, eine »gute Jugend $\mathrm{zu}$ haben«, scheint nicht hoch genug zu sein: Jugend soll »vorzüglich« und »sehr gesund « sein. Hier tritt auch das ambivalente Verhältnis zu der »pathologischen« Gruppe deutlich hervor, da sie zwar das kollektive Leben vermiese, aber auch existenziell sichere. Dies ist eine zentrale Beobachtung, die sich über die gesamte Konferenz hinweg machen lässt. Nicht primär Gewalt im Sinne (jugendlichen) Gewalthandelns wird problematisiert, sondern eine bestimmte soziale Gruppe der Jugendlichen selbst. Diese bedrohe zwar das Kollektiv, »ernähre« jedoch auch gleichzeitig seine »Professionen«. Dieser »dissoziale« oder 
allgemeine »pathologische« Teil der Jugendlichen wird damit gleichzeitig als Bedrohung und Sicherung wahrgenommen. Sicherheit wird damit über die Konstruktion eines Bedrohungsszenarios inszeniert:

"[...] für mich ganz beeindruckend in der Schweiz wie viel es gibt wie engagiert man zu Werke geht äh es war die Frage mit Recht sonst würden wir ja arbeitslos Gewalt ernährt in gewisser Weise auch Ihre Professionen es kam heute Nachmittag bei dem Schulforum eine sehr interessante Frage auf ja wenn dieses PFADE Projekt wir arbeiten auch mit außerschulischen Versionen davon wenn das s0 erfolgreich ist werden dann nicht die Schulsozialarbeiter arbeitslos ich kann sie beruhigen die Probleme werden weitergehen sie werden manchmal stärker, manchmal bissl schwächer sein und wir müssen auch aus meiner Sicht und das nehm ich auch mit das kam mehrfach das Positive nicht immer nur die negative Seite der Entwicklung unserer Jugend sehen es ist eine kleine Kerngruppe die wir auf den richtigen Pfad bringen müssen und die ernährt in gewisser Weise selbst die Hochschulen nämlich meine Universität 'Name einer Universitätı einen Lehrstuhl innehatte wäre nicht nach ,Name einer Stadt gekommen wenn die Jugend nicht ein bissl gewalttätig gewesen wäre in Name einer anderen Stadtı."

Nicht nur die eigene »Profession«, sondern auch die im Publikum vertretenen »Professionen« würden von den gewalttätigen Jugendlichen »ernährt«, was wiederum als Appell an kollektive Betroffenheit, im Sinne einer Legitimation, sich fortwährend mit dem Thema auseinanderzusetzen, angesehen werden kann. Hier wird mit der Sorge aufgeräumt, dass erfolgreiche Präventionskonzepte die Probleme lösen könnten: »Die Probleme werden weitergehen«. Jugendgewalt wird dabei in hohem Maße normalisiert. Es wird als kollektive Pflicht angesehen, die jeweiligen »kleinen Kerngruppen auf den richtigen Pfad « zu bringen. An dieser Stelle wird daran erinnert, dass die im Rahmen der hier vorliegenden Arbeit befragten Fachpersonen sich hinsichtlich ihrer Problematisierungsweisen von Jugendgewalt und ihren eigenen Präventionsverständnissen und -konzepten von kriminalpolitischer Programmatik zwar in kritischer Weise abgrenzen, indem sie eigene Deutungen und Relevanzen von äußeren Erwartungen differenzieren. Bezüglich der Logik von Jugendgewaltprävention, die in der Bestandssicherung der (eigenen) »Professionen« sowie der generationalen Ordnung und der gesellschaftlichen Strukturen liegt, gehen sie jedoch mit kriminalpolitischer Programmatik konform. Auf beiden Ebenen zielt Prävention letztlich auf die Anpassung Jugendlicher an gegebene Strukturen ab. Wie und ob das zu bewerkstelligen und zu messen ist, ist eine andere Frage. Dabei rekonstruieren die befragten Fachpersonen zwar auch eine Differenz zwischen »normalen« und »pathologischen« jugendlichen Gewaltformen, jedoch ist diese Differenzierung nicht entscheidend für ihre Präventionsarbeit. Das Kollektiv situiert sich also in ein beständiges Bedrohungsszenario, das seine Existenz sichert. Wie ist die Bedrohung genau beschaffen? 
Wie werden Jugendliche dabei adressiert? Die Äußerungen greifen auf Bedrohungsszenarien zurück, welche die kollektive Betroffenheit vertiefen und Verantwortung ins kollektive Bewusstsein stellen. Ein Bild, das sehr deutlich ins Zentrum rückt, ist die hohe Präsenz Jugendlicher im öffentlichen Raum zu bestimmten Zeiten und in Zusammenhang mit Alkohol: »Jedes Wochenende strömen beispielsweise 10oode von Jugendlichen in die größeren Städte in den Ausgang.« Anderen Beobachtungen nach

"[...] kommen Freitag Samstag Sonntag an einem schönen Sonntag oder an einem schönen Wochenende [...] in der Stadt , Name einer Stadt، bis zu 25 Jahre bis zu $100000 \mathrm{Ju}$ gendliche die in den Ausgang gehen und die sich vergnügen in dieser Stadt 100000 das ist eine Rießenzahl da wäre jede andere Gemeinde oder viele andere Gemeinde wenn sie dieses auf einen Schlag haben massiv überfordert wenn die Polizei nur 1 Promill von diesen 100000 Jugendlichen sich damit beschäftigen muss intensiv beschäftigen muss dann ist die Polizei schon sehr stark an ihrem Limit [...]."

Im Gegensatz zu den vorigen Beschreibungen geht die Bedrohung hier weniger von der pathologisierten Gruppe der Jugendlichen aus, sondern vielmehr von der »Masse« an Jugendlichen, die zu bestimmten Zeiten in den öffentlichen Raum strömen. Als bedrohlich werden dabei nicht bestimmte Handlungsformen problematisiert, sondern allein die starke Präsenz Jugendlicher im öffentlichen, städtischen Raum. Diese Beschreibung transportiert eine Sorge um die Polizei, die überfordert sein könnte, wenn sie sich nur mit »1Promill« dieser Masse an Jugendlichen beschäftigen müsste. Weiter problematisiert wird der Alkoholkonsum der Jugendlichen.

"Ein Großteil der Gewalttaten unter Jugendlichen ereignet sich zu diesen Zeiten an einigen wenigen Brennpunkten. Das ist in allen größeren Städten gleich. Die Städte haben aber wenige Kompetenzen und Handlungsspielraum um diese Probleme dann auch wirklich angehen zu können. Beispiel Alkohol. Bei den meisten Gewalttaten die Jugendliche begehen ist heute Alkohol im Spiel."

Auch hier wird eine Besorgnis kundgetan, und zwar um die Städte, die überfordert sein könnten, wobei unklar bleibt, womit eigentlich genau. Folgende Sequenz ist eine der wenigen, die »das Problem« genauer beschreibt, jedoch auch dramatisiert.

"[...] nun die Pluspunkte ich habe deren drei wie die heilige Dreifaltigkeit erster starker Punkt erster Pluspunkt der mich herausgefordert ist die Tatsache dass diese Gewalt in aller Leute Mund sei sie sei nicht im Steigen begriffen ich kann das auch statistisch belegen sie nimmt eher ab aber es gibt sie trotzdem diese Gewalt also muss man darüber reden die Gewalt verändert mitunter ihr Antlitz insofern als man sieht dass die Jugend- 
lichen vermehrt zuschlagen sie wollen die Leute niedertreten sie haben zum Teil auch blanke Waffen und Alkohol ist immer mit im Spiel in solchen Schlägereien in solchen Raufereien die vor den Kadi gelangen es gibt immer Alkohol oder andere Suchtmittel Betäubungsmittel die mit im Spiele sind [...]."

Gewaltverhalten nehme zwar nicht zu, wie eben die Statistik zeigt, verändere aber ihr »Antlitz«. Die Jugendlichen würden vermehrt körperlich gewalttätig - dies mit Absicht - und würden sich »blanker« Waffen, Alkohol und anderer Suchtmittel bedienen. Diese Bedrohungsszenarien seien Realität und Normalität. Vorstellbar sind jedoch auch Szenarien, die in ihrem dystopischen Charakter noch stärker ausgeprägt sind.

"[...] auch ,Name einer Stadt ‘ wo ich jetzt in den letzten 7 Jahren gelandet bin wäre nicht ,Name der selben der Stadt die Universität wenn nicht in 'Name einer anderen Stadt die Ausschreitungen des jungen Menschen gewesen wären so dass eine kleine Gruppe von Studenten und Dozenten in das verschlafene Städtchen gezogen ist wenige Jahre begannen dort auch die Ausschreitungen also es gehört in gewisser Weise zur Jugend dazu und wir müssen differenzieren diese schweren und gravierenden Entwicklungen von diesen alltäglichen Aggressionen die auch im Tierreich dazugehören (.) Kämpfe, sich balgen, Rangordnungen definieren und da ist ein bisschen die Gefahr dass wir vielleicht manchmal überreagieren und normale Entwicklungsphänomene pathologisieren was ich als Ausblick noch sagen möchte wir müssen auch die strukturellen Aspekte so sehr wir Familie Schule weil ich eben genuin Psychologe bin aber sehen sie an wenn wir in Spanien 45 \% Arbeitslosigkeit junger Gebildeter haben dann ist nicht auszuschließen dass irgendwann hier auch Dinge explodieren die nicht mehr durch die herkömmlichen Erklärungsmuster falsch erzogen und so weiter sondern durch strukturelle Probleme immer und das ist vielleicht eines der nächsten Themen das bei uns auf der Tagesordnung stehen wird."

Einer Normalisierung und Naturalisierung von Jugendgewalt (im Sinne einer biologischen Grundstruktur), die »auch im Tierreich dazugehören«, stünden »pathologische« Formen gegenüber, die hier als »Ausschreitungen« bezeichnet werden und deren Ursache »strukturelle Probleme« seien, die nicht als herkömmliche Erklärungsmuster fungieren. Von einer »natürlichen« Jugendgewalt seien organisierte Handlungsformen abzugrenzen. Die Bedrohung durch diese Form von »Jugendgewalt« bezieht sich darauf, dass es sich dabei eben nicht um »natürliche« Gewaltformen handele, die biologisch erklärbar seien. Die Bedrohung bezieht sich also auf Ungewisses, Unvorhersehbares, das seine Ursachen in »strukturellen Problemen« habe. Hervorzuheben ist, dass hier jedoch nicht die strukturellen Bedingungen der Gesellschaft hinterfragt und kritisiert werden, sondern das Handeln bestimmter Jugendlicher, die als soziale Gruppe selbst zur Zielscheibe kriminalpolitischer Interventionen ge- 
macht werden. Jugendliche werden nicht als gefährdete, sondern das Kollektiv gefährdende soziale Gruppe konstruiert. Sie werden nicht als Teil der Mehrheitsgesellschaft angesehen, die sich von dem Programm mehr Sicherheit erhofft, im Sinne einer Aufrechterhaltung des Status quo, d.h. der gegebenen Machtverhältnisse, die auch die generationale Ordnung sichern soll. Die Beobachtung, dass der Status quo der Bedrohung des Kollektivs aufrechterhalten werden soll, macht auch Schreiber in ihrer Analyse kommunaler Kriminalprävention. »Wenn die Prävention bestehen will, muss sie notwendig unverwirklicht bleiben.« (Schreiber 2011: 145) Die Vision einer friedlichen Gesellschaft, die an ihren »Professionen«, die für Sicherheit sorgen, hängt und das Bedürfnis hat, Machtverhältnisse zu sichern, benötigt ein gewisses Maß an Bedrohung. In diesem Zusammenhang beobachtet Schreiber die Bedeutung, Gewalt in seiner Omnipräsenz zu visualisieren. Auch über die Naturalisierung und Historisierung von Jugendgewalt wird diese zu einer omnipräsenten Kategorie, welche reproduziert wird, was die Fachpersonen, die im Rahmen der vorliegenden Arbeit befragt werden, insofern tun, als sie Handlungsweisen problematisieren und kontextualisieren. Auch Schreiber kommt zu dem Schluss, dass Prävention

"als Steuerungsinstrument gar nicht bestrebt ist, Kriminalität gänzlich abzuschaffen. Vielmehr versucht sie, die Kriminalität und ihre Wahrnehmung in einem für das Funktionieren der Gesamtbevölkerung erträglichen Maß zu halten. Bevölkerung durch Prävention verwalten meint dann, die zuvor durch Konvention bestimmten Normgrößen einzuhalten." (Ebd.: 152)

Die Anrufung der Fachpersonen in ihrer kollektiven Verantwortung kann auch als Aktivierung durch einen moralischen Diskurs über Schuld interpretiert werden. Der »Einzelkämpfer« gefährde nicht nur das Kollektiv, sondern »schaffe sich selbst ab《: »Über das Moment der Aktivierung stellt die Prävention nicht nur die Forderung, Verantwortung zu übernehmen, sondern kommt auch zu einer moralischen Bewertung. Wer nichts tut, macht sich schuldig.« (Ebd.: 158) Die hier vorliegende Arbeit zeigt, dass die Fachpersonen im Kontext von Jugendgewaltprävention nicht nur hinsichtlich ihrer Verantwortung, sondern auch ihrer Verantwortlichkeit als Subjekte angerufen werden und dies im Sprechen über ihre Fachbereiche reproduzieren, indem sie Jugendliche hinsichtlich der Art und Weise, wie ein »gutes Leben« zu führen sei, adressieren. Dieser Beobachtung nach machen sich die Subjekte im Kontext von Jugendgewaltprävention nicht nur schuldig, wenn sie nichts tun, sondern auch, wenn sie etwas tun, aber »falsch«. 


\subsubsection{Legitimationsweisen}

Dystopien, so macht Schreiber die Beobachtung, dienen der Legitimation von Prävention:

"Die Anrufung dystopischer Elemente bildet die notwendige Voraussetzung für die Rechtfertigung präventiver Interventionen. Prävention lebt davon, in den Menschen Ängste hervorzurufen. Erst die Angst vor einer unsicheren Zukunft legitimiert Ordnungsphantasien, deren Resultat die freiwillige Unterwerfung des Selbst unter die Handlungsmaxime der Prävention ist." (Schreiber 2011: 146)

Neben der Metapher des Eisbergs, dem gemeinsam entgegenzusteuern ist, kommen hier weitere dystopische Szenarien in den Blick, die auf die Zukunft verweisen. Die Utopie wird hingegen in der Gegenwart verortet - in »unserer« Gesellschaft, die »friedlich« sei. Andere Länder werden als Beispiele für Kollektive in den Blick genommen, die mehr als nur bedroht seien. Diese Bedrohung rührt u.a. von der Unmöglichkeit her, Jugendgewalt auf herkömmliche Weise, eben als biologische, kulturelle oder historische Konstruktion, zu erklären und zu normalisieren. Die Angst davor, die eigene Gesellschaft und deren Strukturen anzugreifen, infrage zu stellen und dort mögliche Ursachen für Jugendgewalt auszumachen, tritt hier deutlich hervor. Die Unsicherheit - die Gewalt - wird außerhalb der eigenen Gesellschaft verortet. Ähnlich wie Schreibers Beobachtung, dass sich Prävention in Bezug auf Zukunft legitimiert und es ihr »gelingt, sich durch die Berufung auf die Zukunft, sich einer Bewertung im Jetzt zu entziehen« (ebd.: 147), stellt die vorliegende Arbeit in Bezug auf die Frage der Bewertung und Legitimation auch rückblickend, angesichts der Kriminalstatistik, die eigentlich auf eine Minderung der Bedrohungslage hinweist. Die hieraus möglicherweise resultierende Legitimationskrise wird jedoch überdeckt durch die Versicherung darüber, dass der Status quo, d.h. auch die »Professionen«, bereits »gut« ist und es deshalb notwendig ist, nichts zu ändern. Hier wird das Bestreben verstärkt, die eigene Gesellschaft und ihre Prävention nicht zu verändern und zu hinterfragen: »Positive Entwicklungen der Kriminalstatistik werden als Effekt gelungener Prävention ausgedeutet. Die Bezugnahme auf Instrumente der quantitativen Wissenschaft leistet, dass sich die Prävention als seriöse Wissenschaft präsentieren kann.« (Ebd.: 153) So wird die zu Beginn der Konferenz gemachte Beobachtung, dass die Delikte bezüglich Jugendgewalt zurückgegangen seien, nicht als Bedrohung, sondern als eine Bestätigung des Programms dargestellt. Eine weitere zentrale Legitimationsgrundlage der hier vorliegenden Äußerungen wird aus der ökonomischen Kalkulation gezogen, die besagt, die Kosten der Prävention seien geringer als die der Repression. Angesichts der Tatsache, dass sich bereits historisch gesehen, Präventionsarbeit mehr und mehr professionalisiert und sich als sozialer 
Apparat etabliert hat, mag dies plausibel erscheinen. Ein Staat, der Steuern spart, ist Zeichen einer »friedlichen« und »guten« Gesellschaft. So misst sich den Beobachtungen der hier vorliegenden Arbeit nach gute Prävention nicht nur daran, dass sie »sich finanziell lohnt, uns in Arbeit bringt, Kosten senkt, neue Arbeitsplätze schafft« (ebd.: 169), sondern auch daran, dass sie den Status quo aufrechterhält. Somit enthält Prävention hier ein »doppelt normatives Versprechen«, nämlich »Entwicklungen abzuwehren, die normativ - nämlich als problematisch, gefährlich, ungerecht oder einfach schlecht - beschrieben werden und es wird versprochen, die Kontinuität und (relative) Unversehrtheit einer Gegenwart zu erhalten« (Reder/Ziegler 2011: 365$)$. Wobei sich die Unversehrtheit der hier gemachten Beobachtungen nicht auf die Unversehrtheit als Folge der Bändigung der Gruppe gewalttätiger Jugendlicher bezieht, sondern auf die Unversehrtheit des Präventionsapparats einer friedlichen Gesellschaft, der nur über die Konstruktion der Gruppe gewalttätiger Jugendlicher aufrechterhalten wird. In diesem Sinne lautet die Formel für Jugendgewaltprävention: Sie muss alles so verändern, dass alles beim Alten bleibt. Die Fachpersonen hingegen positionieren sich gesellschaftskritisch, woran sie hinsichtlich ihrer Konzepte von Prävention und der damit verbundenen Ziele und Ergebnisse jedoch nicht anschließen. Insofern positionieren sie sich in eine hoch ambivalente Situation: Einerseits haben sie Interesse an der Sicherung ihrer eigenen »Profession« und ziehen von der Orientierung an kriminalpolitischer Programmatik implizit und explizit Nutzen; andererseits sehen sie auch, dass Gesellschaft strukturelle Ungleichheit und somit Chancenungleichheit hervorbringt und dies jugendliches (Gewalt-)Handeln beeinflusst. Zwar kritisieren die Fachpersonen, dass Gesellschaft die Problematisierung von Jugendgewalt zu einem Dauerthema macht; sie sehen darin allerdings auch die Sicherung ihrer »Professionen«, die daran orientiert sind, Jugendliche in Schutz zu nehmen und ihnen »kontrollierte Lernfelder anzubieten. Ziele und Ergebnisse von Prävention betreffen jedoch letztlich das individuelle Handeln Jugendlicher, verstanden als Anpassungshandeln. Auf diese Weise wird Jugendgewalt als Deutungsmuster reproduziert und normalisiert, damit gesellschaftliche Ordnung und ihre Professionen aufrechterhalten bleiben. Demnach greift, entgegen Schreibers Beobachtungen (vgl. Schreiber 2011: 147), das utopische Szenario einer gewaltfreien Gesellschaft in der Zukunft hier nicht ganz: Auch eine Gesellschaft ohne Gewalt bedroht Gesellschaft, und zwar in substanzieller Weise. In der zweiten und dritten »Nationalen Konferenz Jugend und Gewalt« wird die Bedrohung rekonstruiert, indem die Bedeutung von Jugendgewalt als sinnlich erfahrbares und präsentes Phänomen im Kontext von Jugendgewaltprävention herausgestellt wird. Über Strategien der Visualisierung wird die Bedeutung von Jugendgewaltprävention an der Konstruktion von Jugendgewalt als omnipräsente Kategorie erfassbar. Damit wird sichtbar, was sonst unsichtbar ist (vgl. Münkler 2009: 8). Zwar spielen »Jugendliche« auf diesen beiden 
Konferenzen (im Gegensatz zur ersten Konferenz) eine Rolle, jedoch nur die ihnen zugeschriebene, inszenierte Rolle, die darin besteht, Jugendgewalt »vorzuführen« und ihren »Wirklichkeitscharakter« zu visualisieren, wie ein Ausschnitt aus dem Protokoll der zweiten Konferenz verdeutlicht: ${ }^{4}$

"Spricht über Programm der Stadt Lausanne ,ich und die anderen، (stark, knallig, zusammen) und einen Film, den Jugendliche im Rahmen dieses Projekts gemacht haben und in dem die Sicht auf und über Gewalt gezeigt wird. Die Jugendlichen wurden angeleitet, Name der Sprecherin ‘ betont jedoch, dass die Jugendlichen selbstständig agiert haben. 'Es gibt wirklich Jugendliche, die wirklich gut unterwegs sind،. Ein Ausschnitt des Films wird gezeigt: Junge Männer und Frauen stehen in mehr oder weniger zwei Gruppen im Dunkeln auf einem öffentlichen Platz, der wie ein Bahnhof aussieht. Zwei der jungen Männer aus verschiedenen Gruppen beginnen, sich anzuschreien. Nach einer Weile kommt die Polizei und hält die beiden jungen Männer, die sich aufeinander zubewegen, fest. Die jungen Männer versuchen mehrmals, sich von den Griffen der Polizisten zu lösen, die jedoch wieder und wieder die jungen Männer auseinanderhalten. Am Ende Iösen sich die Gruppen auf. Schließlich zeigt der Film, wie die Statisten miteinander diskutieren. Nach dem Film betreten eine junge Frau und ein Polizist ('Bürgerpolizist، in Zivil), die im Film als Statisten mitgewirkt haben, die Bühne und werden vom Moderator befragt. Die junge Frau erzählt von ihrer Vergangenheit: Sie sei schon viel mit der Polizei in Kontakt gekommen und der Meinung, die Jugendlichen wüssten nicht, dass sie beobachtet würden. Der Moderator fragt, wie es für sie und die anderen Jugendlichen gewesen sei, im Film mit der Polizei zusammenzuarbeiten. Die junge Frau erzählt, dass es anfangs komisch gewesen sei und dass es Jugendliche, v.a. Männer, gab, die nicht mit der Polizei zusammenarbeiten wollten. Der Moderator nimmt ihr das Mikrophon weg, obwohl sie noch nicht zu Ende gesprochen hat und gibt es dem Polizisten, der betont, es sei inm wichtig gewesen, in dem Film ein anderes Bild von der Polizei zu zeigen. Im Film gehe es um die Stärkung einer gegenseitigen Beziehung und um Verständnis. 'Was ist Wirklichkeit, was ist Abklatsch?، 'Zu wenig Werte, zu wenig Sensibilität.، Manchmal wolle man präventiv sein, aber dann käme die Gewalt und man könne nur noch repressiv sein."

Was vorgeblich als Engagement Jugendlicher angekündigt ist, wird nachträglich von der Polizei als Werbespot für die Polizei umgedeutet. Aus der Perspektive des Polizisten, der das Schlusswort in dieser Aufführung für sich beansprucht, dient der Film dazu, ein positives Bild der Polizei zu vermitteln - zu zeigen, wie die Polizei wirklich ist: Sie habe Interesse daran, die gegenseitige Beziehung und das Verständnis füreinander zu stärken und die »Wirklichkeit« zu zeigen. Der jungen Frau, der das Mikrofon noch während ihrer Schilderun-

4 | Dass Jugendliche im Rahmen der Konferenz nicht zu Wort kämen, wurde während der ersten Konferenz seitens des Publikums kritisiert. 
gen aus der Hand genommen wird, wird die Möglichkeit genommen, ihre Erzählung darüber zu Ende zu führen, dass dieses Projekt durchaus konflikthaft verlaufen sei und es Jugendliche und junge Männer gegeben habe, die nicht mit der Polizei zusammenarbeiten wollten. Die Möglichkeit der Artikulation wird ihr in dieser Inszenierung zwar zugestanden, jedoch nur bezüglich bestimmter inhaltlicher Kriterien, also quasi zensiert. Auch die dritte Konferenz gibt sich als Ort, an dem sich »Jugendliche« »ausdrücken« können. »Der Moderator weist auf die Bedeutung hin, Jugendliche im Rahmen dieser Tagung ins Scheinwerferlicht zu stellen und ihnen somit die Möglichkeit zu geben, sich auszudrücken. Hierbei handelt es sich um die Möglichkeit, sich künstlerisch auszudrücken.« Damit verweist der Moderator auf die Rahmung der Konferenz durch Stücke, die Studentinnen und Studenten der SSPSS (Fachschule für Sozial- und Pflegeberufe) aufführen. In diesen Stücken wird psychische und körperliche Gewalt in ihrem gruppenspezifischen Entstehungskontext inszeniert:

"Bevor die Moderation beginnt, führen Studentinnen und Studenten der SSPSS (Fachschule für Sozial- und Pflegeberufe) ein Stück auf. Sie tragen einheitliche dezente Kleidung und weiße Masken. In dem Stück geht es darum, wie eine Person, die 'gegen den Strich ' tanzt, von den anderen Personen ausgeschlossen (ausgelacht, geschubst, ausgegrenzt) wird. Die ausgeschlossene Person wirkt gedemütigt und traurig und sitzt am Ende zusammengesunken und allein da. [...] Studentinnen und Studenten der SSPSS führen ein Stück auf, wieder in dunkler einheitlicher Kleidung und weißen Masken. Es geht wieder darum, dass eine Person ausgegrenzt wird. Diesmal wird sie aber zusammengeschlagen, bis sie auf dem Boden liegt. Eine andere Person tritt weiter auf sie ein, bis sie sich nicht mehr bewegt. Andere Personen stehen drumherum, zeigen mit dem Finger auf die Person, die zusammengeschlagen wird und filmen die Szene mit ihren Handys."

Außer dieser Inszenierung von »Gewalt« wird die Konferenz von Studentinnen und Studenten der CSIA, einer Kunstgewerbeschule, begleitet, die sich so geschminkt haben, als seien sie kürzlich zusammengeschlagen worden. Fotos von dieser Maskerade hingen, auf große Plakate gedruckt, auf dem Konferenzgelände. Eines dieser Fotos war noch größer als Puzzle aufgestellt. Eine Seite des Puzzles zeigte die ungeschminkte, unversehrte, die andere Seite die geschminkte, versehrte Person. Die Besucherinnen und Besucher der Konferenz wurden dazu angehalten, die Puzzleteile zusammenzusetzen. Diese Art der Inszenierung ermöglicht die erneute Legitimation des Programms, das sich, insbesondere auf der dritten Konferenz, als die Beendigung des Programms bereits beschlossen worden war, verstärkt zu begründen versuchen muss und das seinen Fokus auf die Zukunft von Jugendgewaltprävention richtet, worauf bereits der Titel der Konferenz verweist: »Wie sieht die Zukunft der Gewalt- 
prävention in der Schweiz aus? « Eine Visualisierung sprachlich vermittelter dystopischer Szenarien verstärkt ein auf die Zukunft gerichtetes Sicherheitsbedürfnis und, in der Konsequenz, kollektives Handlungsbedürfnis. In der zweiten Konferenz zeichnet sich eine kritische Haltung gegenüber evidenzbasierten Projekten ab, wovon insbesondere zwei wissenschaftliche Rahmenvorträge zeugen. »Best« wird zu »Good Practice«, wobei »gute Prävention Maßnahmen sind, die mit wenig Aufwand Gewalt wirksam reduzieren«. Ein anderer Vortrag schließt mit der Feststellung, dass evidenzbasierte Forschung zu positiv beurteilt werde. Zweifel an Jugendgewaltprävention auf der Basis wissenschaftlicher Erkenntnisse könnten der Inszenierung von Jugendgewalt, der Wirklichkeitscharakter zugesprochen wird, von einer anderen, nicht wissenschaftlichen Seite entgegenkommen, um das Programm (zumindest retrospektiv) zu legitimieren. Die in der ersten Konferenz so starken utopischen und dystopischen Elemente weichen einer verstärkten Inszenierung von Jugendgewalt in der zweiten und dritten Konferenz: Jugendgewalt wird visualisiert und damit sinnlich erfahrbar gemacht, womit die Dringlichkeit von Jugendgewaltprävention (für die Zukunft) gestärkt werden soll. Mit Krasmann könnte die Tagung demnach als eine Heterotopie begriffen werden, als Ort, der das eigentlich nicht Sicht- und Sagbare zeigt und soziale Probleme damit über Sichtbarkeiten konstruiert:

"Das öffentliche Problematisieren bestimmter Phänomene als Gewalt kann Schlaglichter auf bestimmte Orte der Gewalt werfen und uns diskursiv vermittelt Einblick geben in diese ansonsten uneinsehbaren Orte (z.B. die private Wohnung). [...] Inwieweit strukturieren Diskurse die Wahrnehmung von Gewalt als Problem? Und wie wird umgekehrt das Erkennen von Gewalt als soziales Problem durch entsprechendes Sprechen darüber ausgeschlossen oder verhindert?“ (Krasmann 1997: 97)

Auch mit Herfried Münkler können Tagungen als »Orte bzw. Medien der Visibilität, die als Generatoren gesellschaftlicher Selbstvergewisserung dienen« (Münkler 2009: 7), verstanden werden. Dort wird ausgehandelt bzw. reproduziert, welche Formen von Jugendgewalt als problematisch anzusehen sind und als solche zum Gegenstand präventiver Maßnahmen gemacht werden sollen, etwa das Gesicht, dessen Entstellung wieder rückgängig gemacht werden soll, indem die Besucherinnen und Besucher der Konferenz die Puzzleteile »richtig« zusammensetzen. Die Zuschreibung von Verantwortung wird virulent, da sie sich auf ein Fachpublikum bezieht. »Die Praktiken und Techniken der Visualisierung können auf eine allgemeine Öffentlichkeit bezogen sein; genauso gut können sie aber auch auf eine Teilöffentlichkeit beschränkt bleiben, die sich auf diese Weise konstituieren und vom Rest der Gesellschaft absetzen.« (Ebd.: 8) In diesem Sinne können Strategien der Visualisierung auch als Distinktionsmittel beschrieben werden, das bestimmten Fachgruppen die 
Expertise zuschreibt, bestimmte Praktiken zu verfolgen. Außerdem spricht der Einsatz von Strategien der Visualisierung und Techniken der Inszenierung für die Objektivierung »der Jugend«, die von der Partizipation am Programm ausgeschlossen ist und auf deren Marginalisierung Jugendgewaltprävention letztlich abzielt.

\subsubsection{Zusammenfassung}

Im Kontext des »Nationalen Präventionsprogramms Jugend und Gewalt« wird Jugendgewalt als Deutungsmuster rekonstruiert, indem auf Strategien der Normalisierung von Jugendgewalt, auf Praktiken der Inszenierung und Visualisierung sowie auf den Gebrauch utopischer und dystopischer Szenarien zurückgegriffen wird. Mittels dieser Strategien wird die Bedrohung der eigenen, friedlichen Gesellschaft konstruiert und, damit verbunden, an deren Sicherheitsbedürfnis appelliert sowie daran, dass diesem nur gemeinsam entgegengekommen werden kann. Dabei wird differenziert zwischen »normaler« Jugendgewalt, die nicht kontingent ist, und »pathologischer« Jugendgewalt, die ein »friedliches« Kollektiv bedroht. Die Ziele des Programms betreffen nun nicht primär den Schutz vor bedrohlichen Formen von Jugendgewalt bzw. Jugendlichen, sondern vielmehr die Möglichkeit, »normale« Formen von Jugendgewalt dauerhaft bearbeitbar zu machen, womit eine bestehende Infrastruktur aufrechterhalten werden soll. Der Diskurs über Jugendgewaltprävention ist somit nicht nur als hoch normativ und ausschließend, sondern auch als in hohem Maße selbstreferenziell zu bewerten. Die Interviews sind dahingehen interpretiert worden, was im Sprechen über die jeweiligen Fachbereiche in welcher Weise problematisiert wird, welche Präventionsverständnisse und -konzepte daran anschließen und wie diese begründet werden. Ein zentrales Ergebnis betrifft dabei die Brüche zwischen den jeweils aus der eigenen Expertise heraus entwickelten Relevanzen (Innenorientierungen) und den von außen adressierten Erwartungen (Außenorientierungen). Diese Interpretationen stehen im Kontext zum »Nationalen Präventionsprogramm Jugend und Gewalt«. Die befragten Fachpersonen, insbesondere im Kontext offener und aufsuchender Jugendarbeit, stehen mit ihrem grundlegenden Verständnis von Prävention sowie ihren Präventionskonzepten tendenziell in einem ambivalenten Verhältnis zu kriminalpolitischer Programmatik. Dies lässt sich v.a. daran beobachten, dass sie Aufträge im Sinne einer an Risiken, Wirksamkeit und Evidenz als orientierte Prävention, die eine Zusammenarbeit mit anderen Fachbereichen und Professionen einfordert, durchaus explizieren und implizieren und sich dazu angehalten sehen, sich dazu zu positionieren. Diese Orientierung widerspricht jedoch eigenen Relevanzsetzungen und fachbereichsspezifischen Erfordernissen hinsichtlich der Jugendgewaltprävention. Die Fachpersonen situieren sich hier in einem Spannungsfeld, das sich durch 
verschiedene Ambivalenzen auszeichnet. Sie positionieren sich zwischen miteinander konkurrierenden Ansprüchen hinsichtlich Prävention und Intervention. Die Arbeit mit Jugendlichen ist durch Spannungsverhältnisse zwischen Prävention im Sinne »sozialer Prävention«, die aus einem Schutzbedürfnis der Jugendlichen resultiert, und Intervention, die aus einem Kontrollbedürfnis resultiert, charakterisiert. Dieses Spannungsverhältnis, das die alltägliche Arbeit auszeichnet, wird zudem aufgespannt durch ein, so hier die Annahme, an kriminalpolitischer Programmatik orientiertes Verständnis von Prävention, das quer zu den je eigenen Verständnissen von Prävention und den daran entwickelten Konzepten von Prävention und Intervention liegt. Die Fachpersonen werden bezüglich ihrer kollektiven Verantwortung angerufen und readressieren diese wiederum an Jugendliche, indem sie sich an der kriminalpolitischen Programmatik orientieren. In dieser Weise (re-)adressieren sie Jugendliche in ihrer Verantwortlichkeit, ein »gutes« Leben zu führen oder, wie Herr Tanner es formuliert, ihr »Leben erfolgreich zu gestalten «. Dieser Transformationsprozess lässt sich als »Übertragung von Verantwortung (Responsibilisierung)《 (Oelkers 2013: 163) oder mit anderen Worten als Prozess der Überantwortung denken. In den Adressierungsweisen des »Nationalen Präventionsprogramms Jugend und Gewalt« wird kollektive Betroffenheit und, damit verbunden, kollektive Verantwortung konstruiert, die in den Konferenzen reproduziert wird. Diese Kollektivierung vollzieht sich insbesondere über (normative) Verweise auf eine gemeinsame Wissensbasis, eine dauerhafte Bedrohungslage und einen daran anknüpfenden Appell an ein an gemeinsamen Interessen orientiertes und auf die Zukunft gerichtetes Handeln. Die Metapher, dass alle im selben Boot sitzen, vermittelt eine gemeinsame Bedrohung, der nur gemeinsam entgegengewirkt werden kann, und zwar im Rahmen einer Standardisierung sog. »evidenzbasierter« Prävention. Einzelkämpfer sollten nicht unterstützt werden. Die Konstruktion des Kollektivs wird in Abgrenzung einer friedlichen Gesellschaft von »den Jugendlichen« bzw. »der Jugend « verfestigt, wobei zwischen »normaler u und »pathologischer « Jugend differenziert und auf utopische und dystopische Szenarien zurückgegriffen wird: Eine "gewalttätige« bzw. »gewaltaffine« Minderheit Jugendlicher, bedroht eine »friedliche« Gesellschaft und sichert gleichzeitig die Existenz betroffener Professionen. Demnach zielt das Präventionsprogramm weniger auf »Bekämpfung der Jugendgewalt« als vielmehr auf Bestandssicherung im Sinne der Sicherung des Status quo und v.a. der etablierten »Professionen «, indem es Jugendgewalt als dauerhafte Bedrohung rekonstruiert. Damit ist es als hoch selbstreferenziell anzusehen. Bemerkenswert ist dabei, dass es nicht primär Jugendgewalt im Sinne jugendlichen Gewalthandelns problematisiert, wie die Fachpersonen es in ihrem eigenen Selbstverständnis tun, sondern die soziale Gruppe der Jugendlichen. Jugendliche werden so zu Objekten des Diskurses, was durch den Einsatz von Strategien der Visualisierung und Techniken der Inszenierung 
weiter verstärkt wird. Der Gewaltbegriff fungiert dabei als Sinnleerformel, die es ermöglicht, jegliche Phänomene unter diesem zu subsummieren und den Verantwortungsbereich auf Seiten der Fachpersonen möglichst weit auszudehnen. Die soziale Gruppe der Jugendlichen wird in diesem Prozess dauerhaft als gefährdete und gefährliche Gruppe und somit als Gegenstand politischer Problemdiskurse und Sozialer Arbeit zuschreibbar gemacht.

\subsection{Zusammenfassung Und Integration der Ergebnisse}

In Bezug auf die Forschungsfrage lässt sich zusammenfassend feststellen, dass die befragten Fachpersonen sich hinsichtlich ihrer Problematisierungsweisen (von Jugendgewalt), der daran anschließenden Präventionsverständnisse und -konzepte sowie deren Begründungsweisen in einer hoch ambivalenten Position zwischen Innen- und Außenorientierungen situieren:

Innenorientierungen: Einerseits distanzieren sich die befragten Fachpersonen explizit und implizit von kriminalpolitischer Programmatik:

- Sie distanzieren sich aus einer gesellschaftskritischen Positionierung heraus von Prozessen gesellschaftlicher, politischer und medialer Problematisierung und Skandalisierung von Jugendgewalt. Jugendgewalt sei v.a. eine Konsequenz sozialstrukturell bedingter Ungerechtigkeiten.

- Sie beschreiben Jugendgewalt primär als problematisches Handeln, das sie weniger auf die soziale Gruppe der Jugendlichen projizieren, als das Programm es tut. Dies erfordert unter Umständen Intervention. Das Ausmaß bzw. die Qualität problematischen Handelns zu beurteilen und sich, damit verbunden, für oder gegen Intervention zu entscheiden, sehen sie als große Herausforderung an, die oft nicht an der konkreten Situation, sondern erst retrospektiv erfolgt.

- Die Fachpersonen orientieren sich an zwei unterschiedlichen Präventionsverständnissen, die sie nur schwer miteinander vereinbaren können: Einem auf Wirksamkeit und Evidenz abzielenden Verständnis von Prävention stehen "ganzheitliche«, am individuellen Fall orientierte, Herangehensweisen gegenüber, etwa der Anspruch, individuelle Entwicklungsprozesse zu rahmen.

- Sie sehen Jugendgewaltprävention als weniger zentral für ihre jeweiligen Fachbereiche an als Intervention, wobei sie Prävention und Intervention nicht strikt trennen können.

- Die Unvereinbarkeit der aus den eigenen Handlungskontexten heraus entwickelten Problematisierungsweisen und Präventionsverständnisse und -konzepte mit denen von außen an sie herangetragenen stellt sich als Di- 
lemma dar, das nicht ohne Weiteres zu lösen ist und Stigmatisierungs- und Ausschließungsprozesse begünstigt.

Außenorientierungen: Andererseits sprechen die Ergebnisse für eine Orientierung der befragten Fachpersonen an kriminalpolitischer Programmatik:

- Aus einer gesellschaftskritischen Positionierung heraus entwickeln die befragten Fachpersonen eine defizitorientierte Perspektive auf Jugendliche. Jugendliche seien potenzielle Täter und »Opfer« von Jugendgewalt sowie als Opfer gesellschaftlicher Skandalisierungsprozesse anzusehen. Diese Zuschreibungen werden über biologisch und kulturell bedingte Schwierigkeiten hinsichtlich der Artikulationsfähigkeit, der Einsichtigkeit und des Bildungsmangels Jugendlicher begründet. Die Fachpersonen setzen in ihren Konzepten jedoch nicht an gesellschaftlichen Strukturen, sondern an den Jugendlichen an. Die aus diesem Deutungshintergrund heraus entwickelten Präventionsverständnisse und -konzepte zielen auf die Anpassung Jugendlicher an gesellschaftliche Strukturen und die normativen Erwartungen der »Mehrheitsgesellschaft«ab.

- In diesem Zusammenhang normalisieren sie Jugendgewalt im Sinne jugendlichen Gewalthandelns in hohem Maße. Als historisches, natürliches, biologisches und kulturelles Konstrukt dient es der Aufrechterhaltung und Legitimation der »Professionen«, die mit Jugendgewaltprävention betraut sind, und damit letztlich auch der Generationenverhältnisse.

- Über die Normalisierung von Jugendgewalt konstruieren sie normative Erwartungen bezüglich der sozialen Gruppe der Jugendlichen in Abgrenzung zu den Erwachsenen. Dabei individualisieren sie Risiken insofern, als sie die Normalisierung von Jugendgewalt im Sinne jugendlichen Gewaltverhaltens verallgemeinern. In den Interviews spiegelt sich eine Differenzierung zwischen »normal« und »pathologisch « wider, die sich im Gegensatz zum Programm nicht primär auf die Jugend bezieht, sondern auf jugendliches Gewalthandeln. Diese Differenzierung können die Fachpersonen jedoch nicht zum Gegenstand von Intervention und Prävention machen (Distanzierung). Die in diesem Zusammenhang beobachtete defizitorientierte Perspektive auf Jugendliche kann als eine Grundhaltung der hier befragten Fachpersonen angesehen werden und wird von ihnen verallgemeinert und individualisiert.

Die den Fachpersonen zugeschriebene Verantwortung und Verantwortlichkeit für Jugendgewaltprävention, so die These, wird aus diesem Spannungsverhältnis heraus auf Jugendliche übertragen - an diese »überantwortet«. Die normative Erwartung, »gute«, »beste« oder »erfolgversprechende« Prävention zu realisieren, wird (re-)adressiert an die Jugendlichen über die an sie gerichtete 
Erwartung, »gut« zu sein, »vorzüglich « zu sein sowie ein »gutes « und »erfolgreiches« Leben $\mathrm{zu}$ führen. Aktivierungspolitisch gelesen, fungiert Jugendgewaltprävention als Instrument einer Aktivierungspolitik, die nicht nur auf die Professionen Sozialer Arbeit abzielt, sondern auch auf Jugendliche bzw. Klientinnen und Klienten Sozialer Arbeit im Allgemeinen. Die Fachpersonen im Kontext von Jugendgewaltprävention werden dabei zu Subjekten, die Jugendlichen zu Objekten des Diskurses. In der Perspektive der Fachpersonen herrschen Deutungsmuster von Jugendgewaltprävention vor, die nicht oder nur schwer miteinander zu vereinbaren sind und ein Dilemma beschreiben: Prävention als Konzept, die positive Entwicklung Jugendlicher zu rahmen (»Innen«), steht der Deutung von Jugendgewaltprävention als nachhaltiges, evidentes und in seiner Wirksamkeit messbares Konstrukt gegenüber (»Außen«). Erstere Deutung kann als Grundhaltung der Fachpersonen und weniger als expliziter Gegenstandsbereich der jeweiligen Fachbereiche verstanden werden. So gesehen ist Prävention nicht messbar, sondern nur am Fall und retrospektiv zu beurteilen. Konkrete »Jugendgewaltpräventionsaufträge«, Erfolgsruck und die Verkennung der eigenen Präventionsarbeit stehen den eigenen Präventionskonzepten gegenüber und verkomplizieren das Spannungsfeld zwischen Prävention und Intervention zusätzlich. Die Beobachtung, dass die Fachpersonen Jugendliche hinsichtlich ihrer Verantwortung für Prävention zu aktivieren suchen, wird in der hier vorliegenden Arbeit als Strategie interpretiert, diesem Dilemma etwas entgegenzusetzen - dieses zu entspannen. In der Orientierung der Fachpersonen an kriminalpolitischer Programmatik, so die zentrale These, werden Ziele und Ergebnisse von Jugendgewaltprävention, welche die Fachpersonen im Rahmen ihrer Handlungskontexte an Jugendliche projizieren, an diese überantwortet.

\section{Zusammenhang zwischen Normalisierung von Jugendgewalt und Prävention aus der Sicht der befragten Fachpersonen}

Aus den Interviews mit den Fachpersonen geht eine defizitorientierte Perspektive auf Jugendliche hervor, vor deren Hintergrund sie Jugendgewalt im Sinne individuellen Gewalthandelns normalisieren. Die Defizite führen sie dabei nicht auf elterliche Erziehungspraktiken zurück, wie Dollinger und SchmidtSemisch allgemein für kriminalpräventive Kontexte beobachten (Dollinger/ Schmidt-Semisch 2011: 14), sondern kann als grundlegendes hegemoniales Verhältnis von Kultur und Natur gedeutet werden. Aus soziologischer Perspektive ist demnach weniger das Verhältnis verschiedener Institutionen der Bildung und Erziehung zu problematisieren, sondern das hegemoniale Verhältnis von Individuum und Gesellschaft, das kulturalisierenden, historisierenden, biologisierenden und naturalisierenden und in diesem Sinne letztlich normativen Deutungsmustern unterliegt. An eine Problematisierung jugendlichen Gewalthandelns in dem Sinne, dass es neben »normalen« Formen auch »pathologische 
Formen« gebe, schließen die Fachpersonen in ihren Präventionsverständnissen und -konzepten jedoch nicht an, da sie diese Unterscheidung in der jeweiligen Situation meist nicht treffen können. Vielmehr beziehen die Fachpersonen Prävention im Kontext Sozialer Arbeit auf jugendliche Handlungsweisen im Sinne einer »Sozialen Prävention« (Reder/Ziegler 2011), von der Intervention nicht klar abgrenzbar ist. Die befragten Fachpersonen haben kein konkretes Konzept von Jugendgewaltprävention, sondern ein an »individuellen Lebenslagen« orientiertes Verständnis von Prävention, das »der je individuellen Lebenslage und der Besonderheiten der alltäglichen Lebensführung der Betroffenen gerecht « (ebd.: 367 ) wird. So zielen die Konzepte jedoch nicht nur darauf ab, Jugendlichen durch bestimmte Rahmenbedingungen Möglichkeiten des Lernens, des Kompetenzerwerbs, der Bildung und insgesamt der persönlichen Entwicklung zuzugestehen und sie bei der Gestaltung individueller Lebensläufe zu unterstützen, sondern vermitteln auch normative Erwartungen darüber, welche Ergebnisse die Jugendlichen dabei erzielen sollen. So betont Herr Tanner, das Ziel von Prävention sei letztlich die »erfolgreiche Lebensgestaltung«, was er am Beispiel des Postboten darstellt. Es zeigt sich insbesondere auch an den normativen Erwartungen Frau Blaums an den Jugendlichen, sich selbstständig weiterzubilden. Sie erwartet nicht nur, dass er sich weiterbildet, sondern dass er sich auf eine bestimmte Art und Weise und mit einer bestimmten Einsicht weiterbildet. Diese Erwartungen brechen mit einer gesellschaftskritischen Positionierung der Fachpersonen. So sehen sie Jugendliche als »Opfer« gesellschaftlicher Verhältnisse an, insofern als sie sich aufgrund ihrer altersbedingten fehlenden Kompetenzen und Bildungsdefizite nicht gegen Diskriminierungsprozesse zur Wehr setzen könnten. Die »Überantwortung« von Prävention an Jugendliche ist jedoch als Anpassung an gesellschaftliche Verhältnisse anzusehen und wird, wie das Beispiel der Erzählung Frau Blaums transparent macht, in hohem Grade individualisierbar. Hier stellt sich die Frage nach dem Verhältnis von Gesellschaftskritik und Sozialer Arbeit, die im Kapitel 5 vertieft wird. So gesehen verfolgen insbesondere die Fachpersonen aus der offenen Jugendarbeit nicht nur das Ziel, Lern- und Bildungserfahrungen Jugendlicher zu rahmen und zu ermöglichen, also »eine möglichst offene, wenig strukturierte, an den Interessen der Jugendlichen ansetzende Arbeit« (Huber 2014: 42) zu leisten, die sich an der Lösung individueller Problemlagen orientiert, sondern sie folgen gleichzeitig einer sozialpolitischen Aktivierungslogik, indem sie eine »erfolgreiche Lebensgestaltung « als Ziel und Norm setzen und es letztlich in die Verantwortung der Jugendlichen selbst stellen, diesen Anpassungsprozess an die Gesellschaft in »erfolgreicher« Weise zu vollziehen. Dies verweist auf ein grundlegendes Dilemma von Kinder- und Jugendarbeit (vgl. Jordan/Maykus/Stuckstätte 2015: 147f.), das jedoch im Kontext von Jugendgewaltprävention noch nicht expliziert worden ist. Herr Tanner und Frau Blaum explizieren in ihren Darstellungen der für die offene Jugendarbeit spezifischen Aufgaben 
eigentlich grundlegende rechtliche Orientierungen der Kinder- und Jugendarbeit, etwa »Offenheit«, »Marginalität« und »Diskursivität« (ebd.: 245f.), und entwickeln daran auch ihre eigene Expertise. Jedoch verweisen sie, wie auch andere Fachpersonen, in Bezug auf Prävention gleichzeitig auf einen hohen Erwartungsdruck von außen, der ihr eigentliches Selbstverständnis torpediert.

"Grundsätzlich kann festgestellt werden, dass Jugendarbeit als kommunale Aufgabe in einem besonderen Spannungsverhältnis steht: Einerseits bekommt sie die praktischen Probleme und politischen Implikationen der Arbeit mit Jugendlichen am unmittelbarsten zu spüren, andererseits wirken sich administrativ-bürokratische Regelungen und der öffentliche Legitimationsdruck auf sie am stärksten aus." (Ebd.: 147)

Dieses »besondere Spannungsverhältnis« wird insbesondere dadurch befördert, dass Politik und Öffentlichkeit Prävention eine so hohe Bedeutung zuschreiben, zur Kernaufgabe der Jugendarbeit schlechthin erheben und diesbezügliche Erfolge einfordern. Das Konzept »Jugend «, das auch in der wissenschaftlichen Tradition sowie in medialen und politischen Darstellungsweisen im Sinne einer Naturalisierung von Defiziten, aus der Gewalthandeln erklärt wird, negativ konnotiert ist (vgl. Anhorn 2002: 48f.; Bettinger 2007: 83), wird auf der Darstellungsebene der Erzählung und Beschreibung der Praxis offener Jugendarbeit individualisiert, wobei auch auf Risiken zurückgegriffen wird. Dies kann als zu kriminalpolitischer Programmatik »konform« angesehen werden. Denn

"kennzeichnend für den versicherungsmathematischen Risikomanagerialismus sind eine deutliche Tendenz zur individualisierten Verantwortlichmachung der Betroffenen, eine Betonung von Frühinterventionen sowie die Bereitschaft auf durchaus rigorose bis punitive Interventionsmuster zurückzugreifen, wenn die Betroffenen nicht die als notwendig erachtete Kooperationsbereitschaft zeigen“ (Reder/Ziegler 2011: 367).

Risiko wird so zu einer individuellen Zuschreibung, die auch individuell zu verantworten ist (vgl. Cremer-Schäfer 2016: 20).

\section{Jugendgewaltprävention als Strategie der "Bestandssicherung" auf der Ebene kriminalpolitischer Programmatik}

Wie legitimiert sich Jugendgewaltprävention, wenn jugendliches Gewalthandeln aus einer Defizitperspektive heraus normalisiert wird, "pathologische« Formen davon nur einen kleinen Teil der Jugendlichen betreffen und die Polizeistatistik rückgängige Deliktquoten beschreibt? Mit der Frage der Begründung sind auch Fragen nach dem Verständnis von Jugendgewaltprävention, nach »Durchführungsweisen« sowie nach Zielen bzw. Ergebnissen verbunden. Das Programm differenziert primär zwischen »normaler« und »patho- 
logischer« Jugendgewalt, wobei es auch hier das dieser Gruppe zugeschriebene Gewalthandeln normalisiert und historisiert. Dabei konstruiert es ein Kollektiv, das als Teil einer »friedlichen « Gesellschaft, die eigentlich über eine »gute Jugend «, gar »hervorragende« Jugend verfügt, durch eine kleine Gruppe »schwarzer Schafe« bedroht wird. In der Konstruktion von Bedrohungsszenarien greift das Programm also auf utopische und dystopische Szenarien zurück. Schreiber zeigt in ihrer diskurstheoretisch versierten Analyse kriminalpräventiver Bestrebungen in Deutschland, dass diese insbesondere in utopischen wie dystopischen Visionen Legitimierung suchen, insofern als somit die Notwendigkeit von Prävention aufrechterhalten bleibt, weil sie nie vollendet sein kann (vgl. Schreiber 2011: 145f.). Die Utopie, die ihm Rahmen des »Nationalen Präventionsprogramms Jugend und Gewalt« entfaltet wird, bezieht sich auf den Ist-Zustand der Gesellschaft: Alles soll so bleiben, wie es ist. Ginge die Rate der Jugendgewalt drastisch zurück, wären der Präventionsapparat und seine »Professionen « gefährdet. So erklärt sich auch die Normalisierung von Jugendgewalt, die im Rückgriff auf historisierende, biologisierende, kulturalisierende und naturalisierende Deutungsmuster entfaltet wird, weil sie die Bestrebung plausibilisiert, Jugendgewaltprävention als dauerhaftes Thema politisch zu institutionalisieren, woran auch die befragten Fachpersonen anschließen. Dabei hat das Bild, dass alle, insbesondere Bund, Kantone, Städte und Gemeinden, gemeinsam im selben Boot sitzen, eine zentrale Bedeutung. Bedroht wird dieses Szenario nicht nur durch den »Einzelkämpfer«, der im »Kampf« gegen Jugendgewalt den kollektiven Interessen entgegensteuern könnte, sondern auch durch Massen von Jugendlichen, die nicht mehr identifiziert und kontrolliert werden können. Auch die Verweise auf die Opfer und die horrenden Kosten, die mit der lebenslangen Unterbringung und Therapie von Tätern einhergehen, sind selbst dystopische Momente des »Nationalen Präventionsprogramms Jugend und Gewalt«, die auch Schreiber bezüglich kommunalter Gewaltprävention beobachtet (vgl. ebd.: 168f.). Die Kosten, die Straftäter dem Staat verursachen, werden insgesamt höher eingeschätzt als die Kosten, die Prävention verursacht, wobei implizit davon ausgegangen wird, dass Prävention wirkt. »Erfolge«, die sich in der Statistik als Rückgang von zur Anzeige gebrachter Gewalt zeigen, werden auf das Programm zurückgeführt. Damit wird weiterhin an das Kollektiv appelliert, auf dem »richtigen Kurs « zu bleiben. Jugendgewaltprävention objektiviert sowohl Jugend als auch bestimmte Risikogruppen Jugendlicher, die das Kollektiv, die (noch) »friedliche« Gesellschaft, bedrohen. Die Orientierung des Programms an Risikopopulationen kann damit als eine Dekonstruktion des Subjekts verstanden werden (vgl. Schreiber 2011: 149), die beispielsweise Frau Blaum reproduziert, indem sie Risiken individualisiert. Somit wird sowohl Jugend als auch eine bestimmte Gruppe Jugendlicher als Risikopopulation und damit als Zielgruppe präventiver Maßnahmen konstruiert und kontextualisiert. Das Programm zielt in seinem Präventionsver- 
ständnis primär auf Bestandssicherung, insbesondere auf die Sicherung der »Professionen« sowie der generationalen Ordnung im Allgemeinen, und ruft dabei die Fachpersonen in ihrer Subjektivität als verantwortliche Akteure an. Aus dieser Perspektive kann es nicht Ziel von Jugendgewaltprävention sein, den Bestand der »schwarzen Schafe « zu verringern, denn dies würde letztlich den Präventionsapparat und seine »Professionen« bedrohen. Auch Schreiber beobachtet in ihrer Analyse kommunaler Kriminalprävention, dass mit

"der Prävention eine spezifische Bezugnahme auf das Subjekt einhergeht. Prävention entwickelt inr Wissen nicht vorrangig in der Auseinandersetzung mit Individuen, sondern aus statistischen Korrelationen. Kriminologische Vorstellungen vom Individuum als Träger einer Bedrohung werden abgelöst durch Populationen, die aus der Kombination abstrakter, generell risikoträchtiger Faktoren geschaffen und gesteuert werden. [...] Als Steuerungsinstrument ist sie [die Prävention] gar nicht bestrebt, Kriminalität gänzlich abzuschaffen. Vielmehr versucht sie, die Kriminalität und ihre Wahrnehmung in einem für das Funktionieren der Gesamtbevölkerung erträglichen Maß zu halten. Bevölkerung durch Prävention verwalten meint dann, die zuvor durch Konvention bestimmten Normgrößen einzuhalten.“ (Ebd.: 151f.)

In diesem Präventionsverständnis appelliert das Programm an die Fachpersonen in ihrer kollektiven Verantwortung (die schließlich individualisiert wird) und der Notwendigkeit, in Bezug auf »erfolgversprechende« Prävention gemeinsam zu handeln. Jugendgewalt wird dabei normalisiert und rekonstruiert und damit für daran anknüpfende Handlungskontexte reproduzierbar. Aus der Perspektive der befragten Fachpersonen bedarf Prävention keiner Legitimation in diesem Sinne. Prävention im Sinne »sozialer Prävention« ist selbstverständliches Leitbild und Orientierungsrahmen, jedoch nicht im Anschluss an eine Problematisierung von Jugendgewalt im Sinne jugendlichen Gewalthandelns. Jugendliches Gewalthandeln in normalem Maß ist ein alltägliches Phänomen, auf das die Fachpersonen nur interventiv reagieren können. Jugendliches Gewalthandeln »pathologischer« Art bezieht sich hingegen in erster Linie auf die Gefährdung individueller Biografien, was Jugendgewaltprävention somit als Gegenstand der jeweiligen Handlungskontexte weiter ausschließt, da die Fachpersonen nicht über den Zugang zu den relevanten Informationen über individuelle Jugendliche und deren Lebenswelten verfügen. Durch eine zusätzliche Belastung dieses Spannungsverhältnisses von Prävention und Intervention werden Jugendliche bezüglich Prävention im Sinne einer »Sozialen Prävention « allerdings in ihrer Verantwortlichkeit (re-)adressiert. Prävention zielt auf die Verantwortlichkeit Jugendlicher ab, eine individuelle Anpassungsleistung an gesellschaftliche Strukturen zu vollziehen, was jedoch nicht als Prozess beobachtbar ist, sondern nur retrospektiv als solche erkannt und beurteilt werden kann. Prävention beschreibt also die Art und Weise wie die »kontrollierte« Er- 
möglichung von Lern- und Bildungsprozessen sowie des Kompetenzerwerbs vonstattengehen kann, zielt jedoch letztlich auf individuelle Anpassung. Die Normativität, die hinter dieser Adressierung steht, spitzt sich im Konzept der »erfolgreichen Lebensgestaltung « zu, das einen Höhepunkt in der Aktivierung (vgl. Kessl/Krasmann 2005: 237) von Adressatinnen und Adressaten Sozialer Arbeit darstellt. Zwar sind Normativität und Soziale Arbeit grundlegend aneinandergekoppelt, insofern als »normativer Bezugspunkt professionellen Handelns eine Orientierung an der Herstellung individueller Handlungskompetenz, sozialer Gerechtigkeit und Bildung über die Schaffung individueller Entwicklungsbedingungen ist.« (Groenemeyer 2015: 33). Dieser Bezugspunkt orientiert sich in den Darstellungsweisen der hier befragten Fachpersonen jedoch nicht am Fall in einer konkreten Situation, sondern an der möglichen Zukunft eines »Falles« im Erwachsenenalter. Die individuelle Handlungskompetenz Jugendlicher soll nicht in situ hergestellt werden, sondern fokussiert auf die Norm eines »guten« Lebens im Erwachsenenalter. Gleichzeitig orientieren sich die Fachpersonen an Prävention als Leitbild, unter dem ein nachhaltiger, wirksamer und evidenter Prozess zu verstehen ist, den sie zwar nicht einlösen können, jedoch als Erfolgsdruck wahrnehmen und den sie über Erfolgsgeschichten darstellen, etwa in der Erzählung Herrn Tanners bezüglich der erfolgreichen Lebensgestaltung seines Postboten, die nicht ohne Verwunderung auskommt. ${ }^{5}$ Auch wenn sich die Fachpersonen selbst als wirksam erleben, entspricht dies nicht unbedingt den Erfahrungen der Öffentlichkeit, die Erwartungen formuliert, welche die Fachpersonen nicht erfüllen können. So erlebt Herr Tanner die aufsuchende Jugendarbeit seiner Organisation als gelungene Prävention, weil er selbst eine Verbesserung hinsichtlich der Nutzung öffentlicher Räume wahrnimmt. Seine Wahrnehmung wird jedoch nicht von anderen beteiligten Akteuren geteilt, welche die Verbesserung auf polizeiliche Präsenz zurückführen. Die Verkennung der Erfolge von Jugendarbeit stellt eine massive Bedrohung dar. So stellt sich auch Frau Blaum angesichts eines nicht erfüllbaren Präventionsauftrags im Sinne einer wirksamen und nachhaltigen Intervention in ihrer Handlungswirksamkeit als stark eingeschränkt dar. Soziale Arbeit läuft, so plausibilisieren die Ergebnisse der vorliegenden Arbeit, also nicht nur Gefahr, durch Orientierung an ordnungspolitischen Diskursen ihre Autonomie zu verlieren und selbst zu kriminalisieren, sondern auch sich selbst nicht als wirksam oder gar ohnmächtig zu erleben (vgl. Anhorn/Bettinger 2002: 223).

5 | Der Verweis auf Erfolge und das "Breittreten" (Herr Gold) dieser nimmt in den meisten der Interviews einen großen Raum ein, was aber zum Teil auch reflektiert wird, weil es für das Bestehen der eigenen "Profession " als notwendig erachtet wird. So sieht Herr Unterwasser die mediale Inszenierung eines erfolgreichen Falles zwar kritisch, gleichzeitig aber auch als nützlich an. 


\subsection{Methodische Reflexion}

Die hier vorliegende Arbeit steht in einem Spannungsverhältnis zwischen dem Anspruch, die unterschiedlichen Datenkorpora zu verknüpfen und die jeweils sehr reichhaltigen Datenkorpora für sich einer intensiven Analyse zu unterziehen. Grundsätzlich stehen die Datenkorpora für sich. Die Interviews orientieren sich an einem Leitfaden, der sich nicht direkt auf das »Nationale Präventionsprogramm Jugend und Gewalt« bezieht, jedoch gebraucht die Interviewerin Wissen, das in diesem Zusammenhang relevant ist, etwa bezüglich der Problematisierung von Jugendgewalt, »guter« Prävention und betroffener Adressatinnen und Adressaten von Maßnahmen im Kontext von Jugendgewaltprävention. Auch die befragten Fachpersonen nehmen auf dieses Wissen Bezug, grenzen sich aber auch davon ab und positionieren sich nur mit wenigen Ausnahmen explizit zum Programm. Jedoch lassen sich mit dem Subjekt als analytische Kategorie die beiden Ebenen des Sprechens über Jugendgewaltprävention durchaus zusammen denken und aufeinander beziehen. Eine Distanzierung zu kriminalpolitischer Programmatik lässt sich insbesondere in den Einstiegspassagen der Interviews beobachten, wo sich die Befragten kritisch mit den Adressierungsweisen der Interviewerin auseinandersetzen. Diese Auseinandersetzung zeigt sich sowohl auf der Gesprächsebene, etwa an der Ironisierung der Fragen bzw. der ihnen zugeschrieben Bedeutungen oder der Umformulierung der Fragen, als auch auf inhaltlicher Ebene. Sie zeigt sich an einer gesellschaftskritischen Positionierung, die in Abgrenzung von Problemdiskursen entwickelt wird, und darin, dass die Fachpersonen angeben, die ihnen von außen zugeschriebenen Erwartungen hinsichtlich Jugendgewaltprävention nicht erfüllen zu können. Im Verlauf der Interviews wechseln die Fachpersonen ihre Positionierungen, was auch im Zusammenhang mit den Zuschreibungen durch die Interviewerin steht. Die Beobachtung der Relativierung ist also zentral, weil sie im Zusammenhang mit der Beobachtung steht, dass die Fachpersonen Jugendgewaltprävention nicht als wesentlichen Teil ihrer jeweiligen Fachbereiche und Expertisen ansehen. Das lässt darauf schließen, dass die mit der als »(un-)doing expertise « bezeichnete Strategie nicht nur der Interviewform geschuldet ist, sondern im Kontext der Jugendgewaltprävention zu lesen ist. Außerdem fällt auf, dass die meisten der befragten Fachpersonen ihre eigene Expertise nicht allein aus ihren Berufsbiografien und ihren Handlungskontexten heraus entwickeln, sondern auch andere lebensweltliche Erfahrungen, etwa mit den eigenen Kindern, Familienangehörigen oder sonstigen Schlüsselerlebnissen, mit einfließen lassen. In dieser Hinsicht zeichnen sich die Interviews durch einen häufig wiederholten Wechsel von Positionierungen aus, der als Distanzierung von einer einseitigen Adressierung als Expertin bzw. Experte für Jugendgewaltprävention durch die Interviewerin gedeutet werden kann und auch auf Ambivalenzen hinweist, die aus dem Be- 
streben, unterschiedlichen Erwartungen gerecht zu werden, resultieren. Es ist davon auszugehen, dass nicht nur die (Einstiegs-)Fragen im Interviewverlauf, sondern bereits das Anschreiben relevante Zuschreibungen enthält. Beispielsweise ist Herr Tanner sogar schriftlich auf bestimmte Inhalte vorbereitet und zeigt an, mit bestimmten Fragen gerechnet zu haben. Auch Herr Vonderstatt hat bereits seine eigenen Relevanzen gesetzt, insofern zu beobachten ist, dass er die Fragen der Interviewerin gar nicht beantwortet, sondern umformuliert und letztlich seine eigenen Fragen beantwortet. Möglicherweise werden die durch das Anschreiben erweckten Erwartungen der Befragten durch die zunächst gewählte Einstiegsfrage befördert. Diese Frage verbinden die Fachpersonen nicht mit ihren eigenen Expertisen: Die Erfahrungen mit Jugendgewalt begrenzen sich hier nicht auf die Fachbereiche, sondern auf unterschiedlichste Lebenswelten. Die Interviews, die mit einer offenen Erzählaufforderung seitens der Interviewerin eingeleitet werden, lassen mehr Offenheit zu, sodass die Befragten sich zu erwarteten Erwartungen positionieren und eigene Relevanzsetzungen entwickeln können. Das zeigt sich beispielsweise an dem Interview mit Frau Schock, die sich eben nicht primär über ihr Pilotprojekt im Kontext von Jugendgewaltprävention, sondern über ihre Arbeit als Familientherapeutin identifiziert. Daraus kann geschlossen werden, dass sich das Subjekt nicht allein über Anrufungsprozesse konstituiert, sondern auch über Erfahrungen, die jedoch in anderen Kontexten relevant sind und wiederum als Teil eines Diskurses bzw. als Diskursfragmente angesehen werden können. So kommen die Fachpersonen im Kontext des Programms in ihrer Verantwortung und Verantwortlichkeit als Subjekte eines bestimmten Diskurszusammenhangs, der Prävention, Aktivierung und Sicherheit miteinander verknüpft, in den Blick. Die Außenorientierungen der Fachpersonen beziehen sich auf das für diesen Diskurszusammenhang relevante Wissen, insbesondere die Durchführungsweisen und Ziele von Jugendgewaltprävention, und brechen mit deren Innenorientierungen. Diese Außenorientierungen kommen als Erwartungen in den Blick, die von außen an die Fachpersonen herangetragen werden, und werden hier als Orientierungen im Sinne eines kollektiv geteilten Deutungsrahmens an kriminalpolitischer Programmatik bezeichnet. Das Subjekt fungiert in der vorliegenden Arbeit also als analytisches Bindeglied. Des Weiteren ermöglicht die subjekttheoretische Perspektive auf Jugendgewaltprävention, in diesem Kontext angesiedelte Prozesse der Objektivierung in den Blick zu nehmen. Zudem ist anzumerken, dass im Rahmen dieser Arbeit nicht Praktiken in den Blick kommen, sondern das Sprechen über Praktiken im Kontext von Jugendgewaltprävention, das Aufschluss über Orientierungsweisen in diesem Kontext gibt. Insofern sich die Fachpersonen in ihren Orientierungen auf Wissen beziehen, das diskursiv vermittelt ist, wird hier allgemein von »Praktiken des Wissens« und spezifisch vom »Gebrauch von Prävention« gesprochen. Präven- 
tion bzw. Jugendgewaltprävention als Praktik ist im Rahmen der vorliegenden Arbeit nicht sichtbar:

"Das 'praktische Wissen', das in einer sozialen Praktik mobilisiert wird und das die Praxistheorie rekonstruieren will, umfasst dabei verschiedene Elemente: ein Wissen im Sinne eines interpretativen Verstehens, d.h. einer routinemäßigen Zuschreibung von Bedeutungen zu Gegenständen, Personen, abstrakten Entitäten, dem reigenen Selbst etc.; ein i.e.S. methodisches Wissen, d.h. script-förmige Prozeduren, wie man eine Reihe von Handlungen ,kompetent hervorbringt; schließlich das, was man als ein motivational-emotionales Wissen bezeichnen kann, d.h. ein impliziter Sinn dafür 'was man eigentlich wills, 'worum es einem geht und was ,undenkbarı wäre. Durch die Zuordnung zu einzelnen, historisch und kulturell spezifischen Praxiskomplexen setzt die Praxistheorie diese Wissensformen dabei nicht als runiversal, sondern als historischspezifisch, als ein letztlich kontingentes llocal knowledger (Geertz) voraus." (Reckwitz 2003: 292)

Die Ergebnisse dieser Arbeit, die u.a. zeigt, dass die Fachpersonen kein explizites Verständnis von Jugendgewaltprävention äußern, lassen es fraglich erscheinen, inwiefern Prävention als Praktik überhaupt denkbar ist, insoweit der Begriff der Praktik überhaupt ernst genommen wird. ${ }^{6}$

6 | Auch auf programmatischer Ebene wird von "Praktiken" gesprochen im Sinne einer "Good Practice" oder "Best Practice". Diese Begriffe beziehen sich jedoch nur auf ein Ergebnis, das sich in der Statistik widerspiegelt. 



\section{Theoretische Diskussion der Ergebnisse}

\subsection{RESPONSIBILISIERUNG UND DIE INDIVIDUALISIERUNG VON RISIKO}

Eine am »sozialpolitischen Aktivierungsdiskurs « (Weyers 2006: 218) orientierte Soziale Arbeit, die ein »gutes « Leben bzw. eine »erfolgreiche Lebensgestaltung« als Ziel und Norm setzt und den Anpassungsprozess an die Gesellschaft letztlich in die Verantwortlichkeit der Jugendlichen selbst stellt, steht dem Ziel, Lern- und Bildungserfahrungen Jugendlicher zu rahmen und zu ermöglichen, im Sinne einer »möglichst offenen, wenig strukturierten, an den Interessen der Jugendlichen ansetzenden Arbeit« (Huber 2014: 42; vgl. auch Jordan/ Maykus/Stuckstätte 2015: 145ff.) gegenüber. Der schweizerische Diskurs über Jugendgewaltprävention ist dabei mit verschiedenen Diskurszusammenhängen verwoben, etwa mit Aktivierungs- und Risikodiskursen. Die »Verantwortungsübernahme wird zur Kondition und zur Legitimation von Leistungen - Responsibilisierung (oder Verantwortungsübertragung) wird zur Strategie, um moralisch verantwortliche Subjekte zu erzeugen« (Oelkers 2013: 164). Die Fachpersonen kommen als Adressatinnen und Adressaten kriminalpolitischer Programmatik zunächst selbst als diejenigen in den Blick, denen Verantwortung übertragen wird. Eine These ist, dass die Fachpersonen diese Verantwortung aufgrund der Ambivalenzen, die aus dem Bestreben, konkrete Aufträge zu erfüllen, und den eigenen Relevanzsetzungen - sowie den Bedingungen und Möglichkeiten, ihrer je spezifischen Handlungskontexte gerecht zu werden - hervorgehen, weiter an die Jugendlichen übertragen (responsibilisieren/ »überantworten«) und damit Risiken, die sich auf die Lebensphase Jugend im Allgemeinen beziehen, individualisieren. Damit wird eine Reproduktion der Objektivierung von Jugend als soziale Gruppe auf kriminalprogrammatischer Ebene in konkrete Handlungskontexte und damit auch Stigmatisierung und Ausschließung begünstigt. Die normative Erwartung an die Jugendlichen, sich der Gesellschaft anzupassen und dies auch noch »erfolgreich« oder in einer bestimmten Weise bzw. mit einer bestimmten Einsicht zu tun, bricht mit der gesellschaftskritischen Positionierung der Fachpersonen, aber auch mit ihrer grundsätzlich »Lebensweltorientierten Sozialen Arbeit« (Grunwald/ 
Thiersch 2004). Diese bezieht sich auf die »Ungleichheiten und Erosionen in der heutigen Lebenswelt« (ebd.: 15). Der Auftrag, ein »gutes« Leben zu führen, der im »Konzept der erfolgreichen Lebensgestaltung « seine Spitze findet, ist hingegen von der Zukunft her gedacht. Er gründet die Ziele Sozialer Arbeit auf hoch normativen Erwartungen in der Zukunft und ist somit ein hoch präventives Modell, insofern sich Prävention logisch auf eine bestimmte Zukunft bezieht und andere Szenarien dabei ausschließt sowie Verantwortung individualisiert (vgl. Cremer-Schäfer 2016; Kappeler 2016). Andererseits zeigt die hier vorliegende Arbeit, dass sich Jugendgewaltprävention auch an der Gegenwart orientiert, insofern sie Zukunft so verändern will, dass alles beim Alten bleibt. In der Orientierung am Präventionsdiskurs, der mit Diskursen über Risiko, Sicherheit und Aktivierung verschränkt ist, werden Risiken individualisiert, d.h. auf individuelle Jugendliche projiziert: Eine mit Normalisierung verknüpfte defizitorientierte Perspektive auf Jugend und die damit verbundenen normativen Vorstellungen von der Jugend in Abgrenzung zu den Erwachsenen werden dabei verallgemeinert. Das Konzept »Jugend «, das auch in der wissenschaftlichen Tradition sowie in medialen und politischen Darstellungsweisen im Sinne einer Naturalisierung von Defiziten, aus der Gewaltverhalten erklärt wird, negativ konnotiert ist (vgl. Anhorn 2002: 48f.; Bettinger 2007: 83), wird dabei insbesondere auf der Darstellungsebene der Erzählungen und Beschreibungen der Praktiken im Kontext offener Jugendarbeit individualisiert. So beschreiben auch Reder und Ziegler eine »deutliche Tendenz zur individualisierten Verantwortlichmachung der Betroffenen, eine Betonung von Frühinterventionen sowie die Bereitschaft, auf durchaus rigorose bis punitive Interventionsmuster zurückzugreifen, wenn die Betroffenen nicht die als notwendig erachtete Kooperationsbereitschaft zeigen.« (Reder/Ziegler 2011: 367) An dieser Stelle stellt sich nicht nur die Frage der Konsequenzen der Orientierung am Leitbild »Jugendgewaltprävention« für Soziale Arbeit, sondern auch für Jugendliche selbst. Die defizitorientierte Perspektive auf Jugendliche ist in den Darstellungsweisen der befragten Fachpersonen nicht nur ein Selbstverständnis, sondern wird auch in hohem Maße individualisiert, was Stigmatisierung, Ausschließung (vgl. Dollinger 2006: 146) und Skandalisierung (vgl. Cremer-Schäfer 2016: 18) begünstigt. Frau Blaum projiziert ihr Wissen über Gewalt auf einen Jugendlichen, indem sie ihn direkt damit konfrontiert. Relevant für sie ist weniger das aus dieser Situation resultierende »Verhalten« des Jugendlichen selbst, als vielmehr die Bestätigung, dass sich ihr Wissen als haltbar erwiesen hat. Insofern zielt auch ihre »Prävention« auf die Vermittlung »richtigen Wissens«, in diesem Fall bezüglich des Korans, ab. Auch Herr Tanner bewertet den Erfolg des Bewilligungsverfahrens nicht aus der Perspektive der Jugendlichen, sondern aus der Perspektive der Stadt, die mit dem Bewilligungsverfahren ihre Interessen durchsetzen kann. Die Individualisierung sozialstrukturell relevanter Risikomerkmale begünstigt nicht nur Stigmatisie- 
rung und Ausschließung, sondern ermöglicht auch die Rekonstruktion von Wissen (vgl. Cremer-Schäfer 1998: 132) und Macht, was als »Professionalisierung « über den Gebrauch kriminologischen Wissens angesehen werden kann. Stigmatisierung meint dabei, dass die Individualisierung von Risikomerkmalen, die ein Defizit ausdrücken und Gewaltverhalten erklären, andere individuelle situative kontextspezifische Merkmale und Problemlagen überdeckt. Somit geht mit der Adressierung Jugendlicher als eigenverantwortliche Subjekte hinsichtlich ihrer Anpassung an gesellschaftliche Strukturen gleichzeitig eine Dekonstruktion dieser in dem Sinne einher, dass sie mit ihren individuellen Merkmalen und Problemlagen nicht mehr als Adressatinnen und Adressaten Sozialer Arbeit anerkannt werden. »Konsequenterweise kennt diese präventive Logik kein Subjekt im Sinne der idealistischen Subjektphilosophie oder der kritischen Theorie, sondern nur ein >Subjekt<, das als Träger von Risikomerkmalen, als Risikosubjekt rekonstruiert wird.« (Lindenberg/Ziegler 2005: 618) Die Individualisierung von Risiken kann im Kontext von Jugendgewaltprävention für Fachpersonen einerseits als »Strategie« angesehen werden, ihre »Professionen« zu sichern und sich über den Gebrauch kriminologischen Wissens zu »professionalisieren«, indem sie sich damit an Problemdiskursen orientieren, die eben dieses Wissen zur Verfügung stellen. Andererseits sind die Handlungskontexte Sozialer Arbeit schwer vorstellbar ohne Bezug auf Subjekte (vgl. Castel 1983: 60), wie das abschließende Datenbeispiel veranschaulicht:

Interviewerin: "Und ja was sind des für Situation wie kann ich mir des vorstellen?" Frau Schock: "Ja 'Name einer Stadt kenn ich jetzt nicht ich weiß nicht welche dort die Brennpunkte sind aber ähm äh aber vielleicht im Quartier woo ganz viele Ausländer sind wo ganz viele (1.5) Kinder und Jugendliche auf der Straße sind und irgendwie rumschlendern ohne (.) wirklich etwas zu wissen oder (.) irgendwelche Leute anpöbeln und so weiter dass man dann so schräg (.) einfällt also wir haben keinen Standort wir haben keinen fixen Punkt (.) man kann uns nicht irgendwie telefonisch erreichen oder suchen oder äh anrufen es ist einfach (.) auf den Moment eine äh Intervention (.) die eben zuerstmal (.) erst nur ablenken möchte (.) die Achtung (.) auf dem was sie eigentlich anstellen wollte (.) kehrt."

Interviewerin: „Dann gehen Sie einfach los (.) Ihrer Gruppe und dann- ((lacht))“

B: "Ja (.) ja jetzt zum Beispiel am 'Name eines Platzes، in 'Name einer Stadt haben wir äh verschiedene ähm Interventionen gemacht und dort (.) ähm gibt's alles Mögliche es gibt Jugendliche es gibt die Mediengruppe es gibt die Ausländer die am dealen sind es gibt die Halbbetrunkenen die schon irgendwie auf der Suche (.) nach der Schlägerei sind und äh (.) da die (.) da die Aktivi also die Sportarten und die Musik sehr vielschichtig ist (.) ist die chance groß dass irgendjemand durch die Kampfkunst jetzt zum Beispiel (.) abgelenkt ist und interessiert (.) ist und dann so kann schon ein Gespräch entstehen und dann kommt man vielleicht wieder in drei Wochen und dann erkennt man sich wieder (.) also es ist sehr auf (.) es hat keine Struktur in dieser Hinsicht (.) es hat eine 
Haltung dahinter wie die 'Name des Projektesı auftreten müssen (.) sollen (.) was sie machen habe sie im Kopft aber es ist auch sehr viel (.) ähm Improvisation (.) und (.) der Situation angepasst (.) zu zu intervenieren und rechtzeitig zu stoppen es ist uns auch schon passiert (.) bevor es eskaliert weil es kann nicht sein dass wir mit unseren ,Name des Projektes، irgendwie der Grund (.) einer Schlägerei sind (.) und dann da wirklich den Moment (.) frühzeitig erkennen ok wir gehen."

I: "Hmhm (.) hmhm (2.5) spannend (.) und ähm (1.5) ja wie wie erkennt man grade so einen Moment?"

B: "Äh (.) des spüren Sie in der Stimmung es ist latent aggressiv es ist Alkohol im Spiel äh meistens äh es sind Gestik zwischen Gruppierungen es sind Blicke (.) e e e es ist vielleicht noch äh ein Polizist der äh streift es ist die Zeit in der Nacht (.) es ist die Art und Weise wie die Leute da herumlaufen (.) ähm in welcher Tonlage sie reden ((räuspert sich)) wieviel Raum sie einnehmen. (1.5) Ich kanns fast nicht beschreiben das spürt man einfach (.) oder ich spürs einfach." (Zeilen 197-218)

Frau Schock beschreibt das von ihr mitentwickelte Pilotprojekt, das sich dadurch auszeichnet, dass Jugendarbeiterinnen und Jugendarbeiter im öffentlichen Raum durch »Spontanität«, »schrägen« Bewegungen, Tanz oder Theater intervenieren. Die Interviewerin bittet um eine genauere Beschreibung des Geschehens, woraufhin Frau Schock das Verhältnis der Jugendarbeiterinnen und Jugendarbeiter zum öffentlichen Raum beschreibt. Sie hätten zum öffentlichen Raum eine bestimmte »Haltung«: Das, was sie machen, haben sie im Kopf und müssten trotzdem spontan und situationsgemäß (re-)agieren. Diese Interventionen laufen jedoch Gefahr, selbst Konflikte auszulösen. Das heißt, die Jugendarbeiterinnen und Jugendarbeiter müssten den richtigen Moment einschätzen können, um die Intervention zu stoppen. Für diesen Moment brauche es das richtige »Gespür«. Zentral an dieser Darstellung ist weniger der Versuch der Forscherin, den Sinn dieser Intervention zu verstehen, sondern die Beschreibungen des Ortes bzw. der Situation, in der interveniert wird, sowie die Beschreibung des Moments, an dem die Intervention besser gestoppt werden sollte, einer Reflexion zu unterziehen. Die Haltung der Projektmitarbeiterinnen und Projektmitarbeiter ist bereits geprägt von Erwartungen hinsichtlich der »Brennpunkte«, die sie aufsuchen. Frau Schock problematisiert dabei viele verschiedene Personengruppen und deren Handlungen, Einstellungen sowie deren antizipierte Handlungen, etwa »die vielen Ausländer«, »die Ausländer, die am dealen sind «, »die vielen Kinder und Jugendlichen«, die »auf der Straße sind irgendwie rumschlendern ohne (.) wirklich etwas zu wissen oder (.) irgendwelche Leute anpöbeln«, »Jugendliche«, »die Mediengruppe «, »die Halbbetrunkenen die schon irgendwie auf der Suche (.) nach der Schlägerei sind«»und so weiter« und »alles Mögliche«. Einerseits beschreibt Frau Schock ihre Haltung durch sehr genaue Kenntnis der Personengruppen, deren Handlungen und sogar geplanten Handlungen sowie deren Einstel- 
lungen - ein reichhaltiges Wissen über den Raum und die Situationen, das jedoch auch mit diffusen Elementen des Nicht-genau-Wissens verbunden ist. Der Moment der Intervention ist also durch Wissenselemente vorstrukturiert. Der Moment des »Interventionsstops « benötigt andererseits auch »Gespür für Stimmung und Aggressionsniveau«. Der Blick auf die Situation ist in hohem Maße geprägt von verschiedensten, bereits vorher problematisierten Merkmalen und vom Wissen über Ort und Situation, was in gewisser Weise für die Individualisierung und Generalisierung von Risiken spricht. Bröckling spricht hierbei von dem »präventiven Blick«, der

"den Verdacht generalisiert und Indizien aufzuspüren sucht, die auf künftige Übel hindeuten und an denen die vorbeugenden Maßnahmen ansetzen können. [...] Zum Risikosignal und Ausgangspunkt präventiven Handelns kann letztlich alles werden, was von Sollwerten abweicht oder sich als Vorzeichen solcher Abweichungen identifizieren lässt. Praktisch funktioniert Prävention als Ausrichtung und Selbstausrichtung an Normalitätsstandards, die damit den Status sozialer Normen erlangen." (Bröckling 2009: 211f.)

Insofern ist die Haltung, die Frau Schock beschreibt, hoch normativ und erfasst »alles Mögliche«, das zwar ihren Erwartungen bezüglich bestimmter Brennpunkte betrifft, aber nicht ihren Erwartungen hinsichtlich eines öffentlichen Platzes, wie er zu sein hat. Sie problematisiert nicht Individuen oder strukturelle Merkmale dieses Ortes, sondern bestimmte von vornherein als problematisch etikettierte Personengruppen, denen sie bestimmte problematische oder verdächtige Handlungsweisen unterstellt. Diese Personengruppen sind einem »generalisierten Verdacht« ausgesetzt, der als »Kehrseite einer solchen Institutionalisierung eines sozialen Problems und eine mögliche Folge von Skandalisierungen « (Krasmann 1997: 98; vgl. auch Schulz/Wambach 1983: 8) verstanden werden kann, was wiederum für eine sehr starke Orientierung Frau Schocks an Problemdiskursen spricht. Bezüglich ihrer Arbeit als Familientherapeutin grenzt sie sich hingegen sehr stark von durch Institutionen der Bildung und Erziehung zugeschriebene Problematisierungen ab. Auf die besondere Bedeutung von Raum und Prävention geht Schreiber wie folgt ein:

"Alle Orte, an denen sich ,falsches، menschliches Leben versammelt, werden dann zu Interventionspunkten. So löst die Prävention die Kriminalität von strukturellen und individuellen Bedingungen ab. Der Verweis auf den Ort hebt die Kriminalität an die Oberfläche und macht sie zu etwas Wirklichem. Der Prävention gelingt es hiermit, das komplexe Phänomen der Kriminalität in eine einfache räumliche Information zu übersetzen." (Schreiber 2011: 182)

So beobachten auch Michael Lindenberg und Holger Ziegler, dass die an Prävention orientierten Institutionen weniger $»$ Disziplinierung von Individu- 
en« als von »Personengruppen, kriminogene[n] Räume[n], Situationen und Gelegenheiten fokussierten Interventionen mit dem Ziel einer risikoarmen Gestaltung von Alltagsituationen « (Lindenberg/Ziegler 2005: 620f.) in den Mittelpunkt stellen. Bezüglich eines »präventiven Blicks« bzw. eines "generalisierten Verdachts « wird auch noch einmal auf Herrn Tanner verwiesen, der vermutet, die Jugendlichen, die in seine Einrichtung kämen, seien mit häuslicher Gewalt konfrontiert. Gleichzeitig gibt er jedoch an, dies eigentlich nicht zu wissen. Trotzdem sieht er die Familie als relevanten »Interventionsort« an, zu dem er jedoch leider keinen Zugang hat. Die hier vorliegende Arbeit weist darauf hin, dass Soziale Arbeit über die seit Jahrzehnten zunehmende Orientierung an Kriminalitätsdiskursen (vgl. Schreiber 2011: 16) nicht nur stigmatisierend und ausschließend auf Individuen einwirkt, sondern auch selbst einen Autonomieverlust erleidet (vgl. Anhorn/Bettinger 2002: 247; Dollinger 2015: 54; Groenemeyer 2015: 36). Folglich wird sozial Arbeit

»nicht nur Teil eines Ordnungsdiskurses, sie befördert damit auch die alltagstheoretische Vorstellung eines kategorialen, qualitativen Unterschieds zwischen einer Mehrheit von sozial Angepassten und ,Funktionstüchtigen und einer Minderheit von Problemgruppen und -individuen, von 'Auffälligen und 'Störern`, denen ein ganzes Bündel spezifischer psychosozialer Attribute und Persönlichkeitsmerkmale zugeschrieben wird. Aus Verschiedenheiten werden so grundsätzliche Unterschiede konstruiert und verfestigt. (Anhorn/Bettinger 2002: 250)

Gleichzeitig geht der Autonomieverlust aber mit der Möglichkeit einher, die eigene Expertise bzw. »Profession« nach außen hin zu legitimieren. Die Fachpersonen stellen implizit und explizit dar, dass sie sich aufgrund der von außen an sie herangetragenen Erwartungen dazu angehalten sehen, ihre vermeintlichen Erfolge sichtbar zu machen, was sie gleichzeitig problematisieren. Huber spricht in diesem Zusammenhang von Prozessen der

„Fremd- als auch der Selbstaktivierung bei den Akteuren der Jugendarbeit. Zum einen stehen die Träger im Fokus der Aktivierung von außen. Sie geraten verstärkt unter Rechtfertigungsdruck, denn innen wird mangelnde Effizienz und Effektivität unterstellt. [...] Veränderte Förderungs- und Finanzierungsbedingungen können so einen tiefgreifenden Wandel der grundlegenden Charakteristika der Jugendarbeit forcieren. Es geht dann nicht mehr um eine möglichst offene, wenig strukturierte, an den Interessen der Jugendlichen ansetzende Arbeit, sondern um die Implementierung von extern gesetzten und interessensunabhängigen curricularen Elementen und Programmen - nicht mehr um potentiell alle, sondern nur noch um die als problematisch identifizierten Jugendlichen und Jugendgruppen. Auch steht nicht mehr die Förderung von Selbstbestimmung, Bildungsprozessen, Freiräumen etc. oben auf der Agenda. Vielmehr findet sich dort die Forderung nach der Kontrolle und Anpassung der Jugendlichen [...]." (Huber 2014: 41f.) 
Die hier vorliegende Arbeit weist darauf hin, dass die Fachpersonen diese Prozesse teilweise sogar explizieren, kritische Stellungnahmen zumindest formulieren und versuchen, ihre Arbeit möglichst nah an den Bedingungen und Möglichkeiten der je spezifischen Handlungskontexte zu realisieren. In ihrer Orientierung am »Präventionsdiskurs« werden sie jedoch in ihren »Professionen « bedroht und gleichzeitig dazu bemächtigt, ihre »Professionen « zu sichern, indem sie beispielsweise auf vermeintliche Erfolge verweisen, Erfolge inszenieren oder Intervention nachträglich als Prävention (»Prävention für die Zukunft«) bezeichnen. Trotz des starken Spannungsverhältnisses appellieren sie an die Selbstverantwortlichkeit Jugendlicher, was angesichts ihrer Defizitperspektive auf diese paradox anmutet: Einerseits werden Jugendliche hinsichtlich kriminalitätsstatistisch als relevant gesetzter Merkmale als gefährlich bzw. gefährdet adressiert, andererseits werden sie hinsichtlich individueller oder situativer Risikoattributionen entadressiert und die Aufgabe, ihr Leben erfolgreich und glücklich zu gestalten, wird ihnen selbst überlassen (vgl. Schreiber 2011: 58f.). Damit besteht die Gefahr, dass Soziale Arbeit ihre Autonomie verliert und nur noch dazu dient, Wissen und Deutungen, die für ihren Kontext als relevant an sie herangetragen werden, zu reproduzieren. Im Kontext eines an Standardisierung von Projekten der Jugendgewaltprävention im Sinne einer flächendeckenden Implementierung sog. »evidenzbasierter« Projekte interessierten kriminalpolitischen Programms kann die Orientierung der Fachpersonen daran als eine Form von Subjektivierung angesehen werden. Soziale Arbeit, insbesondere die Jugendarbeit, droht damit zwar die Gefahr, einen Autonomieverlust ihres auf Beziehungsarbeit beruhenden Verständnisses von Prävention zu erleiden, »professionalisiert« sich aber gleichzeitig als Nutzerin einer gesellschaftlich anerkannten Wissensform, nämlich kriminologischen Wissens. Auf Basis dieser Ergebnisdiskussion wird auf zwei Problembereiche, die derzeit im Kontext Sozialer Arbeit diskutiert werden, fokussiert. Dies sind zum einen das Verhältnis von Kritik und Sozialer Arbeit und zum anderen das Verhältnis von Sozialer Arbeit und sozialer Kontrolle. Der letzte Problembereich schließt bereits an die hier konstatierte Gefahr eines Autonomieverlusts Sozialer Arbeit an und wird insbesondere im Kontext der Kritischen Kriminologie diskutiert, die Prävention im Rahmen kriminalpolitischer Entwicklungen kritisch in den Blick nimmt. Jedoch stehen beide Problembereiche in einem Zusammenhang, da Soziale Arbeit - verstanden als Ordnungspolitik, die primär Kontrollinstanz ist - nicht mehr als autonom und kritikfähig anzusehen ist (vgl. Anhorn/Bettinger 2002: 223; Schierz 2013: 42). Kriminalpräventive Strategien werden durch die Grundannahme infrage gestellt, nach der

"Kriminalität kein beobachtbares Verhalten einzelner Täter darstellt, deren Ursachen erforscht werden können und keine Eigenschaft bildet, die einem individuellen Verhal- 
ten inhärent ist. Kriminalität gilt als Produkt vielfältiger Zuschreibungsprozesse und ist damit eine gesellschaftliche Erscheinung, die nur als gesellschaftlicher Konstitutionsprozess rekonstruiert werden kann. Zentral für die kritische Kriminologie ist demnach der Prozess der Herstellung von Kriminalität." (Althoff 2002a: 47)

Diese Grundannahme, die im Etikettierungsansatz begründet ist, geht so weit, die Ursachen von Kriminalität letztlich im Strafrecht zu sehen (vgl. Sack 2002). In Distanz zu einer radikalen Lesart dieser Grundannahme, stellt die vorliegende Arbeit die These auf, dass »Wissen« bzw. Wissensproduktion kriminologischer Inhalte über Jugend bzw. Jugendliche und deren Handlungsweisen diese als soziale Gruppe festigt, die dann durch Zuschreibungen adressierbar gemacht wird. Dieses Wissen kann von der Sozialen Arbeit, deren Adressatin bzw. Adressat eigentlich nicht »die Jugend « ist, sondern Individuen sind (vgl. Anhorn 2002: 57; Lindenberg/Ziegler 2005: 614; Reder/Ziegler 2011: 369), pauschal vereinnahmt und individualisiert werden. So besteht die Gefahr, dass nicht nur »die Jugend « als soziale Gruppe Zuschreibungen und Ausschließungsprozessen unterliegt, sondern einzelne Jugendliche in einen Status des Diskreditierbar-Seins geraten. Nichtsdestotrotz ist eine Kritik der Fokussierung auf die Erforschung von Ursachen bzw. die Orientierung Sozialer Arbeit daran, nicht nur im Kontext von Jugendgewaltprävention, grundlegend angebracht. So werden Einschränkungen der Handlungspraxis Sozialer Arbeit im Zusammenhang mit Risikodiskursen auch in anderen Kontexten beobachtet. Sebastian Meyer beispielsweise beobachtet im Kontext des Kinderschutzes des Allgemeinen Sozialen Dienstes eine Steigerung der Risikoorientierung zulasten einer Lebensweltorientierung, was er u.a. auch auf Aspekte der Generierung von Sicherheit zurückführt. Er stellt fest, dass

"der heutige Kinderschutzdiskurs das Arbeitsklima und die Arbeitspraxis maßgeblich beeinflusst. [...] Alle drei Typen [Typisierung der Mitarbeiterinnen und Mitarbeiter des Allgemeinen Sozialen Dienstes (ASD)] beklagen die immer umfassenderen Kontrollmechanismen, die letzten Endes sowohl die KlientInnen als auch die ASD-Mitarbeiterinnen kontrollieren und einschränken. Diese Veränderungen werden sodann unter der Schirmherrschaft der Absicherung mehr oder weniger billigend in Kauf genommen." (Meyer 2016: 131)

Die hier vorliegende Arbeit zeigt, dass im Kontext von Jugendgewaltprävention das Wissen über Ursachen und Risiken individualisiert wird, indem es auf Individuen projiziert wird. Dabei geht der Einbezug situativer, kontextueller, sozialstruktureller und insgesamt lebenslagen- und lebensformspezifischer Aspekte der Individuen weitgehend verloren, was dem »klassischen« sozialpädagogischen Auftrag, insbesondere im Kontext der Jugendarbeit, widerspricht. Die Fokussierung auf Ursachen fördert den sog. "psychosozialen Blick« zu- 
lasten eines »ganzheitlichen Ansatzes« (vgl. Rapetti 2016). Eine ursachenbezogene Verengung der Forschung schränkt jedoch den Blick auch auf andere Bereiche ein:

"Die Resonanz, die der biowissenschaftliche Diskurs gegenwärtig erfährt, lässt sich jedoch dahingehend deuten, dass die medial, politisch und dementsprechend zunehmend auch individuell als zunehmende Bedrohung und Endzeit-Symptome wahrgenommenen globalen Phänomene mit den herkömmlichen Modellen und Konzepten der Sozialwissenschaften nicht mehr zufrieden stellend zu erklären und politisch zu bewältigen erscheinen. Sozialpsychologisch und gesellschaftspolitisch allzu attraktiv sind Konzepte, welche von zentralen Mechanismen und Interaktionsdynamiken der globalisierten und ökonomisierten Weltgesellschaft gleichsam ablenken: Vereinfachende kausale Konzepte des Rekurses auf 'Naturı, 'primäre Ursachen`, 'vorgegebenes Wesen`, welche es neuro-bio-wissenschaftlich raufzudecken gilt. Damit einher geht die folgerichtige und folgenreiche Kapitulation vor der Herausforderung, die angedeuteten Risiken und Bedrohungen durch gesellschaftliche Maßnahmen lösen zu können, die Regression auf symbolische Politik und Populismus." (Böllinger 2010: 15).

Eine Fokussierung auf die Erforschung von Ursachen wird der Komplexität des Sozialen nicht gerecht. So ist die These dieser Arbeit, dass Sozialpolitik und Soziale Arbeit und damit das ohnehin komplexe Spannungsfeld, das diese beiden Handlungsfelder auszeichnet, kriminalitätspolitisch überformt werden (vgl. Benz et al. 2013: 8). Eines der grundlegenden Spannungsverhältnisse bezeichnet der Begriff des doppelten Mandats, das auch das Spannungsverhältnis zwischen Hilfe und Kontrolle thematisiert (vgl. Huber 2014: 79f.). Die Schwierigkeit, die Interessen der Klientinnen und Klienten Sozialer Arbeit und sozialstaatliche Interessen miteinander zu vereinbaren, spiegelt sich v.a. in den Schilderungen im Kontext offener und aufsuchender Jugendarbeit wider (Intervention vs. Prävention; Vertrauensarbeit vs. »Präventionsauftrag«). Diese Arbeit zeigt, dass dieses grundlegende Spannungsverhältnis, das Soziale Arbeit charakterisiert, im Kontext von Jugendgewaltprävention virulent wird und tendenziell zulasten der Klientinnen und Klienten aufgelöst wird. Es ist davon auszugehen, dass kriminalpolitische Zielsetzungen durch diskursive Vermittlung in die soziale Alltagspraxis hineinwirken, soziale Realitäten formen und Machtverhältnisse festigen (vgl. Anhorn/Bettinger 2002: 238f., Höllmüller 2009: 75). Eine ernsthafte kritische - »autonome« - Auseinandersetzung Sozialer Arbeit mit Gesellschaft ist aus dieser Lage heraus kaum denkbar. Zudem besteht die Gefahr der Stigmatisierung und des Ausschlusses Jugendlicher, indem Risiken individualisiert werden (vgl. Anhorn/Bettinger 2002: 247f.; Groenemeyer 2012: 35, 2015: 22). Allerdings gewährleistet eine Orientierung an kriminalpolitischen Diskursen den Professionen Sozialer Arbeit eine vermeintliche Befriedigung eines Sicherungsbedürfnisses. Diese 
Prozesse können im Kontext von Jugendgewaltprävention aktivierungspolitisch gelesen werden, insofern

"sich die Aktivierung im Rahmen von Präventionsprogrammen findet. Prävention gilt in der Jugendarbeit inzwischen als das zentrale Paradigma schlechthin. Daran scheint auch die sehr kritische Auseinandersetzung mit der Präventionsorientierung der Praxis der Jugendarbeit durch die Sozial- und Erziehungswissenschaft wenig zu ändern." (Huber 2014: 42)

Jugendgewaltprävention erschöpft sich beinahe in der Forderung, Jugendliche sollen ein »gutes« und »erfolgreiches« Leben führen, die zu einer Anforderung für Jugendliche wird, da sie dafür letztlich zur Verantwortung gezogen werden (und nicht gesellschaftsstrukturelle Bedingungen). Dabei zeigt sich, dass die hier befragten Fachpersonen ihre eigenen normativen Erwartungen an eine »gute« Lebensgestaltung durchaus reflektieren, letztlich aber doch zum Ausgangspunkt von Prävention machen. Dabei liegt gerade in der Reflexion von Normativität eine Chance, Kritik zu üben (vgl. Sandermann 2013: 293f.). Eine konsequente Kritikfähigkeit benötigt womöglich den Einbezug der normativen Erwartungen und Normalitätsvorstellung der Adressatinnen und Adressaten und damit eine am Individuum ansetzende Soziale Arbeit.

\subsection{Verantwortung als Subjektivierungsform}

Die Literatur ist sich darüber nicht einig, ob Foucault »das Subjekt« als erkenntnistheoretische Figur zum Ausgangspunkt seiner Analysen macht (vgl. Gehring 2008: 21) oder es Gegenstand seiner Analysen ist und damit eben nicht als erkenntnistheoretischer oder normativer Ausgangspunkt dient (vgl. Schrage 2012: 76). Die hier vorliegende Arbeit zielt weniger auf diese Unterscheidung ab, sondern begreift »das Subjekt« als erkenntnistheoretische Figur, die sich als Gegenstand der Untersuchung darstellt, nicht aber empirische Kategorie sein kann. »Das Subjekt« kann zwar nicht als anthropologische Grundkonstante zum normativen Ausgangspunkt von Forschung gemacht (vgl. ebd.: 78), aber auch nicht empirisch in den Blick genommen werden:

"Menschen sind Subjekt inrer Akte und Urteile in der Weise, dass sie sich zum Subjekt machen oder zum Subjekt gemacht werden; sie sind nie einfach Subjekt, sondern sie werden nur je Subjekt. So versteht man den Titel 'Subjekt nicht mehr als Eigenschaft einer Substanz, sondern als einen praktischen Modus, in dem Menschen miteinander sind." (Müller 2013: 61) 
In diesem Sinne beschreibt Subjektivität ein Verhältnis des Miteinander-Seins in einem bestimmten historisch-spezifischen Kontext. Verantwortung, verstanden als Subjektivierung, beschreibt ein solches Verhältnis des Miteinander-Seins als Relation (vgl. Vogelmann 2014: 125):

"Mit ,Verantwortung، ist im Folgenden mehr und weniger als ein Begriff gemeint: mehr als ein Begriff, weil ,Verantwortung، etwas in Praktiken Aktives ist, das Macht ausübt, Erkenntnisse produziert und Einfluss auf die Subjektivität derer hat, die ,Verantwortung, gebrauchen oder von inrem Gebrauch betroffen sind. Weniger als ein Begriff ist 'Verantwortung', wenn 'Begriff، einen philosophisch wohldefinierten, geschichtslosen Sachverhalt meint." (Ebd.: 20)

Die hier vorliegende Arbeit zeigt auf, wie die Fachpersonen verschiedener Bereiche als verantwortliche Subjekte im Kontext von Jugendgewaltprävention angerufen werden und sich selbst im Verweisen auf kriminalpolitische Programmatik als solche darstellen. Der Modus dieser Verantwortung kann jedoch als hoch ambivalent beschrieben werden, da die bloße Zuschreibung von Verantwortung noch nichts darüber aussagt, wie diese Verantwortung zu gebrauchen ist. Die Fachpersonen werden aber nicht nur in ihrer Verantwortung angerufen, sondern auch in ihrer Verantwortlichkeit, d.h. bezüglich des Gebrauchs der Verantwortung. Sie sollen nicht nur Jugendgewaltprävention realisieren, sondern »gute« bzw. »erfolgsversprechende« Jugendgewaltprävention. Im Sprechen über ihre Aufträge und Erwartungen nehmen die Fachpersonen wiederum normative Adressierungen der Jugendlichen vor, indem sie ihre Erwartungen bezüglich eines guten Lebens - und wie dieses zu erreichen ist - in den Verantwortungs- und Verantwortlichkeitsbereich der Jugendlichen selbst stellen. In dieser Hinsicht kommen in der hier vorliegenden Arbeit drei Verantwortungsrelationen in den Blick: die Fachpersonen als diejenigen, denen Verantwortung zugeschrieben und diese auch tragen und als diejenigen, die Verantwortung zuschreiben, sowie die Jugendlichen, denen Verantwortung zugeschrieben wird. Fachpersonen sind als Subjekte anzusehen, insofern als »die zweite Position - von der aus die Verantwortungsrelation artikuliert wird - eine Subjektposition sein muss, weil gegenwärtig keiner Objektposition diskursive Fähigkeiten zugestanden werden « (ebd.: 126). So stellt sich eine bestimmte Weise der Verantwortung, wie Prävention als Ergebnis präsentiert werden soll, als nicht einlösbar heraus, weil sie mit anderen Weisen der Verantwortung, z.B. der Vertrauensbeziehung, in einer konflikthaften Beziehung steht. Für einzelne Fachpersonen können diese widersprüchlichen Erwartungen in bestimmten Situationen Unsicherheiten und Dilemmata erzeugen, derer sie sich wiederum über bestimmte Strategien bemächtigen können, z.B. indem sie Erfolge als »präventive Erfolge« ausweisen, obwohl sie diese aus dem eigenen Verständnis heraus eigentlich nicht als solche deuten. In gewisser 
Weise zeigt sich hier eine Widerständigkeit gegenüber Zuschreibungsprozessen. Für die Fachpersonen kann eine Orientierung an eigentlich nicht einlösbaren Verständnissen von Prävention jedoch auch als Bedeutungsgewinn angesehen werden, insoweit sie ihre »Professionen« darin legitimieren. Eben diese Differenz bzw. Ambivalenz kann als Subjektivierung verstanden werden, insofern als Subjektivierung »die in Institutionen stattfindende Verwandlung empirischer Einzelmenschen in solche, die sich als Subjekte begreifen und als Individuen handeln, bezeichnet (Schrage 2012: 80). In dem hier untersuchten Kontext können aber auch »die Jugendlichen« als Subjekte verstanden werden, die für die Ziele von Jugendgewaltprävention, ein »normales«, »angepasstes«, »gutes« und sogar »erfolgreiches« Leben zu führen, letztlich selbst verantwortlich gemacht werden. Dieser Logik nach kann nicht die Reduktion von Jugendgewalt Ziel von Jugendgewaltprävention sein, sondern die »Unveränderung« von Jugendgewalt. Ziel von Jugendgewaltprävention ist hier vielmehr das angepasst sein »der Erwachsenen«. In dieser Hinsicht geht mit der Normalisierung von Jugendgewalt eine Normalisierung »der Jugendlichen« im Sinne einer »Umwandlung von Menschen in Subjekte [...]« einher: »[...] dem Wortsinn nach geht es um die Anpassung von Verhalten an Normen und/ oder Normalität« (ebd.: 8o). Infolgedessen kann Jugendgewaltprävention als Subjektivierungstechnik verstanden werden, die nicht nur Jugendgewalt, sondern auch Jugendliche sowie die Fachbereiche Sozialer Arbeit »normalisiert« und somit anpasst.

"'Subjektivierung durch Normalisierung, bezeichnet daher, bringt man das hier nur knapp skizzierte Konzept auf einen allgemeinen Nenner, das In-Bezug-Setzen von Subjekten zu den nur in artifizieller Form manifestierbaren Massenprozessen in modernen Gesellschaften, mit dem Ziel, dauerhafte Weltverhältnisse zu etablieren, wie weitgehend selbsttätig Orientierung an der sich permanent wandelnden Wirklichkeit der modernen Gesellschaft gewährleisten." (Ebd.: 81)

Jugendgewaltprävention, verstanden als Subjektivierungstechnik, zielt, wie die vorliegende Arbeit aufzeigt, eben auf die Aufrechterhaltung der Generationenverhältnisse sowie der Apparate und »Professionen«, die sich zur Realisierung dieses Ziels bereits etabliert haben. Es kann angenommen werden, dass die Zuschreibung kollektiver Verantwortung nicht als Spezifikum des Diskurses über Jugendgewaltprävention, sondern als diskursanalytischer Gegenstand (Kategorie) zu betrachten ist, der insbesondere öffentliche Diskurse strukturiert. So beschreibt Reiner Keller, dass diese einen »typischen Hybridcharakter« aufweisen, insofern als

"kollektive Akteure aus unterschiedlichen Kontexten (z.B. aus Wissenschaft, Politik, Wirtschaft) bei der Auseinandersetzung um öffentliche Problemdefinitionen durch die 
Benutzung einer gemeinsamen Grunderzählung koalieren, in der spezifische Vorstellungen von kausaler und politischer Verantwortung, Problemdringlichkeit, Problemlösung, Opfern und Schuldigern formuliert werden " (Keller 2001: 133).

Somit fungiert die Zuschreibung kollektiver Verantwortung als Grunderzählung, die den öffentlichen und den Fachdiskurs inhaltlich strukturiert, insofern als »Bedeutungen in den Diskursen nicht als lose Zeichenpartikel vorliegen, sondern in strukturierten Formen, als typisierte und typisierbare Schemata. Diese werden in der diskursspezifischen Textproduktion, im Deuten und Handeln der in den Diskurs eingebundenen Akteure aktualisiert « (ebd.: 131). Die Beobachtung der Konstruktion kollektiver Verantwortung muss also nicht als Spezifik »des Präventionsdiskurses « verstanden werden, leitet aber weiter zur Frage, inwiefern die Fachpersonen als Subjekte eines spezifischen Diskurses anzusehen sind, inwiefern sie also in ihren Handlungsorientierungen daran anschließen. Über die Zuschreibung kollektiv-»prospektiver Verantwortung « (Adler 2009: 91) fokussiert der Diskurs auf die Notwendigkeit eines gemeinsamen, auf die Zukunft gerichteten Handelns, wie Verweise auf utopische und dystopische Szenarien verdeutlichen. Jedoch lässt sich prospektive Verantwortung nicht als »handlungs-, sondern ereignis- und zielorientiert« begreifen:

"Prospektive Verantwortung zu übernehmen, macht es zwar in vielen Fällen erforderlich, dass man aktiv Maßnahmen zum Erhalt eines Zustandes ergreift und es nicht einfach bei der Unterlassung von Handlungen belässt, aber dies gibt nicht vor, welche Maßnahmen es sind - eine Problematik, deren Schärfe zunimmt, je unklarer das Ziel formuliert wird." (Ebd.: 91)

Die Ziel- und Ergebnisorientierung kann dazu führen, dass die Fachpersonen Erwartungen ausgesetzt sind, die sie aber nicht in ihre Alltagspraxis, die bestimmten Zugzwängen, Restriktionen und Rahmenbedingungen unterliegt, integrieren können. Die Beobachtung der Konstruktion kollektiver Verantwortung und deren Responsibilisierung kann jedoch im Kontext von Kriminalität auch als Spezifik des Diskurses angesehen werden, insofern als Studien auf die »Verschränkung des transformierten diskursiven Operators Verantwortung mit der Transformation der Erfahrung Kriminalität in den Präventions- und Strafpraktiken« (Vogelmann 2014: 183) hinweisen. Demnach kann Responsibilisierung als Kriminalitätsdiskursen inhärente Strategie begriffen werden, die Erfahrungen von Kriminalität transformiert (vgl. ebd.: 199f.). Die hier vorliegende Arbeit geht davon aus, dass der Diskurs um Jugendgewaltprävention eingebettet ist in Diskurse über Risiken, Sicherheit und Aktivierung. Der Verweis auf (vermeintliche) Erfolge und deren Inszenierung können als Orientierung an einem solchen beschaffenen Diskurszusammenhang angesehen werden, mit dem Ziel einer Legitimation der Tätigkeit, infolge einer Zuschrei- 
bung von Verantwortung. Somit ergibt sich im Kontext von Risikodiskursen eine »andere Form der Responsibilisierung für Organisationen der Problemarbeit, die sich in ihrer Arbeit und im Nachweis ihrer Wirksamkeit erhöhten Legitimationsanforderungen ausgesetzt sehen und die aus diesem Grund auf Risikodiskurse und Risikotechnologien zurückgreifen « (Groenemeyer 2015: 26f.). So problematisiert die kriminalpolitische Programmatik nicht jugendliches Handeln, sondern die soziale Gruppe der Jugendlichen. Im Gegensatz zu Schreibers Beobachtungen zielt das in der vorliegenden Arbeit untersuchte Programm darauf ab, Verantwortung, auf die Zukunft gerichtet, auch kollektiv zu praktizieren und nicht im Sinne einer Lösung kontextspezifischer Herausforderungen zu individualisieren, sondern an die diskursspezifischen Problematisierungsweisen anzuschließen. Im Kontext werden also nicht individuelle Jugendliche, »Fälle«, in Bezug auf ihr Handeln problematisiert, sondern die Gruppe der Jugendlichen allgemein. Jugendgewaltprävention zielt dabei letztlich nicht auf die Reduktion von Jugendgewalt, sondern auf deren Aufrechterhaltung ab, die für die Reproduktion eines Präventionsapparates, der tief in der »friedlichen Gesellschaft« verankert ist, basal ist. Die Zuschreibung von Verantwortung und Verantwortlichkeit wird auch von der Forscherin aufgegriffen und individualisiert, was als eine Eigenart von »Experteninterviews « verstanden werden kann (vgl. Meuser/Nagel 2010: 377). Einerseits reproduziert sie damit die Grunderzählung bzw. Grundstruktur des Diskurses um Jugendgewaltprävention, andererseits ermöglicht sie den Fachpersonen, sich dazu zu positionieren, was im Rahmen der Konferenzen so kaum vorgesehen ist. Damit wird ermöglicht, sich den Subjektivierungsweisen der Fachpersonen anzunähern. Die vorliegende Arbeit beobachtet, dass das »Nationale Präventionsprogramm Jugend und Gewalt« nicht »Jugendgewalt«, sondern »die Jugendlichen« bzw. »die Jugend « problematisiert. Jugendgewalt fungiert dabei als Leerformel (vgl. Topitsch 1960), die es ermöglicht, ein sehr breites Spektrum an Fachpersonen in seiner kollektiven Verantwortung anzurufen. Auch Fritz Sack beschreibt aus der Perspektive eines gesellschaftshistorischen Kontexts, dass sich Prävention aus dem Monopol des Strafrechts heraus mehr und mehr in außerstaatliche Institutionen verlagert habe: »Die damit gesetzten restriktiven Möglichkeiten einer strafrechtlichen Kriminalprävention haben zunehmend zu einem über das Strafrecht hinausreichenden Präventionsverständnis geführt, das andere staatliche und öffentliche Akteure in präventive Verantwortung und Zuständigkeit gebracht hat.« (Sack 1995: 444) Folglich würden mehr und mehr Fachbereiche in ihrer Verantwortung für Gewaltprävention angerufen und könnten sich in dieser Hinsicht professionalisieren. Er beschreibt, dass sich die Geschichte Sozialer Arbeit eigentlich auch als Geschichte der Prävention lesen lasse. Dies sagt nichts über das »wie« der Prävention aus, jedoch lässt es das »Nationale Präventionsprogramm Jugend und Gewalt« in seinem Bestreben, all diese Fachbereiche und Professionen wieder 
zusammenzuführen und zu vereinheitlichen, in einem seltsamen Licht erscheinen. Inwiefern lässt sich Verantwortung nun als Subjektivierungsform weiter spezifizieren? Im Anschluss an Foucault steht Verantwortung in einem engen Zusammenhang mit (Handlungs-)Macht. Diese geht dabei immer mit einem Autonomieverlust einher:

"Während einige Theoretiker die Kritik der Souveränität als Zerstörung der Handlungsmacht missverstehen, setzt meiner Ansicht nach die Handlungsmacht gerade dort ein, wo die Souveränität schwindet. Wer handelt (d.h. gerade nicht das souveräne Subjekt), handelt genau in dem Maße, wie er oder sie als Handelnde und damit innerhalb eines sprachlichen Feldes konstituiert ist, das von Anbeginn an durch Beschränkungen, die zugleich Möglichkeiten eröffnen, eingegrenzt wird." (Butler 2006: 31f.)

Die Frage, die sich im Anschluss daran stellt, bezieht sich mit Blick auf die dieser Arbeit zugrunde liegende Forschungsfrage darauf, inwiefern die Fachpersonen in der Zuschreibung kollektiver Verantwortung auch in ihrem Handlungskontext handlungsmächtig werden? Die Beobachtung, dass die befragten Fachpersonen ein eigenes allgemeines Präventionsverständnis haben, das sich auf individuelles Handeln bezieht, d.h. sich an Fall und Situation orientiert und nicht auf soziale Gruppen im Allgemeinen zielt, spricht dafür, dass sie in ihrem Kontext nicht direkt und nicht nur an kriminalpolitische Programmatik anschließen. Jedoch werden sie in ihrem allgemeinen Präventionsverständnis von einem spezifischen Verständnis von Jugendgewaltprävention torpediert. Sie sind sich also bestimmten Erwartungen bewusst, wie sich an der von ihnen beschriebenen Notwendigkeit zeigt, Jugendgewaltprävention in irgendeiner Art und Weise zu messen oder als Erfolgsgeschichte zu inszenieren. So weist beispielsweise Herr Unterwasser seine Erfolge auf Basis von Berichten und medialer Inszenierung als Erfolge seiner Prävention aus, obwohl es sich dabei aus seiner Perspektive eigentlich nicht um Prävention handelt und er medialen Inszenierungen kritisch gegenübersteht. Die Positionierungen der Fachpersonen zu den von außen an sie herangetragenen explizit und implizit wahrgenommenen Aufträgen können als »Aneignung von Transformation « (Herrmann 2007: 300) angesehen werden und somit als Orientierung am Präventionsdiskurs. Diesem gegenüber distanzieren sie sich aber auch, deuten ihn um und bespielen ihn, was als eine Form von Widerständigkeit gelesen werden kann, insofern als die Fachpersonen sich dem Regiertwerden entziehen, indem sie die damit verbundenen Erwartungen nicht oder nicht gänzlich internalisieren und sich zu eigen machen (vgl. Bröckling/Krasmann 2010: $36 f$.). Cora Herrmann zeigt in ihrer Analyse der Positionierungen von Sozialarbeiterinnen und Sozialarbeitern zu Anforderungen an die Qualität ihrer Arbeit vier Figuren der Aneignung der Transformation "guter Arbeit« auf. Die erste dieser Figuren zeigt sich auch in dem hier vorliegenden analysierten Datenmaterial: 
"In allen Interviews taucht die Figur eines 'Außen، auf. Qualitätsentwicklung wird thematisiert als 'Prozess, der von außen (in die Einrichtungen) rein getragen worden ist، (I 5, S. 6). Die Entwicklung käme von außen und wirke nach Innen - - in die Einrichtungen. Zwei Orte erscheinen auf diese Weise. Damit wird der Debatte eine Herkunft jenseits der eigenen Verortung zugeschrieben. Die Wirkung der zwei Orte aufeinander wird als nicht reziprok und gleichberechtigt, sondern als einseitig und hierarchisch beschrieben: [...] Die serzwungene، Handlung ist an einen Adressaten im Außen gerichtet. Die 'Qualität der eigenen Arbeit darzustellen, erscheint als Vorgabe, als Regierung der anderen. Die Aktion wird im Außen verortet, die zu einer Reaktion im Innen führt. [...] Mit der Verortung im Außen werden unterschiedliche Formen des Handelns denkbar: Formen der Abgrenzung, des Widerstandes, der Relativierung oder die Variante, sich das Außen zu eigen zu machen. Welche Funktion übernimmt das Außen in den vorliegenden Aneignungsweisen? Es zeigt sich eine Relativierung des Einflusses von außen: [...] Hier wird Fremd- zur Selbstregierung, der Impuls jedoch bleibt im Außen verortet." (Herrmann 2007: 301)

Auch in den hier interpretierten Interviews stellen die befragten Fachpersonen die von außen an sie herangetragenen Erwartungen und Aufträge dar, kontrastieren diese mit den Zugzwängen ihrer Arbeit im »Innen« ihrer Organisation oder Einrichtung und unterziehen diese einer Überprüfung hinsichtlich der Realisierbarkeit. Die Bedeutung dieser Figur zeigt sich bereits in den Einstiegspassagen, in denen sich die Fachpersonen mit diesen Erwartungen und Aufträgen - implizit durch die Einstiegsfragen oder vielleicht gar bereits durch das Anschreiben - konfrontiert sehen und sich zunächst davon distanzieren. Die Beobachtung, dass sich die Fachpersonen der Nichterfüllbarkeit eines spezifischen Auftrags bezüglich der Jugendgewaltprävention durchaus bewusst sind, plausibilisiert Verantwortung als Subjektivierungsform, wie sie Frieder Vogelmann im Anschluss an Friedrich Nietzsche beschreibt. Sie unterscheidet sich eben durch Bewusst-Sein von dem Konzept Foucaults und (in dessen Anschluss) Butlers sowie darin, dass Verantwortung im Kontext einer moralisch-ethischen Perspektive gedacht wird. »Es ist sowohl das Bewusstsein der Zurichtung und damit ein raktives< Wissen um das eigene Unterworfen-Sein durch die >Sittlichkeit der Sitte< als auch das Bewusstsein der dank dieser Unterwerfung errungenen Macht und der Verpflichtung, die daraus erwächst.« (Vogelmann 2013: 153) Zudem macht die hier vorliegende Arbeit die Beobachtung, dass Fachpersonen Jugendgewaltprävention nicht als ihr primäres Anliegen ansehen und letztlich als individuellen Auftrag an die Jugendlichen reformulieren. So gesehen rufen die Fachpersonen die Jugendlichen in ihrer individuellen Verantwortung an, ein »gutes« und »gesundes« und »erfolgreiches « Leben zu führen, und übertragen die Verantwortung dabei letztlich auf die Jugendlichen. Jugendliche, die auf der Ebene kriminalpolitischer Programmatik als Objekte in den Blick kommen, werden in den Hand- 
lungskontexten der Fachpersonen zu Subjekten, indem an ihre individuelle Verantwortung appelliert wird. Letzten Endes profitieren die Fachpersonen von der Orientierung an kriminalpolitischer Programmatik, insofern als diese daran interessiert ist, ihre »Professionen zu sichern«, und sehen sich dazu in der Lage, ihre Arbeit als Erfolge auszuweisen, wobei sie ihren Handlungsspielraum durchaus als gefährdet ansehen. Andererseits kapitulieren sie vor dem Anspruch, tatsächlich spezifische Jugendgewaltpräventionsarbeit zu realisieren. Die Bedrohung des Kollektivs, insbesondere bezüglich seiner »Professionen«, ist den Fachpersonen also sehr wohl bewusst und sie schließen daran an, indem sie ihre Arbeit als Präventionsarbeit auszuweisen versuchen. Es bringt sie in eine Ambivalenz und in dieser sind sie nach Butler als Subjekte anzusehen, da gerade dort ihre »Souveränität schwindet«. Nach Butler sind sie aber auch als widerständige Subjekte anzusehen, da sie sich in ihren Praktiken den programmatischen Erwartungen entziehen und auch gesellschaftskritisches Bewusstsein zum Ausdruck bringen, das jedoch wiederum nicht praxisrelevant wird, da sie als Subjekte um ihre »Professionen« besorgt sind. Letztlich entziehen sich die Fachpersonen der Verantwortung für Jugendgewaltprävention, ohne ernsthaft ihre »Professionen« zu gefährden.

\subsection{THESEN}

Die hier als zentral herausgestellten Ergebnisse schließen an viele Beobachtungen an, die im Kontext von Prävention allgemein diskutiert werden. Die vorliegende Arbeit kontextualisiert diese Diskussionen in Bezug auf Jugendgewaltprävention in der Schweiz, wobei sie einige relevante Aspekte und Zusammenhänge fokussiert. Einige Einsichten und Zusammenhänge zeichnen diesen Kontext in spezieller Weise aus. Zunächst besteht eine der zentralen Beobachtungen dieser Arbeit darin, dass Jugendgewalt als diskursives Deutungsmuster in hohem Maße normalisiert wird und Prävention im Anschluss daran nicht primär auf die Vermeidung jugendlichen Gewaltverhaltens abzielt, sondern auf dessen Normalisierung. Jugendgewalt wird somit also als Deutungsmuster diskursiv reproduziert und als Gegenstand der Problembearbeitung etabliert und institutionalisiert. Jugendgewaltprävention zielt letztlich primär auf die Erhaltung eines etablierten Präventionsapparates. Die vorliegende Arbeit stellt außerdem heraus, dass im Rahmen des »Nationalen Präventionsprogramms Jugend und Gewalt« primär Fachbereiche Sozialer Arbeit als verantwortliche Akteure angerufen werden und diese die Verantwortung wiederum an Jugendliche übertragen. Diese Beobachtung verdeutlicht, dass Diskurse Praktiken im Kontext Sozialer Arbeit strukturieren und die Untersuchung dieser Transformationsprozesse relevant ist. Die Zuschreibung Sozialer Arbeit als verantwortliche Institution zur Bearbeitung sozialer Probleme 
(des sozialen Problems »Jugendgewalt«) steht dabei im Zusammenhang mit der Individualisierung von Risiken (Aktivierungspolitik), was als Konsequenz erhöhter Legitimitätsanforderungen gedeutet werden kann (vgl. Groenemeyer 2015: 40). Das zeigt sich bereits in den Interpretationen der Anfangssequenzen und kann nur dann angemessen reflektiert werden, wenn Interviews als Interaktionspraktiken in diskursiven Kontexten begriffen werden. Auf Basis dieser Ergebnisse werden fünf Thesen formuliert, die hier nicht abschließend diskutiert oder gar bestätigt, jedoch auf theoretischer Ebene weiterführend andiskutiert werden:

These 1: Jugendgewaltprävention ist als Technik anzusehen, die über Prozesse der Rekonstruktion von Jugendgewalt auf Bestandssicherung von »Professionen« Sozialer Arbeit, generationaler Ordnung und eines etablierten »Präventionsapparates« abzielt.

These 2: Die Fachpersonen befinden sich in einem hoch ambivalenten Spannungsverhältnis zu Jugendgewaltprävention als Technik. Einerseits profitieren sie vom Ziel der Bestandssicherung, die auch ihre Professionen betrifft. Andererseits sehen sie sich insbesondere in Bezug auf Ziele und Ergebnisse von Jugendgewaltprävention im Kern ihrer Arbeit bedroht und distanzieren sich von damit verbundenen Inhalten. Dieses Dilemma wird gelöst, indem sie die Verantwortung auf die Jugendlichen übertragen. So gesehen ist Jugendgewaltprävention auch eine aktivierungspolitische Technik.

These 3: Im Anschluss daran wird Soziale Arbeit kriminalitätspolitisch überformt und läuft Gefahr, ihre Autonomie und die dadurch bedingte Kritikfähigkeit zu verlieren.

These 4: Jugendgewaltprävention als Technik zielt darauf ab, dass sich Jugendliche gesellschaftlichen Strukturen anpassen. Gesellschaft wird dabei als Gesellschaft beschrieben, die nicht veränderbar ist bzw. nicht verändert werden soll. In den an Jugendliche vermittelten Zuschreibungen werden normative Erwartungen formuliert, wie ein »gutes«, »angepasstes« und »erfolgreiches« Leben zu führen ist.

These 5: Die Individualisierung kriminologischen Wissens durch Praktiken Sozialer Arbeit befördert nicht nur Ausschließungsprozesse und Stigmatisierungsprozesse von »Jugend « als soziale Gruppe, sondern auch von Individuen.

Fazit: Jugendgewaltprävention ist in dem hier beobachteten Kontext eine reaktionäre Kampfvokabel, die Unsicherheiten erzeugt, sich deren Bearbeitung zur Aufgabe macht und sich damit selbst reproduziert. Soziale Arbeit wird in 
der Konsequenz mit Unsicherheiten konfrontiert, die sie gar nicht minimieren kann und gar nicht minimieren soll. Der Gebrauch von Jugendgewaltprävention dient lediglich der Legitimation ihrer »Professionen«. Eine Reduktion von Unsicherheit wäre der Tod jeder Prävention. 



\section{Theoretische Anschlussdiskussion}

\subsection{JugendSOZIOLOGISCHE ANSChLÜSSE}

Die hier vorliegende Arbeit bestätigt die Sorge jugendsoziologischer Beobachtungen hinsichtlich der politischen und medialen Dauerthematisierung von Jugend als negativ von der Norm abweichende soziale Gruppe, die biologistisch, historistisch, kulturalistisch und naturalistisch begründet wird und wissenshistorisch zurückzuverfolgen ist. In diesen Prozessen wird Jugend als soziale Gruppe homogenisiert (vgl. Anhorn 2002: 50). In der Konsequenz werden Jugendliche unabhängig von ihren unterschiedlichen und jeweils vielseitigen Zugehörigkeiten sowie damit einhergehenden Herausforderungen vermehrt $\mathrm{zu} »$ Objekten von Disziplinierungsprogrammen« gemacht. Diese Prozesse »ermöglichen gerade keine gesellschaftliche Partizipation, sondern zielen auf Anpassung und Unterordnung ab wie sie zugleich auch Diskreditierung und Ausschließung zur Folge haben« (Stehr 2009: 108). Jugendgewaltprävention wird der Komplexität individueller Lebenszusammenhänge Jugendlicher nicht gerecht und richtet sich stattdessen primär auf die Bestandssicherung etablierter »Professionen« sowie auf die Aufrechterhaltung generationaler Machtverhältnisse. Diesen Zusammenhang konstatiert auch Anhorn, insbesondere bezüglich Jugend und Kriminalität, wobei die Kriminologie sich als Wissensform etabliert und sich Anerkennung sichert, während Jugendliche von Partizipation ausgeschlossen werden (vgl. Anhorn 2002: 54f.). Eine zentrale Legitimationsweise dieser Bestandssicherung, so zeigt die vorliegende Arbeit, erfolgt über die Normalisierung von Jugendgewalt, d.h. über die Rekonstruktion von Jugendgewalt als historisches, biologisches, kulturelles und naturgegebenes unveränderliches Deutungsmuster. Das damit verbundene Ziel, jugendliche Lebensläufe zu normieren, kann im Anschluss an Foucault auch als Disziplinierung begriffen werden, die Einschließung und Abweichung aneinanderkoppelt:

"Gesellschaftliche Institutionen wie die oben genannten [Militär, Fabrik, Schulen, Kliniken, Gefängnisse und Arbeitshäuser], aber auch Erziehungsanstalten oder Psychiatrien dienten nach Foucault dazu, durch Inklusion، Menschen an gesellschaftliche Regeln 
anzupassen und dadurch eine gesellschaftliche Normalität erst herzustellen. [...] In diesen ,Einschließungsmilieus` wurde Abweichung dabei immer und überall in den beobachtenden und bewerteten Blick genommen. Die 'Mikrophysik' der Macht - so Foucault - schreibt das normative Selbstverständnis durch die Überwachung und Anleitung in disziplinierenden Institutionen in die Körper der Individuen ein." (Kuhlmann 2012: 44; zit.n. Opitz 2007 und Foucault 1977)

Insofern ist Jugendgewaltprävention ein tradiertes und wenig innovatives Kontrollinstrumentarium, an das Jugendarbeit heute anschließt. Die befragten Fachpersonen, die Jugendgewaltprävention nicht in ihr allgemeines, ganzheitlich orientiertes Konzept von »Sozialer Prävention« integrieren können, haben dennoch einen Nutzen von der Orientierung an Jugendgewaltprävention und der diese steuernden kriminalpolitischen Programmatik, insofern sie ihre »Professionen« dadurch sichern, womit Stigmatisierungs- und Ausschließungsprozesse Jugendlicher jedoch weiter begünstigt werden. Vor diesem Hintergrund schließt die hier vorliegende Arbeit an kritische Auseinandersetzungen mit aktuellen jugendsoziologischen Fragestellungen an, insbesondere im Kontext »sozialer Probleme«. Eine der zentralen Fragen betrifft dabei das Konzept »Jugend «. Wie über Jugend forschen, wenn Jugend ein historisch hervorgebrachtes und dabei in vielseitige Problematisierungsprozesse eingelassenes Konstrukt darstellt, ein

"(implizit) normatives Konstrukt, das mit der Konstruktion der Jugend als dem Anderen, als Defizit, als Gefährdung und Gefährlichkeit in dem Maße der Entmachtung und Ausgrenzung von Jugendlichen dient, wie es damit gleichzeitig die Voraussetzungen und Legitimationen für eine (sozial- und kriminal-)politische und sozialpädagogische Intensivierung und Erweiterung der Kontrolle und Disziplinierung von Jugendlichen schafft" (Anhorn 2002: 48).

Laut Johannes Stehr ist »)Jugend< ebenso wie >Gewalt< kein beschreibender oder analytischer Begriff [...], er verweist stattdessen auf die über die Zuschreibung der Unreife unterstellte Notwendigkeit und Praxis der Erziehung« (Stehr 2009: 107) und appelliert an Partizipation und Subjektorientierung in Bezug auf Jugendliche. Seinem Verständnis nach macht gerade der Partizipationsbegriff auf die Ungleichverteilung von Beteiligung in Bezug auf unterschiedliche Personengruppen aufmerksam. Er schließt daraus, dass

"mit einem relationalen Verständnis sozialer Ausschließung zwangsläufig Fragen gesamtgesellschaftlicher, konflikthafter Macht- und Herrschaftsverhältnisse und die Veränderung gesellschaftlicher Institutionen und Strukturen, und nicht die Veränderung der Ausgeschlossenen, ihres Verhaltens, ihrer Einstellungen, ihrer Fähigkeiten etc. in den Vordergrund treten“ (Anhorn 2008: 37). 
Damit zeigen sich große Parallelen zur aktuellen Kindheitsforschung. In ihrer Untersuchung politischer Adressierungsweisen von Kindern stellt Doris Bühler-Niederberger fest, dass Kindheit als Objekt von Problemdiskursen, die eine »Gesamtgesellschaft« betreffen, konstruiert wird und Kinder dabei nicht als Personen mit individuellen Bedürfnissen und Problemlagen in den Blick genommen und angesprochen werden $(2007,2010)$. Politische Debatten ließen sich als Diskurse beschreiben, die Kinder als soziale Gruppe in ihrer Andersartigkeit und Hilfsbedürftigkeit von einer »Mehrheitsgesellschaft« ausschließen. Dem hält Bühler-Niederberger das Bestreben der Kritik einer »neueren und neuen Kindheitssoziologie« an einer verengten Perspektive »klassischer« Soziologen auf Kindheit entgegen, die »alle Fragen nach der Gegenwart, nach den aktuellen Erfahrungen des Kindes, nach seinem sozialen Handeln und seiner eigenen Sicht der Dinge ausblenden würden« (Bühler-Niederberger 2011: 168). Der Einbezug subjektiver Perspektiven von Kindern und Jugendlichen (vgl. auch Breidenstein/Kelle 1998; Kelle 1996) verunmöglicht auch den Begriff der Jugend, der »eine semantische Vereinheitlichung vornimmt, die der Realität nie gerecht werden kann« (Dollinger/Schmidt-Semisch 2011: 13). Im Anschluss daran würde eine kritische Jugendforschung bedenken, dass Jugend einerseits als gesellschaftlich hergestelltes Konstrukt zu beforschen ist und Jugendliche andererseits als soziale Akteure ernst zu nehmen sind, die ihre eigene Lebensrealität mitgestalten. So kritisiert die hier vorliegende Arbeit, dass sich Jugendgewaltprävention auf den Bezug wissenschaftlich generierten Wissens fokussiert, v.a. auf die Relevanz von Ursachen und Risiken hinsichtlich Jugendgewalt. Die kriminalpolitische Relevanz dieses Wissens beeinflusst das Wahrnehmen und Handeln professioneller Fachpersonen, womit Wissen und Macht institutionalisiert werden. In der Konsequenz werden Jugendliche verstärkt mit institutionellen und normativen Erwartungen konfrontiert und damit in ihren Handlungs-, Gestaltungs- und Partizipationsmöglichkeiten eingeschränkt. Das beste Beispiel aus den vorliegenden Daten ist Herrn Tanners Bewertung des Bewilligungsverfahrens. Herr Tanner, der eigentlich ein kritisches Verhältnis zur Politisierung von Jugendgewalt hat und die Entwicklungsmöglichkeiten Jugendlicher offen und ganzheitlich fördern will, spricht in seiner Reflexion dieses von der Stadt durchgesetzten Verfahrens Jugendlichen keinerlei Autonomie zu. Würden die Institutionen Sozialer Arbeit, so die Überlegung der hier vorliegenden Arbeit, selbst weniger in ihrer Autonomie begrenzt, hätten sie eher die Möglichkeit, Jugendliche als autonome Akteure ernst zu nehmen und müssten nicht mit ihren eigenen Konzepten und Leitbildern brechen. Es lässt sich, wie bereits erwähnt, beobachten, dass wissenschaftlich, politisch und medial aufgegriffene Konzepte von Jugend sich durch eine defizitorientierte Perspektive auf Jugend auszeichnen und durch diese legitimiert werden (vgl. Anhorn 2002: 48f.; Bettinger 2007: 83). Dies lässt sich (wissens-)historisch zurückverfolgen: Die Entstehung der Ideen von Kindheit 
und Jugend geht mit der v.a. durch die Aufklärung verfestigten Konzeptualisierung einer Trennung von Natur und Kultur einher (vgl. Postman 1982: 566 .). Kindheit und Jugend werden demnach dem Bereich der Natur zugeschrieben, den sie durch Erziehung überwinden können und sollen (vgl. ebd.). Dieses Konzept prägt die soziologischen Theorien zu Kindheit (vgl. Bühler-Niederberger 2011), insbesondere auch die »klassischen« jugendsoziologischen Theorien (vgl. Eisenstadt 1966; Tenbruck 1965) sowie die »klassischen« entwicklungspsychologischen Theorien (vgl. Erikson 1970). Diese Konzepte, die an Talcott Parsons' Strukturfunktionalismus anschließen, sehen die Anpassung von Individuen an Gesellschaft vor, insbesondere über Rollenübernahme. Sozialisation meint damit Anpassung an stabile, vorgegebene, nicht veränderbare gesellschaftliche Strukturen und damit deren Aufrechterhaltung (vgl. BühlerNiederberger 2011: 115). Individuelle Biografien werden dabei als »Normalbiographien « konzeptualisiert. Dabei gilt die Lebensphase »Jugend « als besonders virulent, da vorgesehen ist, dass in dieser die Rollenmodelle erprobt werden und eine stabile Ich-Identität entwickelt wird. Diese defizitorientierte Sichtweise auf Jugend ist anhand der vorliegenden Interviews rekonstruierbar, insbesondere am Beispiel der Geschichte Frau Blaums. In der Konsequenz wird auch dort Jugendgewalt normalisiert, werden die den Diskurs prägenden Risikofaktoren individualisiert. Dabei veranschaulicht die Frage nach der Legitimation von Prävention, dass die Fachpersonen in ihren eigenen Verständnissen von Prävention mit einer auf Wirksamkeit, Evidenz und Nachhaltigkeit abzielenden Deutung von Jugendgewaltprävention konfrontiert sind, die sie nicht in ihren professionellen Handlungskontext integrieren können.

\subsection{Gewalttheoretische Anschlüsse}

Ähnlich wie bei der Beforschung von »Jugend« geht es hier um die Frage, wie aus soziologischer Perspektive Gewalt untersucht werden kann, wenn Gewalt so stark problematisiert ist und kriminologischer Ursachenforschung eine derart prominente Rolle zugesprochen wird. Soll Gewalt als analytisches Konzept fungieren oder als Gegenstand in den Blick kommen? Ist beides denkbar? In der hier vorliegenden Arbeit kommt auch Gewalt als diskursiv erzeugter Gegenstand in den Blick und ist somit wie »Jugend «als Gegenstand »der Analyse nicht einfach gegeben«, sondern

"konstruiert, situiert und diskursiv erzeugt. Gewalt nun ist vor allen Dingen durch ihre moralisch-ethisch-politische Situierung gekennzeichnet. Man kann entweder innerhalb dieses Kontexts argumentieren, inn als gegeben voraussetzen, oder versuchen, inn sichtbar zu machen, man kann aber auch einfach so tun, als wäre er nicht vorhanden." (Kreissl 1997: 184) 
Diese Sichtweise bzw. Einsicht relativiert die kriminologische Vormachtstellung bezüglich der (Ursachen-)Forschung über Gewalt. Als ein großes Defizit kriminologischer Erforschung von Gewalt wird deren fehlende Definitionsarbeit wahrgenommen. Gewalt kommt dabei fast ausschließlich als (angezeigtes) delinquentes »Verhalten « in den Blick, das die unterschiedlichsten Ausprägungen aufweisen kann. ${ }^{1}$ Aber eine Aufzählung nicht anerkannten, unter Umständen strafbaren »Verhaltens«, das bereits durch historische Problematisierungsprozesse etikettiert ist, stellt noch keine Definition dar. Eines der Grundprobleme, das die Definition von Gewalt betrifft, ist deren Abgrenzung vom Machtbegriff (eine Übersicht dazu bietet Han 2011). Dies kann insbesondere auf die starke Rezeption der Theorie Galtungs zur strukturellen Gewalt Gewalt (vgl. Galtung 1975) zurückgeführt werden. Galtung unterscheidet primär zwischen »personaler« und »struktureller Gewalt«, wobei sich der Begriff der »strukturellen Gewalt« dem Machtbegriff Foucaults annähert:

"Andererseits nimmt es nicht Wunder, dass die personale Gewalt stärker im Blickpunkt der Aufmerksamkeit steht als die strukturelle Gewalt. Personale Gewalt zeigt sich. Das Objekt der personalen Gewalt nimmt die Gewalt normalerweise wahr und kann sich dagegen wehren - das Objekt der strukturellen Gewalt kann dazu überredet werden, überhaupt nichts wahrzunehmen." (Ebd.: 16)

»Personale Gewalt« hingegen, davon geht Galtung zumindest aus, ist sichtbar und somit anschlussfähig für weitere Handlungsoptionen. Die hier vorliegende Arbeit beobachtet, dass sich >personale Gewalt< nicht zeigen muss, sondern inszeniert werden muss, um zu einem politisch und sozialpädagogisch bearbeitbaren Gegenstand zu werden. Das Interesse der Prävention besteht in der Inszenierung und Rekonstruktion dieser Gewaltform. Galtung versucht, einen logischen sowie empirischen Wirkungszusammenhang zwischen den beiden Formen von Gewalt herzustellen - ein Versuch, der ohne befriedigendes Ergebnis bleibt:

"Die beiden Typen von Gewalt sind anscheinend empirisch nicht fester miteinander verknüpft als logisch - und was das letztere angeht, so besteht die ganze Übung in dem Versuch zu zeigen, dass sie als logisch voneinander unabhängig begriffen werden können, obwohl sie miteinander zusammenhängen: sie gehen ineinander über.u (Ebd.: 30)

Diese Typisierung erscheint künstlich, was die Bedeutung sozialkonstruktivistischer Zugänge zu Gewalt hervorhebt. Auch Pierre Bourdieu spricht von »symbolischer Gewalt« als moderne Herrschaftsform, die nicht als Zwang

1 Im Kontext von Jugendgewalt betrifft es die Verwendung von Kraftausdrücken über das Kicken von Coladosen bis hin zur Vergewaltigung. 
wirkt, sondern ganz ähnlich wie Foucaults Machtkonzept einen Willen, sich zu unterwerfen, einschließt:

"Es ist klar, daß man diese besondere Form von Herrschaft nur unter der Bedingung adäquat zu erfassen vermag, daß die naive Alternative von Nötigung und Einwilligung, von Zwang und Zustimmung überwunden wird. Symbolische Gewalt übt einen Zwang aus, der durch eine abgepreßte Anerkennung vermittelt ist, die der Beherrschte dem Herrschenden zu zollen nicht umhinkann. [...] Alle Macht hat eine symbolische Dimension: sie muß von den Beherrschten eine Form von Zustimmung erhalten, die nicht auf der freiwilligen Entscheidung eines aufgeklärten Bewußtseins beruht, sondern auf der unmittelbaren und vorreflexiven Unterwerfung der sozialisierten Körper." (Bourdieu 1997: 164f.)

Diese beiden prominenten Vertreter einer soziologischen Thematisierung von Gewalt haben einen großen Einfluss auf den Gebrauch eines über die Wissenschaft hinausgehenden weiten Begriffs von Gewalt. Dies kann gute Gründe haben, z.B. die im Kontext der Friedens- und Antidiskriminierungsbewegung stehende Sensibilität für Gewalt gegen Minderheiten, mit der das Konzept der Nullgewalttoleranz einhergeht, das in der offenen Jugendarbeit oder an Schulen in Form von Streitschlichterprogrammen Eingang findet. Für die Erforschung der Entstehung von Gewalt sind »weite« Gewaltkonzepte jedoch kontraproduktiv, da sie durch fehlende Abgrenzung von Machtprozessen und Strukturen sozialer Ungleichheit mit diesen verschwimmen und »tatsächliche« Gewalt gewissermaßen entschärfen. Die Subsummierung jeglicher - auf breiter gesellschaftlicher Basis Anstoß hervorrufender, da nicht einem »normalen« oder durchschnittlichen Modell entsprechender - Handlungsweisen unter den Gewaltbegriff verhält sich konform zu einer »wissenschaftlich« etablierten weiten Fassung des Gewaltbegriffs, der einer gewissen Pragmatik nicht entbehrt:

"Nun hängt alles davon ab, wie man 'Gewalt definiert. Dies ist eine überaus undankbare Aufgabe und die Vorschläge werden viele Leser kaum befriedigen können. Es ist indes nicht so wichtig, so etwas wie die Definition oder die Typologie zu finden, denn offensichtlich gibt es viele Formen von Gewalt. Vielmehr kommt es darauf an, theoretisch signifikante Dimensionen von Gewalt aufzuzeigen, die das Denken, die Forschung und möglicherweise auch das Handeln auf die wichtigsten Probleme hinlenken. Wenn das Handeln für den Frieden einen so wesentlichen Stellenwert einnimmt, weil es ein Handeln gegen die Gewalt ist, dann muss der Begriff von Gewalt so umfassend sein, dass er die wichtigsten Varianten einschließt, gleichzeitig aber so spezifisch, dass er die Basis für konkretes Handeln abgeben kann." (Galtung 1975: 8)

Die Entscheidung für den Gebrauch eines weiten Gewaltbegriffs ist eine ideologische: »Eine positive und erweiterte Bestimmung von Gewaltfreiheit soll 
dazu dienen, den Prozess der tendenziellen Universalisierung des Gewalttabus, die mit der gesellschaftlichen Verbreitung der Ächtung von physischer Gewalt einsetzte, voranzutreiben.« (Felten 2000: 34) Gebrauch und Verbreitung eines weit gefassten Gewaltbegriffs und die damit verbundene Omnipräsenz von Gewalt, was als eine diskursive Konstruktion von Gewalt zu verstehen ist, setzen jedoch die Gefahr frei, »tatsächliche « Gewalt zu nivellieren. ${ }^{2}$ Gewalt wird gewissermaßen durch Gewalt »bekämpft«, indem Gewalt diskursiv hergestellt wird. Paradebeispiel ist die Situation der Prävention, in der Gewalt, die nicht da ist, »bekämpft« werden soll. Hans Kritik an René Girards Interpretation von Opferritualen in präkolumbianischen Kulturen etwa zielt darauf, die Opfergaben nicht als Werkzeuge der Beschwichtigung der Götter zu lesen, sondern ihren Sinn in sich selbst zu sehen, als Manifestation von Gewalt, die ein Gegengewicht zu der Gewalt der Götter bzw. der Natur schaffen soll. Opferrituale sind nach Han nicht als Präventionsmaßnahmen zu interpretieren, sondern als repressive Mittel, um Gewalt zu »bekämpfen«:

"Die unzähligen Totenschädel, die den aztekischen Tempel schmücken, sprechen nicht die Sprache einer Gewaltprävention, sondern die einer aktiven Gewaltproduktion. [...] Man schützt sich vor Gewalt, indem man selbst aktiv Gewalt ausübt. [...] Die Ausübung von Gewalt steigert das Machtgefühl. Mehr Gewalt bedeutet mehr Macht. In der archaischen Kultur stellt die Macht noch kein Herrschaftsverhältnis dar." (Han 2011: 22f.)

Die Inszenierung von Gewalt und die Konstruktion von Bedrohungsszenarien kann in diesem Sinne als »aktive Gewaltproduktion« angesehen werden. Die historische Tabuisierung von Gewalt, die als Folge der Ablösung des Naturrechts durch das positive Recht einzuordnen ist (vgl. Benjamin 2006, 34f.),

2 | "Wird die Gewalt zum Chiffre für die allgemeine gesellschaftliche Negativität entgrenzt, so verschwimmt inr Begriffsprofil ganz. Galtungs Gewaltbegriff erfasst vor allem die Differenz von Macht und Gewalt nicht. [...] Die strukturelle Gewalt ist keine Gewalt im strengen Sinne. Sie ist vielmehr eine Herrschaftstechnik." (Han 2011: 101f.) Auch Michael Staudigls phänomenologische Kritik des in der soziologischen Theorie weit verbreiteten kriminologischen Konzepts von Gewalt trifft dessen Beschränkung auf sichtbare Gewalt, die messbar und quantifizierbar ist, vor dem Hintergrund der Ignoranz nicht sichtbarer Formen von Gewalt: "Yet, conversely even though these so-called ,renovators seek to analyze the dynamics of violent encounters and to lay bare their potential excessiveness, they also adhere to another kind of one-sided perspective. Driven by the idea of providing detailed descriptions of violence as it is experienced subjectively they focus on phenomena of visible violence and its seizable effects on real - i.e., embodied subjects. In doing so, they nevertheless neglect the various forms of invisible violence [...] these positions tend to restrict violence to that which is physical." (Staudig| 2007: 236) 
und die damit verbundene Verdrängung von Gewalt aus öffentlichen Räumen sind kein Zeichen dafür, dass Gewalt mehr und mehr aus der Gesellschaft verschwunden ist. ${ }^{3}$ "In der Moderne wird der brachialen Gewalt nicht nur auf der politischen Bühne, sondern auch auf fast allen gesellschaftlichen Ebenen zunehmend Legitimation entzogen.« (Han 2011: 14) Die hier vorliegende Arbeit definiert Gewalt zwar nicht, lenkt jedoch aus der Beobachtung von Gewalt als diskursiv erzeugtes Konstrukt den Blick auf Prozesse der Verfestigung von Machtverhältnissen über die Problematisierung von Gewalt aus unterschiedlichen Perspektiven. Insofern trifft die Beobachtung, dass den griechischen Göttern martialische Formen von Gewalt als ein öffentlich legitimiertes Mittel zum Zweck dienten sowie auch die Spiele im antiken Rom schlicht der Demonstration von Macht (vgl. Han 2010: 11f.), in gewisser Weise auch auf heute zu:

"Die Gewalt ist in der Vormoderne allgegenwärtig und vor allem alltäglich und sichtbar. Sie ist ein wesentlicher Bestandteil der gesellschaftlichen Praxis und Kommunikation. Darum wird sie nicht nur ausgeübt, sondern auch eigens zur Schau gestellt. [...] Die Gewalt und deren theatrale Inszenierung gehören hier wesentlich zur Ausübung von Macht und Herrschaft." (Han 2011: 12)

Macht und Gewalt treiben zwar im Laufe der Geschichte auseinander und stehen heute, wie hier gezeigt worden ist, in keinem strukturierten Verhältnis zueinander. Jedoch hat die inszenierte Gewalt, sei es in Medien, sei es auf der (politischen) Bühne oder im Kontext sozialpädagogischer Maßnahmen, einen zentralen Stellenwert für die Demonstration von Macht, Deutungsmacht und Wissen. Auch aufgrund dieser Beobachtung ist es zentral für die soziologische Erforschung von Gewalt, diese von Macht abzugrenzen - Analyseperspektive und Untersuchungsgegenstand damit zu trennen. Foucault hingegen grenzt Macht von Gewalt durch ein positives Verständnis von Macht ab. Freiheit ist dabei nicht als Gegenpol zu Macht, sondern dieser als inhärent anzusehen. Gewalt bezieht sich sodann auf Bereiche, in denen keine Freiheit mehr denkbar ist, und übt eine nur einseitige Wirkung auf Individuen aus. Gewalt benötigt, im Gegensatz zu Macht, das Subjekt nicht:

3 | "Die zunehmende Positivierung der Gesellschaft lässt auch jede Gewaltform, sowohl die physische als auch die psychische, als verwerflich erscheinen. Sie bedeutet jedoch nicht das Ende der Gewalt, denn Gewalt geht nicht nur von der Negativität des Anderen, sondern auch von dem Übermaß an Positivität aus. Die Gewalt der Positivität ist nicht privativ, sondern saturativ, nicht exekutiv, sondern exhaustiv. Sie beruht nicht auf der Exklusion, sondern auf der Exurbanz. Sie äußert sich nicht als Repression, sondern als Depression." (Han 2011: 96) 
"Die Gewalt zielt auf die Körper, auf Gegenstände oder bestimmte Entitäten, deren Form sie zerstört oder verändert, während ihre Kraft kein anderes Objekt besitzt als andere Kräfte, kein anderes Sein hat als das eines Verhältnisses: sie ist eine 'Handlung [action], die auf andere Handlungen, auf mögliche oder auf wirkliche, künftige oder gegenwärtige Handlungen، einwirkt, reine Gesamtheit von Handlungen, die auf potentielle Handlungen einwirkt. [...] Wenn die Macht nicht einfach Gewalt ist, so nicht allein deshalb, weil sie selbst über Kategorien läuft, die die Beziehung von Kraft zu Kraft ausdrücken (anregen, verleiten, einen Nutzeffekt erzielen usw.), sondern auch, weil sie im Verhältnis zum Wissen Wahrheit erzeugt, insofern sie zum Sehen und zum Sprechen bringt. Sie erzeugt Wahres als Problem." (Deleuze 1992: 99, 116)

Nicht nur Prävention ist eine Leerformel, die beliebig aufgefüllt werden kann (vgl. Lindner/Freund 2001; Reder/Ziegler 2011), sondern auch Gewalt als diskursives Konstrukt ist eine Leerformel, über die sich Gesellschaft reproduziert. Auf der Ebene der Praktiken wird zwar jugendliches Gewalthandeln problematisiert, aber in einer weiten Begrifflichkeit und nicht im Anschluss an Definitionen. So zielt eine dauerhafte politische Problematisierung von Jugendgewalt auf eine Sicherung der »Professionen« und der generationalen Machtverhältnisse. Inwiefern Jugendgewaltprävention wirksam ist, soll in diesem Zusammenhang gar nicht abschließend beantwortet werden und fällt in den Verantwortungsbereich der Jugendlichen selbst. Jugendgewaltprävention als soziale Praktik ist aus den Daten der hier vorliegenden Arbeit nicht rekonstruierbar, insofern sich die befragten Fachpersonen in der Darstellung ihrer Praktiken auf eine eigene allgemeine Deutung von Prävention beziehen, die im Rekurs auf kriminalpolitische Programmatik irritiert wird. So stehen sich zwar grundsätzlich zwei Deutungsweisen von Prävention gegenüber, wobei sich jedoch Tendenzen dahingehend zeigen, dass die den Fachpersonen eigenen Präventionsverständnisse und -konzepte durch von außen an sie herangetragene Erwartungen überlagert werden. Auch Jugendgewalt selbst sehen die Fachpersonen nicht als primäres Problem an, an dem sie in ihren eigenen, »sozialen« Präventionskonzepten ansetzen. Vielmehr problematisieren sie, insbesondere im Kontext der Jugendarbeit, öffentliche, mediale und politische Bearbeitungsweisen von Jugendgewalt, auf die sie mit offenen und ganzheitlichen Konzepten, die Jugendlichen Lern- und Bildungsprozesse im Sinne allgemeiner persönlicher Entwicklung ermöglichen sollen, reagieren. Eine Transformation von Jugendgewaltprävention, so ist die abschließende These der hier vorliegenden Arbeit, führt zu einer Vereinheitlichung verschiedener Verständnisse von (Jugendgewalt-)Prävention, die letztlich weniger individuelle Jugendliche in ihren je konkreten Lebenslagen adressiert, als darauf abzielt, Jugendgewalt als Problem zu normalisieren und damit für Bearbeitungsweisen dauerhaft zu reproduzieren und als Problembereich zu etablieren. 



\section{Zum Gebrauch von Prävention}

\subsection{Paradoxien im Kontext von Jugendgewaltprävention}

Die vorliegende Arbeit weist auf Paradoxien hin: Sie beobachtet, dass Jugendgewaltprävention selbst paradox ist, indem sie das hervorbringt, was sie an sich eigentlich vermeiden will (vgl. Brüchert 2010: 273; Schreiber 2011: 149f.). Jugendgewaltprävention rekonstruiert Jugendgewalt diskursiv und institutionalisiert und etabliert sie als zu bearbeitendes Problem. Dies ist bemerkenswert und kann in der hier vorliegenden Arbeit nachvollzogen werden. Bemerkenswert ist auch, dass sich diese Paradoxien in den Perspektiven der Fachpersonen widerspiegeln. Im Kontext einer »Verwissenschaftlichung des Sozialen « (Raphael 1996: 166)

"korrespondieren der Vielfalt der Ziele [von Subjektivierungsregimen] nicht minder heterogene Expertengruppen mit je spezifischen Wissensressourcen, Legitimationen und Kulturen. Subjektivierungsregime brauchen Subjektivierungsregisseure. Sie verleihen den Programmen Autorität, sie definieren die Aufgaben, vermitteln die Technologien zu ihrer Lösung, sie motivieren und sanktionieren, sie geben Feedbacks und evaluieren schließlich die Ergebnisse. [...] In der Gestalt des Experten radikalisiert sich das Paradox der Subjektkonstitution zum performativen Widerspruch: Einerseits tritt der Experte im appellativen Gestus einer Autorität auf, die weiß, was gut ist für die, zu denen er spricht. Andererseits nährt er das Misstrauen gegenüber jedweder Fremdbestimmung und predigt nichts als ,Werde du selbst! ‘." (Bröckling 2007: 41f.)

Dieses Paradox, das die Fachpersonen im Kontext von Jugendgewaltprävention tendenziell durchaus reflektieren, weil es sie in Dilemmata bringt, übertragen sie, so die These der hier vorliegenden Arbeit, auf die Adressatinnen und Adressaten ihrer Fachbereiche. Einerseits adressieren sie Jugendliche aus einer defizitorientierten Perspektive als nicht voll zurechnungsfähig hinsichtlich ihres Handelns und gar als »Opfer« gesellschaftlicher Skandalisierungsprozesse, andererseits überschreiben sie ihnen ein hohes Maß an Selbstverantwortlichkeit in Bezug auf ihre Lebensverläufe. Bühler-Niederberger spricht in diesem Kontext und in Bezug auf Kindheit betreffend von der 
"Macht der Unschuld [...], die Natur und Heiligkeit als ihre Referenzen beansprucht. In ihrem Einsatz, ihrer Wirkung und auch in ihrer keineswegs unschuldigen Konstruktion ist sie jedoch ganz von dieser Welt: Sie ist eine gesellschaftliche Macht. Damit ergibt sich auch ein Paradox: eine gesellschaftliche Macht beruht auf Kindern, die doch ihrerseits als Gruppe an der Gestaltung der sozialen Welt kaum beteiligt werden - [...] Gesellschaftliche Marginalisierung auf der einen Seite und moralische Überhöhung auf der anderen Seite gehen miteinander einher." (Bühler-Niederberger 2005: 9)

Dieser »Blick« trifft auch auf die dieser Arbeit vorliegenden Äußerungen über (die) Jugendliche(n) zu. Die Jugendlichen seien eigentlich »gut«, ja, sogar »vorzüglich«, gleichzeitig aber nicht ernst zu nehmen in ihren Artikulationen, Möglichkeiten und Reflexion ihrer Mitgestaltung und teilweise sogar eine bedrohliche und anonyme Masse. Im Bestreben, über Jugendgewaltprävention Jugendliche regierbar zu machen und damit sich und seine »Professionen« $\mathrm{zu}$ sichern, zielt »der Blick auf Jugendliche auf die Gesellschaft«. Der Gebrauch von Prävention, d.h. die Orientierung an damit einhergehenden anerkannten Wissensformen, ist in diesem Sinne als Herrschaftstechnik anzusehen, die Subjekte hervorbringt (vgl. Alkemeyer/Villa 2010: 315). Sie ist damit eine moderne Form von Herrschaft, die durch Paradoxien charakterisiert ist.

\subsection{Kritik einer Gesellschaft der Bestandssicherung}

Jugendgewaltprävention zielt den hier vorliegenden Ergebnissen nach nicht primär auf Vorbeugung im Sinne einer Reduktion von Jugendgewalt in der Zukunft, sondern auf Vorbeugung von Veränderung gesellschaftlicher Machtund Herrschaftsverhältnisse. Dies geschieht über die Rekonstruktion von Jugendgewalt als - aus primär kriminologischer Perspektive - problematisches Deutungsmuster. Jugendgewaltprävention ist hier als »kriminalpolitische Kontrollform « (Hess 2007: 6) zu verstehen, die ein vermeintliches Sicherheitsbedürfnis einer Mehrheitsgesellschaft befriedigen will, zulasten individueller Jugendlicher und einer Vielfalt an Maßnahmen im Kontext Sozialer Arbeit. Die Legitimation von Jugendgewaltprävention benötigt dabei die Reproduktion von Sicherheitsbedürfnissen über die Möglichkeit, Jugendgewalt dauerhaft zu problematisieren. Aus der Perspektive kriminalpolitischer Programmatik kann dies einen Ausschluss von Fachpersonen bedeuten, die sich in ihren Praktiken im Kontext von Jugendgewaltprävention nicht an den auf kriminalpolitischer Ebene relevant gesetzten Zielen orientieren. Es kann aber zur Folge haben, dass Jugendliche von der Möglichkeit ausgeschlossen werden, sich an Prozessen der Bildung und Sozialisation sowie an politischen Prozessen zu beteiligen. Aus der Perspektive der hier befragten Fachpersonen ist eine Transformation dieser Ziele auf der einen Seite sinnvoll - sofern sie sich über den 
Gebrauch von Jugendgewaltprävention und über das in diesem Zusammenhang relevant gesetzte, primär kriminologische Wissen professionalisieren können; auf der anderen Seite stellt eine Transformation dieser Ziele eigene Problemdeutungen und Präventionskonzepte infrage. Diese Ambivalenz wird austariert, indem - so eine der zentralen Thesen der vorliegenden Arbeit - die Fachpersonen die ihnen zugeschriebene Verantwortung auf Jugendliche übertragen. In der Konsequenz kann dies auch einen Ausschluss von Jugendlichen auf der Ebene der Praktiken Sozialer Arbeit bedeuten, wie die - vielleicht ein Extrembeispiel - Erzählung von Frau Blaum veranschaulicht. Die hier vorliegende Arbeit beschreibt eine Gesellschaft, die primär an der Sicherung des Status quo interessiert ist und Prävention als eine Praktik gebraucht, um etablierte Macht- und Herrschaftsverhältnisse zu sichern. Sozialpolitische Interessen werden dabei durch eine an Ausschluss interessierte Politik überformt. Dies wird sehr deutlich daran, dass das »Nationale Präventionsprogramm Jugend und Gewalt« primär durch das Bundesamt für Sozialversicherungen gefördert wird, was zunächst die Erwartung weckt, dass sozialpolitische Fragen, wie etwa Fragen der Integration und Inklusion, der Umverteilung oder der Verbesserung individueller Lebenschancen, bearbeitet werden. Im Vordergrund von Jugendgewaltprävention in der Schweiz steht jedoch keineswegs die soziale Sicherung von Individuen, sondern die Sicherung etablierter »Professionen« und politischer Apparate, die stellvertretend für die Mehrheitsgesellschaft stehen, indem eine bestimmte Bevölkerungsgruppe kriminalisiert wird. Insgesamt stellt sich die Frage, inwiefern für eine solche Sozialpolitik, die sich an Risiken orientiert, Ziele der Integration und Inklusion, der Umverteilung oder der Verbesserung individueller Lebenschancen überhaupt realistisch sind? Kann aktuelle Sozialpolitik auch anerkennungstheoretisch gedacht werden, was sowohl die Fachbereiche Sozialer Arbeit und die Anerkennung unterschiedlicher Projekte und Maßnahmen jenseits von »objektiver« Messbarkeit als auch die Anerkennung von Individuen betrifft? Die hier vorliegende Arbeit zeigt, dass Prävention denkbar ist als »ganzheitliches« Konzept. Als solches bezieht es sich auf Individuen in ihrer je spezifischen Lebenslage und kann als »integrativer« Bestandteil einer Sozialpolitik verstanden werden. Jugendgewalt wird aus der Perspektive der befragten Fachpersonen problematisiert, sie dient ihnen aber im eigenen Sinne nicht als Ausgangspunkt für Prävention.

„Präventionsarbeit an sich gibt es nicht. Was hingegen existiert, sind verschiedenste Ansätze Sozialer Arbeit, die wiederum präventiv orientiert sind. [...] Ganzheitlich orientierte Präventionsarbeit biete damit durch ihre konkrete Erfassung des Adressaten eine gute Möglichkeit, um frühzeitig auf sich eventuell weiter ergebende Problemfelder entsprechendes Einwirken wie auch Vorbeugen zu ermöglichen, um so die Handlung vor der Problementfaltung erheblich zu erleichtern." (Rapetti 2016: 37ff.) 
Zwar zeigt sich, dass die Fachpersonen normative Erwartungen an Individuen haben, jedoch auch, dass sie diese durchaus reflektieren. So ist denkbar, dass sie in ihrem Handeln nicht nur an ihren eigenen Normalitätsvorstellungen ansetzen, sondern die Normalitätskonzepte ihrer jeweiligen Adressatinnen und Adressaten miteinbeziehen. Hierin sollten die Fachpersonen bestärkt werden, damit Prävention nicht zu einer bloßen Anpassungserwartung verkommt, sondern zu einem ernst zu nehmenden Konzept einer ebenso ernst zu nehmenden Sozialpolitik wird. 


\section{Literatur}

Adler, Benjamin (2009): »Verantwortung - Kollektive als Handlungssubjekte«, in: Markus Christen/Max Baumann (Hg.), Verantwortung im politischen Diskurs, Dialog Ethik, Basel: EHM Schweizerischer Ärzteverlag, S. 87-101. Agamben, Giorgio (2008): Was ist ein Dispositiv?, Zürich/Berlin: Diaphanes. Albrecht, Günter (Hg.) (2012): Handbuch soziale Probleme, Wiesbaden: VS. Alkemeyer, Thomas/Villa, Paula-Irene (2010): »Somatischer Eigensinn? Kritische Anmerkungen zu Diskurs- und Gouvernementalitätsforschung aus subjektivationstheoretischer und praxeologischer Perspektive«, in: Johannes Angermüller/Silke van Dyk (Hg.), Diskursanalyse meets Gouvernementalitätsforschung. Perspektiven auf das Verhältnis von Subjekt, Sprache, Macht und Wissen, Frankfurt a.M.: Campus, S. 315-335.

Althoff, Martina (2002a): »Kriminalität - eine diskursive Praxis. Eine Einführung in die Diskursanalyse Michel Foucaults«, in: Roland Anhorn (Hg.), Kritische Kriminologie und soziale Arbeit. Impulse für professionelles Selbstverständnis und kritisch-reflexive Handlungskompetenz, Weinheim: Juventa, S. 47-73.

Althoff, Martina (2002b): »Jugendkriminalität und Gewalt. Einige Überlegungen zur öffentlichen Thematisierung von Jugend«, in: Frank Bettinger (Hg.), Gefährdete Jugendliche? Jugend, Kriminalität und der Ruf nach Strafe, Opladen: Leske + Budrich, S. 75-88.

Althusser, Louis (1977): Ideologie und ideologische Staatsapparate. Aufsätze zur marxistischen Theorie, Hamburg/Berlin: VSA.

Angermüller, Johannes (2014): »Einleitung. Diskursforschung als Theorie und Analyse. Umrisse eines interdisziplinären und internationalen Feldes«, in: Johannes Angermüller/Martin Nonhoff/Eva Herschinger (Hg.), Diskursforschung. Ein interdisziplinäres Handbuch, Bielefeld: transcript, S. 16-36. Angermüller, Johannes/van Dyk, Silke (Hg.) (2010): Diskursanalyse meets Gouvernementalitätsforschung. Perspektiven auf das Verhältnis von Subjekt, Sprache, Macht und Wissen, Frankfurt a.M.: Campus.

Angermüller, Johannes/Nonhoff, Martin/Herschinger, Eva (Hg.) (2014): Diskursforschung. Ein interdisziplinäres Handbuch, Bielefeld: transcript. 
Angermüller, Johannes/Wedl, Juliette (2014): »Diskursforschung in der Soziologie«, in: Johannes Angermüller/Martin Nonhoff/Herschinger (Hg.), Diskursforschung. Ein interdisziplinäres Handbuch, Bielefeld: transcript, S. 162-191.

Anhorn, Roland (Hg.) (2002): Kritische Kriminologie und soziale Arbeit. Impulse für professionelles Selbstverständnis und kritisch-reflexive Handlungskompetenz, Weinheim: Juventa.

Anhorn, Roland (2002): »Jugend - Abweichung - Drogen: Zur Konstruktion eines sozialen Problems«, in: Frank Bettinger (Hg.), Gefährdete Jugendliche? Jugend, Kriminalität und der Ruf nach Strafe, Opladen: Leske + Budrich, S. 47-74.

Anhorn, Roland (2008): »Zur Einleitung: Warum sozialer Ausschluss für Theorie und Praxis Sozialer Arbeit zum Thema werden muss «, in: Roland Anhorn/Frank Bettinger/Johannes Stehr (Hg.), Sozialer Ausschluss und soziale Arbeit. Positionsbestimmungen einer kritischen Theorie und Praxis sozialer Arbeit, Wiesbaden: VS, S. 13-48.

Anhorn, Roland/Bettinger, Frank (2002): »Zum Nutzen der Kritischen Kriminologie: Bausteine einer Theorie und Praxis Kritischer Sozialer Arbeit«, in: Roland Anhorn (Hg.), Kritische Kriminologie und soziale Arbeit. Impulse für professionelles Selbstverständnis und kritisch-reflexive Handlungskompetenz, Weinheim: Juventa, S. 223-257.

Anhorn, Roland/Bettinger, Frank/Stehr, Johannes (Hg.) (2007): Foucaults Machtanalytik und Soziale Arbeit. Eine kritische Einführung und Bestandsaufnahme, Wiesbaden: VS.

Anhorn, Roland/Bettinger, Frank/Stehr, Johannes (Hg.) (2008): Sozialer Ausschluss und soziale Arbeit. Positionsbestimmungen einer kritischen Theorie und Praxis sozialer Arbeit, Wiesbaden: VS.

Arbeitskreis junger Kriminologen (Hg.) (1986): Kritische Kriminologie heute. 1. Beiheft des Kriminologischen Journals, Weinheim: Juventa.

Autrata, Otger/Scheu, Bringfriede (Hg.) (2009): Jugendgewalt. Interdisziplinäre Sichtweisen, Wiesbaden: VS.

Balz, Hans-Jürgen/Benz, Benjamin/Kuhlmann, Carola (Hg.) (2012): Soziale Inklusion. Grundlagen, Strategien und Projekte in der Sozialen Arbeit, Wiesbaden: VS.

Baratta, Alessandro (1986): »Soziale Probleme und Konstruktion der Kriminalität«, in: Arbeitskreis junger Kriminologen (Hg.), Kritische Kriminologie heute. 1. Beiheft des Kriminologischen Journals, Weinheim: Juventa, S. 200-218.

Bauman, Zygmunt (1992): Dialektik der Ordnung. Die Moderne und der Holocaust, Hamburg: Europäische Verlagsanstalt.

Becker, Howard S. (1963): Outsiders: Studies in the Sociology of Deviance, New York: The Free Press. 
Becker, Ruth/Kortendiek, Beate (Hg.) (2010): Handbuch Frauen- und Geschlechterforschung. Theorie, Methoden, Empirie, Wiesbaden: VS/GWV.

Bender, Désirée/Eck, Sandra (2014): »Studentische Subjektivierungsweisen im Machtnetz des Bologna-Prozesses. Eine Dispositivanalyse narrativer Interviews«, in: Johannes Angermüller/Martin Nonhoff/Eva Herschinger (Hg.), Diskursforschung. Ein interdisziplinäres Handbuch, Bielefeld: transcript, S. 472-499.

Benjamin, Walter (2006): Zur Kritik der Gewalt und andere Aufsätze, Frankfurt a.M.: Suhrkamp.

Benz, Benjamin/Rieger, Günter/Schönig, Werner/Többe-Schukalla, Monika (Hg.) (2013): Politik Sozialer Arbeit. Band 1: Grundlagen, theoretische Perspektiven und Diskurse, Weinheim: Beltz Juventa.

Benz, Benjamin/Rieger, Günter/Schönig, Werner/Többe-Schukalla, Monika (2013): »Die Politik Sozialer Arbeit - Umrisse, Gegenstände und Positionen«, in: Dies. (Hg.), Politik Sozialer Arbeit. Band 1: Grundlagen, theoretische Perspektiven und Diskurse, Weinheim: Beltz Juventa, S. 8-29.

Bergmann, Jörg (1981): »Ethnomethodologische Konversationsanalyse«, in: Peter Schröder/Hugo Steger (Hg.), Dialogforschung. Jahrbuch 1980 des Instituts für deutsche Sprache, Düsseldorf: Pädagogischer Verlag Schwann, S. 9-52.

Bergmann, Jörg (1985): »Flüchtigkeit und methodische Fixierung sozialer Wirklichkeit«, in: Wolfgang Bonß/Heinz Hartmann (Hg.), Entzauberte Wissenschaft, Göttingen: Otto Schwarz \& Co, S. 299-320.

Bericht des Bundesrates (2009): Jugend und Gewalt. Wirksame Prävention in den Bereichen Familie, Schule, Sozialraum und Medien. Bericht des Bundesrates in Erfüllung der Postulate Leuthard (03.3298) vom 17. Juni 2003, Amherd (06.3646) vom 6. Dezember 2006 und Galladé (07.3665) vom 4. Oktober 2007, www.news.admin.ch/NSBSubscriber/message/attach ments/15741.pdf vom 12.01.2017.

Bericht des Bundesrates (2015): Jugend und Gewalt. Stand der Prävention und Zusammenwirken mit Intervention und Repression, https://www.newsd. admin.ch/newsd/message/attachments/39408.pdf vom 12.01.2017.

Berner, Frank/Groenemeyer, Axel (2003): »Kriminalpolitische Diskurse und die Institutionalisierung kommunaler Kriminalprävention«, in: Axel Groenemeyer (Hg.), Soziale Probleme und politische Diskurse. Konstruktionen von Kriminalpolitik in sozialen Kontexten, Bielefeld: Universität Bielefeld, S. 85-114.

Bettinger, Frank (Hg.) (2002): Gefährdete Jugendliche? Jugend, Kriminalität und der Ruf nach Strafe, Opladen: Leske + Budrich.

Bettinger, Frank (2007): »Diskurse - Konstitutionsbedingung des Sozialen«, in: Roland Anhorn/Frank Bettinger/Johannes Stehr (Hg.), Foucaults 
Machtanalytik und Soziale Arbeit. Eine kritische Einführung und Bestandsaufnahme, Wiesbaden: VS, S. 75-90.

Bock, Karin/Miethe, Ingrid/Ritter, Bettina (Hg.) (2010): Handbuch qualitative Methoden in der sozialen Arbeit, Opladen: Budrich.

Böllinger, Lorenz (2010): »Können Menschenbilder von >gefährlichen Menschen selbst gefährlich werden?«, in: Lorenz Böllinger/Michael Jasch/ Susanne Krasmann/Arno Pilgram/Cornelius Prittwitz/Herbert Reinke/ Dorothea Rzepka (Hg.), Gefährliche Menschenbilder. Biowissenschaften, Gesellschaft und Kriminalität, Baden-Baden: Nomos, S. 13-33.

Böllinger, Lorenz/Jasch, Michael/Krasmann, Susanne/Pilgram, Arno/Prittwitz, Cornelius/Reinke, Herbert/Rzepka, Dorothea (Hg.) (2010): Gefährliche Menschenbilder. Biowissenschaften, Gesellschaft und Kriminalität, Baden-Baden: Nomos.

Bogner, Alexander (Hg.) (2009): Experteninterviews. Theorien, Methoden, Anwendungsfelder, Wiesbaden: VS.

Bogner, Alexander/Menz, Wolfgang (2009): »Das theoriegenerierende Experteninterview. Erkenntnisinteresse, Wissensformen, Interaktion«, in: Alexander Bogner (Hg.), Experteninterviews. Theorien, Methoden, Anwendungsfelder, Wiesbaden: VS, S. 61-97.

Bohnsack, Ralf (2001): »Typenbildung, Generalisierung und komparative Analyse: Grundprinzipien der dokumentarischen Methode«, in: Ralf Bohnsack/Iris Nentwig-Gesemann/Arnd-Michael Nohl (Hg.), Die dokumentarische Methode und ihre Forschungspraxis. Grundlagen qualitativer Sozialforschung, Opladen: Leske + Budrich, S. 225-252.

Bohnsack, Ralf (2003): Rekonstruktive Sozialforschung. Einführung in qualitative Methoden, Opladen: Leske + Budrich.

Bohnsack, Ralf/Nentwig-Gesemann, Iris/Nohl, Arnd-Michael (Hg.) (2001): Die dokumentarische Methode und ihre Forschungspraxis. Grundlagen qualitativer Sozialforschung, Opladen: Leske + Budrich.

Bohnsack, Ralf/Nentwig-Gesemann, Iris/Nohl, Arnd-Michael (2001): »Einleitung: Die dokumentarische Methode und ihre Forschungspraxis«. in: Dies. (Hg.), Die dokumentarische Methode und ihre Forschungspraxis. Grundlagen qualitativer Sozialforschung, Opladen: Leske + Budrich, S. 9-24.

Bonß, Wolfgang/Hartmann, Heinz (Hg.) (1985): Entzauberte Wissenschaft, Göttingen: Otto Schwarz \& Co.

Bourdieu, Pierre (1997): »Die männliche Herrschaft«, in: Irene Dölling/Beate Krais (Hg.), Ein alltägliches Spiel. Geschlechterkonstruktion in der sozialen Praxis, Frankfurt a.M.: Suhrkamp, S. 153-216.

Breidenstein, Georg/Kelle, Helga (1998): Geschlechteralltag in der Schulklasse. Ethnographische Studien zur Gleichaltrigenkultur, Weinheim/München: Juventa. 
Bröckling, Ulrich (2002): »Die Macht der Vorbeugung. 16 Thesen zur Prävention«, in: Widersprüche 22 (86), S. 39-52.

Bröckling, Ulrich (2007): Das unternehmerische Selbst. Soziologie einer Subjektivierungsform, Frankfurt a.M.: Suhrkamp.

Bröckling, Ulrich (2009): »Prävention«, in: Ulrich Bröckling/Susanne Krasmann/Thomas Lemke (Hg.), Glossar der Gegenwart, Frankfurt a.M.: Suhrkamp, S. 210-214.

Bröckling, Ulrich (2013): »Anrufung und Adresse«, in: Andreas Gelhard/Thomas Alkemeyer/Norbert Ricken (Hg.), Techniken der Subjektivierung, München: Wilhelm Fink, S. 49-59.

Bröckling, Ulrich/Krasmann, Susanne (2010): »Ni méthode, ni approche. Zur Forschungsperspektive der Gouvernementalitätsstudien - mit einem Seitenblick auf Konvergenzen und Divergenzen zur Diskursforschung«, in: Johannes Angermüller/Silke van Dyk (Hg.), Diskursanalyse meets Gouvernementalitätsforschung. Perspektiven auf das Verhältnis von Subjekt, Sprache, Macht und Wissen, Frankfurt a.M.: Campus, S. 23-42.

Bröckling, Ulrich/Krasmann, Susanne/Lemke, Thomas (Hg.) (2009): Glossar der Gegenwart, Frankfurt a.M.: Suhrkamp.

Brüchert, Oliver (2010): »>Gewalt ist keine Lösungく. Der Beitrag von Kampagnen der Kriminalprävention zur Konstruktion sozialer Probleme«, in: Axel Groenemeyer (Hg.), Doing Social Problems. Mikroanalysen der Konstruktion sozialer Probleme und sozialer Kontrolle in institutionellen Kontexten, Wiesbaden: VS, S. 272-295.

Bublitz, Hannelore (Hg.) (1998): Das Geschlecht der Moderne. Genealogie und Archäologie der Geschlechterdifferenz, Frankfurt/New York: Campus.

Bublitz, Hannelore/Bührmann, Andrea D./Hanke, Christine/Seier, Andrea (Hg.) (1999): Das Wuchern der Diskurse. Perspektiven der Diskursanalyse Foucaults, Frankfurt a.M./New York: Campus.

Bublitz, Hannelore/Bührmann, Andrea D./Hanke, Christine/Seier, Andrea (1999): »Diskursanalyse - (k)eine Methode? Eine Einleitung«, in: Dies. (Hg.), Das Wuchern der Diskurse. Perspektiven der Diskursanalyse Foucaults, Frankfurt a.M./New York: Campus, S. 10-21.

Bühler-Niederberger, Doris (Hg.) (2005): Macht der Unschuld. Das Kind als Chiffre, Wiesbaden: VS.

Bühler-Niederberger, Doris (2005): »Einleitung: Der Blick auf das Kind - gilt der Gesellschaft«, in: Dies. (Hg.), Macht der Unschuld. Das Kind als Chiffre, Wiesbaden: VS, S. 9-22.

Bühler-Niederberger, Doris (2007): The power of innocence. Social politics for childrenbetweenseparationand participation. WorkingPapers Series 4/2007, http://institutinfancia.cat/wp-content/uploads/2016/08/2007_Thepower-of-innocence_article_angl.pdf vom 12.01.2017. 
Bühler-Niederberger, Doris (2010): »Organisierte Sorge für Kinder, Eigenarten und Fallstricke - eine generationale Perspektive«, in: Doris Bühler-Niederberger/Johanna Mierendorff/Andreas Lange (Hg.), Kindheit zwischen fürsorglichem Zugriff und gesellschaftlicher Teilhabe, Wiesbaden: VS, S. 1741.

Bühler-Niederberger, Doris (2011): Lebensphase Kindheit. Theoretische Ansätze, Akteure und Handlungsräume, Weinheim/München: Juventa.

Bühler-Niederberger, Doris/Mierendorff, Johanna/Lange, Andreas (Hg.) (2010): Kindheit zwischen fürsorglichem Zugriff und gesellschaftlicher Teilhabe, Wiesbaden: VS.

Bührmann, Andrea D. (1997): »Geschlecht als Dispositiv«, in: Andreas Disselnkötter (Hg.), Evidenzen im Fluss. Demokratieverluste in Deutschland: Modell D, Geschlechter, Rassismus, PC, Duisburg: Diss, S. 135-152.

Bührmann, Andrea D. (1998): »Die Normalisierung der Geschlechter in Geschlechterdispositiven«, in: Hannelore Bublitz (Hg.), Das Geschlecht der Moderne. Genealogie und Archäologie der Geschlechterdifferenz, Frankfurt/New York: Campus, S. 71-94.

Bührmann, Andrea D. (1999): »Der Diskurs als Diskursgegenstand im Horizont der kritischen Ontologie der Gegenwart«, in: Hannelore Bublitz/Andrea D. Bührmann/Christine Hanke/Andrea Seier (Hg.), Das Wuchern der Diskurse. Perspektiven der Diskursanalyse Foucaults, Frankfurt a.M./New York: Campus, S. 49-62.

Bührmann, Andrea D./Schneider, Werner (2008): Vom Diskurs zum Dispositiv. Eine Einführung in die Dispositivanalyse, Bielefeld: transcript.

Bührmann, Andrea D./Schneider, Werner (2010): »Die Dispositivanalyse als Forschungsperspektive. Begrifflich-konzeptionelle Überlegungen zur Analyse gouvernementaler Taktiken und Technologien«, in: Johannes Angermüller/Silke van Dyk (Hg.), Diskursanalyse meets Gouvernementalitätsforschung. Perspektiven auf das Verhältnis von Subjekt, Sprache, Macht und Wissen, Frankfurt a.M.: Campus, S. 261-288.

Butler, Judith (2001): Psyche der Macht. Das Subjekt der Unterwerfung, Frankfurt a.M.: Suhrkamp.

Butler, Judith (2006): Hass spricht. Zur Politik des Performativen, Frankfurt a.M.: Suhrkamp.

Caplan, Gerald (1964): Principles of Preventive Psychiatry, New York: Basic Books.

Castel, Robert (1983): »Von der Gefährlichkeit zum Risiko«, in: Manfred Max Wambach (Hg.), Der Mensch als Risiko. Zur Logik von Prävention und Früherkennung, Frankfurt a.M.: Suhrkamp, S. 51-74.

Christen, Markus/Baumann, Max (Hg.) (2009): Verantwortung im politischen Diskurs. Dialog Ethik, Basel: EMH Schweizerischer Ärzteverlag. 
Cremer-Schäfer, Helga (1995): »Skandalisierungsfallen«, in: Kriminologisches Journal 27 (1), S. 23-36.

Cremer-Schäfer, Helga (1998): »Die Gewalt, die sozialen Probleme und die Skandalisierungsfalle«, in: Helga Cremer-Schäfer/Heinz Steinert (Hg.): Straflust und Repression. Zur Kritik der populistischen Kriminologie, Münster: Westfälisches Dampfboot, S. 118-135.

Cremer-Schäfer, Helga (2016): »Über die Produktivität der >frühen Kritikくvon Prävention als Denkweise und Politik«, in: Widersprüche 36 (139), S. 11-23.

Cremer-Schäfer, Helga/Steinert, Heinz (Hg.) (1998): Straflust und Repression. Zur Kritik der populistischen Kriminologie, Münster: Westfälisches Dampfboot.

Collins, Randall (2011): Dynamik der Gewalt. Eine mikrosoziologische Theorie, Hamburg: Hamburger Edition.

Deleuze, Gilles (1992): Foucault, Frankfurt a.M.: Suhrkamp.

Denninger, Erhard (1988): »Der Präventions-Staat«, in: Kritische Justiz 21 (1), S. 1-15.

Disselnkötter, Andreas (Hg.) (1997): Evidenzen im Fluss. Demokratieverluste in Deutschland: Modell D, Geschlechter, Rassismus, PC, Duisburg: DISS.

Dollinger, Bernd (2006): »Prävention. Unintendierte Nebenfolgen guter Absichten«, in: Bernd Dollinger/Jürgen Raithel (Hg.), Aktivierende Sozialpädagogik. Ein kritisches Glossar, Wiesbaden: VS, S. 145-154.

Dollinger, Bernd (2015): »Risiken (in) der Sozialpädagogik. Anmerkungen zur sozialpädagogischen Dimension von Risikosemantiken«, in: Bernd Dollinger/Axel Groenemeyer/Dorothea Rzepka (Hg.), Devianz als Risiko. Neue Perspektiven des Umgangs mit abweichendem Verhalten, Delinquenz und sozialer Auffälligkeit, Weinheim: Beltz Juventa, S. 45-59.

Dollinger, Bernd/Groenemeyer, Axel/Rzepka, Dorothea (Hg.) (2015): Devianz als Risiko. Neue Perspektiven des Umgangs mit abweichendem Verhalten, Delinquenz und sozialer Auffälligkeit, Weinheim: Beltz Juventa.

Dollinger, Bernd/Oelkers, Nina (Hg.) (2015): Sozialpädagogische Perspektiven auf Devianz, Weinheim: Beltz Juventa.

Dollinger, Bend/Oelkers, Nina (2015): »Zur Einleitung: Sozialpädagogische Perspektiven auf Devianz«, in: Dies. (Hg.), Sozialpädagogische Perspektiven auf Devianz, Weinheim: Beltz Juventa, S. 9-32.

Dollinger, Bernd/Raithel, Jürgen (Hg.) (2006): Aktivierende Sozialpädagogik. Ein kritisches Glossar, Wiesbaden: VS.

Dollinger, Bernd/Schmidt-Semisch, Henning (Hg.) (2011): Handbuch Jugendkriminalität. Kriminologie und Sozialpädagogik im Dialog, Wiesbaden: VS.

Dollinger, Bernd/Schmid-Semisch Henning (2011): »Sozialpädagogik und Kriminologie im Dialog. Einführende Perspektiven zum Ereignis >Jugend- 
kriminalität«, in: Dies., Handbuch Jugendkriminalität. Kriminologie und Sozialpädagogik im Dialog, Wiesbaden: VS, S. 11-21.

Dölling, Irene/Krais, Beate (Hg.) (1997): Ein alltägliches Spiel. Geschlechterkonstruktion in der sozialen Praxis, Frankfurt a.M.: Suhrkamp.

Donzelot, Jaques (1980): Die Ordnung der Familie, Frankfurt a.M.: Suhrkamp.

Dreyfus, Hubert/Rabinow, Paul/Foucault, Michel (Hg.) (1994): Michel Foucault. Jenseits von Strukturalismus und Hermeneutik, Weinheim: BeltzAthenäum.

Drilling, Matthias (Hg.) (2002): Gewalt an Schulen: Ursachen, Prävention, Intervention. Beiträge der 2. Nationalen Fachtagung der Stiftung Erziehung zur Toleranz, Zürich: Pestalozzianum.

EDI (Eidgenössisches Departement des Inneren)/BSV (Bundesamt für Sozialversicherungen) (2010a): Gesamtschweizerisches Präventionsprogramm Jugend und Gewalt, https://www.newsd.admin.ch/newsd/message/attach ments/19465.pdf vom 12.01.2017.

EDI (Eidgenössisches Departement des Inneren)/BSV (Bundesamt für Sozialversicherungen) (2010b): Nationales Programm Jugendmedienschutz und Medienkompetenzen, www.news.admin.ch/NSBSubscriber/message/ attachments/19468.pdf vom 12.01.2017.

Eisenstadt, Samuel N. (1966): Von Generation zu Generation. Altersgruppen und Sozialstruktur, München: Juventa.

Elliott, Anthony (Hg.) (2011): Routledge Handbook of identity studies, London: Routledge.

Erikson, Erik H. (1970): Identität und Lebenszyklus. 3 Aufsätze, Frankfurt a.M.: Suhrkamp.

Felten, Mirjam von (2000): »... aber das ist noch lange nicht Gewalt«. Empirische Studie zur Wahrnehmung von Gewalt bei Jugendlichen, Opladen: Leske + Budrich .

Feustel, Robert/Keller, Reiner/Schrage, Dominik/Wedl, Juliette/Wrana, Daniel/van Dyk, Silke (2014): »Zur method(olog)ischen Systematisierung der sozialwissenschaftlichen Diskursforschung. Herausforderung, Gratwanderung, Kontroverse. Eine Debatte«, in: Johannes Angermüller/Martin Nonhoff/Eva Herschinger (Hg.), Diskursforschung. Ein interdisziplinäres Handbuch, Bielefeld: transcript, S. 482-506.

Féraud, Marius/Huegli, Eveline (Büro Vatter) (2015): Schlussevaluation. Gesamtschweizerisches Präventionsprogramm Jugend und Gewalt. Schlussbericht. BBL, Bern, http://www.jugendundgewalt.ch/uploads/media/715d_eBericht_Schlussevaluation.pdf vom 12.01.2017.

Finkel, Roland (1995): »Kriminalitätsverhütung als gesamtgesellschaftliche Aufgabe. Kommunale Präventionsräte als Kriminalpolitisches Konzept am Beispiel Schleswig-Holsteins«, in: Rolf Gössner (Hg.), Mythos Sicherheit. 
Der hilflose Schrei nach dem starken Staat, Baden-Baden: Nomos, S. 415427 .

Foucault, Michel (1977): Überwachen und Strafen. Die Geburt des Gefängnisses, Frankfurt a.M.: Suhrkamp.

Foucault, Michel (1979): Sexualität und Wahrheit 1. Der Wille zum Wissen, Frankfurt a.M.: Suhrkamp.

Foucault, Michel (1986): Sexualität und Wahrheit 2. Der Gebrauch der Lüste, Frankfurt a.M.: Suhrkamp.

Foucault, Michel (1994a): Archäologie des Wissens, Frankfurt a.M.: Suhrkamp.

Foucault, Michel (1994b): »Das Subjekt und die Macht«, in: Hubert Dreyfus/ Paul Rabinow/Michel Foucault (Hg.), Michel Foucault. Jenseits von Strukturalismus und Hermeneutik, Weinheim: Beltz-Athenäum, S. 243-261.

Foucault, Michel (2004a): Sicherheit, Territorium, Bevölkerung. Geschichte der Gouvernementalität I. Vorlesung am Collège de France 1977-1978. Hg. v. Michel Sennelart, Frankfurt a.M.: Suhrkamp.

Foucault, Michel (2004b): Die Geburt der Biopolitik. Geschichte der Gouvernementalität II. Vorlesung am Collège de France 1978-1979. Hg. v. Michel Sennelart, Frankfurt a.M.: Suhrkamp.

Foucault, Michel/Defert, Daniel (Hg.) (2005): Schriften in vier Bänden, Frankfurt a.M.: Suhrkamp.

Gaffer, Yvonne/Liell, Christoph (2001): »Handlungstheoretische und methodologische Aspekte der dokumentarischen Interpretation jugendkultureller Praktiken«, in: Ralf Bohnsack/Iris Nentwig-Gesemann/Arnd-Michael Nohl (Hg.), Die dokumentarische Methode und ihre Forschungspraxis. Grundlagen qualitativer Sozialforschung, Opladen: Leske + Budrich, S. 179-203.

Galtung, Johan (1975): Strukturelle Gewalt. Beiträge zur Friedens- und Konfliktforschung, Reinbek bei Hamburg: Rowohlt.

Garland, David (2008): Kultur der Kontrolle. Verbrechensbekämpfung und soziale Ordnung in der Gegenwart, Frankfurt a.M./New York: Campus.

Gehring, Petra (2008): »Abseits des Akteurs-Subjekts. Selbsttechniken, Ethik als politische Haltung und der Fall der freimütigen Rede«, in: Reiner Keller/Werner Schneider/Willy Viehöver (Hg.), Diskurs, Macht, Subjekt, Wiesbaden: VS, S. 21-33.

Gelhard, Andreas/Alkemeyer, Thomas/Ricken, Norbert (Hg.) (2013): Techniken der Subjektivierung, München: Wilhelm Fink.

Glaser, Barney G./Strauss, Anselm L. (1979): »Die Entdeckung gegenstandbezogener Theorie«, in: Christel Hopf/Elmar Weingarten (Hg.), Qualitative Sozialforschung, Stuttgart: Klett-Cotta, S. 91-111.

Gössner, Rolf (Hg.) (1995): Mythos Sicherheit. Der hilflose Schrei nach dem starken Staat, Baden-Baden: Nomos. 
Goffman, Erving (1963): Stigma. Notes on the management of spoiled identity, Englewood Cliffs, N.J.: Prentice-Hall.

Griese, Hartmut M. (2012): »Jugend«, in: Günter Albrecht (Hg.), Handbuch soziale Probleme, Wiesbaden: VS, S. 692-715.

Griese, Hartmut M. (2014): »Jugend - immer noch ein soziales Problem? Persönliche Anmerkungen nach 30 Jahren«, in: Axel Groenemeyer/Dagmar Hoffmann (Hg.), Jugend als soziales Problem - soziale Probleme der Jugend? Diagnosen, Diskurse und Herausforderungen, Weinheim: Beltz-Juventa, S. 17-28.

Groenemeyer, Axel (Hg.) (2003): Soziale Probleme und politische Diskurse. Konstruktionen von Kriminalpolitik in sozialen Kontexten, Bielefeld: Universität Bielefeld.

Groenemeyer, Axel (Hg.) (2010): Doing Social Problems. Mikroanalysen der Konstruktion sozialer Probleme und sozialer Kontrolle in institutionellen Kontexten, Wiesbaden: VS.

Groenemeyer, Axel (2012): »Soziale Probleme - Fragestellungen, Konzepte und theoretische Perspektiven«, in: Günter Albrecht (Hg.), Handbuch soziale Probleme, Wiesbaden: VS, S. 17-116.

Groenemeyer, Axel (2014): »Jugend im Problemdiskurs - Probleme im Jugenddiskurs. Was bedeutet das Reden über Jugend?«, in: Axel Groenemeyer/ Dagmar Hoffmann (Hg.), Jugend als soziales Problem - soziale Probleme der Jugend? Diagnosen, Diskurse und Herausforderungen, Weinheim: Beltz-Juventa, S. 50-76.

Groenemeyer, Axel (2015): »Soziale Konstruktionen als Ordnungsstörungen. Abweichung von Risiko«, in: Bernd Dollinger/Axel Groenemeyer/Dorothea Rzepka (Hg.), Devianz als Risiko. Neue Perspektiven des Umgangs mit abweichendem Verhalten, Delinquenz und sozialer Auffälligkeit, Weinheim: Beltz Juventa, S. 9-43.

Groenemeyer, Axel/Hoffmann, Dagmar (Hg.) (2014): Jugend als soziales Problem - soziale Probleme der Jugend? Diagnosen, Diskurse und Herausforderungen, Weinheim: Beltz-Juventa.

Grunwald, Klaus/Thiersch, Hans (Hg.) (2004): Praxis lebensweltorientierter sozialer Arbeit. Handlungszugänge und Methoden in unterschiedlichen Arbeitsfeldern, Weinheim: Juventa.

Grunwald, Klaus/Thiersch, Hans (2004): »Das Konzept Lebensweltorientierte Soziale Arbeit - einleitende Bemerkungen«, in: Dies. (Hg.), Praxis lebensweltorientierter sozialer Arbeit. Handlungszugänge und Methoden in unterschiedlichen Arbeitsfeldern, Weinheim: Juventa, S. 13-39.

Haffke, Bernhard (2005): Vom Rechtsstaat zum Sicherheitsstaat?, in: Kritische Justiz 38 (1), S. 17-35.

Han, Byung-Chul (2010): Was ist Macht?, Stuttgart: Reclam.

Han, Byung-Chul (2011): Topologie der Gewalt, Berlin: Matthes \& Seitz Berlin. 
Helfferich, Cornelia (2011): Die Qualität qualitativer Daten. Manual für die Durchführung qualitativer Interviews, Wiesbaden: VS.

Hempel, Leon/Krasmann, Susanne/Bröckling, Ulrich (Hg.) (2011): Sichtbarkeitsregime. Überwachung, Sicherheit und Privatheit im 21. Jahrhundert, Wiesbaden: VS.

Hempel, Leon/Krasmann, Susanne/Bröckling, Ulrich (2011): »Sichtbarkeitsregime: Eine Einleitung«, in: Dies. (Hg.), Sichtbarkeitsregime. Überwachung, Sicherheit und Privatheit im 21. Jahrhundert, Wiesbaden: VS, S. 7-26.

Herrmann, Cora (2007): »Zur Transformation der Vorstellung von >guter Arbeit - Aneignungsweisen der Qualitätsdebatte in der stationären Kinder- und Jugendhilfe«, in: Roland Anhorn/Frank Bettinger/Johannes Stehr (Hg.), Foucaults Machtanalytik und Soziale Arbeit. Eine kritische Einführung und Bestandsaufnahme, Wiesbaden: VS, S. 295-308.

Hess, Henner (2007): »Einleitung. David Garlands >Culture of Control $<$ und die deutsche Kritische Kriminologie«, in: Henner Hess/Lars Ostermeier/Bettina Paul (Hg.), Kontrollkulturen. Texte zur Kriminalpolitik im Anschluss an David Garland. 9. Beiheft des Kriminologischen Journals, Weinheim: Juventa, S. 6-21.

Hess, Henner/Ostermeier, Lars/Paul, Bettina (Hg.) (2007): Kontrollkulturen. Texte zur Kriminalpolitik im Anschluss an David Garland. 9. Beiheft des Kriminologischen Journals, Weinheim: Juventa.

Hirschauer, Stefan (2001): »Ethnografisches Schreiben und die Schweigsamkeit des Sozialen. Zur Methodologie der Beschreibung«, in: Zeitschrift für Soziologie 30 (6), S. 429-451.

Hitzler, Ronald/Honer, Anne (Hg.) (1997): Sozialwissenschaftliche Hermeneutik. Eine Einführung, Opladen: Leske + Budrich.

Höllmüller, Hubert (2009): »Jugendgewalt aus sozialphilosophischer Perspektive«, in: Otger Autrata/Bringfriede Scheu (Hg.), Jugendgewalt. Interdisziplinäre Sichtweisen, Wiesbaden: VS, S. 51-75.

Holstein, James A./Miller, Gale (1993): »Social Constructivism and Social Problems Work«, in: Gale Miller/James A. Holstein (Hg.), Constructionist Controversies. Issues in Social Problems Theory, New York: Aldine de Gruyter, S. 131-151.

Hopf, Christel/Weingarten, Elmar (Hg.) (1979): Qualitative Sozialforschung, Stuttgart: Klett-Cotta.

Huber, Sven (2014): Zwischen den Stühlen. Mobile und aufsuchende Jugendarbeit im Spannungsfeld von Aneignung und Ordnungspolitik, Wiesbaden: VS.

Hugger, Paul (1995): »Elemente einer Kulturanthropologie«, in: Paul Hugger/ Heinz Bonfadelli/Ulrich Stadler (Hg.), Gewalt. Kulturelle Formen in Geschichte und Gegenwart, Zürich: Unionsverlag, S. 17-27. 
Hugger, Paul/Bonfadelli, Heinz/Stadler, Ulrich (Hg.) (1995): Gewalt. Kulturelle Formen in Geschichte und Gegenwart, Zürich: Unionsverlag.

Jordan, Erwin/Maykus, Stephan/Stuckstätte, Eva C. (2015): Kinder- und Jugendhilfe. Einführung in Geschichte und Handlungsfelder, Organisationsformen und gesellschaftliche Problemlagen, Weinheim/Basel: Beltz Juventa.

Jüttemann, Gerd (Hg.) (1989): Qualitative Forschung in der Psychologie, Heidelberg: Roland Asanger.

Kalthoff, Herbert (Hg.) (2008): Theoretische Empirie. Zur Relevanz qualitativer Forschung, Frankfurt a.M.: Suhrkamp.

Kappeler, Manfred (2016): »Prävention als Verhinderung selbstbestimmten Lebens in der Gegenwart im Namen der Zukunft«, in: Widersprüche 36 (139), S. 53-68.

Kelle, Helga (1996): »Kinder als Akteure: Ethnographische Ansätze in der Kindheitsforschung«, in: ZSE 16 (1), 47-67.

Keller, Reiner (1997): »Diskursanalyse«, in: Ronald Hitzler/Anne Honer (Hg.), Sozialwissenschaftliche Hermeneutik. Eine Einführung, Opladen: Leske + Budrich, S. 309-333.

Keller, Reiner (2001): »Wissenssoziologische Diskursanalyse«, in: Reiner Keller/Andreas Hirseland/Werner Viehöver/Willy Schneider (Hg.), Handbuch Sozialwissenschaftliche Diskursanalyse, Wiesbaden: VS, S. 113-143.

Keller, Reiner (2004): Diskursforschung. Eine Einführung für SozialwissenschaftlerInnen, Opladen: Leske + Budrich.

Keller, Reiner (2009): Müll - Die gesellschaftliche Konstruktion des Wertvollen. Die öffentliche Diskussion über Abfall in Deutschland und Frankreich, Wiesbaden: VS.

Keller, Reiner (2010): »Nach der Gouvernementalitätsforschung und nach dem Poststrukturalismus? Anmerkungen aus der Sicht der wissenssoziologischen Diskursanalyse«, in: Johannes Angermüller/Silke van Dyk (Hg.), Diskursanalyse meets Gouvernementalitätsforschung. Perspektiven auf das Verhältnis von Subjekt, Sprache, Macht und Wissen, Frankfurt a.M.: Campus, S. 43-70.

Keller, Reiner (2011): Diskursforschung. Eine Einführung für SozialwissenschaftlerInnen, Wiesbaden: VS.

Keller, Reiner/Hirseland, Andreas/Viehöver, Werner/Schneider, Willy (Hg.) (2001): Handbuch Sozialwissenschaftliche Diskursanalyse, Wiesbaden: VS.

Keller, Reiner/Hirseland, Andreas/Schneider, Werner/Viehöver Willy (2001): »Zur Aktualität sozialwissenschaftlicher Diskursanalyse - Eine Einführung«, in: Dies.: Handbuch Sozialwissenschaftliche Diskursanalyse, Wiesbaden: VS, S. 7-27. 
Keller, Reiner/Schneider, Werner/Viehöver, Willy (Hg.) (2008): Diskurs, Macht, Subjekt. Wiesbaden: VS.

Kendall, Gavin (2011): »Foucauldian Approaches to the Self«, in: Anthony Elliott (Hg.), Routledge Handbook of identity studies, London: Routledge, S. 67-82.

Kessl, Fabian (2010): »Diskursanalytische Vorgehensweisen«, in: Karin Bock/ Ingrid Miethe/Bettina Ritter (Hg.), Handbuch qualitative Methoden in der sozialen Arbeit, Opladen: Budrich, S. 346-352.

Kessl, Fabian/Krasmann, Susanne (2005): »Sozialpolitische Programmierungen«, in: Fabian Kessl/Christian Reutlinger/Susanne Maurer/Oliver Frey (Hg.), Handbuch Sozialraum, Wiesbaden: VS, S. 227-245.

Kessl, Fabian/Reutlinger, Christian/Maurer, Susanne/Frey, Oliver (Hg.) (2005): Handbuch Sozialraum, Wiesbaden: VS.

Kocyba, Hermann (2009): »Aktivierung«, in: Ulrich Bröckling/Susanne Krasmann/Thomas Lemke (Hg.), Glossar der Gegenwart, Frankfurt a.M.: Suhrkamp, S. 17-22.

Koller, Hans-Christoph (1999): Bildung und Widerstreit. Zur Struktur biographischer Bildungsprozesse in der (Post-)Moderne, München: Fink.

Koloma Beck, Teresa/Schlichte, Klaus (2014): Theorien der Gewalt zur Einführung, Hamburg: Junius.

Kögler, Hans-Herbert (2007): »Die Macht der Interpretation: Kritische Sozialwissenschaft im Anschluss an Foucault«, in: Roland Anhorn/Frank Bettinger/Johannes Stehr (Hg.), Foucaults Machtanalytik und Soziale Arbeit. Eine kritische Einführung und Bestandsaufnahme, Wiesbaden: VS, S. 348-363.

König, Tomke (2008): »Diskurstheorie als Werkzeugkiste: eine Analyse der Effekte diskursiver Praktiken im Kontext familialer Geschlechterarrangements«, in: Karl-Siegbert Rehberg/Dana Giesecke (Hg.), Die Natur der Gesellschaft. Verhandlungen des 33. Kongresses der Deutschen Gesellschaft für Soziologie in Kassel 2006, Frankfurt a.M.: Campus, S. 4787-4797.

Krasmann, Susanne (Hg.) (1997): Die Gewalt in der Kriminologie, Weinheim: Juventa.

Krasmann, Susanne (1997): »Andere Orte der Gewalt«, in: Dies., Die Gewalt in der Kriminologie, Weinheim: Juventa, S. 85-102.

Krasmann, Susanne (2011): »Der Präventionsstaat im Einvernehmen. Wie Sichtbarkeitsregime stillschweigend Akzeptanz produzieren«, in: Leon Hempel/Susanne Krasmann/Ulrich Bröckling (Hg.), Sichtbarkeitsregime. Überwachung, Sicherheit und Privatheit im 21. Jahrhundert, Wiesbaden: VS, S. 53-70.

Krasmann, Susanne/Scheerer, Sebastian (1997): »Die kritische Kriminologie und das Jahrhundert der Gewalt«, in: Susanne Krasmann (Hg.), Die Gewalt in der Kriminologie, Weinheim: Juventa, S. 3-15. 
Kreissl, Reinhard (1997): »Überlegungen zur Kritik des kriminologischen Gewaltdiskurses«, in: Susanne Krasmann (Hg.), Die Gewalt in der Kriminologie, Weinheim: Juventa, S. 183-198.

Kruse, Jan (2011, Oktober): Reader »Einführung in die Qualitative Interviewforschung«, Freiburg: Universität.

Kuhlmann, Carola (2012): »Der Begriff der Inklusion im Armuts- und Menschenrechtsdiskurs der Theorien Sozialer Arbeit - eine historisch-kritische Annäherung«, in: Hans-Jürgen Balz/Benjamin Benz/Carola Kuhlmann (Hg.), Soziale Inklusion. Grundlagen, Strategien und Projekte in der Sozialen Arbeit, Wiesbaden: VS, S. 35-57.

Liell, Christoph (1999): »Der Doppelcharakter von Gewalt: Diskursive Konstruktionen und soziale Praxis«, in: Sighard Neckel/Michael Schwab-Trapp (Hg.), Ordnungen der Gewalt. Beiträge zu einer politischen Soziologie der Gewalt und des Krieges, Opladen: Leske + Budrich, S. 33-54.

Liell, Christoph (2002): »Gewalt in modernen Gesellschaften - zwischen Ausblendung und Dramatisierung «, in: Aus Politik und Zeitgeschichte 44, S. 6-13.

Lindenau, Mathias (2012): »Prävention als Form sybillinischer Weissagung«, in: Mathias Lindenau/Marcel Meier Kressig (Hg.), Zwischen Sicherheitserwartung und Risikoerfahrung. Vom Umgang mit einem gesellschaftlichen Paradoxon in der Sozialen Arbeit, Bielefeld: transcript, S. 325-350.

Lindenau, Mathias/Meier Kressig, Marcel (Hg.) (2012): Zwischen Sicherheitserwartung und Risikoerfahrung. Vom Umgang mit einem gesellschaftlichen Paradoxon in der Sozialen Arbeit, Bielefeld: transcript.

Lindenau, Mathias/Münkler, Herfried (2012): »Vom Orakel zur Risikoanalyse: Figurationen von Sicherheit und Risiko«, in: Matthias Lindenau/Marcel Meier Kressig (Hg.), Zwischen Sicherheitserwartung und Risikoerfahrung. Vom Umgang mit einem gesellschaftlichen Paradoxon in der Sozialen Arbeit, Bielefeld: transcript, S. 21-74.

Lindenberg, Michael/Ziegler, Holger (2005): »Prävention«, in: Fabian Kessl/ Christian Reutlinger/Susanne Maurer/Oliver Frey (Hg.), Handbuch Sozialraum, Wiesbaden: VS, S. 611-627.

Lindner, Werner/Freund, Thomas (Hg.) (2001): Prävention. Zur kritischen Bewertung von Präventionsansätzen in der Jugendarbeit, Opladen: Leske + Budrich.

Lindner, Werner/Freund, Thomas (2001). »Der Prävention vorbeugen? Zur Reflexion und kritischen Bewertung von Präventionsaktivitäten in der Sozialpädagogik«, in: Dies. (Hg.), Prävention. Zur kritischen Bewertung von Präventionsansätzen in der Jugendarbeit, Opladen: Leske + Budrich, S. 69-96. Lüders, Christian/Meuser, Michael (1997): »Deutungsmusteranalyse«, in: Ronald Hitzler/Anne Honer (Hg.), Sozialwissenschaftliche Hermeneutik. Eine Einführung, Opladen: Leske + Budrich, S. 57-79. 
Luedtke, Jens/Wiezorek, Christine (Hg.) (2016): Jugendpolitiken. Wie geht Gesellschaft mit »ihrer« Jugend um?, Weinheim/Basel: Beltz Juventa.

Luedtke, Jens/Wiezorek, Christine (2016): »Jugendpolitiken. Wie geht Gesellschaft mit >ihrer< Jugend um? Eine Einleitung«, in: Dies. (Hg.), Jugendpolitiken. Wie geht Gesellschaft mit »ihrer« Jugend um?, Weinheim/Basel: Beltz Juventa, S. 7-25.

Luutz, Wolfgang (Hg.) (1994): »Das soziale Band ist zerrissen«. Sprachpraktiken sozialer Desintegration, Leipzig: Leipziger Universitätsverlag.

Luutz, Wolfgang (1994): »Diskurse als Schnittstellen zwischen sprachlichem und außersprachlich-sozialem Handeln«, in: Ders. (Hg.), »Das soziale Band ist zerrissen«. Sprachpraktiken sozialer Desintegration, Leipzig: Leipziger Universitätsverlag, S. 43-68.

Mannheim, Karl (1980): Strukturen des Denkens, Frankfurt a.M.: Suhrkamp. Marktschukat, Jürgen (2001): »Diskurse und Gewalt: Wege zu einer Todesstrafe im 18. und 19. Jahrhundert«, in: Reiner Keller/Andreas Hirseland/Werner Viehöver/Willy Schneider (Hg.), Handbuch Sozialwissenschaftliche Diskursanalyse, Wiesbaden: VS, S. 67-95.

Meuser, Michael (1989): Gleichstellung auf dem Prüfstand. Frauenförderung in der Verwaltungspraxis, Pfaffenweiler: Centaurus.

Meuser, Michael/Nagel, Ulrike (2009a): »Experteninterview und der Wandel der Wissensproduktion«, in: Alexander Bogner (Hg.), Experteninterviews. Theorien, Methoden, Anwendungsfelder, Wiesbaden: VS, S. 35-6o.

Meuser, Michael/Nagel, Ulrike (2009b): »ExpertInneninterviews - vielfach erprobt, wenig bedacht«, in: Alexander Bogner (Hg.), Experteninterviews. Theorien, Methoden, Anwendungsfelder, Wiesbaden: VS, S. 71-93.

Meuser, Michael/Nagel, Ulrike (2010): »ExpertInneninterview: Zur Rekonstruktion spezialisierten Sonderwissens«, in: Ruth Becker/Beate Kortendiek (Hg.), Handbuch Frauen- und Geschlechterforschung. Theorie, Methoden, Empirie, Wiesbaden: VS/GWV, S. 376-379.

Meyer, Sebastian (2016): »Der Klient als Risiko. Eine empirische Studie über das Versiegen der Lebensweltorientierung im Allgemeinen Sozialen Dienst«, in: Widersprüche 36 (140), S. 121-133.

Miethe, Ingrid/Müller, Hans-Rüdiger (Hg.) (2012): Qualitative Bildungsforschung und Bildungstheorie, Opladen: Budrich.

Miller, Gale/Holstein, James A. (Hg.) (1993): Constructionist controversies. Issues in social problems theory, New York: Aldine de Gruyter.

Müller, Jan (2013): »>Anerkennenく und >Anrufenく. Figuren der Subjektivierung«, in: Andreas Gelhard/Thomas Alkemeyer/Norbert Ricken (Hg.), Techniken der Subjektivierung, München: Wilhelm Fink, S. 61-78.

Münkler, Herfried (2009): »Einleitung«, in: Herfried Münkler/Jens Hacke (Hg.), Strategien der Visualisierung. Verbildlichung als Mittel politischer Kommunikation, Frankfurt a.M.: Campus, S. 7-9. 
Münkler, Herfried/Hacke, Jens (Hg.) (2009): Strategien der Visualisierung. Verbildlichung als Mittel politischer Kommunikation, Frankfurt a.M.: Campus.

Neckel, Sighard/Schwab-Trapp, Michael (Hg.) (1999): Ordnungen der Gewalt. Beiträge zu einer politischen Soziologie der Gewalt und des Krieges, Opladen: Leske + Budrich.

Neumann-Braun, Klaus/Deppermann, Arnulf (1998): »Ethnographie der Kommunikationskulturen Jugendlicher. $\mathrm{Zu}$ Gegenstandskonzeption und Methodik der Untersuchung von Peer-Groups«, in: Zeitschrift für Soziologie 27 (4), S. 239-255.

Nohl, Arnd-Michael (2006): Interview und dokumentarische Methode, Wiesbaden: VS.

Oelkers, Nina (2013): »Responsibilisierung oder Verantwortungsaktivierung in der Sozialen Arbeit«, in: Nina Oelkers/Martina Richter (Hg.), Aktuelle Themen und Theoriediskurse in der Sozialen Arbeit, Frankfurt a.M.: Peter Lang, S. 163-174.

Oelkers, Nina/Richter, Martina (Hg.) (2013): Aktuelle Themen und Theoriediskurse in der Sozialen Arbeit, Frankfurt a.M.: Peter Lang.

Opitz, Sven (2007): Foucault als Theoretiker der Inklusion und Exklusion, in: Roland Anhorn/Frank Bettinger/Johannes Stehr (Hg.), Foucaults Machtanalytik und Soziale Arbeit. Eine kritische Einführung und Bestandsaufnahme, Wiesbaden: VS, S. 41-58.

Ott, Marion/Wrana, Daniel (2010): »Gouvernementalität diskursiver Praktiken. Zur Methodologie der Analyse von Machtverhältnissen am Beispiel einer Maßnahme zur Aktivierung von Erwerbslosen«, in: Johannes Angermüller/Silke van Dyk (Hg.), Diskursanalyse meets Gouvernementalitätsforschung. Perspektiven auf das Verhältnis von Subjekt, Sprache, Macht und Wissen, Frankfurt a.M.: Campus, S. 155-181.

Patzelt, Werner J. (1987): Grundlagen der Ethnomethodologie: Theorie, Empirie und politikwissenschaftlicher Nutzen einer Soziologie des Alltags, München: Wilhelm Fink.

Popitz, Heinrich (2003): Über die Präventivwirkung des Nichtwissens, Berlin: BWV.

Postman, Neil (1982): The disappearance of childhood, London: W.H. Allen. Raphael, Lutz (1996): »Die Verwissenschaftlichung des Sozialen als methodische und konzeptionelle Herausforderung für eine Sozialgeschichte des 20. Jahrhunderts«, in: Geschichte und Gesellschaft 22 (2), S. 165-193.

Rapetti, Nicoletta (2016): »Ganzheitlichkeit oder Totalität? Absurde Überlegungen zu einer präventiven Sozialen Arbeit im Gesundheitswesen«, in: Widersprüche 36 (139), S. 37-50.

Reckwitz, Andreas (2000): Die Transformation der Kulturtheorien. Zur Entwicklung eines Theorieprogramms, Weilerswist: Velbrück. 
Reckwitz, Andreas (2003): »Grundelemente einer Theorie sozialer Praktiken. Eine sozialtheoretische Perspektive«, in: Zeitschrift für Soziologie 32 (4), S. 282-301.

Reckwitz, Andreas (2008a): Subjekt, Bielefeld: transcript.

Reckwitz, Andreas (2008b): »Praktiken und Diskurse. Eine sozialtheoretische und methodologische Relation«, in: Herbert Kalthoff (Hg.), Theoretische Empirie. Zur Relevanz qualitativer Forschung, Frankfurt a.M.: Suhrkamp, S. 188-209.

Reder, Robin/Ziegler, Holger (2011): »Kriminalprävention und Soziale Arbeit«, in: Bernd Dollinger/Henning Schmidt-Semisch (Hg.), Handbuch Jugendkriminalität. Kriminologie und Sozialpädagogik im Dialog, Wiesbaden: VS, S. 365-377.

Reh, Sabine (2003): Berufsbiographische Texte ostdeutscher Lehrer und Lehrerinnen als »Bekenntnisse «. Interpretationen und methodologische Überlegungen zur erziehungswissenschaftlichen Biographieforschung, Bad Heilbrunn/Obb.: Klinkhardt.

Reh, Sabine/Ricken, Norbert (2012): »Das Konzept der Adressierung. Zur Methodologie einer qualitativ-empirischen Erforschung von Subjektivation«, in: Ingrid Miethe/Hans-Rüdiger Müller (Hg.), Qualitative Bildungsforschung und Bildungstheorie, Opladen: Budrich, S. 35-56.

Rehberg, Karl-Siegbert/Giesecke, Dana (Hg.) (2016): Die Natur der Gesellschaft. Verhandlungen des 33. Kongresses der Deutschen Gesellschaft für Soziologie in Kassel 2006, Frankfurt a.M.: Campus.

Riegel, Christine/Scherr, Albert/Stauber, Barbara (Hg.) (2010): Transdisziplinäre Jugendforschung. Grundlagen und Forschungskonzepte, Wiesbaden: VS.

Riegraf, Birgit/Spreen, Dierk/Mehlmann, Sabine (Hg.) (2012): Medien - Körper - Geschlecht. Diskursivierungen von Materialität; Festschrift für Hannelore Bublitz. Unter Mitarbeit von Hannelore Bublitz, Bielefeld: transcript.

Sack, Fritz (1995): »Prävention - Ein alter Gedanke in einem neuen Gewand. Zur Entwicklung und Kritik der Strukturen >postmoderner Kontrolle<, in: Rolf Gössner (Hg.), Mythos Sicherheit. Der hilflose Schrei nach dem starken Staat, Baden-Baden: Nomos, S. 429-456.

Sack, Fritz (2002): »Einführende Anmerkungen zur Kritischen Kriminologie«, in: Roland Anhorn (Hg.), Kritische Kriminologie und soziale Arbeit. Impulse für professionelles Selbstverständnis und kritisch-reflexive Handlungskompetenz, Weinheim: Juventa, S. 27-45.

Sandermann, Philipp (2013): »Funktion und Stellenwert von Kritik in Beiträgen zur Sozialen Arbeit und ihr Verhältnis zu Normativität - eine Positionierung«, in: Benjamin Benz/Günter Rieger/Werner Schönig/Monika Többe-Schukalla (Hg.), Politik Sozialer Arbeit. Band 1: Grundlagen, theoretische Perspektiven und Diskurse, Weinheim: Beltz Juventa, S. 287-298. 
Scherr, Albert (2010): »Für eine strukturtheoretisch fundierte kritisch-reflexive Jugendforschung - Konturen einer transdisziplinären Perspektive«, in: Christine Riegel/Albert Scherr/Barbara Stauber (Hg.), Transdisziplinäre Jugendforschung. Grundlagen und Forschungskonzepte, Wiesbaden: VS, S. $47-63$.

Scherr, Albert (2014): »Jugend als soziale Kategorie. Oder: Warum Jugend keine Gruppe und auch kein soziales Problem ist«, in: Axel Groenemeyer/ Dagmar Hoffmann (Hg.), Jugend als soziales Problem - soziale Probleme der Jugend? Diagnosen, Diskurse und Herausforderungen, Weinheim: Beltz-Juventa, S. 29-49.

Schierz, Sascha (2013): »Soziale Kontrolle. Prävention und soziale Probleme als Denkfolien Sozialer Arbeit und sozialpädagogischen Handelns«, in: Nina Oelkers/Martina Richter (Hg.), Aktuelle Themen und Theoriediskurse in der Sozialen Arbeit, Frankfurt a.M.: Peter Lang, S. 29-44.

Schmidt, Friederike (2015): »Konstruktionen von Devianz im Blick pädagogischer Fachpersonen«, in: Bernd Dollinger/Nina Oelkers (Hg.), Sozialpädagogische Perspektiven auf Devianz, Weinheim: Beltz Juventa, S. 102-117.

Schönig, Werner (2013): »Soziale Arbeit als Intervention und Modus der Sozialpolitik«, in: Benjamin Benz/Günter Rieger/Werner Schönig/Monika Többe-Schukalla (Hg.), Politik Sozialer Arbeit. Band 1: Grundlagen, theoretische Perspektiven und Diskurse, Weinheim: Beltz Juventa, S. 32-53.

Schrage, Dominik (2012): »Subjektivierung durch Normalisierung«, in: Birgit Riegraf/Dierk Spreen/Sabine Mehlmann (Hg.), Medien - Körper - Geschlecht. Diskursivierungen von Materialität; Festschrift für Hannelore Bublitz. Unter Mitarbeit von Hannelore Bublitz, Bielefeld: transcript, S. 7388.

Schreiber, Verena (2011): Fraktale Sicherheiten. Eine Kritik der kommunalen Kriminalprävention, Bielefeld: transcript.

Schulz, Christa/Wambach, Manfred Max (1983): »Vorbemerkungen. Oberfläche und Dunkelfeld«, in: Manfred Max Wambach (Hg.), Der Mensch als Risiko. Zur Logik von Prävention und Früherkennung, Frankfurt a.M.: Suhrkamp, S. 7-10.

Schröder, Peter/Steger, Hugo (Hg.) (1981): Dialogforschung. Jahrbuch 1980 des Instituts für deutsche Sprache, Düsseldorf: Pädagogischer Verlag Schwann. Schwab-Trapp, Michael (2001): »Methodische Aspekte der Diskursanalyse. Probleme der Analyse diskursiver Auseinandersetzungen am Beispiel der deutschen Diskussion über den Kosovokrieg«, in: Reiner Keller/Andreas Hirseland/Werner Viehöver/Willy Schneider (Hg.), Handbuch Sozialwissenschaftliche Diskursanalyse, Wiesbaden: VS, S. 169-195.

Smaus, Gerlinda (1986): »Versuch um eine materialistisch-interaktionistische Kriminologie«, in: Arbeitskreis junger Kriminologen (Hg.), Kritische Kri- 
minologie heute. 1. Beiheft des Kriminologischen Journals, Weinheim: Juventa, S. 179-199.

Spector, Malcolm/Kitsuse, John I. (1977): Constructing Social Problems, Menlo Park: Cummings.

Staudigl, Michael (2007): »Towards a Phenomenological Theory of Violence: Reflections Following Mereau-Ponty and Schutz«, in: Hum Stud 30, S. 233253.

Stangl, Wolfgang (1986): »Staatliche Normgenese und symbolischer Interaktionismus «, in: Arbeitskreis junger Kriminologen (Hg.), Kritische Kriminologie heute. 1. Beiheft des Kriminologischen Journals, Weinheim: Juventa, S. 121-132.

Stehr, Johannes (2009): »Jugendgewalt - Skandalisierungskonzept und ideologische Kategorie«, in: Otger Autrata/Bringfriede Scheu (Hg.), Jugendgewalt. Interdisziplinäre Sichtweisen, Wiesbaden: VS, S. 107-124.

Steiner, Olivier (2002): »Gesellschaft, Identität und Jugendgewalt«, in: Matthias Drilling (Hg.), Gewalt an Schulen: Ursachen, Prävention, Intervention. Beiträge der 2. Nationalen Fachtagung der Stiftung Erziehung zur Toleranz, Zürich: Pestalozzianum, S. 14-24.

Steinert, Heinz (1995): »Prävention als kommunale Aufgabe. Jenseits von Polizei und Strafrecht«, in: Rolf Gössner (Hg.), Mythos Sicherheit. Der hilflose Schrei nach dem starken Staat, Baden-Baden: Nomos, S. 403-414.

Strauß, Sarah (2012): Peer Education \& Gewaltprävention. Theorie und Praxis dargestellt am Projekt Schlag.fertig, Freiburg i.B.: Centaurus.

Strauss, Anselm L./Corbin, Juliet M./Niewiarra, Solveigh (Hg.) (1996): Grounded Theory: Grundlagen qualitativer Sozialforschung, Weinheim: Beltz.

Tenbruck, Friedrich H. (1965): Jugend und Gesellschaft. Soziologische Perspektiven, Freiburg i.B.: Rombach.

Topitsch, Ernst (Hg.) (1960): Probleme der Wissenschaftstheorie. Festschrift für Victor Kraft. Unter Mitarbeit von Victor Kraft, Wien: Springer.

Topitsch, Ernst (1960): »Über Leerformeln. Zur Pragmatik des Sprachgebrauchs in Philosophie und politischer Theorie«, in: Ders. (Hg.), Probleme der Wissenschaftstheorie. Festschrift für Victor Kraft. Unter Mitarbeit von Victor Kraft, Wien: Springer, S. 233-264.

Trotha, Trutz von (Hg.) (1997): Soziologie der Gewalt, Opladen: Westdeutscher Verlag.

Trotha, Trutz von (1997): »Zur Soziologie der Gewalt«, in: Ders. (Hg.), Soziologie der Gewalt, Opladen: Westdeutscher Verlag, S. 9-56.

Turner, G./Shepherd, J. (1999): »A method in search of a theory: peer education and health promotion«, in: Health Education Research 14 (2), S. 235-247.

Vogelmann, Frieder (2013): »Verantwortung als Subjektivierung. Zur Genealogie einer Selbstverständlichkeit«, in: Andreas Gelhard/Thomas Alkemey- 
er/Norbert Ricken (Hg.), Techniken der Subjektivierung, München: Wilhelm Fink, S. 149-161.

Vogelmann, Frieder (2014): Im Bann der Verantwortung, Frankfurt a.M.: Campus.

Wambach, Manfred Max (Hg.) (1983): Der Mensch als Risiko. Zur Logik von Prävention und Früherkennung, Frankfurt a.M.: Suhrkamp.

Wedl, Juliette/Wrana, Daniel (2014): »Einleitung. Grundfragen der Forschungspraxis«, in: Johannes Angermüller/Martin Nonhoff/Eva Herschinger (Hg.), Diskursforschung. Ein interdisziplinäres Handbuch, Bielefeld: transcript, S. 479-481.

Weyers, Stefan (2006): »Verantwortung/Eigenverantwortung«, in: Bernd Dollinger/Jürgen Raithel (Hg.): Aktivierende Sozialpädagogik. Ein kritisches Glossar, Wiesbaden: VS, S. 217-233.

Widersprüche. Zeitschrift für sozialistische Politik im Bildungs-, Gesundheits- und Sozialbereich (2001): Alles im Griff. Prävention als Sozialtechnologie, Heft 79.

Widersprüche. Zeitschrift für sozialistische Politik im Bildungs-, Gesundheits- und Sozialbereich (2016): Politik der Prävention. unvorsichtig - riskant - widersprüchlich, Heft 139.

Wiedemann, Peter Michael (1989): »Deutungsmusteranalyse«, in: Gerd Jüttemann (Hg.), Qualitative Forschung in der Psychologie, Heidelberg: Roland Asanger, S. 212-226.

Wrana, Daniel (2012): »Theoretische und methodologische Grundlagen der Analyse diskursiver Praktiken«, in: Daniel Wrana/Christiane Maier Reinhard (Hg.), Professionalisierung in Lernberatungsgesprächen. Theoretische Grundlegungen und empirische Untersuchungen, Opladen: Barbara Budrich, S. 195-214.

Wrana, Daniel/Maier Reinhard, Christiane (Hg.) (2012): Professionalisierung in Lernberatungsgesprächen. Theoretische Grundlegungen und empirische Untersuchungen, Opladen: Barbara Budrich.

Ziegler, Holger (2001): »Community Diskurse in Jugendhilfe und Kriminalprävention - Der Tod und die lokale Wiederauferstehung des Sozialen in der Kontrolle«, in: Soziale Probleme. Zeitschrift für soziale Probleme und soziale Kontrolle. Konstruktivistische Kontroversen und gesellschaftliche Herausforderungen 12 (1/2), S. 183-207. 


\section{Soziologie}

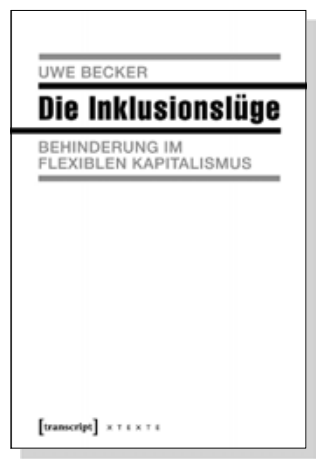

Uwe Becker

Die Inklusionslüge

Behinderung im flexiblen Kapitalismus

2015, 216 S., kart., 19,99 $€(D E)$,

ISBN 978-3-8376-3056-5

E-Book: $17,99 €(\mathrm{DE})$, ISBN $978-3-8394-3056-9$

EPUB: $17,99 €(\mathrm{DE})$, ISBN 978-3-7328-3056-5

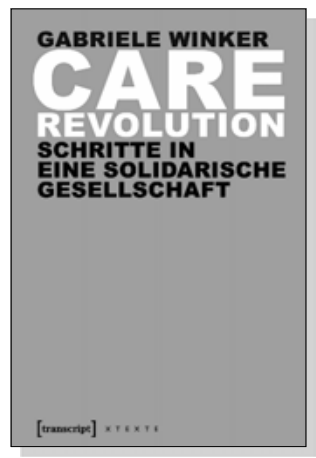

Gabriele Winker

Care Revolution

Schritte in eine solidarische Gesellschaft

2015, 208 S., kart., 11,99€ (DE),

ISBN 978-3-8376-3040-4

E-Book: $10,99 €(D E)$, ISBN 978-3-8394-3040-8

EPUB: $10,99 €(D E)$, ISBN 978-3-7328-3040-4

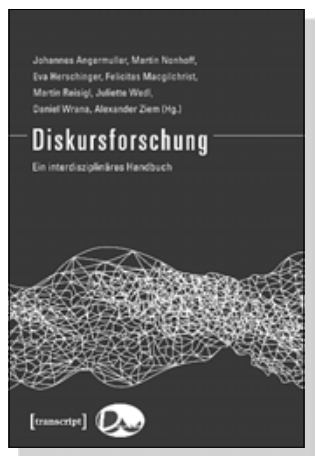

Johannes Angermuller, Martin Nonhoff, Eva Herschinger, Felicitas Macgilchrist, Martin Reisigl, Juliette Wedl, Daniel Wrana, Alexander Ziem (Hg.)

\section{Diskursforschung}

Ein interdisziplinäres Handbuch (2 Bde.)

2014, 1264 S., kart., 2 Bde. im Schuber, zahlr. Abb. $44,99 €(\mathrm{DE})$, ISBN 978-3-8376-2722-0

E-Book: $44,99 €(\mathrm{DE})$, ISBN 978-3-8394-2722-4 


\section{Soziologie}

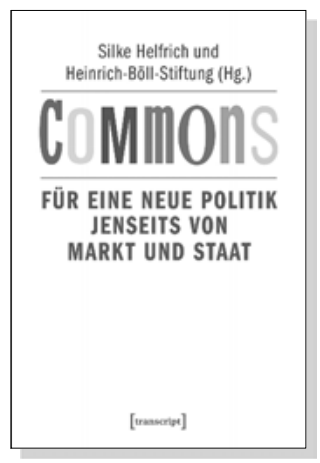

Silke Helfrich, Heinrich-Böll-Stiftung (Hg.)

\section{Commons}

Für eine neue Politik

jenseits von Markt und Staat

2014, 528 S., kart., $24,80 €(D E)$,

ISBN 978-3-8376-2835-7

als Open-Access-Publikation kostenlos erhältlich

E-Book: ISBN 978-3-8394-2835-1

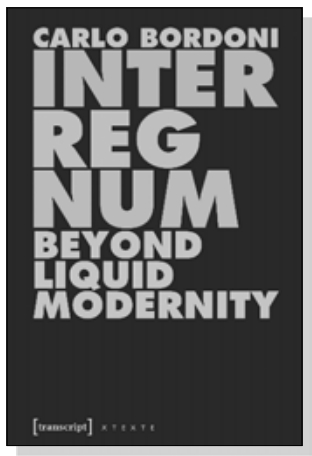

Carlo Bordoni

\section{Interregnum}

Beyond Liquid Modernity

März 2016, 136 p., 19,99€ (DE),

ISBN 978-3-8376-3515-7

E-Book: $17,99 €(\mathrm{DE})$, ISBN $978-3-8394-3515-1$

EPUB: $17,99 €(D E)$, ISBN 978-3-7328-3515-7

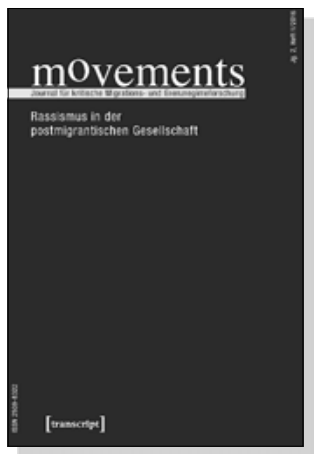

Kijan Espahangizi, Sabine Hess, Juliane Karakayali, Bernd Kasparek, Simona Pagano, Mathias Rodatz, Vassilis S. Tsianos (Hg.) movements. Journal für kritische Migrationsund Grenzregimeforschung

Jg. 2, Heft 1/2016:

Rassismus in der postmigrantischen Gesellschaft

September 2016, 272 S., kart.

24,99€ (DE), ISBN 978-3-8376-3570-6

als Open-Access-Publikation kostenlos erhältlich:

www.movements-journal.org 